\title{
Laboruntersuchungen zum langfristigen \\ Denitrifikationspotential im oberflächennahen \\ Grundwasser hydromorpher Mineralböden \\ Nordwestdeutschlands
}

\author{
DISSERTATION \\ Zur Erlangung des Doktorgrades \\ doctora scientiarum agrariarum \\ Dr. sc. agr. \\ der Fakultät für Agrarwissenschaften \\ der Georg-August-Universität Göttingen
}

Vorgelegt von

Ozra Mehranfar

Dipl. Ing. Agr.

Göttingen 2003 
Gutachter: $\quad$ Prof. Dr. B. Meyer, Universität Göttingen Prof. Dr. N. Classen

Tag der mündlichen Prüfung: 11.07.03 
Diese Arbeit wurde mit Mitteln der Deutschen BundesstifTung UMWELT gefördert. 


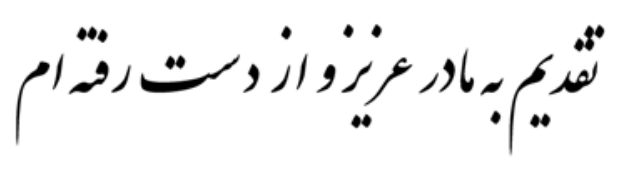

Meiner lieben Mutter 


\section{Abstract}

In the shallow groundwater of hydromorphic soils denitrification plays an important role for reducing nitrate and production of nitrous oxide. Depending on the presence of fossil or recent reductants potential rates of denitrification can vary considerably. There is concern that permanent nitrate leaching caused by agricultural management may deplete the stocks of available reductants (organic carbon, sulfides). On a long-term basis this would cause decreasing rates of nitrate reduction and thus increasing nitrate levels in groundwater. Until now, there has been a lack of knowledge concerning the magnitude of the total stocks and the microbial availability of organic carbon and sulfides in the shallow groundwater of hydromorphic soils

The objective of this study was to describe long-term denitrification dynamics in the saturated zone of hydromorphic soils under agricultural management. This included (i) quantification of the total stocks of reduced compounds, (ii) measuring the time course of nitrate reduction during proceeding exhaustion of available reductants, and (iii) modeling long-term denitrification potential of hydromorphic soils using soil parameters which are easy to determine.

The research program included 11 sites located in Lower Saxony, Germany. The soils were gleyic Podzols, gleyic and stagnogleyic Fluvisols or Anthroposols. Sites represented a broad range of properties (soil type, stock of reductants, textures, groundwater level) in order to represent hydromorphic mineral soils under agricultural management in Lower Saxony as good as possible.

Soil samples were collected from the capillary fringe and close below the groundwater surface between $1 \mathrm{~m}$ (minimum) and $4 \mathrm{~m}$ (maximum) below the soil surface.

Measured parameters were texture, $\mathrm{pH}$, carbonate, organic carbon (total, soluble and hotwater soluble) and sulfur (total, sulfide, sulfate).

Long-term denitrification was measured by long-term incubation of soil samples under denitrifying conditions. Soil slurries were amended with $\mathrm{K}^{15} \mathrm{NO}_{3}$ solution and were incubated at $25^{\circ} \mathrm{C}$ anaerobically for up to 700 days. To determine temperature dependence of denitrification some of the samples were additionally incubated at $10^{\circ} \mathrm{C}$. To determine the time course of denitrification and sulfate production the bottles were sampled repeatedly during the incubation and samples were analyzed for dissolved nitrate, sulfate and 
${ }^{15}\left(\mathrm{~N}_{2}+\mathrm{N}_{2} \mathrm{O}\right)$ in the gas phase. In addition to that, parallels of the samples were incubated aerobically in order to determine oxidizable sulfide.

Stocks of organic carbon and sulfide varied considerably within and between sites. Vertical distribution showed in some cases concentrations decreasing with depth, whereas other sites exhibited maximum values close below the groundwater surface. Some of the gleyic Podzols and the stagnogleyic Fluvisol did not contain any sulfides at all and contained low levels of $\mathrm{C}_{\text {org }}(<0,2 \%)$. Other gleyic Podzols had $\mathrm{C}_{\text {org }}$-contents up to $1 \%$ and contained low sulfide contents in the deeper samples. The marine gleyic Fluvisol had a medium $\mathrm{C}_{\text {org }}$ level (up to 1 $\mathrm{mg} \mathrm{kg}^{-1}$ ) but a relatively high sulfide content (up to $1 \mathrm{mg} \mathrm{S} \mathrm{kg-1).} \mathrm{The} \mathrm{riverine} \mathrm{gleyic}$ Fluvisols consistently exhibited high $\mathrm{C}_{\text {org }}$ (up to $10 \%$ ) and sulfide contents (up to $8 \mathrm{mg} \mathrm{kg}^{-1}$ ).

During anaerobic incubation rates of nitrate reduction $\left(\mathrm{R}_{\mathrm{NO} 3}\right)$ decreased in all of the samples. In the gleyic Podzols with low $\mathrm{C}_{\text {org }}$ levels rates were initially lowest $\left(<0,2 \mathrm{mg} \mathrm{N} \mathrm{kg}^{-1} \mathrm{~d}^{-1}\right)$ and dropped to zero before the end of the incubation.

In the soils with high stocks of reductants $\mathrm{R}_{\mathrm{NO} 3}$ was initially up to $2,3 \mathrm{mg} \mathrm{N} \mathrm{kg}^{-1} \mathrm{~d}^{-1}$ and dropped to approx. 1/3 of initial activity. Autotrophic denitrification was proved by sulfate production in all samples containing sulfides. Sulfate production rates decreased faster than nitrate reduction rates.

Long-term potentials of total denitrification $\left(\mathrm{M}_{\mathrm{NO} 3}\right)$ and of autotrophic denitrification $\left(\mathrm{M}_{\mathrm{SO} 4}\right)$ were calculated by integrating the time curves of nitrate reduction and sulfate production. Site averages of $\mathrm{M}_{\mathrm{NO} 3}$ ranged between 10 and $38 \mathrm{mg} \mathrm{N} \mathrm{kg}^{-1}$ in the soils containing no sulfides and low levels of $\mathrm{C}_{\text {org }}$, between 620 and $1100 \mathrm{mg} \mathrm{N} \mathrm{kg}^{-1}$ in the gleyic Fluvisols and between 56 and $132 \mathrm{mg} \mathrm{N} \mathrm{kg}^{-1}$ in the other soils. In the soils containing sulfides, autotrophic denitrification accounted for 12 to $32 \%$ of total long-term denitrification potential.

Availabilities of reductants for denitrification were derived from the ratio of " $\mathrm{M}_{\mathrm{NO} 3} /$ total reductants" or from the ratio "M $\mathrm{MS}_{\mathrm{SO}}$ / sulfide". In most of the soils sulfides were available to a large extent (ca. $10-50 \%$ ) whereas $\mathrm{C}_{\text {org }}$ was only available to a small extent (ca. 2 - $7 \%$ ). Availability of sulfides was higher under aerobic compared to anaerobic conditions.

$\mathrm{M}_{\mathrm{SO} 4}, \mathrm{M}_{\mathrm{NO} 3}, \mathrm{R}_{\mathrm{NO} 3}$ and sulfide availability were significantly correlated with most parameters, where $\mathrm{R}^{2}$ was highest for the relationships with $\mathrm{C}_{\text {org }}$ and sulfide. However, availability of (Sulfid $+\mathrm{C}_{\text {org }}$ ) was not significantly correlated with soil properties.

Statistical models for predicting process rates were developed using multiple regression analysis. The comparison of measured and modeled quantities showed that prediction for individual samples was unsatisfactory. However, the comparison of site averages resulted in a good fit of the model data for $\mathrm{M}_{\mathrm{SO} 4}, \mathrm{M}_{\mathrm{NO} 3}, \mathrm{R}_{\mathrm{NO} 3}$ and sulfide availability. 
To predict long-term groundwater quality in the investigated soils a simple transport and process model was used. Simulations showed that complete exhaustion of denitrification potential in the various sites can be expected in periods between $<10$ years (soils with lowest supply of reductants) to $>200$ years (gleyic Fluvisols with highest supply of reductants).

In this study, exhaustion of denitrification potential resulting from continuous nitrate input into the saturated zone of hydromorphic soils was proved and quantified on a relatively large data set. With respect to long-term groundwater quality it can be concluded that ongoing nitrate input will result in increasing nitrate concentrations in the shallow groundwater of similar sites. In many of the gleyic Fluvisols this might possibly have a significant effect only after several decades from now. For the gleyic Podzols of the glacifluvial sediments it can however be assumed that nitrate reduction rates have already considerably decreased during the preceding decades and that this exhaustion process is still going on. In some cases, exhaustion may already be near completion.

The residual supplies of reductants of hydromorphic mineral soils under agricultural management are important for groundwater protection and must therefore be protected from further exhaustion.

This study proposes various models for predicting long-term denitrification potential. These models can be used to map long-term vulnerability of groundwater resources in lowlands under agricultural management. 


\section{INHALTSVERZEICHNIS}

2.1 Denitrifikationsprozesse in wasserungesättigten Böden 4

2.2 Bedeutung der Denitrifikation 5

2.3 Ökologische Voraussetzungen für die Denitrifikation 7

$\begin{array}{lll}\text { 2.3.1 } & \text { Einfluss von Reduktionsmitteln } & 7\end{array}$

2.3.2 Einfluss der Nitratkonzentration $\quad 8$

2.3.3 Vorhandensein denitrifizierender Mikroorganismen 9

2.3.4 Einfluss des $\mathrm{O}_{2}$-Partialdruckes bzw. Wassergehaltes im Boden 10

$\begin{array}{ll}\text { 2.3.5 Einfluss des pH-Wertes } & 10\end{array}$

2.3.6 Einfluss der Temperatur 11

2.4 Prozesse des Nitratabbaus in hydromorphen Böden 11

2.5 Quellen und Verfügbarkeit von Reduktionsmitteln 13

2.6 Erschöpfbarkeit von Reduktionsmitteln durch Denitrifizierung von Nitrateinträgen

2.7 Auswirkung der Erschöpfung des Reduktionsmittelvorrats auf die Lachgasbildung der Denitrifikation

$3 \quad$ Zielsetzung

$4 \quad$ Material und Methoden $\quad 17$

$\begin{array}{lll}4.1 & \text { Untersuchungsgebiete } & 17\end{array}$

4.2 Probenahme und Probenaufbereitung 20

4.3 Laborexperimente 21

4.3.1 Anaerobe Langzeitinkubationen zur Erschöpfung des Denitrifikations$\begin{array}{ll}\text { potential } & 21\end{array}$

4.3.2 Sulfat-Produktion 23

4.3.3 Aerobe Inkubation zur Bestimmung der mikrobiellen Oxidation des Sulfids23

$\begin{array}{lll}4.4 & \text { Analytik } & 23\end{array}$

$\begin{array}{lll}\text { 4.4.1 Stickstoff } & 23\end{array}$

$\begin{array}{lll}\text { 4.4.2 Kohlenstoff } & 24\end{array}$

4.4.2.1 Gesamt-Kohlenstoff $\left(C_{t}\right)$ und Gesamt-Stickstoff $\left(N_{t}\right) \quad 24$

4.4.2.2 Gesamtorganischer Kohlenstoff $\left(\mathrm{C}_{\text {org }}\right) \quad 25$

4.4.2.3 Gelöster organischer Kohlenstoff $\left(C_{\text {lös }}\right) \quad 25$

4.4.2.4 Heißwasserlöslicher Kohlenstoff $\left(C_{h w l}\right) \quad 25$

4.4.2.5 Carbonat-Kohlenstoff 26

$\begin{array}{lll}4.4 .3 & \text { Schwefel } & 26\end{array}$

4.4.3.1 Gesamt- und Sulfid-Schwefel 26

$\begin{array}{ll}\text { 4.4.3.2 Extrahierbarer Sulfat-Schwefel } & 27\end{array}$

$\begin{array}{lll}\text { 4.4.4 Gelöstes Eisen ( } \mathrm{Fe}_{\text {lös }} \text { ) } & 27\end{array}$ 
4.4.5 Bodenwassergehalt 28

4.4.6 Korngrössenanalyse 28

$\begin{array}{lll}4.4 .7 & \text { pH-Wert } & 29\end{array}$

4.4.8 Gaschromatographie 29

$\begin{array}{lll}\text { 4.4.8.1 Massenspektrometrie } & 30\end{array}$

$5 \quad$ Ergebnisse $\quad 31$

5.1 Chemische Eigenschaften und Korngrößenzusammnesetzung der Böden 31

$\begin{array}{lll}5.2 & \text { Messergebnisse der anaeroben Inkubationsversuche } & 37\end{array}$

5.3 Messergebnisse der aeroben Inkubationsversuche 46

$5.4 \quad$ Einfluss der Temperatur auf die Nitratreduktion und Sulfatproduktion 50

$5.5 \quad$ Flächenmittelwerte der verschiedenen Aktivitäts-Kenngrößen 53

$\begin{array}{lll}6 & \text { Statistische Auswertung } & 58\end{array}$

$\begin{array}{lll}6.1 & \text { Test auf Normalverteilung } & 58\end{array}$

6.2 Korrelationsanalyse 59

$\begin{array}{lll}6.3 & \text { Regressionsanalyse } & 64\end{array}$

6.3.1 Vergleich zwischen gemessenen und berechneten Werten 72

$\begin{array}{lll}7 & \text { Diskussion } & 77\end{array}$

7.1 Variabilität der unabhängigen Parameter und ihre Bedeutung für die $\begin{array}{ll}\text { Denitrifikation } & 77\end{array}$

$\begin{array}{lll}\text { 7.1.1 Gesamt-Vorräte an } \text { Corg }_{\text {org }} \text { und Sulfid } & 77\end{array}$

$\begin{array}{lll}\text { 7.1.2 } & \text { Vorräte an löslichem Kohlenstoff } & 78\end{array}$

$\begin{array}{lll}\text { 7.1.3 C-Verfügbarkeit } & 80\end{array}$

$\begin{array}{lll}\text { 7.1.4 Gehalte an } \mathrm{Fe}^{2+} & 81\end{array}$

$\begin{array}{lll}\text { 7.1.5 } & \text { pH-Wert } & 82\end{array}$

7.2 Denitrifikationsraten, langfristiges Denitrifikationspotential $\quad 82$

7.3 Bedeutung der autotrophen Denitrifikation, Verfügbarkeit von $\mathbf{C}_{\text {org }}$ und

$\begin{array}{lll} & \text { Sulfid für die Denitrifikation } & 83 \\ \text { 7.3.1 } & \text { Autotrophe Denitrifikation } & 83\end{array}$

$\begin{array}{lll}\text { 7.3.2 } & \text { Vergleich von C- und S-Verfügbarkeit } & 84\end{array}$

$\begin{array}{lll}\text { 7.3.3 } & \text { Aerobe Sulfatproduktion } & 85\end{array}$

$7.4 \quad$ Bedeutung der langfristigen Denitrifikationsdynamik für die
Grundwasserqualität

$8 \quad$ Schlussfolgerung und Ausblick $\quad 95$

$\begin{array}{lll}9 & \text { Zusammenfassung } & 98\end{array}$

$\begin{array}{lll}10 & \text { Literatur } & 102\end{array}$

11 Anhang $\quad 116$ 


\section{Verzeichnis der Abbildungen}

Abb. 1

Schritte der Nitratreduktion zu N $\mathrm{N}_{2}$, beteiligte Substrate und Produkte (nach BECKER et al., 1992)

Abb. 2

Der landwirtschaftliche N-Kreislauf (verändert nach S. L. JANSSON, in SCHEFFER \& SCHACHTSCHABEL, 2002)

Abb. 3

Karte der Grundwasserflurabstände in Niedersachsen mit der Lage der untersuchten Böden (nach WENDLAND et al., 1993)

Abb. 4

Tiefenverteilung der Reduktionsmittelvorräte an den untersuchten Standorten

Abb. 5

Anaerobe Inkubation: Zeitverläufe für Nitratreduktion $\left(\mathrm{mg} \mathrm{N} \mathrm{kg}^{-1} \mathrm{~d}^{-1}\right)$, Sulfatproduktion ( $\left.\mathrm{mg} \mathrm{S} \mathrm{kg}^{-1} \mathrm{~d}^{-1}\right), \mathrm{CO}_{2}$-Produktion $\left(\mathrm{mg} \mathrm{C} \mathrm{kg}^{-1} \mathrm{~d}^{-1}\right)$ und ${ }^{15}\left(\mathrm{~N}_{2}+\mathrm{N}_{2} \mathrm{O}\right)$ -

Produktion ( $\mathrm{mg} \mathrm{N} \mathrm{kg}^{-1} \mathrm{~d}^{-1}$ ). Messpunkte gelten jeweils für das vorangegangene Zeitintervall

Abb. 6

Zeitverläufe der anaeroben und aeroben Sulfatproduktion. Messpunkte gelten jeweils für das vorangegangene Zeitintervall

Abb. 7

Zeitverläufe der unterschiedlichen Temperaturen $\left(10^{\circ} \mathrm{C}, 25^{\circ} \mathrm{C}\right)$ für die Nitratreduktion und Sulfatproduktion

Abb. 8

Vergleich zwischen gemessenen und berechneten Werten für die Variablen Nitratreduktionsrate zu Versuchsbeginn (RNO3 (a-c)) und kumulierte Nitratreduktion (MNO3) $(\mathrm{d}-\mathrm{f}))$

Abb. 9

Vergleich eines realen Fließfeldes im oberflächennahen Grundwasser (V = Fließgeschwindigkeit, $\mathrm{dX}=$ Fließstrecke) mit der vereinfachten Annahme einer ausschließlich vertikalen Fließrichtung ( $\mathrm{Vv}, \mathrm{dZ}=$ Vertikalkomponenten der Fließgeschwindigkeit und der Fließstrecke) in einer reaktiven Schicht (gestrichelte Fläche). Die Länge der Pfeile repräsentiert die Beträge der Fließgeschwindigkeiten und der Fließstrecken. Die Verweilzeit in der reaktiven Schicht ergibt sich aus dem Verhältnis von Fließstrecke zur Fließgeschwindigkeit und ist somit für beide Betrachtungsweisen gleich 
Abb. 10

Simulation der Erschöpfung des langfristigen Denitrifikationspotentials $\left(D_{\text {pot }}\right)$ durch fortschreitenden Abbau des Reduktionsmittelvorrats (Beispiel Freistatt, Szenario 1, vgl. Tab. 12). $\mathrm{C}_{\mathrm{NO} 3}$, Rest $=$ Nitratkonzentration im Grundwasser nach Passage der reaktiven Schicht; $\mathrm{DR}=$ Denitrifikationsrate, $\mathrm{T}_{\text {Abbau }}=$ Zeit bis zum vollständigen Abbau des Reduktionsmittelvorrats

Abb. 11

Nitratgehalte des Bodenwassers $\left(\mathrm{C}_{\mathrm{NO} 3}\right)$ bei der Probenahme im Feld im Vergleich zur mittleren Nitratabbaurate $\left(\mathrm{R}_{\mathrm{NO} \text { pot }}, \mathrm{M}\right)$ für die Einzelproben der Böden unter Akkernutzung 


\section{Verzeichnis der Tabellen}

Tab. 1

Vergleich experimentell ermittelter und berechneter Redoxpotentialle für verschiedene Redoxreaktionen bei pH 7 (nach SCHEFFER \& SCHACHTSCHABEL, 1992 in ROLLAND, 1995)

Tab. 2

Untersuchungsgebiete

Tab. 3

Zeitintervalle der anaeroben Inkubation

Tab. 4

Zeitintervalle der aeroben Inkubation

Tab. 5

Schichtmittelwerte der bodenchemischen Parameter sowie der Korngrößenzusammensetzung der untersuchten Lehm-Böden

Tab. 6

Schichtmittelwerte der bodenchemischen Parameter sowie der Korngrößenzusammensetzung der untersuchten Sand-Böden

Tab. 7

Temperatureinfluss auf die Nitratreduktion und die Sulfatproduktion: Verhältnis der Temperaturstufen $\left(25^{\circ} \mathrm{C} / 10^{\circ} \mathrm{C}\right)$

Tab. 8

Zusammenfassung der Ergebnisse zur Reduktion von Nitrat und Produktion von Sulfat aus den anaeroben und aeroben Inkubationsversuchen: Flächenmittelwerte der Umsatzraten zu Versuchsbeginn $\left(\mathrm{R}_{\mathrm{NO} 3}\right.$, DR $\left.{ }^{15}(\mathrm{~N} 2+\mathrm{N} 2 \mathrm{O}), \mathrm{R}_{\mathrm{SO} 4}\right)$, kumulierte Umsetzungen $\left(\mathrm{M}_{\mathrm{NO} 3}, \mathrm{M}_{\mathrm{SO} 4}\right)$, Anteil der heterotrophen Denitrifikation am Gesamtumsatz $\left(\mathrm{M}_{\mathrm{NO} 3} \mathrm{H}: \mathrm{M}_{\mathrm{NO} 3(\mathrm{ges})}\right)$, Nachhaltigkeits- und Verfügbarkeits-Indizies (NI, VI)

Tab. 9a

SPEARMAN-Rang Korrelationskoeffizienten der unabhängigen Bodenparameter

Tab. 9b

SPEARMAN-Rang Korrelationskoeffizienten der abhängigen Bodenparameter

Tab. 10a

Ergebnisse der linearen multiplen Regressionsanlyse zwischen unabhängigen und abhängigen Variablen, Koeffizienten der Regressionsgeraden mit statistischen Kenngrößen. Regressionsverfahren $=$ schrittweise. Auswahl: Alle Porben 
Tab. 10b

Ergebnisse der linearen multiplen Regressionsanalyse zwischen unabhängigen und abhängigen Variablen, Koeffizienten der Regressionsgeraden mit statistischen Kenngrößen. Regressionsverfahren $=$ schrittweise. Auswahl: Lehmböden

Tab. 10C

Ergebnisse der linearen multiplen Regressionsanalyse zwischen unabhängigen und abhängigen Variablen, Koeffizienten der Regressionsgeraden mit statistischen Kenngrößen. Regressionsverfahren = schrittweise. Auswahl: Sandböden

Tab. 11

Flächenmittelwerte der berechneten Messgrößen in \% der Messwerte; absolute Differenzen zwischen gemessenen und berechneten-werten in Klammern; $\mathrm{R}^{2}=\mathrm{Be}-$ stimmtheitsmaß für die Korrelation zwischen gemessenen und berechneten Flächenmittelwerten

Tab. 12

Ergebnisse der Modellierung des langfristigen Nitratabbaus in den untersuchten Böden 


\section{Verzeichnis der Tabellen in Anhang}

Anhang 1

Tiefenverteilung der bodenchemischen Parameter sowie der Korngrößenzusammensetzung der untersuchten Auen-Böden. Mittelwert, \pm Standardabweichung, bei Anzahl $=2$ Differenzen in Klammern

Anhang 2

Tiefenverteilung der bodenchemischen Parameter sowie der Korngrößenzusammensetzung der untersuchten Sand-Böden. Mittelwert, \pm Standardabweichung, bei Anzahl $=2$ Differenzen in Klammern

Anhang 3

Tiefenverteilung der bodenchemischen Parametern sowie der Korngrößenzusammensetzung der untersuchten Seemarsch. Mittelwert, \pm Standardabweichung, bei Anzahl $=2$ Differenzen in Klammern

Anhang 4

Zusammenfassung der Ergebnisse zur Reduktion von Nitrat und Produktion von Sulfat aus den anaeroben und aeroben Inkubationsversuchen: Schichtmittelwerte der Umsatzraten zu Versuchsbeginn $\left(\mathrm{R}_{\mathrm{NO} 3}\right.$, DR $\left.\left({ }^{15} \mathrm{~N}_{2}+\mathrm{N}_{2} \mathrm{O}\right), \mathrm{R}_{\mathrm{SO} 4}\right)$ kumulierte Umsetzungen $\left(\mathrm{M}_{\mathrm{NO} 3}, \mathrm{M}_{\mathrm{SO} 4}\right)$ Anteil der heterotrophen Denitrifikation am Gesamtumsatz $\left(\mathrm{M}_{\mathrm{NO} 3} \mathrm{H}: \mathrm{M}_{\mathrm{NO} 3(\mathrm{ges})}\right)$, Nachhaltigkeits- und Verfügbarkeits-Indizies (NI, VI)

\section{Anhang 5}

Ergebnisse der linearen multiple Regressionsanlyse zwischen unabhängigen und abhängigen Koeffizienten der Regressionsgeraden mit statistische Kenngrößen 


\section{Verzeichnis häufig verwendeter Abkürzungen}

$\begin{array}{ll}\mathrm{C}_{\mathrm{hwl}} & \text { Heißwasserlöslischer Kohlenstoff } \\ \mathrm{C}_{\text {lös }} & \text { Gelöster organischer Kohlenstoff } \\ \mathrm{C}_{\mathrm{NO} 3 \mathrm{r}} & \begin{array}{l}\text { Restnitratkonzentration des Grundwassers nach Passage der } \\ \text { reaktiven Schicht }\end{array} \\ \mathrm{d} & \text { Tag } \\ \mathrm{D} & \text { Schichtmächtigkeit } \\ \mathrm{DR}^{15}\left(\mathrm{~N}_{2}+\mathrm{N}_{2} \mathrm{O}\right) & { }^{15} \mathrm{~N}-\text { Denitrifikationsrate, ermittelt aus der }{ }^{15} \mathrm{~N}-\text { Gasfreisetzung } \\ \mathrm{dZ} & \text { Vertikalkomponente } \\ \mathrm{E} & \text { Porosität } \\ \mathrm{Fe}_{\text {lös }} & \text { Gelöstes Eisen } \\ \mathrm{F}_{\mathrm{NO} 3} & \text { Jährliche Nitratfracht } \\ \mathrm{GW} & \text { Grundwasserflurabstand } \\ \text { Kap. } & \text { Kapitel }\end{array}$

$\mathrm{M}_{\mathrm{NO} 3}$

Kumulierte Menge der Nitratreduktion während der Inkubationszeit

$\mathrm{M}_{\mathrm{NO} 3 p o t}$

$\mathrm{M}_{\mathrm{SO} 4}$

$\mathrm{M}_{\mathrm{NO} \text { eff }}$

Potentielle Nitratabbaumenge

Kumulierte Menge der Sulfatproduktion während der Inkubationszeit

$\mathrm{M}_{\mathrm{NO} 3} \mathrm{H}: \mathrm{M}_{\mathrm{NO} 3 \text { (ges) }}$

Effektive Nitratabbaumenge

Verhältnis „heterotrophe Denitrifikation zu GesamtDenitrifikation“"

$\mathrm{N}$

Anzahl der Proben

n.b.

nicht bestimmt

NI

Nachhaltigkeitsindex

n.n.

nicht nachweisbar

n.s.

nicht signifikant

PVC

Polyvinylchlorid

PE

Polyethylen

$\mathrm{R}_{\mathrm{NO} 3}$

Denitrifikationsrate zu Versuchsbeginn ermittelt duch Nitratverbrauch

$\mathrm{T}_{\mathrm{V}} \quad$ Verweilzeit

VI Verfügbarkeitsindex

$\pm \quad$ Standardabweichung 
Zusatzsymbole für die gemessenen Kenngrößen (tiefgestellt, nachgestellt oder in Klammern)

$\begin{array}{ll}\text { A } & \text { Aerobe Sulfatproduktion } \\ \mathrm{H} & \text { Heterotrophe Denitrifikation } \\ \mathrm{L} & \text { Lehm } \\ \mathrm{NO}_{3} & \text { Nitrat } \\ \mathrm{S} & \text { Sand } \\ 500 \mathrm{~d} & \text { Bezogen auf Inkubationszeit von } \mathrm{T}_{0} \text { bis } \mathrm{T}_{500} \\ (\infty) & \text { Bezogen auf unendliche Inkubationszeit }\end{array}$




\section{Einleitung und Problemstellung}

In der Bundesrepublik Deutschland stellt die Stickstoffdynamik in Agrarökosystemen ein komplexes Umweltproblem dar. Eine Messgröße für das GesamtStickstoffbelastungspotential ist der flächenbezogene Bilanzsaldo, d.h. die Differenz zwischen den Einträgen und der Abfuhr mit landwirtschaftlichen Produkten. So wurde z.B. in einer Bilanz für die landwirtschaftlichen Betriebe der alten Bundesländer im Zeitraum 1982-1992 ein N-Überschuss aus den landwirtschaftlichen Betrieben im Mittel von $103 \mathrm{~kg} \mathrm{~N} \mathrm{ha}^{-1} \mathrm{a}^{-1}$ mit fallender Tendenz ( $88 \mathrm{~kg} \mathrm{~N}^{-1}$ in 1991) berechnet (SCHEFFER \& SCHACHTSCHABEL, 2002). WENDLAND et al. (1993) geben für die Bundesrepublik Deutschland mittlere Stickstoff-Salden von ca. 86 $\mathrm{kg} \mathrm{N}$ pro ha und Jahr an, wobei in Gebieten mit verbreiteter flächenunabhängiger tierischer Veredlung (z.B. Vechta, Cloppenburg, Ostwestfalen, Münsterland) mehr als doppelt so hohe Beträge auftreten wie in Gebieten ertragreicher Böden (z.B. Lössböden) mit intensiven Anbau von Marktfrüchten (SCHEFFER \& SCHACHTSCHABEL, 2002). Der N-Überschuss in den neuen Bundesländern liegt im Durchschnitt auf annähernd gleicher Höhe wie in den alten Bundesländern Deutschlands. Diese N-Überschüsse können die Umwelt auf verschiedenen Wegen belasten. Ein Teil des N-Saldos wird durch Denitrifikation mit partieller $\mathrm{N}_{2} \mathrm{O}-$ Bildung und durch Ammoniakentbindung freigesetzt und trägt somit zur Belastung der Atmosphäre und zur N-Eutrophierung naturnaher Ökosysteme bei. Sofern keine Änderungen des N-Vorrates im Boden stattfinden, wird die verbleibende N-Fracht als Nitrat mit dem Sickerwasser ausgewaschen.

Der Nitrateintrag in das Grundwasser ist hauptsächlich auf den intensiven Einsatz von stickstoffhaltigen Düngemitteln zurückzuführen, weshalb in vorrangig landwirtschaftlich genutzten Regionen die Nitratbelastung des Grundwassers häufig besonders hoch ist. Dadurch ergeben sich auch regional große Unterschiede. Durch die Nitratverlagerung in die Grundwasserzone besteht für die Trinkwassergewinnung das Problem, den EG-Grenzwert von $50 \mathrm{mg}$ Nitrat $1^{-1}$ einzuhalten. In dieser Hinsicht ist die Denitrifikation als günstiger Prozess zu beurteilen, da in vielen Fällen ein bedeutender Anteil der Nitratfracht in der ungesättigten Zone und im Grundwasser abgebaut wird. 
Die Denitrifikation nimmt eine zentrale Rolle im N-Kreislauf von Agrarökosystemen ein (Abb. 1). Sie ist ein vorwiegend biochemischer Prozess, der bei hoher Wassersättigung der Böden (oberhalb 70 - $80 \%$ des gesamten Porenvolumens) bzw. eingeschränkter Durchlüftung bevorzugt abläuft (SCHEFFER \& SCHACHTSCHABEL, 2002). Dabei verläuft die Reduktion von Nitrat und Nitrit zu gasförmigem Distickstoff $\left(\mathrm{N}_{2}\right)$ und Distickstoffoxid $\left(\mathrm{N}_{2} \mathrm{O}\right.$, Lachgas) nach dem in Abb. 1 gezeigten Reaktionsschema.

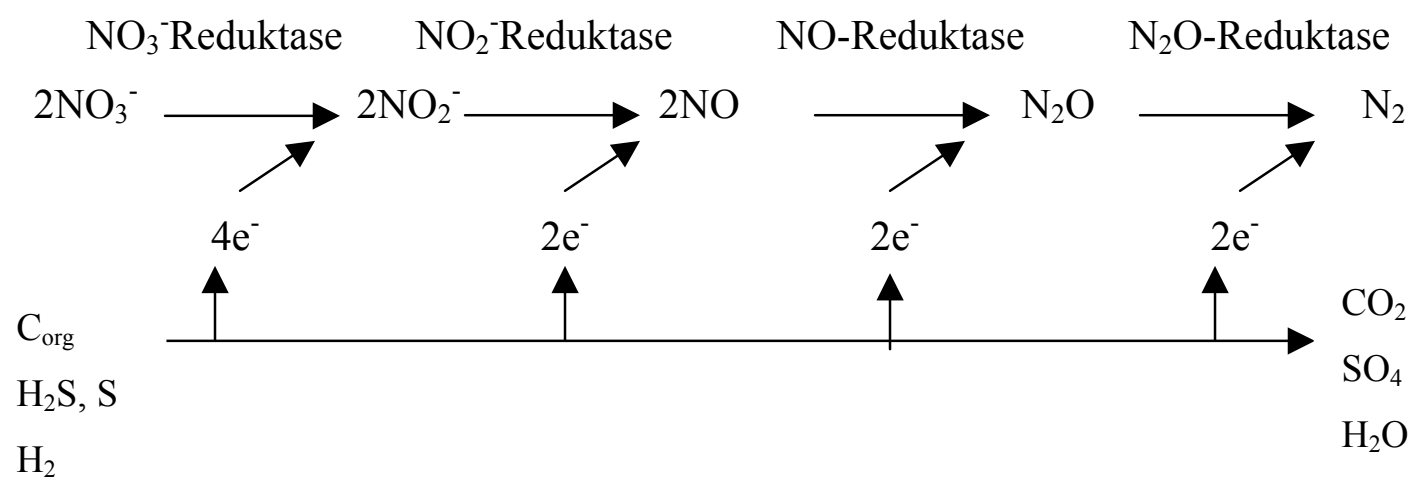

\section{Abb. 1: Schritte der Nitratreduktion zu $\mathbf{N}_{2}$, beteiligte Substrate und Produkte (nach BECKER et al., 1992)}

Für die in der Regel gut belüfteten landwirtschaftlichen Böden in Mitteleuropa werden maximale $\mathrm{N}$-Reduktionsraten von ca. $25 \mathrm{~kg} \mathrm{~N} \mathrm{ha}^{-1} \mathrm{a}^{-1}$ angenommen (NIEDER et al., 1989). Im Mittel sind das 15 \% der eingesetzten Düngermenge. Die weniger gut belüfteten hydromorphen Böden mit ausreichender Verfügbarkeit der Reduktionsmittel wie z.B. organischer Kohlenstoff und Eisen-Schwefel-Verbindungen weisen verstärkte Denitrifikationraten auf, nämlich von ca. 50 bis über $150 \mathrm{~kg} \mathrm{~N} \mathrm{ha}^{-1} \mathrm{a}^{-1}$ (GÄTH et al., 1997). Für landwirtschaftlich genutzte Böden wurden N-Verluste von 40-100 kg N ha ${ }^{-1} \mathrm{a}^{-1}$ berichtet (SCHEFFER \& SCHACHTSCHABEL 2002). In schluffigbindigen Böden mit hohem Grundwasserstand (z.B. Marsch-Böden) wurden beispielsweise Denitrifikationsraten von $96 \mathrm{~kg} \mathrm{~N} \mathrm{ha}^{-1} \mathrm{a}^{-1}$ und in Böden mit hoher Gülledüngung unter Acker von 36-65 $\mathrm{kg} \mathrm{N} \mathrm{ha}^{-1} \mathrm{a}^{-1}$ gemessen (SCHEFFER \& SCHACHTSCHABEL, 2002). 


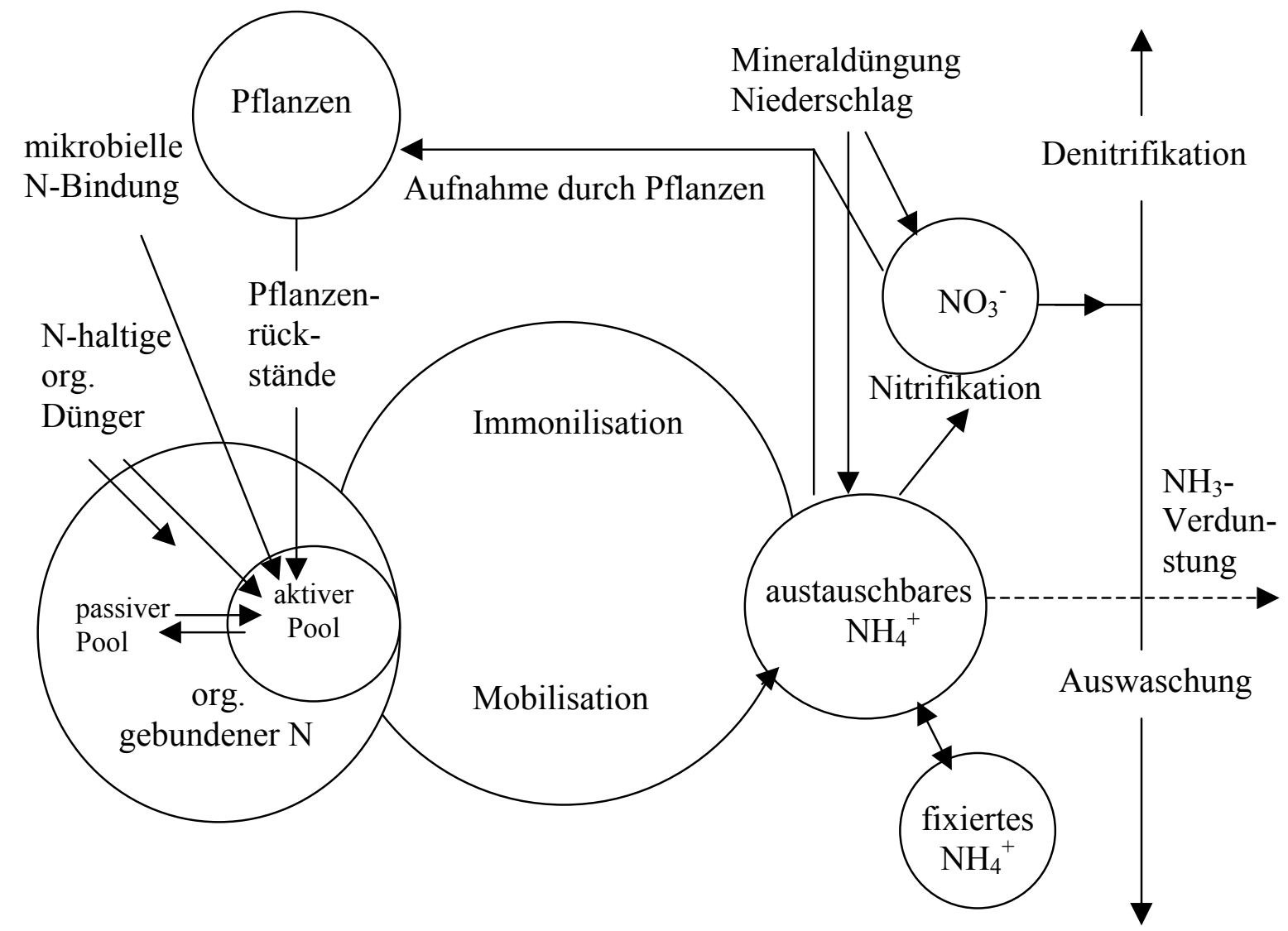

\section{Abb. 2: Der landwirtschftliche N-Kreislauf (verändert nach S. L. JANSSON, in SCHEFFER \& SCHACHTSCHABEL, 2002)}

Voraussetzung für den mikrobiellen Denitrifikationsprozess ist das Zusammentreffen von Nitrat, Anaerobie und geeigneten Reduktionsmitteln (vgl. Abb. 1). Bei der Beurteilung der Denitrifikationskapazität stellt sich die Frage, ob die vorhandenen Reduktionsmittel sich regenerieren, wie z.B. der organische Kohlenstoff des Wurzelraums, oder ob es sich um fossile Quellen handelt, wie z.B. Anreicherungen von reduzierten Eisen-Schwefel-Verbindungen oder von fossilem organischem Kohlenstoff im Untergrund. Ist letzteres der Fall, dann führen anhaltende Nitratausträge langfristig zu einer Erschöpfung des Nitrat-Abbaupotentials und irgendwann letztlich zum Durchbruch des Nitrats über das Grundwasser in Trinkwasserentnahmestellen und Offengewässer. Obwohl dieses Problem schon seit längerer Zeit diskutiert wird (WENDLAND et al., 1993; OBERMANN, 1989), gab es bisher kaum quantitative Untersuchungen, aus denen sich die Abbaurate der verschiedenen Formen fossiler Reduk- 
tionsmittel ableiten ließe. Somit ist bisher keine zuverlässige Prognose für den Prozess der irreversiblen Erschöpfung des Denitrifikationspotentials möglich. Auch existierten keine quantitativen Erkenntnisse zu der Frage, welcher Anteil der erschöpfbaren Reduktionsmittel seit der Intensivierung der N-Düngung bereits verbraucht ist. Zur Verbesserung des langfristigen Grundwasserschutzes ist jedoch eine Erweiterung des Kenntnisstandes zu diesen Fragestellungen notwendig.

Ziel der vorliegenden Arbeit ist es, quantitative Aussagen über den denitrifikativen Abbau der verschiedenen Formen fossiler Reduktionsmittel im oberflächennahen Grundwasser hydromorpher Böden zu treffen. Somit soll die Erschöpfbarkeit des Denitrifikationspotentials in diesen Böden bestimmt werden.

\section{Stand der Forschung}

\subsection{Denitrifikationsprozesse in wasserungesättigten Böden}

Sowohl das Auftreten, als auch das Ausmaß der Denitrifikation in Böden ist von bestimmten Milieubedingungen abhängig. Die Denitrifikation erfolgt in Boden und Grundwasser vorwiegend als dissimilatorische Reduktion von Nitrat $\left(\mathrm{NO}_{3}{ }^{-}\right)$. Dabei dient Nitrat unter anaeroben Bedingungen als terminaler Elektronenakzeptor der mikrobiellen Energiegewinnung (vgl. Abb. 1). Neben der mikrobiellen Denitrifikation ist grundsätzliche auch eine chemische Denitrifikation möglich (HOFFMANN, 1991). Die dissimilatorischen Nitratreduktionen unterscheiden sich weiterhin auch in der Art des beteiligten Elektronendonators: (a) die organotrophe oder heterotrophe Denitrifikation, (b) die chemolithoautotrophe Denitrifikation.

(a) Organotrophe Denitrifikation: Die Denitrifikanten gewinnen ihre Energie aus der Oxidation organischer Substanz. Nach HofFMANN (1991) benutzen Bakterien zur organotrophen oder heterotrophen Denitrifikation organische Substanzen sowohl als Wasserstoff- (organotroph) als auch Kohlenstoffquellen (heterotroph). Der Prozess lässt sich folgendermaßen darstellen (BURFORD \& BREMNER, 1975; TRUDELL et al., 1996):

$$
5 \mathrm{CH}_{2} \mathrm{O}+4 \mathrm{NO}_{3}^{-}+4 \mathrm{H}^{+} \longrightarrow 2 \mathrm{~N}_{2}+5 \mathrm{CO}_{2}+7 \mathrm{H}_{2} \mathrm{O}
$$


Der Kohlenstoff ist für heterotrophe Mikroorganismen nicht nur als Elektronendonator notwendig, sondern dient auch dem assimilatorischen Stoffwechsel. Nur ein geringer Teil des organischen Kohlenstoffs ist für die organotrophen Organismen verfügbar.

(b) Chemolithoautotrophe Denitrifikation: Bei diesem Stoffwechselweg verwenden die Denitrifikanten dagegen anorganische Substanzen als Elektronendonator $\left(\mathrm{S}_{2}{ }^{-}, \mathrm{S}^{0}, \mathrm{Fe}^{2+}\right)$ und Kohlendioxid $\left(\mathrm{CO}_{2}\right)$ als C-Quelle. Als Pyrit, Markasit oder Pseudomorphosen (petrifizierte Umwandlungsprodukte abgestorbener Mikroorganismen) vorliegende Schwefelverbindungen werden bei der $\mathrm{FeS}_{2}-$ Denitrifikation unter Beteiligung von Nitrat zu Sulfat aufoxidiert (KöLlE, 1985; BötTCher, 1992; Buresh \& Moraghan, 1976; Lind \& Pedersen, 1976). Die Reaktion verläuft nach KöLLE et al. (1985) gemäß folgender Gleichung:

$$
5 \mathrm{FeS}_{2}+14 \mathrm{NO}_{3}{ }^{-}+4 \mathrm{H}^{+} \longrightarrow 7 \mathrm{~N}_{2}+10 \mathrm{SO}_{4}{ }^{2-}+5 \mathrm{Fe}^{2+}+2 \mathrm{H}_{2} \mathrm{O}
$$

Unter sauren Bedingungen ist auch eine Chemo-Denitrifikation möglich. ChemoDenitrifikation ist gekennzeichnet durch eine Vielzahl von möglichen Reaktionen des Nitrits, die häufig bei niedrigem pH-Wert ablaufen (HofFMAnN, 1993). Die Bedeutung der Chemo-Denitrifikation im Boden für Nitritzerfall ist bisher nicht geklärt (CHALK \& SMITH, 1983). Unter Feldbedingungen liegen keine Abschätzungen von N-Verlustmengen aus der Chemo-Denitrifikation vor.

\subsection{Bedeutung der Denitrifikation}

Die Denitrifikation spielt auch im globalen Stickstoffkreislauf eine große Rolle. Würde Stickstoff in Form von $\mathrm{NO}_{3}{ }^{-}$nicht in die Atmosphäre zurückgeführt, würde diese langsam an $\mathrm{N}_{2}$ verarmen und das $\mathrm{NO}_{3}^{-}$würde sich letztendlich im Meer anhäufen (SCHLEGEL, 1985). Nitrat stellt die wichtigste pflanzenverfügbare Stickstoffform dar, kann aber auch sehr leicht in tiefere Bodenschichten verlagert und schließlich ausge- 
waschen werden. Der Hauptaspekt der Denitrifikation ist der Abbau von Nitrat. Dadurch erfolgt einerseits eine Erschwerung der N-Ernährung der Kulturpflanzen, zum anderen eine Verminderung der Nitratfracht des Sickerwassers und somit der Grundwasserbelastung. Dem Nitratabbau im oberflächennahen Grundwasser kommt eine besondere Rolle zu, da hier durch das Zusammentreffen von hohem Kohlenstoff-Angebot und limitierter $\mathrm{O}_{2}$-Verfügbarkeit sehr hohe Aktivitäten auftreten können.

Die Untersuchungen der letzten Jahre ergaben, dass die Denitrifikation nicht nur im Oberboden sondern auch in der Dränzone (BECKER \& MEYER, 1987; Well, 1993), im Bereich des Kapillarsaums des Grundwassers (SCHEFFER \& SCHACHTSCHABEL, 2002, BEÖCZY, 1988a; DRECHSLER, 1992) und im Bereich des Grundwasserleiters (OBERMANN, 1981; KÖLLE, 1983; WoLfF et al., 1985, WELL et al., 2001, 2003) ablaufen kann. Für die Belastung der Atmosphäre spielen die gasförmigen Zwischenprodukte der Denitrifikation, die Stickstoffoxide $\mathrm{NO}$ und $\mathrm{N}_{2} \mathrm{O}$ (Lachgas) eine Rolle. $\mathrm{N}_{2} \mathrm{O}$ ist an der Ozonzerstörung in der Stratosphäre beteiligt (SEILER \& CONRAD, 1981; BOUWMAN, 1990). Zusätzlich gehört $\mathrm{N}_{2} \mathrm{O}$ zu den Treibhausgasen und ist deshalb für den anthropogen Treibhauseffekt mitverantwortlich (SAUERBECK \& ISERMANN, 1993). Der Treibhauseffekt beruht darauf, dass einfallende, kurzwellige Sonnenstrahlung von der Erdoberfläche als langwellige Wärmestrahlen wieder abgegeben wird und von den infrarotabsorbierenden Gasen der Atmosphäre zurückgehalten werden.

AULAKH et al., 1992 berichten, dass der relative Anteil des Lachgases an den gesamten gasförmigen N-Verlusten denitrifikativer Herkunft zwischen 0 und $100 \%$ variieren kann.

Insgesamt trägt $\mathrm{N}_{2} \mathrm{O}$ zu ca. $6 \%$ am derzeitigen anthropogenen Treibhauseffekt bei (IPCC, 1997). Die Zunahme der $\mathrm{N}_{2} \mathrm{O}-$ Konzentration in der Atmosphäre von etwa 295 ppb seit Mitte dieses Jahrhunderts auf 310 ppb (KHALIL \& RASMUSSEN, 1988) ist auf die zunehmende anthropogene Emission dieses Spurengases zurückzuführen. Die Ursache ist neben der Verbrennung fossiler Brennstoffe im Energie- und Verkehrssektor und der Rodung von Wäldern in erster Linie in der Intensivierung der Landwirtschaft zu sehen (USEPA, 1989). 


\section{3 Ökologische Voraussetzungen für die Denitrifikation}

Die wesentlichen Einflussgrößen der Denitrifikation im Boden sind nach KNOWLES (1982), Heyder et al. (1985), Schulte-Kellinghaus (1987), Nieder et al. (1989) und HOFFMANN (1991):

- Verfügbarkeit von Reduktionsmitteln

- Nitratkonzentration

- Vorhandensein denitrifizierender Mikroorganismen

- Anaerobie/ $\mathrm{O}_{2}$-Partialdruck bzw. Wassergehalt

- $\mathrm{pH}-\mathrm{Wert}$

- Temperatur

\subsubsection{Einfluss von Reduktionsmitteln}

\section{a) Organischer Kohlenstoff $\left(\mathrm{C}_{\text {org }}\right)$}

Die Denitrifikation wird von der Menge und Verfügbarkeit der im Boden bzw. Aquifer vorhandenen organischen Substanz gesteuert (BOWMAN \& FOCHT, 1974). Als Energiematerial (Elektronendonator) und für die Synthese von Zellbestandteilen der Mikroorganismen ist leicht verfügbarer organischer Kohlenstoff notwendig, wie er in nativer organischer Bodensubstanz sowie vor allem in Ernterückständen, Gründüngung, organischen Wirtschaftsdüngern sowie Wurzelexudaten (besonders bei engem $\mathrm{C} / \mathrm{N}$ Verhältnis) enthalten ist (Scheffer \& Schachtschabel, 2002, Bowman \& Focht, 1974). Eine Erhöhung des leicht verfügbaren Kohlenstoffs im Boden wird auch durch häufige und intensive Durchfeuchtungs- und Trockenzyklen bzw. Frieren und Auftauen bewirkt (SCHEFFER \& SCHACHTSCHABEL, 2002; RYDEN et al., 1979; BOWMAN \& FOCHT, 1974). Bei hohen Gehalten von leicht verfügbarem organischem Kohlenstoff in den Mikroräumen von aggregierten Böden kann es auch zu einer bedeutenden Denitrifikation unterhalb eines Wassergehalts von 60-70 \% des gesamten Porenvolumens kommen (SCHEFFER \& SCHACHTSCHABEL, 2002).

Die mikrobiell verfügbare organische Substanz wirkt zudem indirekt auf die Nitratatmung, da heterotrophe aerobe Organismen durch die $\mathrm{O}_{2}$-Zehrung ein anaerobes Milieu schaffen können (CHALAMET, 1985). 
In pleistozänen Grundwasserleitern liegt der organische Kohlenstoff meist als partikuläre organische Substanz, als Torf-, Braunkohle- oder Holzstücke vor. Nur ein geringer Teil des organischen Kohlenstoffes ist im Grundwasser gelöst (HoFFMANN, 1991). LEUCHS (1988) hat in einem Aquifer einen Gehalt an gelöstem organischen Kohlenstoff zwischen 2 und $15 \mathrm{mg} \mathrm{l}^{-1}$, einen Anteil der partikulären organischen Substanz am Sediment von 1,5 Gew.\% und einen Anteil des fein dispers verteilten organischen Kohlenstoffs von 0,01 bis 0,3 Gew.\% im Grundwasser gemessen.

Bei Vorhandensein von Nitrat stellt die Menge an organischer Substanz unter anaeroben Bedingungen den wichtigsten limitierenden Faktor für die Denitrifikation dar (KNOWLES 1982; REDDY et al., 1982; El-DEMERDASH \& OTTOW 1983).

\section{b) Schwefelverbindungen}

Bei der chemolithoautotrophen Denitrifikation dienen den Mikroorganismen primäre Schwefelverbindungen wie Pyrit oder Markasit (beide $\mathrm{FeS}_{2}$ ) und Mackinawit (FeS) (SCHEFFER \& SCHACHTSCHABEL, 2002) sowie amorphe sulfidische Sekundärbildungen als Energiequelle (BÖTTCHER, 1992). In Böden des humiden Klimabereichs kann der SGehalt $0,1-0,5 \mathrm{~g} \mathrm{~kg}^{-1}$, in Mooren bis $\mathrm{zu} 10 \mathrm{~g} \mathrm{~kg}^{-1}$ und in sulfatsauren Marschen bis zu

$35 \mathrm{~g} \mathrm{~kg}^{-1}$ betragen. Unter reduzierenden Bedingungen kann eine S-Anreicherung in Form von Eisensulfiden erfolgen, wenn Böden oder Sedimenten mit dem Grund- oder Überflutungswasser Sulfate zugeführt werden (SCHEFFER \& SCHACHTSCHABEL, 2002).

Die reduzierten Eisen-Schwefelverbindungen können im Aquifer als Komponente von Braunkohle, als dünne reaktionsfähige Überzüge in Form von Kristallen auf Kies- oder Sandkörnern oder als selbständige Aggregate vorliegen (KöLLE et al., 1985). Der Anteil der Eisensulfide am Sediment liegt häufig im Bereich zwischen 0,01 und 0,1 \% (KöLLE, 1987/1988). Chemolithoautotrophe Denitrifikation wurde in verschiedenen Grundwassereinzugsgebieten schon beobachtet (KÖLLE et al., 1983; VAN BEEK et al., 1988, PostMa et al., 1991).

\subsubsection{Einfluss der Nitratkonzentration}

Die Bedingungen für die Denitrifikation sind standortbedingt und deshalb ist die Reaktionskinetik ein variables Merkmal (AвOU-SEADA \& OTTOW, 1988). Die Denitrifikation 
folgt im Boden unter anaeroben Bedingungen und bei hohen Nitrat-Konzentrationen einer Funktion nullter Ordnung und verläuft somit unabhängig vom Nitratgehalt (ELDemerdash \& Ottow, 1983; Chalamet, 1985; Payne, 1985; Starr \& Parlange 1975; FOCHT, 1974). Bei niedriger Nitratkonzentration $\left(<20-40\right.$ ppm $\left.\mathrm{NO}_{3}\right)$ wird Nitrat aufgrund von Diffusionsproblemen zum begrenzenden Faktor des Stoffwechsels $(<40$ $\mathrm{mg} \mathrm{NO}_{3}-\mathrm{N}^{-1}$ ). Dann folgt die Denitrifikation einer Reaktionen 1. Ordnung (REDDY et al., 1982; STANFORD et al., 1975). Theoretisch wird Nitrat bei einer vollständigen Denitrifikation nicht zum begrenzenden Faktor, solange das molare Verhältnis von Wasserstoffdonator zu Nitrat nicht weiter als 5:1 (Donator : Akzeptor) ist, da die Oxidationsstufen von Nitrat und molekularem Stickstoff um fünf verschieden sind (ABOU-SEADA \& OTTOW, 1988).

Auch die Zusammensetzung der Stickstoffentgasung wird von der Nitratkonzentration beeinflusst. Eine Verstärkung des relativen $\mathrm{N}_{2} \mathrm{O}$-Anteils mit zunehmendem Nitratgehalt wurde von Blackmer \& Bremner, 1978, Cho \& SAKdinan, 1978, Firestone et al., 1980; TERRY \& TATE, 1980; RYDEN, 1983 und NÖMMIK et al., 1984 berichtet.

\subsubsection{Vorhandensein denitrifizierender Mikroorganismen}

Die Denitrifikanten zählen zu den fakultativen Anaerobiern, die ihren Stoffwechsel bei Sauerstoffmangel von Sauerstoffatmung auf Nitratatmung umstellen können. Bei einem großen Teil der ubiquitären Bakterien (1 - 10\%) ist die potentielle Fähigkeit zur Denitrifikation gegeben (SCHMIDER \& OTTOW, 1984), so dass die Grundvoraussetzung zur Nitratatmung in Böden stets vorhanden ist (ABOU-SEADA, 1986). Zwar nimmt mit zunehmender Bodentiefe der Besatz an Mikroorganismen ab, doch kann davon ausgegangen werden, dass in der Regel die Präsenz der Mikroorganismen keinen begrenzenden Faktor darstellt (SCHUlTE-Kellinghaus, 1987; EILAND \& LiND, 1987; Hoos \& SchWEIßFuRTH, 1982; Whitelaw \& REES, 1980).

Die häufigsten Vertreter von Denitrifikanten sind Bakterien der Gattung Pseudomonas, Alcaligenes, Bacillus, Thiobacillus denitrificans, Corynebacterium, Paracoccus, Chromobacterium und Spirillum (KNOWLES, 1982; GERMON, 1985 \& PAYNE, 1985). MALINOWSKY \& OTTOW (1985) haben Denitrifikation durch einige Pilzarten u.a. Fusarium, Acremonium und Aspergillus beobachtet. Nitratreduktion durch reduzierte 
Schwefel-Verbindungen wurde bei Thiobacillus denitrificans (KÖLLE et al., 1983) und durch $\mathrm{Fe}^{2+}$ bei Gallionella ferruginea (PosTMA, 1990) festgestellt.

\subsubsection{Einfluss des $\mathrm{O}_{2}$-Partialdruckes bzw. Wassergehaltes im Boden}

Bei der dissimilatorischen Nitratreduktion dient $\mathrm{NO}_{3}{ }^{-}$als terminaler Elektronenakzeptor für die mikrobielle Energiegewinnung. Da Nitrat erst bei vermindertem Sauerstoffangebot als Elektronenakzeptor Verwendung findet, kommt der Sauerstoffverfügbarkeit und damit den Bodenwassergehalten entscheidende Bedeutung zu. Hohe Denitrifikationsverluste wurden in Feldversuchen bei Bodenfeuchten über $80 \%$ der Feldkapazität gemessen (RoLSTON et al., 1984; BENCKISER et al., 1986). Die O $2^{-}$

Diffusion ist in Wasser gegenüber Luft etwa um den Faktor $10^{4}$ herabgesetzt (FocHT, 1981). Bei steigenden Bodenwassergehalte wird die Denitrifikation daher sowohl durch eine Verringerung der $\mathrm{O}_{2}$-Diffusion im Boden als auch durch einen gesteigerten mikrobiellen Sauerstoffverbrauch in Folge der Zersetzung leicht verfügbarer organischer Substanz gefördert. Bei erhöhten Gehalten an leicht verfügbarem Kohlenstoff wird die mikrobielle Aktivität derart erhöht, dass der Sauerstoffbedarf der Mikroorganismen die Sauerstoffnachlieferung durch Diffusion überschreitet und damit $\mathrm{O}_{2}$-arme bzw. -freie Mikroräume entstehen, in denen eine Denitrifikation erfolgt (SCHEFFER \& SchACHTSCHABEL, 2002).

Der Einfluss der $\mathrm{O}_{2}$-Konzentration auf die Nitratreduktion wird u.a. von MisRA et al. (1974c); Firestone et al. (1979); Binnerup et al. (1992); Abou-SEADA \& OtTOW (1985); OtTOW \& El DeMERDASh (1983); LeFFlAAR (1986); KANWAR et al. (1982); TIEDJE et al. (1984) und ROLSTON et al. (1976) beschrieben.

\subsubsection{Einfluss des pH-Wertes}

Die Mehrzahl der Denitrifikanten verzeichnet im neutral bis schwach alkalischen pH-Milieu (pH 7-8) im allgemeinen die höchste Denitrifikationsintensität (BREMNER \& Shaw, 1985; KnOWLeS, 1982; Riss \& SCHWEISFurTh, 1985). Meistens nimmt die Denitrifikationsrate außerhalb des neutralen bis schwach alkalischen $\mathrm{pH}$-Bereichs 
stark ab (ABOU-SEADA, 1986). In sauren Böden (um pH 3,5) konnten jedoch noch messbare Stickstoffentgasungen ermittelt werden (KLEMEDTSSON et al., 1977; MÜLLER et al., 1980; PARKIN et al., 1985a). Häufig wird berichtet, dass die Denitrifikation im sauren Milieu unvollständig verläuft, und dadurch findet eine bevorzugte $\mathrm{N}_{2} \mathrm{O}$-Freisetzung statt. Hingegen soll es im neutralen bis schwach alkalischen Milieu eher zu einer vollständigen Reduktion bis zu N 2 kommen (FOCHT, 1974; BLACKMER \& Bremner, 1978; Firestone et al., 1980; Rosswall, 1982; VAn Cleemput, 1984).

Aufgrund bestehender artspezifischer pH-Optima der Mikroorganismen (BURTH \& OTтоw, 1982) dürfte im meist schwach sauren bis schwach alkalischen pH-Bereich der Ackerböden keine Einschränkung der mikrobiellen Denitrifikation gegeben sein.

\subsubsection{Einfluss der Temperatur}

Die Denitrifikation läuft darüber hinaus in einem weiten Temperaturbereich zwischen $5^{\circ} \mathrm{C}$ und bei Maximumwerten im Bereich von $65-75^{\circ} \mathrm{C}$ ab (SCHEFFER \& SCHACHTSCHABEL, 2002). Der Temperatur-Einfluss auf die Denitrifikation ist schwierig zu beurteilen, da die Temperatur sowohl auf die gesamte biologische Aktivität als auch auf die Sauerstoff-Löslichkeit und die $\mathrm{O}_{2}$-Diffusion in Boden und Grundwasser wirkt (Hoffman, 1991). Das Optimum befindet sich bei ca. $37^{\circ} \mathrm{C}$ (Lensi \& ChalameT, 1982). In Laborversuchen wurde unterhalb von $2^{\circ} \mathrm{C}$ (BREMNER \& SHAW, 1958), $3^{\circ} \mathrm{C}$ (NÖMmiK, 1956), $4^{\circ} \mathrm{C}$ (Lensi \& Chalamet, 1982) bzw. $5^{\circ} \mathrm{C}$ (Bailey \& BeAuChamp, 1973) keine Nitratatmung mehr beobachtet. In Feldversuchen wurden in Mineralböden erst messbare Raten bei Temperaturen über $5-8^{\circ} \mathrm{C}$ (KNOWLES, 1982), bzw. über $15^{\circ} \mathrm{C}$ (BENCKISER et al., 1986; \& FREDE et al., 1975) erreicht. WELL et al. (2001) stellten in einem organischen Boden schon bei $2^{\circ} \mathrm{C}$ hohe Denitrifikationsraten fest. Da die durchschnittlichen Jahres-Temperaturen in $2 \mathrm{~m}$ Tiefe in Mitteleuropa etwa $10^{\circ} \mathrm{C}$ betragen, können im tieferen Untergrund und im Grundwasser keine vollständig hemmenden Wirkungen für die Denitrifikation infolge niedriger Temperaturen auftreten.

\subsection{Prozesse des Nitratabbaus in hydromorphen Böden}

Die fakultativ anaeroben Denitrifikanten reduzieren nur dann Nitrat, wenn Sauerstoff nicht in ausreichendem Maße zur Verfügung steht. Dies beruht darauf, dass die Ni- 
tratreduktion mit einer geringeren Energieausbeute verbunden ist als die $\mathrm{O}_{2}$-Reduktion (TURNER \& PATRICK, 1968). So wird im Boden theoretisch ein Redoxsystem niedrigeren Energieniveaus induziert, wenn die Umsetzungen höheren Niveaus durch den Abbau eines Reaktionspartners weitgehend beendet sind. In Tab. 1 sind theoretisch berechnete und experimentell ermittelte Daten für verschiedene Redoxreaktionen angegeben.

Tab. 1: Vergleich experimentell ermittelter und berechneter Redoxpotentialle für verschiedene Redoxreaktionen bei pH 7 (nach SCHEFFER \& SCHACHTSCHABEL, 1992 in ROLLAND, 1995)

\begin{tabular}{lll}
\hline Redoxreaktion & $\begin{array}{l}\text { Eh }(\mathbf{m V}) \\
\text { experimentell }\end{array}$ & $\begin{array}{l}\text { Eh (mV) } \\
\text { berechnet }\end{array}$ \\
\hline Beginn der $\mathrm{O}_{2}$-Reduktion & - & 813 \\
Beginn der $\mathrm{NO}_{3}{ }^{-}$-Reduktion & $450-550$ & 746 \\
Beginn der $\mathrm{Mn}^{2-}$-Bildung & $350-45$ & 396 \\
Beginn der Ammonifikation & - & 363 \\
$\mathrm{O}_{2}$ nicht mehr nachweisbar & 330 & - \\
$\mathrm{NO}_{3}$ nicht mehr nachweisbar & 220 & - \\
$\mathrm{Beginn}^{2+}$ der $\mathrm{Fe}^{2+}$-Reduktion & 150 & -185 \\
$\mathrm{Beginn}^{2}$ der $\mathrm{SO}_{4}{ }^{-}$-Reduktion und Sulfidbildung & -50 & -214 \\
$\mathrm{Beginn}^{2} \mathrm{CH}_{4}$-Bildung & -120 & -244 \\
$\mathrm{SO}_{4}{ }^{2}$ nicht $\mathrm{mehr}$ nachweisbar & -180 & - \\
\hline
\end{tabular}

Die Einflussgröße Anaerobie bzw. $\mathrm{O}_{2}$-Partialdruck spielt in ihrer räumlichen und zeitlichen Verteilung bei der Denitrifikation im Untergrund hydromorpher Böden eine wesentliche Rolle. Da die Sauerstoffdiffusion in wassergefüllten Poren etwa um das 10000-fache langsamer ist als in luftgefüllten, hat der Wassergehalt einen sehr großen Einfluss auf die Bildung anaerober Zonen.

Bei vollständiger Wassersättigung ist die $\mathrm{O}_{2}$-Nachlieferung aus der Atmosphäre somit extrem reduziert. Sofern die übrigen Voraussetzungen für die Denitrifikation gegeben sind, kann der Prozess unter Wassersättigung weitgehend ungehemmt ablaufen. 


\subsection{Quellen und Verfügbarkeit von Reduktionsmitteln}

Einer der wesentlichen Steuerparameter für die Denitrifikation im Boden ist die Verfügbarkeit von geeigneten Reduktionsmitteln (TIEDJE, 1988; KNOWLES, 1980). In Oberböden handelt es sich hierbei im wesentlichen um den organischen Kohlenstoff. Im Untergrund können auch reduzierte Eisen-Schwefel-Verbindungen (KöLLE et al., 1985; Rolland, 1995; Schmädecke, 1998; Lind \& Pedersen, 1976) eine bedeutende Rolle spielen. Organischer Kohlenstoff ist im Wurzelraum reichlich vorhanden. Nur ein geringer Teil davon ist für die Denitrifikation verfügbar (BEAUCHAMP et al., 1980). Unterhalb des Wurzelraums von Mineralböden kommt partikulärer organischer Kohlenstoff in Form von Torfbändern (SchUlte-KelLingHAUS, 1987) sowie als Braunkohlebeimengung in Geschieben (TEEPE, 1995), in fossilen Oberbodenhorizonten (WELL et al., 2001), oder in marinen und fluviatilen Sedimenten wie Marschen und Auenböden (MÜller-ThOMSEN, 1995; SCHMÄDECKE, 1998) vor. Der Beitrag dieser fossilen Kohlenstoff-Quellen zur Denitrifikation ist in einigen Arbeiten qualitativ belegt, aber es existieren noch kaum quantitative Untersuchungen $\mathrm{zu}$ ihrer Verfügbarkeit für die Denitrifikation.

Der Eintrag von gelöstem organischem Kohlenstoff $\left(\mathrm{C}_{\text {lös }}\right)$ mit dem Sickerwasser kann im Unterboden eine Substratquelle für die Denitrifizierer darstellen. Dieses ist als Folge der Aufbringung von organischen Düngern nachgewiesen (ARTIOLA, 1997; Bhogal und ShePherd, 1997; PAUL et al., 1998). SiEMENS (2003) hat eine geringe Verfügbarkeit von $\mathrm{C}_{\text {lös }}$ im Inkubationsversuch festgestellt. Ungeklärt ist somit, welcher Anteil des gelösten organischen Kohlenstoffs für die Denitrifikation verfügbar ist.

\subsection{Erschöpfbarkeit von Reduktionsmitteln durch Denitrifizierung von Nitra- teinträgen}

Für die Denitrifikation in tiefen Aquiferen ist schon länger bekannt, dass das gegebenenfalls vorhandene Denitrifikationspotential an fossile organische Substanz oder an reduzierte Eisen-Schwefel-Verbindungen gekoppelt ist und somit eine irreversibel erschöpfbare Größe darstellt (OBERMANN, 1989; KöLLE et al., 1985). Daraus wird gefolgert, dass in der Zukunft mit einer Erschöpfung des Denitrifikationspotentials und bei 
gleichbleibenden Nitrateinträgen mit einem Anstieg des Nitratgehalts im Förderwasser zu rechnen ist. Oberflächennahes Grundwasser hydromorpher Böden stellt den Grenzbereich zwischen Hydrogeologie und Bodenwissenschaften dar und wurde bisher unzureichend berücksichtigt.

Aus den letzten Jahren stammen einige Arbeiten, die auch für die ungesättigte Zone die Existenz eines irreversibel erschöpfbaren Denitrifikationspotentials zumindest qualitativ belegen. TEEPE (1995) fand in einer Pyrit- und braunkohlehaltigen Grundmoräne in ca. $3 \mathrm{~m}$ Tiefe eine deutlich reduktomorphe Denitrifikationszone. Die darüber liegende Zone ließ keine Denitrifikationsaktivität erkennen und wies sehr geringe Gehalte an Kohlenstoff und Schwefel auf. Dieser Befund wird als mögliche Folge der Erschöpfung einer ursprünglich vorhandenen Denitrifikationskapazität durch langjährige Nitrateinträge gedeutet.

SCHMÄDECKE (1998) beobachtete in Laborversuchen mit stark denitrifizierenden Bodensäulen aus dem Untergrund eines Auenbodens, dass deren permanente Durchwaschung mit Nitratlösung zu einem Verschwinden der visuell wahrnehmbaren Reduktionsmerkmale (Blaugraufärbung) im oberen Bereich der Bodensäulen führte. SOTOMAYOR \& RiCE (1996) untersuchten den Einfluss einer 50-jährigen Kultivierung eines Präriebodens auf die Denitrifikationsdynamik bis $7 \mathrm{~m}$ Tiefe. Im kultivierten Boden waren die Gehalte an löslichem Kohlenstoff und die Denitrifkationskapazität in 2 $4 \mathrm{~m}$ Tiefe im Vergleich zum nicht kultivierten Boden deutlich vermindert. Diesen Befund deuten sie als Erschöpfung der Denitrifikationskapazität infolge des Nitrateintrags mit dem Sickerwasser während der Kultivierungsperiode. RICHTER \& RICHTER (1991) sowie RoLLAND (1995) führten Laborversuche zur Nitratreduktion mit Gley- und Pseudogley-Böden durch, um die Erschöpfbarkeit des Denitrifikationspotentials zu untersuchen. Aus der kumulativen Nitratreduktion von inkubierten Bodenproben prognostiziert Rolland (1995) die Zeitspanne, in welcher in dem untersuchten Pseudogley-Boden der verfügbare organische Kohlenstoff durch Denitrifikation vollständig aufgebraucht wird. Für den Verlauf des Abbaus von reduzierten Schwefel-Eisen-Verbindungen gelang diese Prognose nicht, da die Bodenproben nicht anaerob gelagert wurden und somit ein Teil des reduzierten Schwefels durch Zutritt von Luftsauerstoff oxidiert wurde.

Eine detaillierte Untersuchung der chemolithoautotrophen Denitrifikation in Gegenwart von reduzierten Schwefel-Eisen-Verbindungen wurde im Aquifer des Fuhrberger Feldes (STADTWERKE HANNOVER) durchgeführt (KöLle et al., 1987; Frind et al., 1990; BÖTTCHER, 1992). KÖLLE et al. (1987) geben an, dass je nach mineralogisch- 
chemischer Zusammensetzung, Kristallqualität und Korngröße der Schwefel-Eisen Verbindungen nur ein Teil für die Denitrifikation verfügbar ist. Durch Inkubation von Aquifermaterial in wässriger Nitratlösung fanden sie heraus, dass durchschnittlich etwa $50 \%$ des Gesamtvorrats verfügbar waren.

\subsection{Auswirkung der Erschöpfung des Reduktionsmittelvorrats auf die Lachgas- bildung der Denitrifikation}

Der $\mathrm{N}_{2} \mathrm{O}$-Anteil der gasförmigen Denitrifikationsprodukte ist die entscheidende Kenngröße für den Beitrag des Nitratabbaus durch Denitrifikation zur Belastung der Atmosphäre. Der relative $\mathrm{N}_{2} \mathrm{O}$-Anteil an den gasförmigen $\mathrm{N}$-Verlusten des Bodens wird von vielen Steuergrößen beeinflusst. Wenn eine im Vergleich zum Nitratangebot mangelhafte Kohlenstoffverfügbarkeit auftritt, werden die Stickstoffoxide von den Denitrifikanten nur unvollständig genutzt. Stickstoffmonoxid (NO) wie auch Distickstoffoxid $\left(\mathrm{N}_{2} \mathrm{O}\right)$ treten dann als Endprodukt auf und entweichen in die Atmosphäre (Bouwman, 1990). Das Verhältnis von $\mathrm{N}_{2} \mathrm{O}$ zu N $\mathrm{N}_{2}$ sinkt mit zunehmender Feuchtigkeit des Bodens (ERIKSEN \& HolTAN-HARTWIG, 1993; BURESH et al., 1993) und steigt mit abnehmendem Gehalt an verfügbarem Kohlenstoff (WEIER et al., 1993 a). Die weiteren Faktoren, die das $\mathrm{N}_{2} \mathrm{O} / \mathrm{N}_{2}$-Verhältnis beeinflussen sind der $\mathrm{pH}$-Wert und die Temperatur. Die $\mathrm{N}_{2} \mathrm{O}$-Reduktase wird bei Temperaturen $<5^{\circ} \mathrm{C}$ und $\mathrm{pH}-$ Werten $<5$ überproportional verringert, so dass das $\mathrm{N}_{2} \mathrm{O} / \mathrm{N}_{2}-$ Verhältnis in sauren Böden und bei Temperaturen um den Gefrierpunkt deutlich ansteigt (WEIER \& Gilliam, 1986; Melin \& NommiK, 1983). Hohe Bodenwassergehalte und hohe Bodendichten fördern die Gesamt-Denitrifikation, resultieren aber in einem geringen $\mathrm{N}_{2} \mathrm{O}$-Anteil. Hohe Nitrat-Konzentrationen im Boden ergeben ebenfalls einen erhöhten $\mathrm{N}_{2} \mathrm{O}$-Anteil.

Aus den genannten Beziehungen geht hervor, dass der $\mathrm{N}_{2} \mathrm{O}$-Anteil im Denitrifikationsgas steigt, wenn die Verfügbarkeit von Reduktionsmitteln in Relation zur Nitratverfügbarkeit abnimmt. Daraus ist zu folgern, dass eine zunehmende Erschöpfung von Reduktionsmitteln im Boden bei gleichbleibendem Nitrateintrag zu einem steigenden $\mathrm{N}_{2} \mathrm{O}$-Anteil führen muss. 


\section{Zielsetzung}

Im Rahmen dieser Arbeit sollen folgende Aspekte bearbeitet werden:

- Vorkommen, Variabilität und regionale Verbreitung von Reduktionsmitteln in hydromorphen Böden.

- Gesamtvorräte, Zusammensetzung (C-, S- und $\mathrm{Fe}^{2+}$-Spezies) und die Verfügbarkeit der Reduktionsmittel für die Denitrifikation.

Die Identifizierung und Quantifizierung verschiedener Formen der Reduktionsmittel ist wichtig, um die Auswirkung der Reduktionsmittel-Gehalte auf Nitratverluste durch Denitrifikation in ausgewählten Untersuchungsstandorten (Gleye, Marschen- und Auenböden) zu erhalten.

Die Erschöpfbarkeit des Denitrifikationspotentials in der gesättigten Zone verschiedener hydromorpher Böden in Norddeutschland (Gleye, Marschen- und Auenböden) soll bestimmt werden, um dadurch die langfristige Konsequenz von Nitratausträgen aus der Landwirtschaft im Hinblick auf die Grundwasserqualität zu prognostizieren. Die Untersuchungen sollen im Bereich des oberflächennahen Grundwassers durchgeführt werden, d.h. in einer Tiefenzone, die den Schwankungsbereich des Grundwasserspiegels sowie die ersten 2 bis $3 \mathrm{~m}$ der gesättigten Zone umfasst.

Die Abbaurate der Reduktionsmittel soll in Laborversuchen direkt bestimmt werden, um daraus quantitative Aussagen für die bereits erfolgte und bei gleichbleibenden Nitratausträgen in Zukunft erwartete Erschöpfung des Denitrifikationspotentials abzuleiten.

Darüber hinaus ist der Einfluss der langfristigen Erschöpfung der Reduktionsmittel auf den Anteil klimarelevanter Gase in den gasförmigen Denitrifikationsprodukten zu untersuchen. 


\section{Material und Methoden}

\subsection{Untersuchungsgebiete}

Die ausgewählten Untersuchungsgebiete (Tab. 2) sind zum Teil Flächen aus dem Bodendauerbeobachtungsprogramm des Niedersächsischen Landesamtes für Bodenforschung (NLfB) (Vechtel, Dinklage, Aher Kämpe, Bühren, Schladen, Neuhäuser- Felde). Die Untersuchungsflächen der Universität Göttingen (Markhausen und Freistatt) sind Versuchsflächen, wo u. a. die Auswirkung des N-Einsatzes in Gebieten mit hoher Viehdichte auf die Qualität des Sickerwassers untersucht wird. Eine Fläche des Versuchsguts der Universität Göttingen (Reinshof) gehört ebenso zum ausgewählten Untersuchungsgebiet.

Diese landwirtschaftlich genutzten Flächen schienen geeignet, da hier zuverlässige Bewirtschaftungsangaben vorlagen.

Auf der Fläche Fuhrberg führt die Universität Hannover seit langem Untersuchungen zur Auswirkung der Bodennutzung auf die Grundwasserqualität durch.

In Abb. 3 ist die Karte der Grundwasserflurabstände in Niedersachsen mit der Lage der untersuchten Böden eingezeichnet.

Es wurden Böden ausgewählt, die in unterschiedlichem Maße fossile Reduktionsmittel im Untergrund aufweisen und die für die Trinkwassergewinnung von Bedeutung sind. In Niedersachsen werden etwa $87 \%$ des Trinkwasserbedarfs über zentrale Wassergewinnungsanlagen aus den Grund- und Quellenwasservorkommen gedeckt. Eine besondere Bedeutung kommt hierbei den ergiebigen Grundwasservorkommen der Geest als Lockersedimentgebiet mit verhältnismäßig hohen Niederschlägen zu (SEEDORF \& MEYER, 1992).

Die Flächen der Bodendauerbeobachtung repräsentieren in erster Linie die Verbreitung der verschiedenen Boden- und Nutzungstypen in Niedersachsen. Diese Flächen sind z.T. Gegenstand anthropogener Bodenbelastungen durch die Nähe zu industriellen Emissionsgebieten oder durch hohen Viehbesatz der bewirtschaftenden Betriebe. Zum anderen Teil werden auch relativ unbelastete Böden untersucht, die für die Trinkwassergewinnung sowie für den Naturschutz eine besondere Rolle spielen.

Ein Teil der Untersuchungsgebiete liegt nach LIEDTKE (1984) in der Altmoränenlandschaft des "Norddeutschen Tieflandes" die sich größtenteils aus Geschiebedecksanden 


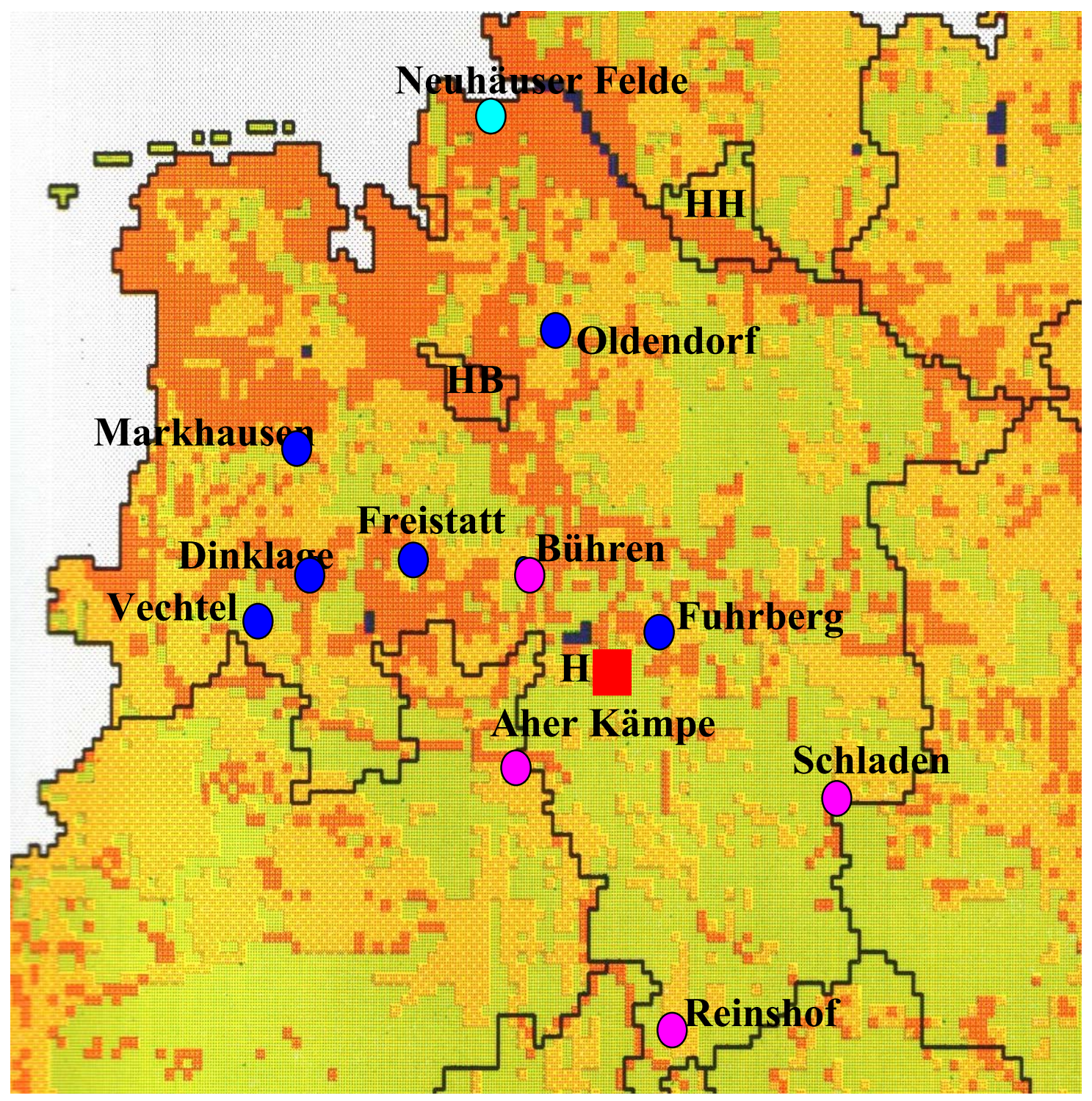

Abb. 3: Karte der Grundwasserflurabstände in Niedersachsen mit der Lage der untersuchten Böden (nach WENDLAND et al., 1993)

Gelb : $<1 \mathrm{~m}$

Orange: $1-2 \mathrm{~m}$

Grün : > $2 \mathrm{~m}$

○ Auen-Böden

- gleyartige Sand-Böden

○ Marsch-Böden 
und Geschiebelehmen des Pleistozäns (ScHEFFER \& ScHACHTSCHABEL, 1992) entwikkelt hat. Bei fehlendem Geschiebelehm haben sich in den Böden des Flachlandes in den Sanden Braunerden und Podsole entwickelt (KUNTZE et al., 1994). Die niedersächsischen Geestlandschaften werden üblicherweise in die "Hohe Geest" (Endmoränenzüge), die "Geestplatten" und die "Niedere Geest" untergliedert (SEedorf \& MEYER, 1992). Die Bodenregion der Geest ist eine Altmoränenlandschaft und wurde von den Gletschern der vorletzten Eiszeit geprägt. Die Landschaft gliedert sich in Grundmoränen, Endmoränen, Sander und Urstromtäler. Für die Bodenentwicklung und -eigenschaften der Geest ist von Bedeutung, dass Sickerwasserbildung, Vernässung, Torfbildung, Versauerung und Stoffverlagerung in Richtung Osten abnehmen. Die Folge ist eine stärkere Verbreitung von Stauwasserböden, Mooren und Podsolen im Westen sowie Braunerden im Osten. In den Niederungen sind geringe Grundwasserflurabstände typisch. Neben der Vermoorung hat dies auch zur Vergleyung der Mineralböden geführt. Die Geest ist als Folge ihrer Entstehungsgeschichte eine Landschaft mit vergleichsweise nährstoffarmen Böden. Intensive Nutzung führt heute auf diesen sorptionsschwachen Böden verbreitet zu Nitrataustrag in das Grundwasser.

Die typischen Marschen an der Küste mit ihren charakteristischen tonreichen Böden, die sich durch die Auflandung von Wattsedimenten gebildet haben, werden überwiegend als Grünland bewirtschaftet. Die Bildung dieser Großlandschaft beruht im wesentlichen auf dem langsamen Meeresspiegelanstieg in den letzten 10.000 Jahren. Dabei wurden die vorwiegend feinkörnigen Sedimente des Meeres im Gezeiteneinflussbereich sedimentiert. Die Böden der Marschen sind durch die relativ gleichkörnigen Sedimente und den Grundwassereinfluss geprägt. In Abhängigkeit von den Grundwasserständen, den Sedimenteigenschaften und dem Alter der Marschen sind die Böden unterschiedlich entsalzt und entkalkt. In weiter fortgeschrittenen Stadien kommt es bei starker Versauerung zu Schwefelanreicherungen (Maibolt).

In den Flussauen sind in der Regel nach der Sedimentausbildung drei Komplexe (ältester, älterer und jüngerer Auenlehm) zu unterscheiden. Die ältesten Auenlehme sind häufig sehr tonig und tragen z.T. einen schwarzen, reliktischen Boden. Die älteren Auenlehme sind im Oberboden entkalkt, wogegen jüngeren Auenlehme, zum großen Teil infolge der mittelalterlichen und neuzeitlichen Bodenerosion im Oberlauf gebildet, noch nicht entkalkt sind. Die Auenböden sind aufgrund ihrer hohen biologischen Aktivität homogen (braun) gefärbt. Bei gestörter biologischer Aktivität ist die Sedimentschichtung erhalten. Bei steigendem Grundwassereinfluss bildeten sich Gleye. 
Das Land Niedersachsen gliedert sich geographisch von Nord nach Süd in das nordwestdeutschen Flachland, in die Bördelandschaften, das südniedersächsische Bergund Hügelland und den Harz als Mittelgebirge. Das niedersächsische Klima unterliegt einem stark maritimen Einfluss mit hohem Niederschlag im Küstengebiet.

Die 11 ausgewählten Untersuchungs-Flächen decken weitgehend das lokale Spektrum der grundwasserbeeinflussten Mineralböden (Gleye, Marschböden, Auenböden) ab.

Tab. 2: Untersuchungsgebiet

\begin{tabular}{llllll}
\hline Standort & Bodentyp & Bodenart & Nutzung & $\begin{array}{l}\text { GW-Flur- } \\
\text { abstand } \\
\text { (cm) }\end{array}$ & $\begin{array}{l}\text { Untersuchtes } \\
\text { Tiefen-Intervall } \\
\text { (cm) }\end{array}$ \\
\hline Aher-Kämpe & Auen-Gley & Tu4-Tu3 & Grünland & 160 & $140-240$ \\
Bühren & Auen-Pseudogley & Ut4-Tu3 & Acker & 250 & $200-320$ \\
Reinshof & Auen-Gley & T1-Ls3 & Acker & 130 & $110-190$ \\
Schladen & Auen-Gley & Ls4-U14 & Acker & 270 & $160-340$ \\
Neuhäuser- & Seemarsch & Sl3-Tu3 & Acker & 250 & $100-320$ \\
Felde & & & & & $100-400$ \\
Fuhrberg & Podsol-Gley & S & Acker & 140 & $140-300$ \\
Freistatt & Podsol-Gley & S12 & Grünland & 110 & $150-310$ \\
Markhausen & Tiefenumbruchboden & Su2-S & Acker & 150 & \\
& aus Hochmoor & & & & $140-400$ \\
Dinklage & Gley-Podsol & Su3-S & Acker & 145 & $100-140$ \\
Oldendorf & Gley-Podsol & Su3-S & Grünland & 120 & $80-140$ \\
Vechtel & Gley-Podsol & S & Acker & 95 & \\
\hline GW=Grundwasser & & & & \\
\hline
\end{tabular}

$\mathrm{GW}=$ Grundwasser

\subsection{Probenahme und Probenaufbereitung}

Aus dem Untergrund der Untersuchungsflächen wurden im oberflächennahen Grundwasser sowie im Kapillarsaum in Intervallen von 10-20 cm Proben entnommen (vgl. Tab. 2), wobei sich der beprobte Bereich zwischen mindesten $1 \mathrm{~m}$ und höchstens $4 \mathrm{~m}$ unter der Geländeoberkante befand. Die Beprobung der gesättigten Zone erfolgte in bindigen Böden mit dem Edelmannbohrer (Bohrkopfdurchmesser $5 \mathrm{~cm}$ ), in Sandböden mit Schlammbüchse (Durchmesser $10 \mathrm{~cm}$, Firma Eijkelkamp) und Schutzverrohrung. 
Das Bodenmaterial wurde im Gelände in Plastikbeutel verpackt und in der Kühlkammer für weitere Untersuchungen bei $4-5^{\circ} \mathrm{C}$ gelagert. Der bei Göttingen gelegene Auen-Gley (Reinshof) wurde intensiver beprobt, weil hier verschiedene methodische Voruntersuchungen durchgeführt wurden.

\subsection{Laborexperimente}

\subsubsection{Anaerobe Langzeitinkubationen zur Erschöpfung der Denitrifikation}

$25 \mathrm{~g}$ frischer Bodenproben wurden in Rollrandflaschen $(115 \mathrm{ml})$ nach Zugabe von 50 $\mathrm{ml}$ wässrigen $\mathrm{K}^{15} \mathrm{NO}_{3}$-Lösung mit einer Konzentration von $100 \mathrm{mg}^{-1} \mathrm{NO}_{3}-\mathrm{N}$ unter anaeroben Bedingungen bei $25^{\circ} \mathrm{C}$ inkubiert. Hierbei wurde durch den Einsatz des ${ }^{15} \mathrm{~N}$ Isotops als Tracer das Verhalten des Nitrats im Boden verfolgt um somit die Prozesse

des N-Umsatzes zu erfassen. Die ${ }^{15} \mathrm{~N}$-Tracertechnik stellt eine Methode dar, welche grundsätzlich geeignet ist, auch geringe Stickstofffreisetzungen durch massenspektrometrische Messungen zu ermitteln. Durch die Kombination massenspektrometrischer Messungen und gaschromatographischer Verfahren, ist eine Differenzierung der mikrobiologischen Prozesse, welche an der gasförmigen N-Freisetzung beteiligt sind, möglich. Die ${ }^{15} \mathrm{~N}$-Messungen erfolgten aus Kostengründen nicht bei allen Proben. Die Flaschen wurden mit einem gasdichten Septum verschlossen, mehrfach evakuiert und mit $\mathrm{N}_{2}$ wieder auf Atmosphärendruck gebracht. Zur Bestimmung des Temperatureinflusses wurde ein Teil der Proben bei $10^{\circ} \mathrm{C}$ inkubiert. Um den Nitratverbrauch zu ermitteln wurde in 3 bis 7 Intervallen beprobt, wobei die Länge des Intervalls mit der Versuchsdauer zunahm (Tab. 3). Am Ende jedes Intervalls wurde die überstehende Lösung von den Proben mittels einer Dispensette abgesaugt und analysiert. Die Differenz zwischen vorgelegter und wiedergefundener Nitratmenge wird der Denitrifikation zugerechnet. Die Lösung wurde nach jeder Messung erneuert. 
Tab. 3: Zeitnitervalle der anaeroben Inkubation

\section{Inkubationsintervalle in Tagen}

\begin{tabular}{llllllll}
\hline Fläche & 1 & 2 & 3 & 4 & 5 & 6 & 7 \\
\hline Reinshof & 60 & 50 & 50 & 132 & 161 & 178 & 214 \\
Oldendorf & 50 & 50 & 133 & 161 & 178 & 214 & \\
Vechtel & 17 & 50 & 133 & 161 & 181 & & \\
Aher Kämpe, Dinklage, Mark- & 42 & 74 & 127 & 149 & 211 & & \\
hausen, Schladen & & & & & & & \\
Bühren, Neuhäuser-Felde & 42 & 74 & 127 & & & & \\
\hline
\end{tabular}

Zur direkten Messung der Denitrifikation wurde der ${ }^{15} \mathrm{~N}$-Gehalt in der Gasphase (Headspace) gemessen. Dies erfolgte am Ende jedes Inkubationsintervalls. Das ursprüngliche Ziel war es, neben der Quantifizierung der Gesamtdenitrifikation $\left({ }^{15}\left(\mathrm{~N}_{2}+\mathrm{N}_{2} \mathrm{O}\right)\right)$ auch den $\mathrm{N}_{2} \mathrm{O}$-Anteil im Denitrifikationsgas $\left({ }^{15} \mathrm{~N}_{2} \mathrm{O}\right)$ zu bestimmen. Da der $\mathrm{N}_{2} \mathrm{O}$-Anteil in geschlossenen Systemen mit fortschreitendem N-Umsatz durch Reduktion $\mathrm{zu} \mathrm{N} \mathrm{N}_{2}$ abnimmt (FIRESTONE et al., 1979) sollte die Gasanreicherung in den Flaschen nur kurzzeitig erfolgen. Deshalb wurden die Flaschen am Ende der mehrwöchigen Inkubationsintervalle nach $\mathrm{N}_{2} \mathrm{O}$ und $\mathrm{CO}_{2}$-Messung wie zuvor beschrieben evakuiert und mit $\mathrm{N}_{2}$ gespült. Anschließend wurden sie für 1, 5 oder 24 Stunden wieder inkubiert, um die akkumulierten Gase zu entfernen. Die Inkubationszeit wurde variiert, um den denitrifikativen N-Umsatz für jede Probe in einem engem Schwankungsbereich zu halten. Proben mit hoher Aktivitäten wurden nur kurz ( $1 \mathrm{~h})$ die mit geringster Aktivität am längsten (24 h) inkubiert. Die Größenordnung der Denitrifikationsraten war aus Parallelversuchen (WELL, 2001) bereits bekannt. Am Ende der Inkubationszeit wurde ein Aliquot von 15 $\mathrm{ml}$ Gas mit einer Plastikspritze in evakuierte 12-ml-Septumflaschen injiziert und so bis zur Isotopenanalyse aufbewahrt.

Zur Bestimmung der Akkumulation von $\mathrm{N}_{2} \mathrm{O}$ und $\mathrm{CO}_{2}$ in den Langzeit-Inkubationsversuchen wurde das Gasvolumen in den Flaschen mit dem Bodenmaterial und der zugegebenen $\mathrm{K}^{15} \mathrm{NO}_{3}$-Lösung jeweils vor dem Lösungsaustausch analysiert. 


\subsubsection{Sulfat-Produktion}

Um die Sulfat-Produktion an den Langzeit-Inkubationsversuchen zu quantifizieren, wurden die Sulfat-Gehalte der Lösungen gemessen. Es wurden die gleichen Intervallen wie für die Nitrat-Reduktion (s. Tab. 3) verwendet .

\subsubsection{Aerobe Inkubation zur Bestimmung der mikrobiellen Oxidation des Sulfids}

Da im Untergrund außer organischem Kohlenstoff auch reduzierte Eisen-SchwefelVerbindungen (KöLle et al., 1985; Rolland, 1995; SCHMÄDECKE, 1998; LIND \& Pedersen, 1976) eine bedeutende Rolle bei der Denitrifikation spielen, wurde zur Charakterisierung des verfügbaren Sulfid-Schwefels neben der Sulfid-Messung (vgl. Kap. 4.4.3.) und der anaeroben Sulfatproduktion (vgl. 4.4.3.1 Sulfat-Produktion) auch die aerobe Sulfatproduktion gemessen. Dazu wurde eine zweite Teilprobe in gasdurchlässigen PE-Beuteln bei $25^{\circ} \mathrm{C}$ bebrütet. Nach Bebrütungsbeginn wurde nach 6 bis 7 Intervallen (Tab. 4) auf Sulfat untersucht.

Tab. 4: Zeitintervalle der aeroben Inkubation

\begin{tabular}{llllllll}
\hline & \multicolumn{7}{c}{ Inkubationsintervalle in Tagen } \\
\hline Fläche & 1 & 2 & 3 & 4 & 5 & 6 & 7 \\
\hline Oldendorf & 13 & 70 & 159 & 278 & 390 & 544 & 722 \\
Dinklage & 20 & 76 & 233 & 345 & 526 & 740 & \\
Aher Kämpe, & 9 & 37 & 121 & 232 & 386 & 569 & \\
Markhausen & 12 & 55 & 138 & 251 & 406 & 588 & \\
Schladen & 7 & 49 & 136 & 245 & 400 & 584 & \\
Neuhäuser-Felde & 11 & 46 & 98 & 214 & 368 & 548 & \\
\hline
\end{tabular}

\subsection{Analytik}

\subsubsection{Stickstoff}

Nitrat- und Ammonium-Bestimmung erfolgte über das Dampfdestillationsverfahren (BREMNER \& KEENEY, 1965). Für die Analyse von Bodenparametern wurden $200 \mathrm{~g}$ 
feldfrisches Bodenmaterial mit $250 \mathrm{ml} 0,02 \mathrm{n} \mathrm{CaCl}_{2}$-Lösung versetzt, etwa $1 \mathrm{~h}$ geschüttelt und abfiltriert. Zur Bestimmung von Ammonium wurden 5 bis $30 \mathrm{ml}$ des filtrierten Extrakts mit Magnesiumoxid $(\mathrm{MgO})$ versetzt und erhitzt um das Ammonium in Ammoniak $\left(\mathrm{NH}_{3}\right)$ zu überführen. Das entstandene Ammoniak wird durch Dampfdestillation in eine 2-\%ige Borsäure-Lösung $\left(\mathrm{H}_{3} \mathrm{BO}_{3}\right)$ überführt. Durch Rücktitration mit 0,01 $\mathrm{n} \mathrm{HCl}$ wurde die Ammoniummenge bestimmt.

Zur Ermittlung des Nitrat-Gehaltes wurde der Extrakt anschließend mit 0,5 g DevardaLegierung (50\% Cu, 45\% Al und 5\% Zn) versetzt um Nitrat zu Ammonium zu reduzieren. Die Destillation wurde analog zum Ammonium wiederholt. Für die Analyse der Versuchslösung der Dauerinkubationsversuche wurden 5-30 ml des klaren Überstands verwendet und wie bereits beschrieben auf Nitrat analysiert. Bei der Messung wurde jeweils ein Blindwert durch Destillation von $20 \mathrm{ml}$ reiner $0,02 \mathrm{n} \mathrm{CaCl}_{2}$-Lösung ermittelt.

\subsubsection{Kohlenstoff}

\subsubsection{Gesamt-Kohlenstoff $\left(C_{t}\right)$ und Gesamt-Stickstoff $\left(N_{t}\right)$}

Gesamtgehalte an Kohlenstoff und Stickstoff wurden mit Hilfe der Elementaranalyse (Carlo-Erba, NA 1400) nach DuMAs ermittelt. Hierzu wurden die Proben bei $105^{\circ} \mathrm{C}$ getrocknet und in einer Kugelmühle pulverisiert. 10 - $20 \mathrm{mg}$ der Proben wurden in Zinnkapseln eingewogen und in einem Quarzrohr in reinem Sauerstoff (99,998 \%) bei $1050^{\circ} \mathrm{C}$ zu CO $\mathrm{CO}_{2}$ verbrannt. Bei der Verbrennung dienen $\mathrm{Cr}_{2} \mathrm{O}_{3}$ und Silberwolle als ClFilter und $\mathrm{O}_{2}$-Puffer. Die Verbrennungsgase werden mit dem Trägergas Helium weitergeleitet. Während Sauerstoff eliminiert wird, werden Stickstoffoxide in einem mit Kupfer gefüllten Quarzrohr bei $650^{\circ} \mathrm{C}$ reduziert. Nach dem Passieren der Wasser- und $\mathrm{CO}_{2}$ Falle (Glasrohr, zu 1/3 gefüllt mit $\mathrm{Mg}\left(\mathrm{ClO}_{4}\right)_{2}$, zu 2/3 gefüllt mit Ascarite) erfolgt die Auftrennung des Gasgemisches in der Trennsäule. Anschließend wird der $\mathrm{CO}_{2}$ - und der $\mathrm{N}_{2}$-Peak in einem Wärmeleitfähigkeits-Detektor (WLD) registriert. Die Messsignale werden über einen Integrator ausgewertet. 


\subsubsection{Gesamtorganischer Kohlenstoff $\left(C_{\text {org }}\right)$}

Organischer Kohlenstoff $\left(\mathrm{C}_{\text {org }}\right)$ wurde als Differenz zwischen dem am Elementaranalysator gemessenen Gesamt-Kohlenstoff und dem Calciumkarbonat $\left(\mathrm{CaCO}_{3}\right)$-Anteil (s. Kap. 4.4.2.5) der getrockneten Proben bestimmt.

\subsubsection{Gelöster organischer Kohlenstoff $\left(\mathrm{C}_{\text {lös }}\right)$}

Die Analyse für den gelösten organischen Kohlenstoff $\left(\mathrm{C}_{\text {lös }}\right)$ wurde mit dem CAnalysator der Firma MAIHAK (Hamburg) durchgeführt. Bei $800^{\circ} \mathrm{C}$ erfolgt eine vollständige Verbrennung des im Extrakt gelösten Kohlenstoffes. Das Verbrennungsgas wird danach einem UV-Detektor zugeführt, der durch die Messung des $\mathrm{CO}_{2}$ direkt die Kohlenstoff-Konzentration der Probenlösung (mg/l) errechnet wird. Vor der Analyse wird $\mathrm{CO}_{2}$ durch Ansäuern der Proben mit 0,01 n $\mathrm{HCl}$ auf $\mathrm{pH} 3,5$ ausgetrieben und wird somit bei der Analyse nicht erfasst.

Die Behandlung der Proben sollte sicher stellen, dass möglichst nur die zum Zeitpunkt der Probenahme gelöste Fraktion des organischen Kohlenstoffes gemessen wird. Da sich nach einer Konservierung durch Trocknung deutlich mehr Kohlenstoff extrahieren lässt (BECKER et al. 1988 a ), erfolgte die $\mathrm{C}_{\text {lös }}$-Bestimmung ausschließlich an feldfrischen Bodenproben.

\subsubsection{Heißwasserlöslicher Kohlenstoff $\left(C_{h w l}\right)$}

Die Bestimmung des heißwasserlöslichem Kohlenstoff erfolgte in Anlehnung an BEHM (1988). $10 \mathrm{~g}$ feingemahlener Boden wurden mit $50 \mathrm{ml}$ destilliertem Wasser versetzt und 1 Stunde lang im Trockenschrank bei $110^{\circ} \mathrm{C}$ gekocht. Nach dem Abkühlen wurden 3 Tropfen 5 n Calciumchloridlösung zugesetzt und die Suspension wurde mit Blaubandfilter (5893, Fa. SCHLEICHER und SCHUELL) abfiltriert. Danach erfolgte die Bestimmung von $\mathrm{C}_{\mathrm{hwl}}$ des Filtrats am C-Analysator der Firma MAIHAK, HAMBURG (vgl. Kap. 4.4.2.1). 


\subsubsection{Carbonat-Kohlenstoff}

Die Carbonatgehalte der Böden wurden gasvolumetrisch unter Berücksichtigung von Luftdruck und Temperatur nach dem ScheIBLER-Verfahren (KRETSCHMAR, 1991) bestimmt. Hierzu wurden die Proben bei $105^{\circ} \mathrm{C}$ getrocknet und in der Kugelmühle pulverisiert. Es wurden jeweils $2 \mathrm{~g}$ Boden eingewogen und in der SCHEIBLER-Apparatur mit ca. $20 \mathrm{ml} \mathrm{1:3} \mathrm{verdünnter}(10 \%)$ Salzsäure $(\mathrm{HCl})$ versetzt. Die sich dabei entwickelnde Kohlendioxid-Menge ist proportional zu der in der Bodenprobe enthaltenen Menge an $\mathrm{CaCO}_{3}$. Die Eichung der Apparatur erfolgte, in dem zu Beginn einer Versuchsreihe und nach jeder 4. Probe einen Blindwert mit $200 \mathrm{mg}$ trockenem, chemisch reinem $\mathrm{CaCO}_{3}$ mit analysiert wurde.

\subsubsection{Schwefel}

\subsubsection{Gesamt- und Sulfid-Schwefel}

Bei der Analyse von Sulfiden in reduzierten Sedimenten ist darauf zu achten, dass durch Kontakt mit atmosphärischem Sauerstoff bei der Entnahme, Lagerung und Aufbereitung der Proben eine Teil-Oxidation zu Sulfat erfolgen kann, die somit zur einer Unterschätzung des Sulfid-Gehalts führt (z.B. Rolland, 1995). Deshalb wurden zunächst verschiedene Techniken geprüft, um die Sulfidoxidation zu unterdrücken. Es wurden vier verschiedene Varianten getestet. Bei der ersten Variante wurden die Proben direkt nach der Entnahme im Feld in Flaschen mit Septumverschlüssen gefüllt und etwa 30 min. lang mit Stickstoff gespült, der Verschluss wurde zusätzlich mit Silikon abgedichtet. Anschließend wurden die Proben im Gefrier-Trockner unter Vakuum getrocknet. Nach etwa einer Woche Trocknung wurden sie in der Kugelmühle gemahlen und danach am Schwefelanalysator gemessen. Bei der zweiten Variante erfolgte zusätzlich zu den Maßnahmen der ersten Variante eine Begasung mit Argon im Gefrier-Trockner. In der dritten Variante wurde ohne vorherige $\mathrm{N}_{2}$-Behandlung im Gefrier-Trockner unter Vakuum getrocknet. Bei der vierten Variante wurden Parallelproben ohne vorherige $\mathrm{N}_{2}$ Spülung im Umlufttrockenschrank bei drei Temperaturen $\left(35^{\circ} \mathrm{C}, 50^{\circ} \mathrm{C}\right.$ und $\left.105^{\circ} \mathrm{C}\right)$ getrocknet. Die Ergebnisse zeigten, dass auch in den Varianten mit $\mathrm{O}_{2}$-freie Lagerung (1. und 2. Variante) eine Sulfidoxidation nicht vollständig unterdrückt werden konnte. Deshalb wurden diese Behandlungen in den Hauptversuchen nicht mehr durchgeführt. 
Stattdessen wurde die Sulfidoxidation bei der Probenlagerung aus der Differenz zwischen den Sulfatgehalten frischer und gelagerter Proben (s. Kap. 4.4.3.2) bestimmt.

Für die Gesamt- und Sulfidschwefelmessung am Kohlenstoff/Schwefel Analysator (E1tra CS 500) wurden $500 \mathrm{mg}$ der getrockneten und in der Kugelmühle fein gemahlenen $(<63 \mu \mathrm{m} \varnothing)$ Bodenproben verwendet (BRUMSACK, 1981). Sulfid-Schwefel wurde bei $750^{\circ} \mathrm{C}$ im $\mathrm{O}_{2}$-Strom verbrannt und als $\mathrm{SO}_{2}$ gemessen. Die Absorption dieses Gases wird im Infrarot- Bereich gemessen. Um den Sulfat-Schwefel zu bestimmen wurden die Proben nach der Sulfid-Schwefelbestimmung noch einmal bei $1400^{\circ} \mathrm{C}$ verbrannt. Gesamtschwefel wurde aus der Summe von Sulfid-Schwefel und Sulfat-Schwefel errechnet. Zum Sulfid-Schwefel wurde die aus den Sulfat-Gehalten frischer und gelagerter Proben berechnete Sulfid-Oxidation (s.o.) hinzu addiert.

\subsubsection{Extrahierbarer Sulfat-Schwefel}

Für die Sulfatextraktion wurden 200 g Boden je Tiefe mit $250 \mathrm{ml}$ 0,02 n CaCl 2 -Lösung versetzt, etwa 1 h horizontal geschüttelt und abfiltriert. Die Sulfatanalyse an der Bodensuspension erfolgte durch Rücktitration von $\mathrm{Ba}^{++}$nach Zugabe von $\mathrm{BaCl}_{2}$. Zunächst wird der Extrakt durch eine mit $\mathrm{H}^{+}$-Ionen belegte Kationenaustauschersäule perkoliert, um alle Kationen zu entfernen. Danach werden dem Perkolat $5 \mathrm{ml} \mathrm{BaCl}_{2}$ zugegeben. Es bildet sich $\mathrm{BaSO}_{4}$, das als schwerlöslicher weißer Niederschlag ausfällt. Durch Titration

der freien $\mathrm{Ba}^{2+}$-Ionen kann die Menge an Sulfationen, die als $\mathrm{BaSO}_{4}$ ausgefallen sind, errechnet werden. Ein Ziel der Inkubationsversuche war es, festzustellen wie die SulfidVerfügbarkeit das Denitrifikationspotential beeinflußt. Um die Sulfidoxidation während der Lagerung und Aufbereitung der Bodenproben zu bestimmen, wurden zunächst die Sulfat-Gehalte an feldfrischen Böden direkt nach der Probenahme ermittelt. Anschließend wurde das restliche Bodenmaterial für die weitere Beprobung in Plastiktüten bei $25^{\circ} \mathrm{C}$ gelagert. Bei der späteren Messung des Gehalts an Sulfid-Schwefel (vgl. Kap. 4.4.3) wurde die Differenz zwischen den Sulfatgehalten der gelagerten und der frischen Proben verwendet, um die Sulfidoxidation während der Probenlagerung abzuschätzen.

\subsubsection{Gelöstes Eisen ( $\left.\mathrm{Fe}_{\text {lös }}\right)$}

Die Bestimmung von gelöstem Eisen erfolgte mit Hilfe eines Atom- AdsorptionsSpektrometers (AAS) der Firma VARIAN AA-775. Hierzu wurde Von dem Probenmate- 
rial 200 g geländefrischer Boden je Tiefe mit $250 \mathrm{ml}$ 0,02 n CaCl${ }_{2}$-Lösung versetzt, etwa $1 \mathrm{~h}$ geschüttelt und filtriert. Die AAS-Messung erfolgte am klaren Extrakt.

\subsubsection{Bodenwassergehalt}

Die Bodenwassergehalte wurden gravimetrisch durch Trocknung des Bodens bis zur Gewichtskonstanz bei $105^{\circ} \mathrm{C}$ ermittelt. Der Gewichtsverlust bezogen auf die Trockensubstanz ergibt den Wassergehalt in Gewichts- \%.

\subsubsection{Korngrössenanalyse}

Um Aussagen über die Korngrößenzusammensetzung der unterschiedlichen Standorte machen zu können, wurde eine Korngrößenanalyse nach einem kombinierten Sieb- und Sedimentationsverfahren durchgeführt. Gesiebt wurde mit Prüfsieben nach DIN. Hierzu wurden $20 \mathrm{~g}$ des bei $105^{\circ} \mathrm{C}$ zur Gewichtskonstanz getrockneten Bodenmaterials eingewogen und mit 200-300 ml Wasser versetzt. Zur Dispergierung wird $1 \mathrm{~g}$ in einer 1,25prozentigen $\mathrm{NaOH}-L o ̈ s u n g ~ g e l o ̈ s t e s ~ N a t r i u m-D i t h i o n i t$, sowie $30 \mathrm{ml}$ 0,3 M Na-Citrat zugegeben. Die Proben wurden zwei Tage lang bei $25^{\circ} \mathrm{C}$ mit zwischenzeitlich mehrmaligem Aufschütteln aufbewahrt. Bei kalkhaltigen Proben wird Carbonat zuvor mittels 10-prozentiger Salzsäure ausgetrieben, wobei auf eine Mineralauflösung bzw. -anlösung bei $\mathrm{pH}$-Werten unter 3,5 zu achten ist. Im Anschluss an diese Reaktionszeit beginnt die Nasssiebung der Proben. Hiermit werden die Fraktionen Bodenskelett $(\varnothing>2 \mathrm{~mm})$, Grob-, Mittel- und Feinsand $(\varnothing=2000-63 \mu \mathrm{m})$, sowie Grobschluff $(20-63 \mu \mathrm{m})$ erfasst. Nach der Nasssiebung erfolgt eine 3-minütige Ultraschallbehandlung des restlichen Probenmaterials, um nochmals die Dispergierung der Bodenteilchen sicherzustellen. Das so behandelte Bodenmaterial wird in eine ATTERBERG-Apparatur (ATTERBERG, 1912) mit einer Sedimentationshöhe von $30 \mathrm{~cm}$ überführt. Die Trennung der Korngrössenfraktionen vollzieht sich hier nach dem STOKES'schen Widerstandsgesetz entsprechend ihrer spezifischen Fallzeit. Der trübe Überstand wird jeweils entnommen und gesammelt. Die Abtrennung einer Fraktion ist vollständig, wenn der Überstand nach Ablauf der spezifischen Absinkzeit der nächst gröberen Fraktion klar ist. Hierzu muss meist mehrfach aufgeschüttelt und entnommen werden. Die einzelnen Fraktionen werden getrocknet, gewogen und ihr Gewichtsanteil an der Gesamtprobe berechnet. Anhand dieser Methode werden die Fraktionen Mittel-, Feinschluff und Ton ermittelt. 


\subsection{7 pH-Wert}

Die Bestimmung des $\mathrm{pH}-$ Werts der Bodenproben erfolgten elektrometrisch an Bodensuspensionen mittels pH-Meter (Typ 632 der Firma Metrohm). Dazu wurden 10 g luftgetrockneter Boden mit $20 \mathrm{ml}$ einer 0,02 $\mathrm{n} \mathrm{CaCL}_{2}$-Lösung versetzt und einige Male umgerührt. Nach 20 Minuten wurde der pH-Wert der Bodensuspension mit der Glaselektrode des $\mathrm{pH}$-Meters bestimmt.

\subsubsection{Gaschromatographie}

Die $\mathrm{CO}_{2}$-Konzentrationen sowie $\mathrm{N}_{2} \mathrm{O}$-Konzentrationen $>1000 \mathrm{ppm}$ wurden nach gaschromatographischer Trennung (Carlo Erba, Fractovap 4200) mit einem Wärmeleitfähigkeitsdetektor (WLD) (HWD 450, Carlo Erba) ermittelt. Der Gaschromatograph ist mit zwei Trennsäulen ausgestattet. Die Trennung von $\mathrm{N}_{2}+\mathrm{O}_{2}, \mathrm{CO}_{2}$ und $\mathrm{N}_{2} \mathrm{O}$ findet in der Säule 1, die mit Porapak QS gefüllt ist, statt. Die zweite Säule enthält Molekularsieb $5 \AA$ und trennt $\mathrm{N}_{2}, \mathrm{O}_{2}$, und $\mathrm{CO}_{2} ; \mathrm{N}_{2} \mathrm{O}$ wird quantitativ absorbiert. Die Säulentemperatur beträgt $38^{\circ} \mathrm{C}$ und als Trägergas dient Helium (Reinheit 99,99 \%) zum Gastransport. Die Temperatur des Detektors beträgt $100^{\circ} \mathrm{C}$ und die des Filaments $130^{\circ} \mathrm{C}$. Die Bestimmung der Konzentration wird zum Teil an Einzelproben, zum Teil an 2 Parallelproben durchgeführt. Die Auswertung erfolgt über die Integrale der Peaks.

$\mathrm{N}_{2} \mathrm{O}$-Konzentrationen $<1000$ ppm wurden in einem Gaschromatopgraph mit einem Elektroneneinfang-Detektor (electron capture detector, ECD) gemessen. Der Gaschromatograph ist mit einer Vorsäule zur Trennung von $\mathrm{CO}_{2}$ und $\mathrm{H}_{2} \mathrm{O}$ versehen (Metallrohr, $250 \mathrm{~mm}$ × $6 \mathrm{~mm}$ innerer Durchmesser, je zur Hälfte gefüllt mit Ascarite und $\mathrm{Mg}\left(\mathrm{ClO}_{4}\right)_{2}$,). Die Trennsäule (Metallsäule $1500 \mathrm{~mm}$ x 2,5 mm) ist mit Porapak QS 5080 mesh gefüllt. Die isotherme Säulentemperatur beträgt $70^{\circ} \mathrm{C}$ und die Injektortemperatur $110^{\circ} \mathrm{C}$. Das Trägergas ist Argon-Methan (95/5) mit einer Flussrate von 18-20 ml/min. Der Detektor (ECD ${ }^{63} \mathrm{Ni}$ Carlo Erba, ECD 400) hat eine Referenzstromstärke von 1,7 nA, eine Pulsspannung von $15 \mathrm{~V}$, einen Pulsabstand von 0,1 $\mu$ s und eine Detektortemperatur von $350^{\circ} \mathrm{C}$. Durch die Verwendung des $\mathrm{CO}_{2}$-Absorbers können die Retentionszeiten und damit die Messdauer verkürzt werden, ohne dass der $\mathrm{N}_{2} \mathrm{O}$-Peak durch den $\mathrm{CO}_{2}$-Peak überlagert wird. Parallel zur Trennsäule liegt ein Bypass mit gleicher Flussrate des Trägergases. Die Gasströme der Trennsäule und des Bypasses können 
wahlweise mit Hilfe eines pneumatischen Ventils über den Detektor geleitet oder ins Freie entlassen werden. Auf diese Weise wird der $\mathrm{N}_{2}+\mathrm{O}_{2}$-Peak vom Detektor ferngehalten und dadurch eine Veränderung der Empfindlichkeit des ECD infolge Oxidation vermieden. Bei der Aufgabe der Gasprobe läuft reines Trägergas durch den Bypass über den Detektor. Das Mess-Trägergas wird über die Trennsäule ins Freie abgeführt, bis nach ca. 1,5 min. $\mathrm{N}_{2}$ und $\mathrm{O}_{2}$ entwichen sind. Dann wird der Gasstrom der Trennsäule zur $\mathrm{N}_{2} \mathrm{O}$-Messung über den Detektor geleitet, während das reine Trägergas des Bypasses ins Freie geleitet wird.

Die Injektion der Gasproben erfolgte über einen automatischen Probengeber (WELL, 2001).

\subsubsection{Massenspektrometrie}

Die Durchführung der massenspektrometrischen Untersuchungen erfolgte im Zentralen Isotopenlabor für Biologische und Medizinische Forschung der Universität Göttingen. Die Isotopenanalyse der Gase erfolgte durch Analyse der Massen 28 und 29 (WELL, et. al., 1998).

Die Messanlage besteht aus einem Elementaranalysator (NA 1500, Carlo-Erba) und einem Isotopen-Massenspektrometer (MAT 251, FINNIGAN).

Der Elementaranalysator ist mit dem Isotopenmassenspektrometer über eine TrappingBox (FINNIGAN, MAT 251) verbunden. Um den Gasstrom aus dem Elementaranalysator zu regeln und zu reinigen, wird ein Teil des Kapillarsystems sowie einige der Kühlfallen der Trapping-Box genutzt. Annähernd 1\% der Gasprobe wird direkt in die Ionenquelle des Massenspektrometers geleitet. Die Eliminierung des Sauerstoffs, sowie die Reduktion der Stickstoffoxide zu $\mathrm{N}_{2}$ findet durch den in den Elementaranalysator (Carlo Erba NA 1500) integrierten Reduktionsofen statt. Hierbei wird $\mathrm{N}_{2} \mathrm{O}$ in der Kupfersäule bei einer Temperatur von $650^{\circ} \mathrm{C} \mathrm{zu} \mathrm{N} \mathrm{N}_{2}$ reduziert. $\mathrm{CO}_{2}$ wird über eine gaschromatographische Säule, welche unmittelbar vor den Wärmeleitfähigkeitsdetektor installiert ist, abgetrennt.

Ein Standardgas $\left(\mathrm{N}_{2}\right.$, natürlicher ${ }^{15} \mathrm{~N}-$ Gehalt $)$ wird über den Trägergasstrom vor und nach jeder Analyse in das Massenspektrometer geleitet.

Die Intensitäten der Massen 28 und 29 werden simultan in einem MehrfachkollektorSystem bestimmt. 
Für eine vollständige Quantifizierung der $\left(\mathrm{N}_{2}+\mathrm{N}_{2} \mathrm{O}\right)$-Produktion mit der ${ }^{15} \mathrm{~N}$-Tracertechnik muss auch die Masse 30 berücksichtigt werden. Da in den Dauerinkubationsversuchen durch die Applikation von $\mathrm{K}^{15} \mathrm{NO}_{3}$ die ${ }^{15} \mathrm{~N}$-Anreicherung des Nitrats bekannt war, konnte die Masse 30 rechnerisch ermittelt werden. (WELL et al., 1999). Zur Kontrolle wurde an einigen Proben eine Parallelmessung mit der Technik der MikrowellenEquilibrierung (WELL et al., 1998) durchgeführt. Durch die Analyse von equilibrierten und nichtequilibrierten Parallelproben wird die Masse 30 indirekt bestimmt.

\section{Ergebnisse}

\subsection{Chemische Eigenschaften und Korngrößenzusammensetzung der Böden}

Tab. 5 und 6 zeigen die Ergebnisse der Schichtmittelwerte der bodenchemischen Parameter sowie der Korngrößenzusammensetzung jeweils für Lehm- und Sand-Böden. Bei dieser Auswertung wurden jeweils mehrere Messintervalle zu Schichten zusammengefasst, die im Hinblick auf den Reduktionsmittelvorrat $\left(\mathrm{C}_{\mathrm{org}}\right.$, Sulfid) relativ einheitlich waren. Diese Schichtgliederung wurde mit Hilfe der Tiefenverteilung der Gehalte an $\mathrm{C}_{\text {org. }}$ und Sulfid (Abb. 4) vorgenommen. Die Mittelwerte aus den Parallelbohrungen für jedes beprobte $20 \mathrm{~cm}$-Intervall finden sich im Anhang (Tab. A1, A2 und A3). Bei den

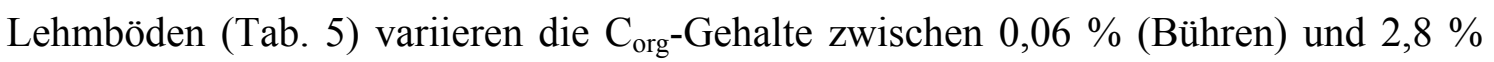
(Schladen). Bei den Auen-Gleyen (Reinshof, Aher Kämpe, Schladen) nimmt der $\mathrm{C}_{\text {org}^{-}}$ Gehalt im Bereich der Grundwasseroberfläche nach unten hin sprunghaft zu.

Der Schwankungsbereich des heißwasserlöslichen Kohlenstoffs $\left(\mathrm{C}_{\mathrm{hwl}}\right)$ erstreckt sich von $72,3 \mathrm{mg} \mathrm{kg}^{-1}$ (Bühren) bis $843 \mathrm{mg} \mathrm{kg}^{-1}$ (Aher Kämpe).

Die Konzentrationen an löslichem Kohlenstoff $\left(\mathrm{C}_{\text {lös }}\right)$ bewegen sich zwischen 7,6 mg $\mathrm{kg}^{-1}$ und $38,8 \mathrm{mg} \mathrm{kg}^{-1}$ wobei Bühren wiederum die geringsten Werte aufweist.

Die Sulfid-Gehalte variieren sehr stark. Dies ist sowohl innerhalb eines Standortes als auch zwischen den beprobten Flächen zu erkennen. Die Sulfid-Gehalte nehmen bei allen sulfidhaltigen Profilen unterhalb des Grundwasserspiegels mit zunehmender Tiefe kontinuierlich zu (s. Abb. 4). Dies ist in Neuhäuser Felde besonders stark ausgeprägt. Der höchste Wert liegt bei $7905 \mathrm{mg} \mathrm{kg}^{-1}$ (Reinshof). Der Auen-Pseudogley (Bühren) ist der einzige fast sulfidfreie Lehm-Boden. 
Tab. 5: Schichtmittelwerte der bodenchemischen Parameter sowie der Korngrößenzusammensetzung der untersuchten Lehm-Böden

\begin{tabular}{|c|c|c|c|c|c|c|c|c|c|c|c|c|c|}
\hline \multirow[t]{2}{*}{ Standort } & \multirow{2}{*}{$\begin{array}{l}\text { Schicht- } \\
\text { folge } \\
\text { (cm) }\end{array}$} & \multirow{2}{*}{$\begin{array}{c}\mathrm{C}_{\text {org }} \\
\%\end{array}$} & \multirow[t]{2}{*}{$\mathrm{C}_{\mathrm{hwl}}$} & \multirow{2}{*}{$\begin{array}{l}\text { DOC } \\
\mathrm{mg} / \mathrm{kg}\end{array}$} & \multirow[t]{2}{*}{ Sulfid } & \multirow[t]{2}{*}{$\mathrm{Fe}_{\text {lös }}$} & \multirow[t]{2}{*}{$\mathrm{C} / \mathrm{N}$} & \multirow[t]{2}{*}{$\begin{array}{l}\mathrm{pH} \\
\left(\mathrm{CaCl}_{2}\right)\end{array}$} & \multirow[t]{2}{*}{$\mathrm{CaCO}_{3}$} & \multirow[t]{2}{*}{ Sand } & \multirow{2}{*}{$\begin{array}{l}\text { Schluff } \\
\%\end{array}$} & \multirow[t]{2}{*}{ Ton } & \multirow[t]{2}{*}{$\mathbf{N}$} \\
\hline & & & & & & & & & & & & & \\
\hline $\begin{array}{l}\text { Aher- } \\
\text { Kämpe }\end{array}$ & $140-210$ & $\begin{array}{l}2,02 \\
\pm 0,73\end{array}$ & n.b. & $\begin{array}{l}38,8 \\
\pm 18,3\end{array}$ & $\begin{array}{l}919 \\
\pm 1205\end{array}$ & $\begin{array}{l}0,1 \\
\pm 0,1\end{array}$ & $\begin{array}{l}10,6 \\
\pm 0,8\end{array}$ & $\begin{array}{l}6,7 \\
\pm 0,4\end{array}$ & $\begin{array}{l}3,7 \\
\pm 2,6\end{array}$ & $\begin{array}{l}0,7 \\
\pm 0,4\end{array}$ & $\begin{array}{l}61 \\
\pm 6\end{array}$ & $\begin{array}{l}39 \\
\pm 6\end{array}$ & 10 \\
\hline $\begin{array}{l}\text { Aher- } \\
\text { Kämpe }\end{array}$ & $210-240$ & $\begin{array}{l}0,78 \\
\pm 0,60\end{array}$ & $\begin{array}{l}843 \\
\pm 293\end{array}$ & $\begin{array}{l}30,7 \\
\pm 14,7\end{array}$ & $\begin{array}{l}614 \\
\pm 546\end{array}$ & $\begin{array}{l}0,1 \\
\pm 0\end{array}$ & $\begin{array}{l}12,5 \\
\pm 0,3\end{array}$ & $\begin{array}{l}7,2 \\
\pm 0,4\end{array}$ & $\begin{array}{l}1,9 \\
\pm 0,8\end{array}$ & $\begin{array}{l}5,8 \\
\pm 0\end{array}$ & $\begin{array}{l}67 \\
\pm 0\end{array}$ & $\begin{array}{l}27 \\
\pm 0\end{array}$ & 3 \\
\hline Bühren & $200-260$ & $\begin{array}{l}0,20 \\
\pm 0,08\end{array}$ & $\begin{array}{l}138 \\
\pm 147\end{array}$ & $\begin{array}{l}7,6 \\
\pm 1,6\end{array}$ & n.n. & $\begin{array}{l}0,3 \\
\pm 0,1\end{array}$ & $\begin{array}{l}7,5 \\
\pm 0,5\end{array}$ & $\begin{array}{l}5,5 \\
\pm 0,1\end{array}$ & 0,00 & $\begin{array}{l}4,6 \\
\pm 2,5\end{array}$ & $\begin{array}{l}61 \\
\pm 2,8\end{array}$ & $\begin{array}{l}35 \\
\pm 3\end{array}$ & 9 \\
\hline Bühren & $260-320$ & $\begin{array}{l}0,06 \\
\pm 0,05\end{array}$ & $\begin{array}{l}72,3 \\
\pm 49,8\end{array}$ & $\begin{array}{l}8,5 \\
\pm 1,9\end{array}$ & $\begin{array}{l}9,5 \\
\pm 6,6\end{array}$ & $\begin{array}{l}0,2 \\
\pm 0,1\end{array}$ & $\begin{array}{l}8,6 \\
\pm 6,2\end{array}$ & $\begin{array}{l}5,9 \\
\pm 0,3\end{array}$ & 0,00 & $\begin{array}{l}6,2 \\
\pm 6,4\end{array}$ & $\begin{array}{l}64 \\
\pm 6,4\end{array}$ & $\begin{array}{l}29 \\
\pm 7\end{array}$ & 9 \\
\hline Reinshof & $100-130$ & $\begin{array}{l}0,56 \\
\pm 0,23\end{array}$ & $\begin{array}{l}324 \\
\pm 281\end{array}$ & n.b. & $\begin{array}{l}67,1 \\
\pm 16,5\end{array}$ & n.b. & $\begin{array}{l}8,6 \\
\pm 0,9\end{array}$ & $\begin{array}{l}7,03 \\
\pm 0,24\end{array}$ & $\begin{array}{l}0,7 \\
\pm 0,2\end{array}$ & $\begin{array}{l}8,4 \\
\pm 2,3\end{array}$ & $\begin{array}{l}50 \\
\pm 5,2\end{array}$ & $\begin{array}{l}42 \\
\pm 5\end{array}$ & 6 \\
\hline Reinshof & $130-140$ & $\begin{array}{l}0,48 \\
(0,0)\end{array}$ & n.b. & n.b. & $\begin{array}{l}112 \\
(22)\end{array}$ & n.b. & $\begin{array}{l}9,2 \\
(0,1)\end{array}$ & $\begin{array}{l}6,9 \\
(0,0)\end{array}$ & $\begin{array}{l}0,4 \\
(0,2)\end{array}$ & $\begin{array}{l}5,2 \\
(0,0)\end{array}$ & $\begin{array}{l}68 \\
(0)\end{array}$ & $\begin{array}{l}27 \\
(0)\end{array}$ & 2 \\
\hline Reinshof & $140-180$ & $\begin{array}{l}2,29 \\
\pm 1,65\end{array}$ & $\begin{array}{l}507 \\
\pm 264\end{array}$ & n.b. & $\begin{array}{l}1391 \\
\pm 875\end{array}$ & n.b. & $\begin{array}{l}16,4 \\
\pm 4,1\end{array}$ & $\begin{array}{l}6,8 \\
\pm 0,4\end{array}$ & $\begin{array}{l}5,3 \\
\pm 5,5\end{array}$ & $\begin{array}{l}26,7 \\
\pm 13\end{array}$ & $\begin{array}{l}58,3 \\
\pm 7,3\end{array}$ & $\begin{array}{l}15 \\
\pm 5,7\end{array}$ & 8 \\
\hline Reinshof & $180-200$ & $\begin{array}{l}13,61 \\
\pm 13,7\end{array}$ & $\begin{array}{l}656 \\
\pm 35\end{array}$ & n.b. & $\begin{array}{l}7905 \\
\pm 8329\end{array}$ & n.b. & $\begin{array}{l}18,4 \\
\pm 6,9\end{array}$ & $\begin{array}{l}6,5 \\
\pm 1\end{array}$ & $\begin{array}{l}2,5 \\
\pm 4,4\end{array}$ & $\begin{array}{l}32,7 \\
\pm 0\end{array}$ & $\begin{array}{l}55 \\
\pm 0\end{array}$ & $\begin{array}{l}12,3 \\
\pm 0\end{array}$ & 4 \\
\hline Schladen & $160-180$ & $\begin{array}{l}0,45 \\
\pm 0,04\end{array}$ & n.b. & $\begin{array}{l}19 \\
\pm 3,3\end{array}$ & $\begin{array}{l}15,3 \\
\pm 9,0\end{array}$ & $\begin{array}{l}0,1 \\
\pm 0,0\end{array}$ & $\begin{array}{l}9,1 \\
\pm 0,7\end{array}$ & $\begin{array}{l}6,9 \\
\pm 0,2\end{array}$ & $\begin{array}{l}0,4 \\
\pm 0,4\end{array}$ & $\begin{array}{l}6,7 \\
\pm 0,0\end{array}$ & $\begin{array}{l}77 \\
\pm 0,0\end{array}$ & $\begin{array}{l}16,5 \\
\pm 0,0\end{array}$ & 3 \\
\hline Schladen & $180-240$ & $\begin{array}{l}2,0 \\
\pm 1,33\end{array}$ & n.b. & $\begin{array}{l}23,6 \\
\pm 6,2\end{array}$ & $\begin{array}{l}85 \\
\pm 135\end{array}$ & $\begin{array}{l}0,1 \\
\pm 0,1\end{array}$ & $\begin{array}{l}13,7 \\
\pm 3,6\end{array}$ & $\begin{array}{l}5,9 \\
\pm 0,5\end{array}$ & $\begin{array}{l}0,1 \\
\pm 0,2\end{array}$ & $\begin{array}{l}20,1 \\
\pm 6,0\end{array}$ & $\begin{array}{l}65,2 \\
\pm 8,5\end{array}$ & $\begin{array}{l}14,8 \\
\pm 2,7\end{array}$ & 9 \\
\hline Schladen & $240-340$ & $\begin{array}{l}2,78 \\
\pm 1,38\end{array}$ & n.b. & $\begin{array}{l}19,1 \\
\pm 5,5\end{array}$ & $\begin{array}{l}664 \\
\pm 69\end{array}$ & $\begin{array}{l}0,4 \\
\pm 0,6\end{array}$ & $\begin{array}{l}20 \\
\pm 2\end{array}$ & $\begin{array}{l}4,6 \\
\pm 1,5\end{array}$ & $\begin{array}{l}0,0 \\
\pm 0,0\end{array}$ & $\begin{array}{l}51,5 \\
\pm 2,8\end{array}$ & $\begin{array}{l}29,1 \\
\pm 7,2\end{array}$ & $\begin{array}{l}19,4 \\
\pm 4,4\end{array}$ & 12 \\
\hline $\begin{array}{l}\text { Neuhäuser- } \\
\text { Felde }\end{array}$ & $100-240$ & $\begin{array}{l}0,30 \\
\pm 0,22\end{array}$ & $\begin{array}{l}305 \\
\pm 120\end{array}$ & $\begin{array}{l}23,8 \\
\pm 7,4\end{array}$ & n.n. & $\begin{array}{l}0,1 \\
\pm 0,1\end{array}$ & $\begin{array}{l}9,5 \\
\pm 4,4\end{array}$ & $\begin{array}{l}6,4 \\
\pm 0,4\end{array}$ & $\begin{array}{l}0,9 \\
\pm 1,7\end{array}$ & $\begin{array}{l}29 \\
\pm 13\end{array}$ & $\begin{array}{l}54,1 \\
\pm 7,6\end{array}$ & $\begin{array}{l}17,4 \\
\pm 9,3\end{array}$ & 21 \\
\hline $\begin{array}{l}\text { Neuhäuser- } \\
\text { Felde }\end{array}$ & $240-360$ & $\begin{array}{l}0,57 \\
\pm 0,22\end{array}$ & $\begin{array}{l}747 \\
\pm 299\end{array}$ & $\begin{array}{l}32 \\
\pm 11\end{array}$ & $\begin{array}{l}973 \\
\pm 778\end{array}$ & $\begin{array}{l}0,1 \\
\pm 0,1\end{array}$ & $\begin{array}{l}11,5 \\
\pm 2,1\end{array}$ & $\begin{array}{l}6,8 \\
\pm 0,2\end{array}$ & $\begin{array}{l}6,4 \\
\pm 0,8\end{array}$ & $\begin{array}{l}29 \\
\pm 13\end{array}$ & $\begin{array}{l}57,4 \\
\pm 9,9\end{array}$ & $\begin{array}{l}13,8 \\
\pm 4,7\end{array}$ & 14 \\
\hline $\begin{array}{l}\text { Neuhäuser- } \\
\text { Felde }\end{array}$ & $360-400$ & $\begin{array}{l}0,44 \\
(0,09)\end{array}$ & $\begin{array}{l}575 \\
(96)\end{array}$ & $\begin{array}{l}21 \\
(30)\end{array}$ & $\begin{array}{l}941 \\
(60)\end{array}$ & $\begin{array}{l}0 \\
(0)\end{array}$ & $\begin{array}{l}13,6 \\
(2,8)\end{array}$ & $\begin{array}{l}6,72 \\
(0,01)\end{array}$ & $\begin{array}{l}7,7 \\
(0,2)\end{array}$ & $\begin{array}{l}52,7 \\
(6,6)\end{array}$ & $\begin{array}{l}41,6 \\
(5,1)\end{array}$ & $\begin{array}{l}5,7 \\
(1,5)\end{array}$ & 2 \\
\hline
\end{tabular}

( ) $\quad=$ Differenz

$\pm \quad=$ Standardabweichung

$\mathrm{N} \quad=$ Anzahl der Proben

n. n. = nicht nachweisbar

n. b. = nicht bestimmt 
Tab. 6: Schichtmittelwerte der bodenchemischen Parameter sowie der Korngrößenzusammensetzung der untersuchten Sand-Böden

\begin{tabular}{|c|c|c|c|c|c|c|c|c|c|c|c|c|c|}
\hline \multirow[t]{2}{*}{ Standort } & \multirow{2}{*}{$\begin{array}{l}\text { Schicht- } \\
\text { folge } \\
\text { (cm) }\end{array}$} & \multirow{2}{*}{$\begin{array}{c}\mathrm{C}_{\text {org }} \\
\%\end{array}$} & \multirow[t]{2}{*}{$C_{\text {hwl }}$} & \multirow{2}{*}{$\begin{array}{l}\text { DOC } \\
\mathrm{mg} / \mathrm{kg}\end{array}$} & \multirow[t]{2}{*}{ Sulfid } & \multirow[t]{2}{*}{$\mathrm{Fe}_{\text {lös }}$} & \multirow[t]{2}{*}{$C / N$} & \multirow{2}{*}{$\begin{array}{l}\mathrm{pH} \\
\left(\mathrm{CaCl}_{2}\right)\end{array}$} & \multirow[t]{2}{*}{$\mathrm{CaCO}_{3}$} & \multirow[t]{2}{*}{ Sand } & \multirow{2}{*}{$\begin{array}{l}\text { Schluff } \\
\%\end{array}$} & \multirow[t]{2}{*}{ Ton } & \multirow[t]{2}{*}{$\mathbf{N}$} \\
\hline & & & & & & & & & & & & & \\
\hline Dinklage & $120-160$ & $\begin{array}{l}0,14 \\
\pm 0,04\end{array}$ & $\begin{array}{l}174 \\
\pm 40\end{array}$ & $\begin{array}{l}16,3 \\
\pm 10,7\end{array}$ & $\begin{array}{l}2,4 \\
\pm 1,3\end{array}$ & $\begin{array}{l}0,9 \\
\pm 0,4\end{array}$ & $\begin{array}{l}25,8 \\
\pm 9,6\end{array}$ & $\begin{array}{l}4,3 \\
\pm 0,2\end{array}$ & $\begin{array}{l}0,0 \\
\pm 0,0\end{array}$ & $\begin{array}{l}66 \\
\pm 36\end{array}$ & $\begin{array}{l}15,4 \\
\pm 15,4\end{array}$ & $\begin{array}{l}1,9 \\
\pm 2,1\end{array}$ & 6 \\
\hline Dinklage & $160-240$ & $\begin{array}{l}0,12 \\
\pm 0,04\end{array}$ & $\begin{array}{l}155 \\
\pm 36\end{array}$ & $\begin{array}{l}9,9 \\
\pm 4,5\end{array}$ & $\begin{array}{l}5,5 \\
\pm 4,4\end{array}$ & $\begin{array}{l}1,1 \\
\pm 1,9\end{array}$ & $\begin{array}{l}22,6 \\
\pm 5,5\end{array}$ & $\begin{array}{l}4,8 \\
\pm 0,7\end{array}$ & $\begin{array}{l}0,0 \\
\pm 0,0\end{array}$ & $\begin{array}{l}78 \\
\pm 9,8\end{array}$ & $\begin{array}{l}20,2 \\
\pm 9\end{array}$ & $\begin{array}{l}1,8 \\
\pm 0,9\end{array}$ & 15 \\
\hline Dinklage & $240-400$ & $\begin{array}{l}0,17 \\
\pm 0,05\end{array}$ & $\begin{array}{l}189 \\
\pm 36\end{array}$ & $\begin{array}{l}7,2 \\
\pm 2,7\end{array}$ & $\begin{array}{l}20,6 \\
\pm 12,9\end{array}$ & $\begin{array}{l}38,6 \\
\pm 38,5\end{array}$ & $\begin{array}{l}22,4 \\
\pm 9,5\end{array}$ & $\begin{array}{l}4,6 \\
\pm 0,2\end{array}$ & $\begin{array}{l}0,0 \\
\pm 0,0\end{array}$ & $\begin{array}{l}73,9 \\
\pm 5,5\end{array}$ & $\begin{array}{l}24,4 \\
\pm 5,5\end{array}$ & $\begin{array}{l}1,6 \\
\pm 0,4\end{array}$ & 10 \\
\hline $\begin{array}{c}\text { Freistatt * } \\
1\end{array}$ & $140-300$ & $\begin{array}{l}0,18 \\
\pm 0,03\end{array}$ & $\begin{array}{l}484 \\
\pm 51\end{array}$ & $\begin{array}{l}33 \\
\pm 7\end{array}$ & $\begin{array}{l}3,9 \\
\pm 3,7\end{array}$ & $\begin{array}{l}1 \\
\pm 1\end{array}$ & $\begin{array}{l}42 \\
\pm 6\end{array}$ & $\begin{array}{l}4,4 \\
\pm 0,1\end{array}$ & $\begin{array}{l}0,0 \\
\pm 0,0\end{array}$ & n. b. & n. b. & n. b. & 7 \\
\hline $\begin{array}{c}\text { Freistatt * } \\
2\end{array}$ & $160-300$ & $\begin{array}{l}0,13 \\
\pm 0,09\end{array}$ & $\begin{array}{l}327 \\
\pm 132\end{array}$ & $\begin{array}{l}36 \\
\pm 15\end{array}$ & $\begin{array}{l}4,4 \\
\pm 1,6\end{array}$ & 0,00 & $\begin{array}{l}30 \\
\pm 10\end{array}$ & $\begin{array}{l}4,4 \\
\pm 0,1\end{array}$ & $\begin{array}{l}0,0 \\
\pm 0,0\end{array}$ & n. b. & n. b. & n. b. & 7 \\
\hline $\begin{array}{c}\text { Freistatt * } \\
3\end{array}$ & $160-300$ & $\begin{array}{l}0,06 \\
\pm 0,01\end{array}$ & $\begin{array}{l}109 \\
\pm 19\end{array}$ & $\begin{array}{l}7,8 \\
\pm 1,1\end{array}$ & $\begin{array}{l}6,1 \\
\pm 2,8\end{array}$ & 0,00 & $\begin{array}{l}9,5 \\
\pm 1,8\end{array}$ & $\begin{array}{l}4,2 \\
\pm 0,1\end{array}$ & $\begin{array}{l}0,0 \\
\pm 0,0\end{array}$ & n. b. & n. b. & n. b. & 7 \\
\hline Fuhrberg & $\begin{array}{l}100-160 \\
360-400\end{array}$ & $\begin{array}{l}0,12 \\
\pm 0,13\end{array}$ & $\begin{array}{l}377 \\
\pm 235\end{array}$ & $\begin{array}{l}9,6 \\
\pm 0,2\end{array}$ & $\begin{array}{l}1,5 \\
\pm 2,2\end{array}$ & $\begin{array}{l}0,2 \\
\pm 0\end{array}$ & $\begin{array}{l}18,9 \\
\pm 7,6\end{array}$ & $\begin{array}{l}5,2 \\
\pm 0,2\end{array}$ & $\begin{array}{l}0,0 \\
\pm 0,0\end{array}$ & $\begin{array}{l}96 \\
\pm 0\end{array}$ & $\begin{array}{l}0,2 \\
\pm 0\end{array}$ & $\begin{array}{l}4,3 \\
\pm 0\end{array}$ & 5 \\
\hline Fuhrberg & $160-360$ & $\begin{array}{l}0,09 \\
\pm 0,03\end{array}$ & $\begin{array}{l}319 \\
\pm 67\end{array}$ & $\begin{array}{l}11,7 \\
\pm 3,6\end{array}$ & $\begin{array}{l}0,5 \\
\pm 0,7\end{array}$ & $\begin{array}{l}0,3 \\
\pm 0,1\end{array}$ & $\begin{array}{l}21,6 \\
\pm 6,6\end{array}$ & $\begin{array}{l}5 \\
\pm 0,2\end{array}$ & $\begin{array}{l}0,0 \\
\pm 0,0\end{array}$ & $\begin{array}{l}98,5 \\
\pm 0,7\end{array}$ & $\begin{array}{l}0,1 \\
\pm 0\end{array}$ & $\begin{array}{l}1,3 \\
\pm 0,7\end{array}$ & 10 \\
\hline $\begin{array}{l}\text { Mark- } \\
\text { hausen }\end{array}$ & $150-230$ & $\begin{array}{l}0,79 \\
\pm 0,97\end{array}$ & $\begin{array}{l}253 \\
\pm 99\end{array}$ & $\begin{array}{l}25,9 \\
\pm 11,1\end{array}$ & $\begin{array}{l}80 \\
\pm 117\end{array}$ & $\begin{array}{l}1,6 \\
\pm 1,7\end{array}$ & $\begin{array}{l}51 \\
\pm 23\end{array}$ & $\begin{array}{l}4,5 \\
\pm 0,2\end{array}$ & $\begin{array}{l}0,0 \\
\pm 0,0\end{array}$ & $\begin{array}{l}95 \\
\pm 0\end{array}$ & $\begin{array}{l}2 \\
\pm 0\end{array}$ & $\begin{array}{l}2,7 \\
\pm 0\end{array}$ & 12 \\
\hline $\begin{array}{l}\text { Mark- } \\
\text { hausen }\end{array}$ & $230-310$ & $\begin{array}{l}0,21 \\
\pm 0,21\end{array}$ & $\begin{array}{l}140 \\
\pm 53\end{array}$ & $\begin{array}{l}20,6 \\
\pm 6,3\end{array}$ & $\begin{array}{l}47 \\
\pm 53\end{array}$ & $\begin{array}{l}7,19 \\
\pm 9,96\end{array}$ & $\begin{array}{l}39 \\
\pm 23\end{array}$ & $\begin{array}{l}4,7 \\
\pm 0,2\end{array}$ & $\begin{array}{l}0,0 \\
\pm 0,0\end{array}$ & $\begin{array}{l}95 \\
\pm 0\end{array}$ & $\begin{array}{l}2 \\
\pm 0\end{array}$ & $\begin{array}{l}2,7 \\
\pm 0\end{array}$ & 11 \\
\hline Oldendorf & $100-120$ & $\begin{array}{l}0,16 \\
\pm 0,07\end{array}$ & $\begin{array}{l}202 \\
\pm 73\end{array}$ & $\begin{array}{l}14,7 \\
\pm 3,6\end{array}$ & $\begin{array}{l}64 \\
\pm 52\end{array}$ & $\begin{array}{l}1,1 \\
\pm 1,1\end{array}$ & $\begin{array}{l}39 \\
\pm 18\end{array}$ & $\begin{array}{l}5,5 \\
\pm 0,3\end{array}$ & $\begin{array}{l}0,0 \\
\pm 0,0\end{array}$ & $\begin{array}{l}93 \\
\pm 0\end{array}$ & $\begin{array}{l}5,9 \\
\pm 0\end{array}$ & $\begin{array}{l}1 \\
\pm 0\end{array}$ & 7 \\
\hline Oldendorf & $120-140$ & $\begin{array}{l}0,19 \\
\pm 0,11\end{array}$ & $\begin{array}{l}219 \\
\pm 77\end{array}$ & $\begin{array}{l}11,2 \\
\pm 5,3\end{array}$ & $\begin{array}{l}105 \\
\pm 67\end{array}$ & $\begin{array}{l}1,3 \\
\pm 1,6\end{array}$ & $\begin{array}{l}28,2 \\
\pm 7,5\end{array}$ & $\begin{array}{l}5,4 \\
\pm 0,4\end{array}$ & $\begin{array}{l}0 \\
\pm 0,1\end{array}$ & $\begin{array}{l}93 \\
\pm 0\end{array}$ & $\begin{array}{l}5,9 \\
\pm 0\end{array}$ & $\begin{array}{l}1 \\
\pm 0\end{array}$ & 7 \\
\hline Oldendorf & $140-160$ & $\begin{array}{l}0,18 \\
\pm 0,11\end{array}$ & $\begin{array}{l}191 \\
\pm 55\end{array}$ & $\begin{array}{l}11,4 \\
\pm 6,8\end{array}$ & $\begin{array}{l}120 \\
\pm 85\end{array}$ & $\begin{array}{l}1,8 \\
\pm 2,6\end{array}$ & $\begin{array}{l}31 \\
\pm 13\end{array}$ & $\begin{array}{l}5,5 \\
\pm 0,3\end{array}$ & $\begin{array}{l}0,1 \\
\pm 0,2\end{array}$ & $\begin{array}{l}93 \\
\pm 0\end{array}$ & $\begin{array}{l}5,9 \\
\pm 0\end{array}$ & $\begin{array}{l}1 \\
\pm 0\end{array}$ & 6 \\
\hline Vechtel & $80-120$ & $\begin{array}{l}0,10 \\
\pm 0,02\end{array}$ & $\begin{array}{l}150 \\
\pm 25\end{array}$ & $\begin{array}{l}23,9 \\
\pm 7,6\end{array}$ & $\begin{array}{l}3,4 \\
\pm 1,4\end{array}$ & $\begin{array}{l}0,2 \\
\pm 0,1\end{array}$ & $\begin{array}{l}29 \\
\pm 13\end{array}$ & $\begin{array}{l}5,2 \\
\pm 0,1\end{array}$ & $\begin{array}{l}0,0 \\
\pm 0,0\end{array}$ & $\begin{array}{l}93 \\
\pm 0\end{array}$ & $\begin{array}{l}5,9 \\
\pm 0\end{array}$ & $\begin{array}{l}1 \\
\pm 0\end{array}$ & 9 \\
\hline Vechtel & $120-140$ & $\begin{array}{l}0,09 \\
\pm 0,02\end{array}$ & $\begin{array}{l}152 \\
\pm 25\end{array}$ & $\begin{array}{l}19 \\
\pm 2,9\end{array}$ & $\begin{array}{l}4,1 \\
\pm 0,9\end{array}$ & $\begin{array}{l}0,3 \\
\pm 0,1\end{array}$ & $\begin{array}{l}33,5 \\
\pm 13,7\end{array}$ & $\begin{array}{l}5,2 \\
\pm 0,2\end{array}$ & $\begin{array}{l}0,0 \\
\pm 0,0\end{array}$ & $\begin{array}{l}93 \\
\pm 0\end{array}$ & $\begin{array}{l}5,9 \\
\pm 0\end{array}$ & $\begin{array}{l}1 \\
\pm 0\end{array}$ & 5 \\
\hline $\begin{array}{l}=\mathrm{A} \\
=\mathrm{L}\end{array}$ & inl der & en & & & & & & & & & & & \\
\hline
\end{tabular}


Die Gehalte an löslichem Eisen ( $\mathrm{Fe}_{\text {lös }}$ ) liegen zwischen 0,02 und $0,39 \mathrm{mg} \mathrm{kg}^{-1}$ und lassen kein flächenspezifisches Muster erkennen.

Die C/N-Verhältnisse, die ein Maß für die Qualität organischer Substanz des Bodens sind, liegen in einem engem Bereich, nämlich zwischen 7,5 und 19,8 (Tab. 5). Innerhalb des Tiefen-Profils von Bühren (Pseudo-Gley) variieren die Verhältnisse nur geringfügig, während bei den Auen-Gleyen innerhalb der verschiedenen Tiefen eines Profils die Schwankungen der Verhältnisse größer sind. Im Profil Reinshof ist mit der Tiefe sogar eine Verdoppelung zu beobachten.

Die pH-Werte liegen hier im schwach sauren Bereich, während sie bei den übrigen Böden überwiegend im neutralen Bereich liegen.

Bei den durchgeführten Untersuchungen wurden unterschiedlichen Kalkgehalte $(0,0$ 7,7 \%) ermittelt. Bühren ist das einzige Beispiel mit vollständig kalkfreien Bodenprofil. In den sechs untersuchten Sand-Böden (Tab. 6) (Gley-Podsol, Podsol-Gley) sind die

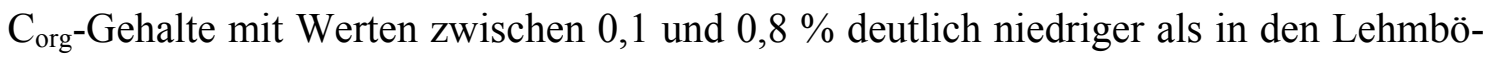
den. Abb. 4 zeigt die Tiefendynamik der Reduktionsmittelvorräte der Sandböden. Hierbei sind die Gehalte über die Tiefe recht konstant mit Ausnahme vom Standort Markhausen (Tiefumbruchboden), wo ein ausgeprägtes Maximum unter der Grundwasseroberfläche vorhanden ist.

Der heißwasserlösliche Kohlenstoff $\left(\mathrm{C}_{\mathrm{hwl}}\right)$ schwankt zwischen 150 und $484 \mathrm{mg} \mathrm{kg}{ }^{-1}$. Auch hier sind keine auffälligenn Unterschiede zwischen den Standorten erkennbar.

Die Gehalte an $C_{\text {lös }}$ liegen zwischen 7,2 $\mathrm{mg} \mathrm{kg}^{-1}$ und 35,9 $\mathrm{mg} \mathrm{kg}^{-1}$ (Tab. 6). Innerhalb der Profile bestehen keine große Unterschiede.

Die Sulfid-Gehalte sind bei allen Standorten gering oder nicht nachweisbar (Abb. 4, Tab. 6). Die höchsten Werte treten an den Standorten Oldendorf und Markhausen unterhalb der Grundwasseroberfläche auf, wo auch die $\mathrm{C}_{\text {org }}$-Gehalte am höchsten sind.

Mit Ausnahme von Dinklage (38,6 $\left.\mathrm{mg} \mathrm{kg}^{-1}\right)$ liegen die Gehalte an löslichen Eisen ( $\left.\mathrm{Fe}_{\text {lös }}\right)$ bei allen Proben auf geringem Niveau zwischen 0,12 und 7,19 $\mathrm{mg} \mathrm{kg}^{-1}$.

Die C/N-Verhältnisse sind im Vergleich zu den Lehmböden mit Werten zwischen 9 und 51 sehr viel weiter. Bei keinem der Sandböden wurde $\mathrm{CaCO}_{3}$ nachgewiesen.

Die pH-Werte liegen im schwach sauren bis sauren Bereich (4,3 - 5,5).

Bei der Körnung fällt der Standort Dinklage durch vergleichsweise hohe Schluffgehalte auf. Die übrigen Böden sind mit Sandgehalten über $95 \%$ als reine Sande einzustufen. Die Tongehalte liegen zwischen 1 und 5\%. 

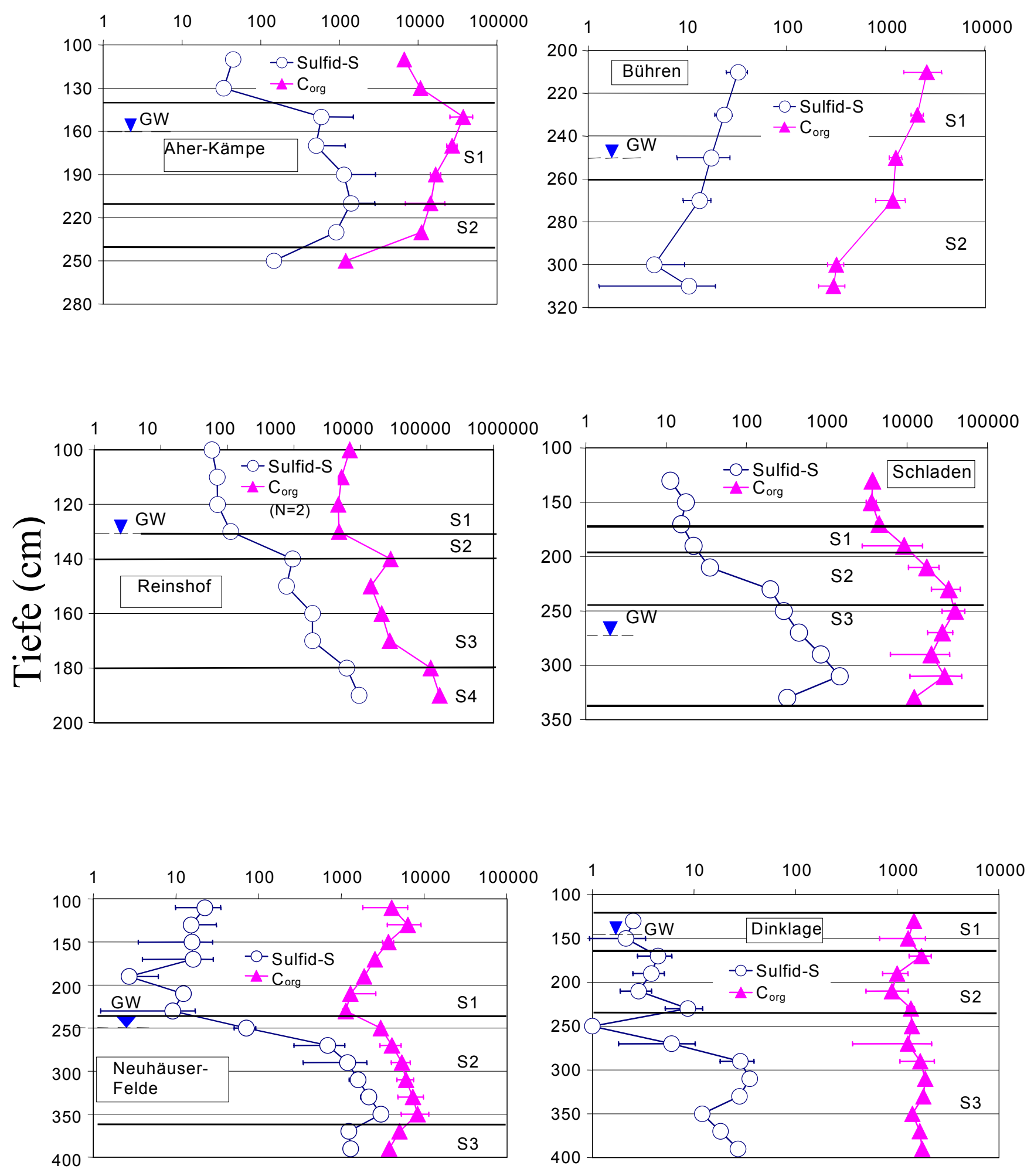

\section{Gehalt an Sulfid und $\mathrm{C}_{\text {org }}\left(\mathrm{mg} \mathrm{kg}^{-1}\right)$}

\footnotetext{
Abb. 4: Tiefenverteilung der Reduktionsmittelvorräte an den untersuchten Standorten

$\mathrm{GW} \quad=$ Grundwasserstand

$\mathrm{S} \quad=$ Schicht
} 

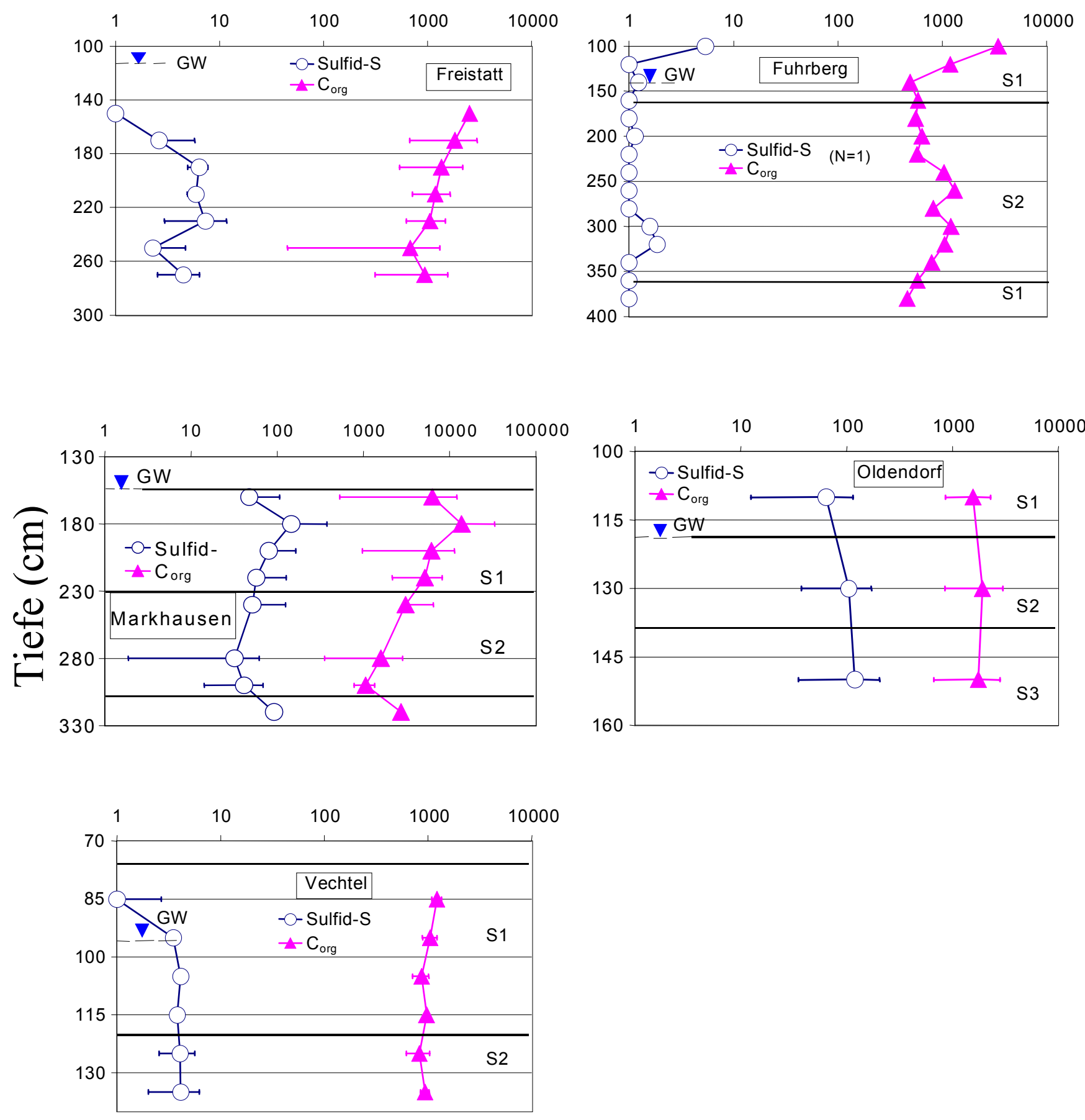

Gehalt an Sulfid und $\mathrm{C}_{\text {org }}\left(\mathrm{mg} \mathrm{kg}^{-1}\right)$

\section{Abb. 4 (Fortsetzung): Tiefenverteilung der Reduktionsmittelvorräte an den untersuchten Standorten}

$\begin{array}{ll}\mathrm{GW} & =\text { Grundwasserstand } \\ \mathrm{S} & =\text { Schicht }\end{array}$ 


\subsection{Messergebnisse der anaeroben Inkubationsversuche}

Die Zeitverläufe der Inkubationsversuche zur Reduktion von Nitrat sowie zur Produktion von Sulfat-, $\mathrm{CO}_{2}$ - und ${ }^{15}\left(\mathrm{~N}_{2}+\mathrm{N}_{2} \mathrm{O}\right)$ bei den untersuchten Böden sind der Abb. $5 \mathrm{zu}$ entnehmen. Die Nachweisgrenzen für die Umsatzraten waren abhängig vom Inkubationsintervall, von der analytischen Präzision sowie von zusätzlichen methodischen Unsicherheiten. Die Präzisionen für ${ }^{15}\left(\mathrm{~N}_{2}+\mathrm{N}_{2} \mathrm{O}\right), \mathrm{SO}_{4}{ }^{-} \mathrm{S}$ und $\mathrm{NO}_{3}{ }^{-}-\mathrm{N}$ betrugen 6,1 ppm, 0,1 $\mathrm{mg} \mathrm{l}^{-1}$ und 0,2 $\mathrm{mg} \mathrm{l}^{-1}$. Die Nachweisgrenze für die ${ }^{15}\left(\mathrm{~N}_{2}+\mathrm{N}_{2} \mathrm{O}\right)$-Produktion betrug zwischen $0,014 \mathrm{mg} \mathrm{N} \mathrm{kg}^{-1} \mathrm{~d}^{-1}$ (24 h Inkubationszeit) und $0,35 \mathrm{mg} \mathrm{N} \mathrm{kg}^{-1} \mathrm{~d}^{-1}$ (1 h Inkubationszeit). Für die Abschätzung der Nachweisgrenze der Sulfatproduktion war die Variation der Hintergrundkonzentration an Sulfat (vgl. Kap. 4.4.3.1) zu berücksichtigen. Diese betrug in den untersuchten Böden zwischen 5,3 und $38,8 \mathrm{mg} \mathrm{S} \mathrm{kg}^{-1}$ und war somit immer wesentlich höher als die analytische Präzision. Die Analyse der Sulfathintergrundwerte wurde jeweils an Parallelproben der Inkubationsversuche durchgeführt. Zur Bestimmung der anaeroben Sulfatproduktion wurden somit die Hintergrundwerte der Parallelproben von den Sulfatkonzentrationen der Proben aus den anaeroben Inkubation abgezogen.

Die Nachweisgrenze für die Nitratreduktion ist im Verhältnis zur Präzision der ${ }^{15}\left(\mathrm{~N}_{2}+\mathrm{N}_{2} \mathrm{O}\right)$-Produktion relativ hoch. Das erklärt sich folgendermaßen: Zum einen handelt es sich um eine indirekte Messung: Die Nitratabbaurate ergibt sich aus der Differenz zwischen Zugabe und Wiederfindung vom Nitrat. Darüber hinaus beinhaltet die Versuchsordnung potentielle Fehlerquellen. Hier ist die begrenzte Genauigkeit bei der Quantifizierung der Verschleppung von Nitrat zwischen aufeinanderfolgenden Inkubationsintervallen zu nennen. Von einigen Autoren wird auch eine Nitratsorption an Tonmineralien wie Montmorilloniten postuliert (KöLLE, 1999). Bei Auftreten einer Nitratsorption würde die Denitrifikation durch die Differenzmethode überschätzt werden. Bei der Auswertung der Nitratdifferenzen fiel auf, dass praktisch keine Nullwerte auftraten. Auch in Proben, die fast frei von Reduktionsmitteln waren, wurde eine unvollständige Nitratwiederfindung erzielt. Messungen $\operatorname{der}^{15}\left(\mathrm{~N}_{2}+\mathrm{N}_{2} \mathrm{O}\right)$-Bildung die in einigen dieser Proben durchgeführt wurden, ergaben jedoch Nullwerte. Daraus wurde gefolgert, dass die unvollständige Nitratwiederfindung dieser Proben nicht durch Denitrifikation sondern ausschließlich durch methodische Ungenauigkeit und/oder Nitratsorption zu erklären ist. Zur Abschätzung der Denitrifikation aus den Nitratdifferenzen wurden des- 
halb die Nitrat-Differenzen dieser "Nullproben" als Blindwerte betrachtet. Die Denitrifikationsraten wurden nach folgender Beziehung berechnet:

$\mathrm{DR}\left(\mathrm{mg} \mathrm{N} \mathrm{kg}^{-1} \mathrm{~d}^{-1}\right)=\frac{\left[\mathrm{d} \mathrm{NO}_{3}(\text { Probe })\left(\mathrm{mg} \mathrm{N} \mathrm{kg}^{-1}\right)\right]}{\text { Inkubationsdauer }(\mathrm{d})}-\left[\mathrm{d} \mathrm{NO}_{3}(\right.$ Blindwert $\left.\left.) \mathrm{mg} \mathrm{N} \mathrm{kg}^{-1} \mathrm{~d}^{-1}\right)\right]$

$\mathrm{DR}=$ Denitrifikationsrate

$\mathrm{d} \mathrm{NO}_{3}=$ Nitratdifferenz zwischen Beginn und Ende eines Inkubationsintervalls

Die Nachweisgrenze für die Raten der Nitratreduktion betrug zwischen $0,04 \mathrm{mg} \mathrm{N} \mathrm{kg}^{-1}$ $\mathrm{d}^{-1}$ (Inkubationsintervall von 200 Tagen) und $0,29 \mathrm{mg} \mathrm{N} \mathrm{kg}^{-1} \mathrm{~d}^{-1}$ ( Inkubationsintervall von 30 Tagen).

Anhand der Ergebnisse können die Verläufe der Nitrat-Reduktion in drei Gruppen unterteilt werden: 1. Böden, bei denen der Verlauf zu Beginn mit einer sehr hohen NitratReduktionsrate zu kennzeichnen ist (Aher-Kämpe, Reinshof und Schladen) und bei denen die Raten nach ca. 200 Tagen auf konstantem Niveau liegen. 2. Böden, deren Anfangsrate sich im mittleren Bereich befindet (z.B. Dinklage) und bei denen nach kurzer Zeit (nach ca. 100 Tagen) ein deutlicher Rückgang der Reduktion erfolgt. 3. Böden, die eine niedrige Anfangsrate aufweisen (Bühren, Fuhrberg, Freistatt und Vechtel) und bei denen die Raten nach kurzer Zeit auf ein sehr niedriges Niveau zurückgehen.

Die höchste Abbaurate von 2,5 $\mathrm{mg} \mathrm{kg}^{-1} \mathrm{~d}^{-1}$ zeigt sich im Profil Reinshof (Schicht 4, Tiefe 180-200 cm). Dies ist auf den hohen Reduktionsmittel-Vorrat (vgl. Abb. 4 Tiefenverteilung) zurückzuführen.

Bei allen Proben sind die Nitratreduktionsraten zu Beginn am höchsten. Die oberen Messtiefen (oberhalb des Grundwasserspiegels) weisen häufig vergleichsweise geringe Abbauraten auf (z. B. Schladen, Schicht 1, 160-180cm Tiefe). Ein Anstieg der Raten ist häufig mit zunehmender Tiefe aufgrund steigender Reduktionsmittel-Vorräte (vgl. Abb. 4 Tiefenverteilung) zu beobachten. Z. B. weist der Standort Schladen bei einer Tiefe von 180-240 cm eine Nitratreduktionsrate von $0,9 \mathrm{mg} \mathrm{kg}^{-1} \mathrm{~d}^{-1}$ und bei einer Tiefe von 240 - $340 \mathrm{~cm}$ eine Rate von 2,2 $\mathrm{mg} \mathrm{kg}^{-1} \mathrm{~d}^{-1}$ auf (Abb. 5 Schladen, Schicht 2 und 3). Die

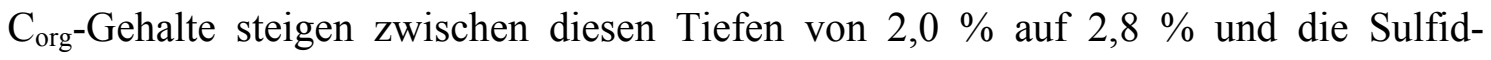
Gehalte von $85 \mathrm{mg} \mathrm{kg}^{-1}$ auf $664 \mathrm{mg} \mathrm{kg}^{-1}$.

Nach anfänglichem Rückgang der Nitratabbaurate zeigt sich bei Aher-Kämpe und in Schladen nach 250 Tagen und bei Reinshof nach 450 Tagen eine Stabilisierung (Abb. $5)$. 

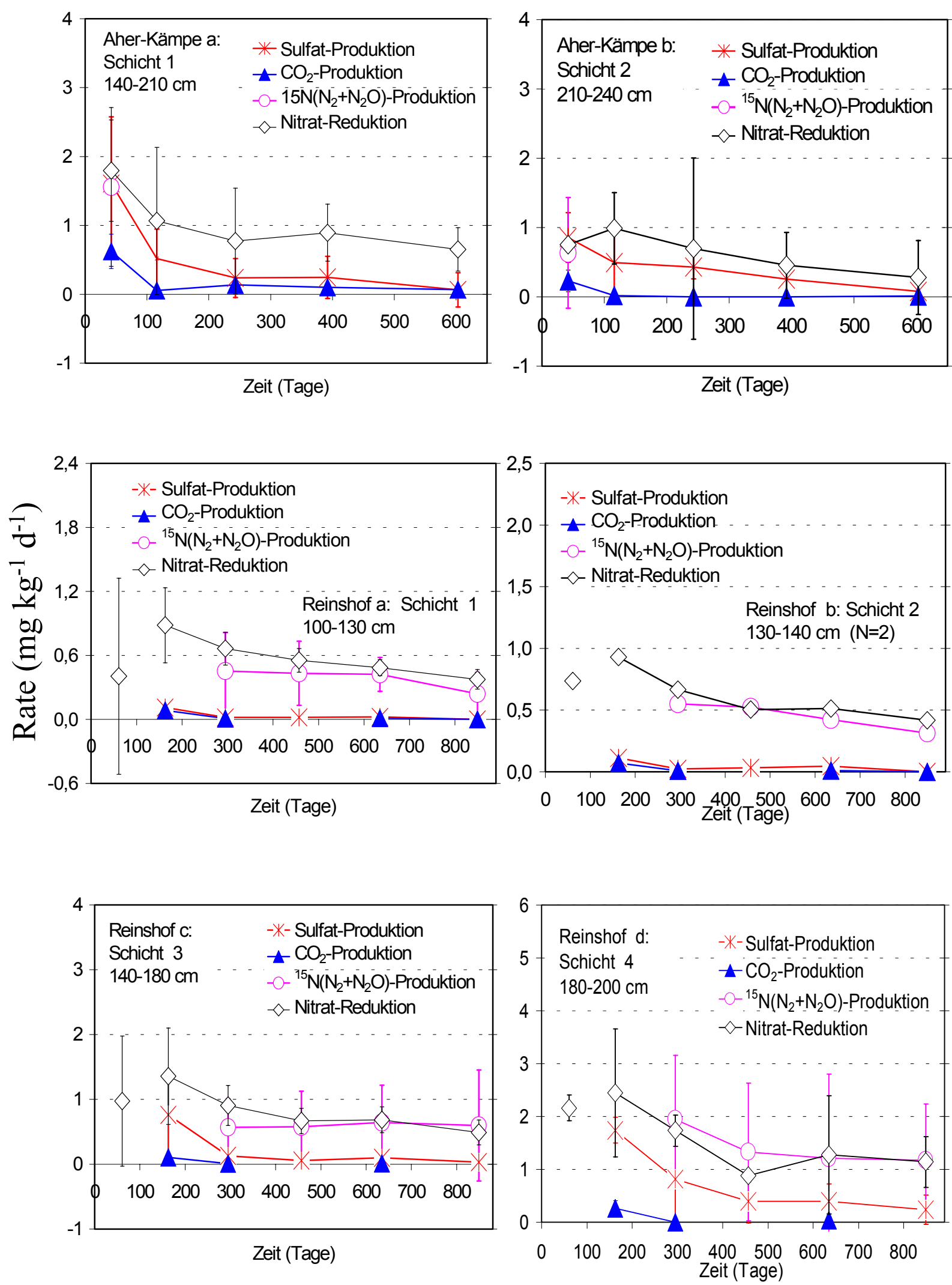

Abb. 5: Anaerobe Inkubation: Zeitverläufe für Nitratreduktion ( $\left.\mathrm{mg} \mathrm{N} \mathrm{kg}^{-1} \mathrm{~d}^{-1}\right)$, Sulfatproduktion ( $\left.\mathrm{mg} \mathrm{S} \mathrm{kg}^{-1} \mathrm{~d}^{-1}\right), \mathrm{CO}_{2}$-Produktion $\left(\mathrm{mg} \mathrm{C} \mathrm{kg}^{-1} \mathrm{~d}^{-1}\right)$ und ${ }^{15}\left(\mathrm{~N}_{2}+\mathrm{N}_{2} \mathrm{O}\right)$-Produktion $\left(\mathrm{mg} \mathrm{N} \mathrm{kg}^{-1} \mathrm{~d}^{-1}\right)$. Messpunkte gelten jeweils für das vorangegangene Zeitintervall 

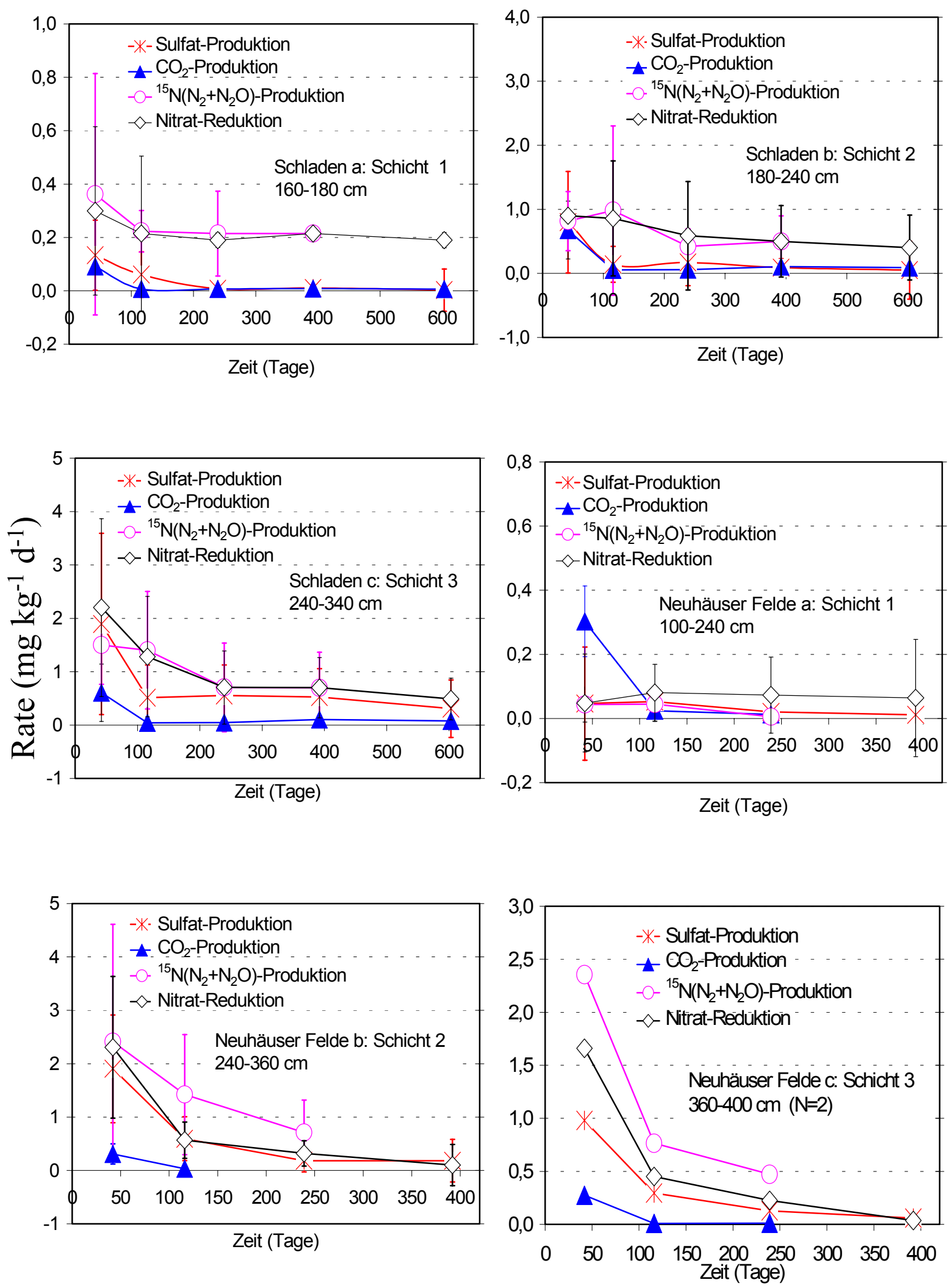

Abb. 5 (Fortsetzung): Anaerobe Inkubation: Zeitverläufe für Nitratreduktion $\left(\mathrm{mg} \mathrm{N} \mathrm{kg}^{-1} \mathrm{~d}^{-1}\right)$, Sulfatproduktion $\left(\mathrm{mg} \mathrm{S} \mathrm{kg}^{-1} \mathrm{~d}^{-1}\right)$, $\mathrm{CO}_{2}$-Produktion (mg $\left.\mathrm{C} \mathrm{kg}^{-1} \mathrm{~d}^{-1}\right)$ und ${ }^{15}\left(\mathrm{~N}_{2}+\mathrm{N}_{2} \mathrm{O}\right)$-Produktion $\left(\mathrm{mg} \mathrm{N} \mathrm{kg}^{-1} \mathrm{~d}^{-1}\right)$. Messpunkte gelten jeweils für das vorangegangene Zeitintervall 

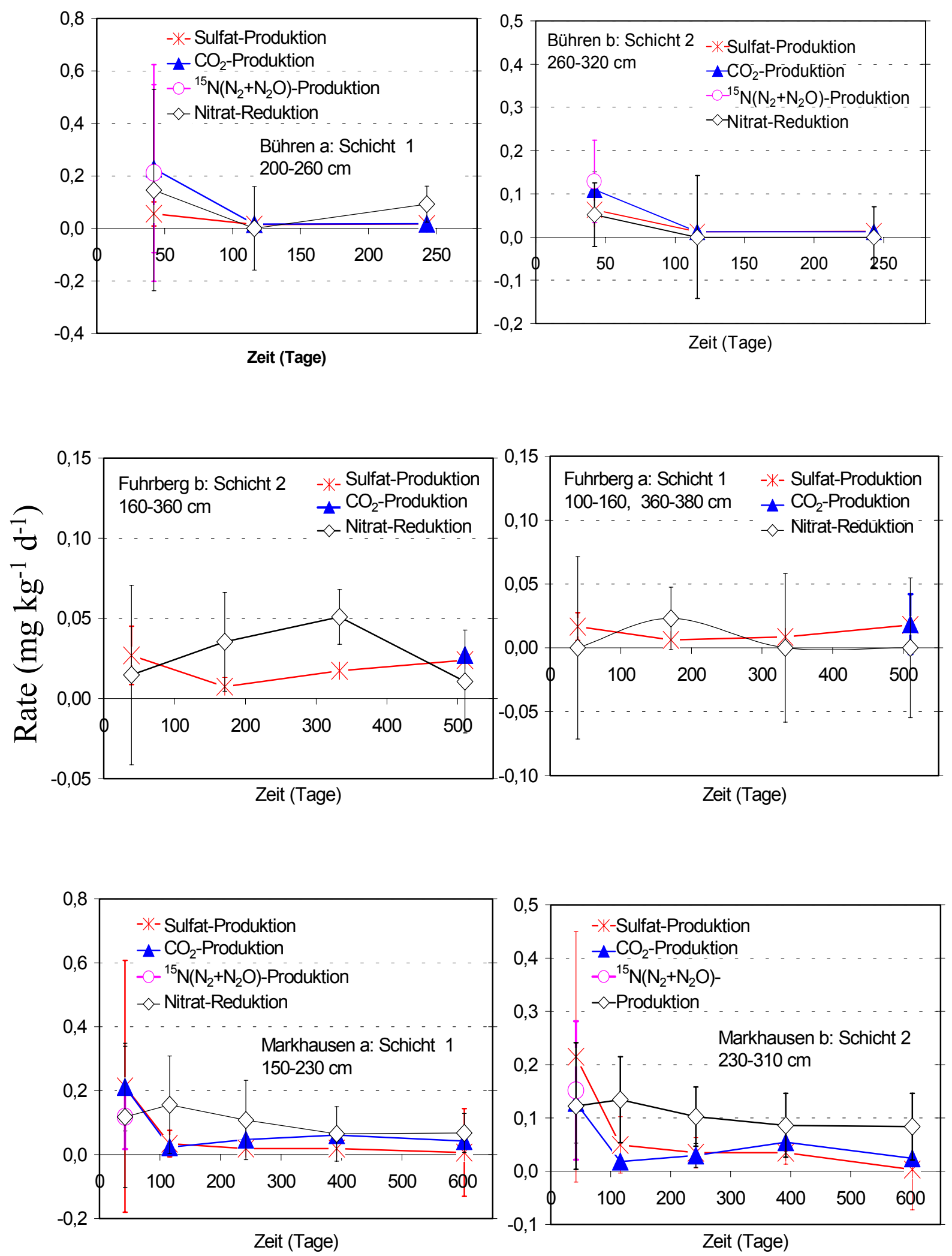

Abb. 5 (Fortsetzung): Anaerobe Inkubation: Zeitverläufe für Nitratreduktion ( $\mathrm{mg} \mathrm{N} \mathrm{kg}^{-1} \mathrm{~d}^{-1}$ ), Sulfatproduktion ( $\mathrm{mg} \mathrm{S} \mathrm{kg}^{-1} \mathrm{~d}^{-1}$ ), $\mathrm{CO}_{2}$-Produktion ( $\mathrm{mg}$ $\left.\mathrm{C} \mathrm{kg}^{-1} \mathrm{~d}^{-1}\right)$ und ${ }^{15}\left(\mathrm{~N}_{2}+\mathrm{N}_{2} \mathrm{O}\right)$-Produktion $\left(\mathrm{mg} \mathrm{N} \mathrm{kg}^{-1} \mathrm{~d}^{-1}\right)$. Messpunkte gelten jeweils für das vorangegangene Zeitintervall 

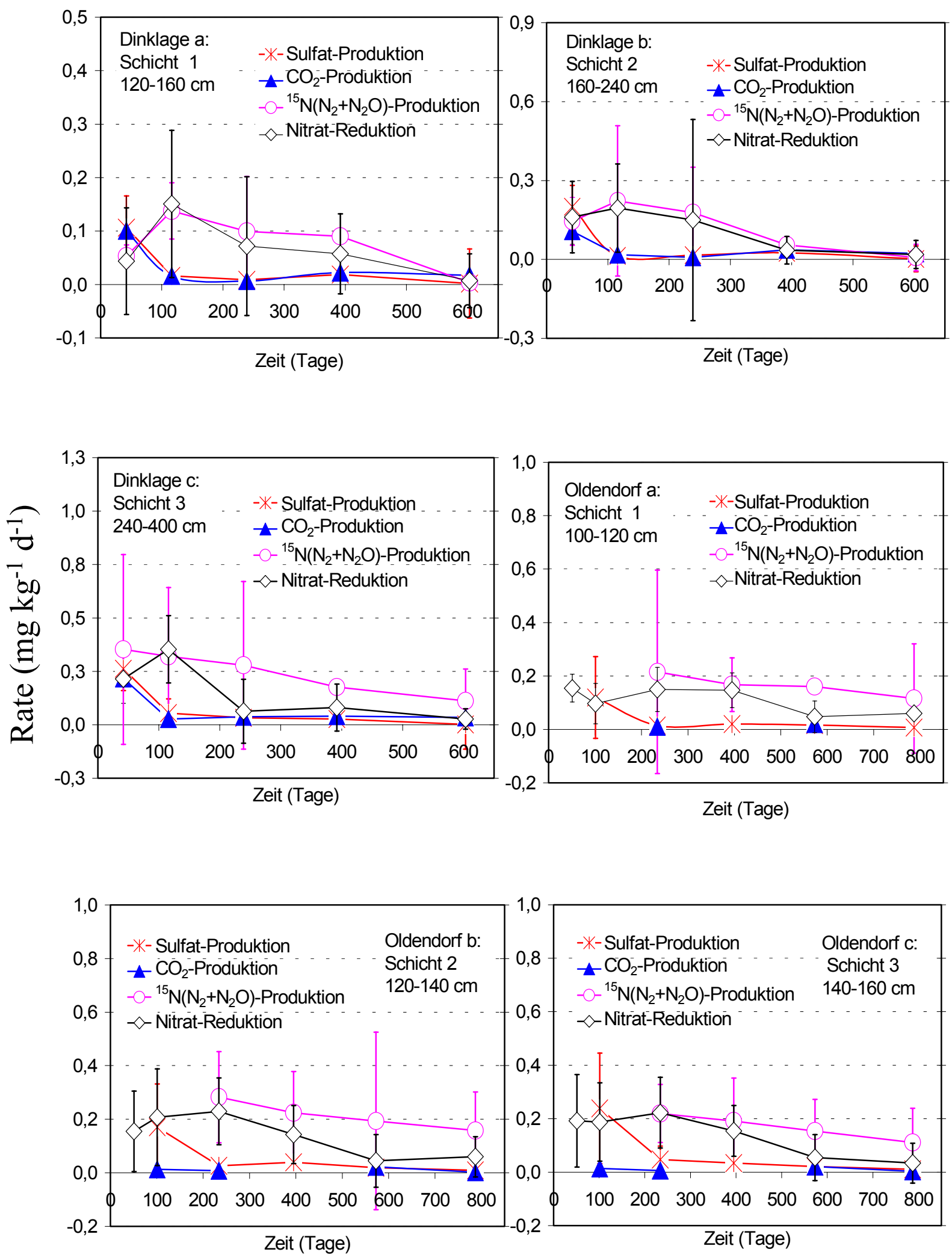

Abb. 5 (Fortsetzung): Anaerobe Inkubation: Zeitverläufe für Nitratreduktion $\left(\mathrm{mg} \mathrm{N} \mathrm{kg}^{-1} \mathrm{~d}^{-1}\right)$, Sulfatproduktion $\left(\mathrm{mg} \mathrm{S} \mathrm{kg}^{-1} \mathrm{~d}^{-1}\right), \mathrm{CO}_{2}$-Produktion (mg $\left.\mathrm{C} \mathrm{kg}^{-1} \mathrm{~d}^{-1}\right)$ und ${ }^{15}\left(\mathrm{~N}_{2}+\mathrm{N}_{2} \mathrm{O}\right)$-Produktion $\left(\mathrm{mg} \mathrm{N} \mathrm{kg}^{-1} \mathrm{~d}^{-1}\right)$. Messpunkte gelten jeweils für das vorangegangene Zeitintervall 

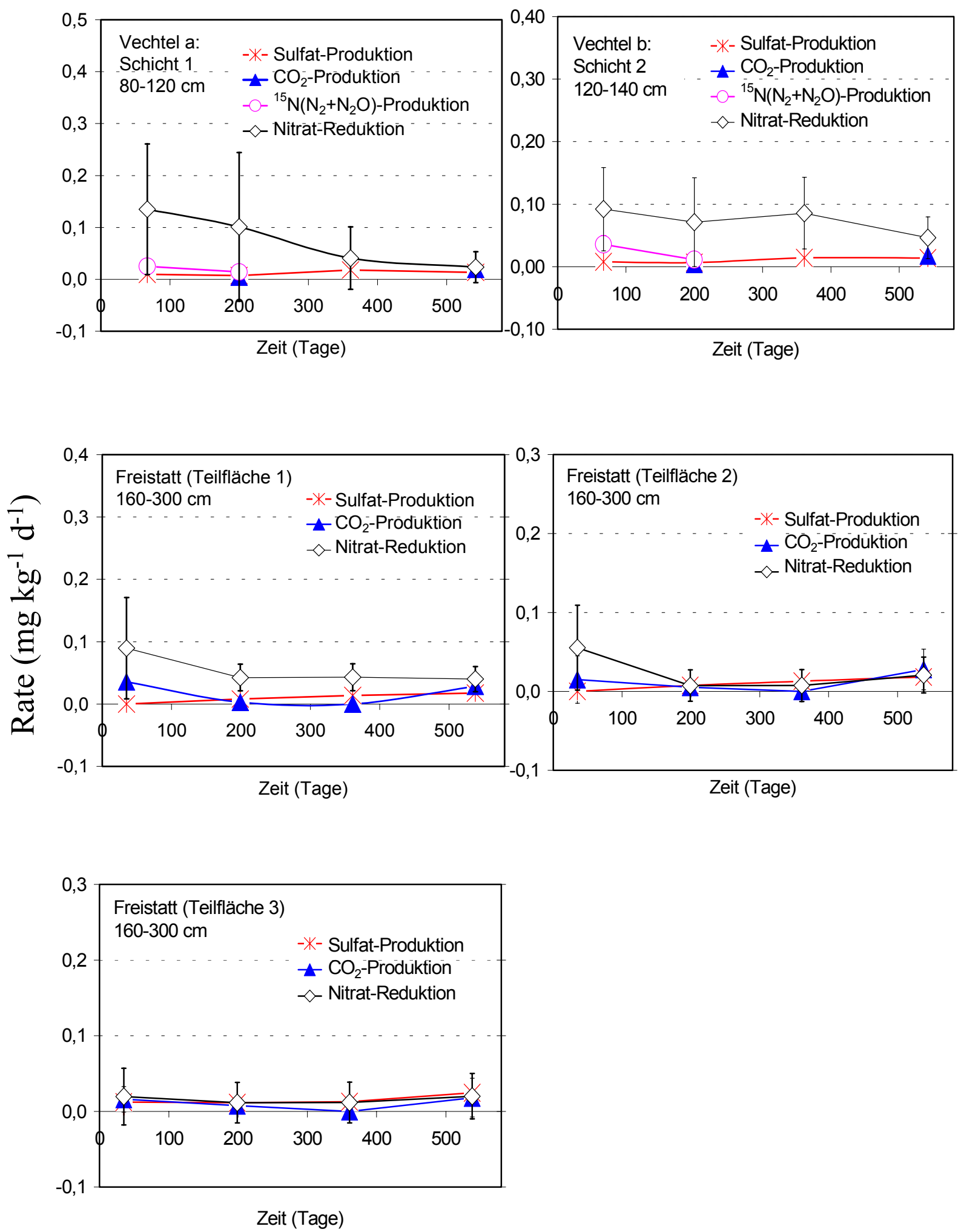

Abb. 5 (Fortsetzung): Anaerobe Inkubation: Zeitverläufe für Nitratreduktion $\left(\mathrm{mg} \mathrm{N} \mathrm{kg}^{-1} \mathrm{~d}^{-1}\right)$, Sulfatproduktion ( $\left.\mathrm{mg} \mathrm{S} \mathrm{kg}^{-1} \mathrm{~d}^{-1}\right), \mathrm{CO}_{2}$-Produktion (mg $\left.\mathrm{C} \mathrm{kg}^{-1} \mathrm{~d}^{-1}\right)$ und ${ }^{15}\left(\mathrm{~N}_{2}+\mathrm{N}_{2} \mathrm{O}\right)$-Produktion $\left(\mathrm{mg} \mathrm{N} \mathrm{kg}^{-1} \mathrm{~d}^{-1}\right)$. Messpunkte gelten jeweils für das vorangegangene Zeitintervall 
Im Profil Neuhäuser-Felde ist schon ab 100 Tagen ein deutlicher Rückgang der Abbaurate zu erkennen.

Anhand der Abb. 5 wird deutlich, dass bei allen Lehm-Standorten mit Ausnahme von Bühren (geringer Reduktionsmittelvorrat, s. Abb. 4) die Nitratreduktionsrate bis zum Versuchsende nicht vollständig zum Erliegen kommt.

Eine ähnliche Dynamik der zeitlichen Denitrifikationsverluste zeigen die Sand-Böden, jedoch auf einem niedrigeren Niveau (Abb. 5). Die höchste Nitratverlustrate von 0,4 mg $\mathrm{kg}^{-1} \mathrm{~d}^{-1} \mathrm{zu}$ Beginn tritt bei Dinklage auf (Schicht 3, Tiefe 240-400 cm), wobei der mittlere $\mathrm{C}_{\text {org-Gehalt }}$, $16 \mathrm{mg} \mathrm{kg}^{-1}$ (s. Abb. 4) beträgt. Hier ist die Abbaurate nach 600 Tagen vollständig zum Erliegen gekommen. Dies trifft für alle Sand-Böden zu: z. B. wurde nach 500 (Fuhrberg), 600 (Dinklage, Markhausen) und 800 Tagen (Oldendorf) keine Nitrat-Reduktion mehr festgestellt.

Für die Bestimmung der autotrophen Denitrifikation aus reduzierten mineralischen Schwefelverbindungen wurde bei den Inkubationsversuchen die produzierte Menge an Sulfat bestimmt. Die in der Abb. 5 dargestellten Zeitintervalle der SulfatProduktionsrate zeigen, dass die Sulfat-Produktionsrate, genau wie die NitratReduktion, zu Beginn viel höher liegt und in den folgenden Intervallen erheblich abnimmt. Die stärkste Produktionsrate von $1,9 \mathrm{mg} \mathrm{kg}^{-1} \mathrm{~d}^{-1}$ wurde $\mathrm{zu}$ Beginn bei Aher Kämpe (Abb. 5, Schicht 1, Tiefe 140-210 cm) festgestellt. Nach 100 Tagen hat die Rate drastisch abgenommen.$\left(0,5 \mathrm{mg} \mathrm{kg}^{-1} \mathrm{~d}^{-1}\right)$ und bleibt danach bis zum Versuchsende mehr oder weniger konstant (Abb. 5 Schicht 1 und 2). Bei fast allen sulfidhaltigen Proben war eine im Vergleich zur Nitratreduktionsrate schnellere Abnahme der Sulfatproduktionsrate zu beobachten. Offenbar handelt es sich hier um leicht oxidierbare Sulfide. Eine bedeutende autotrophe Denitrifikation wurde bei allen Lehm-Böden mit Ausnahme von Bühren festgestellt.

Die hohe Sulfatbildung in einigen der unteren Messtiefen spiegelt die hohen Sulfidgehalte (vgl. Abb. 4) in den jeweiligen Tiefen wider.

Die sulfidarmen Böden weisen Sulfatproduktionraten im Bereich der Nachweisgrenze auf.

Da die indirekte Bestimmung der denitrifikativen N-Verluste aus dem Nitratabbau mit einigen methodischen Fehlern behaftet ist (s.o.), wurden zur besseren Erfassung der Denitrifikation die gasförmigen Produkte mittels ${ }^{15} \mathrm{~N}$-Tracertechnik gemessen. 
Die Ergebnisse zeigen eine im Zeitverlauf ähnliche Dynamik (Abb. 5). Die Größenordnung der direkten Messungen $\left({ }^{15}\left(\mathrm{~N}_{2}+\mathrm{N}_{2} \mathrm{O}\right)\right.$-Produktion) stimmt gut mit der indirekten Methode (Nitratabbau) überein.

Aus Abb. 5 sind auch die $\mathrm{CO}_{2}$-Produktionsraten ersichtlich. Bei allen Proben zeigt sich ein einheitliches Muster: Im ersten Inkubationsintervall ist eine deutliche $\mathrm{CO}_{2}$ Produktion erkennbar. Unter Berücksichtigung der Stöchiometrie bei der heterotrophen Denitrifikation (s. Gl. Kap. 2.1); $\mathrm{CO}_{2}-\mathrm{C}: \mathrm{NO}_{3}-\mathrm{N}=0,93: 1$ ) lässt sich abschätzen, ob die Größenordnung dieser anfänglichen $\mathrm{CO}_{2}$-Produktion aus der heterotrophen Denitrifikation erklärt werden kann. Bei dieser Betrachtung wird angenommen, dass die autotrophe Denitrifikation von der Sulfatproduktion widergespiegelt wird und dass die heterotrophe Denitrifikation aus der Differenz zwischen Gesamt-Denitrifikation und autotropher Denitrifikation abgeleitet werden kann. Es zeigt sich, dass die heterotrophe Denitrifikation z.B. im Profil Schladen mit $0,90 \mathrm{mg} \mathrm{N} \mathrm{kg}^{-1} \mathrm{~d}^{-1}$ (Schicht 2, $180-240 \mathrm{~cm}$ ) und im Profil Dinklage (Schicht 2, $240-400 \mathrm{~cm}$ ) mit 0,16 mg N kg-1 $\mathrm{m}^{-1}$ größenordnungsmäßig den Werten der $\mathrm{CO}_{2}$-Produktion (Schladen 0,68 $\mathrm{mg} \mathrm{C} \mathrm{kg}^{-1} \mathrm{~d}^{-1}$; Dinklage $0,11 \mathrm{mg} \mathrm{C} \mathrm{kg} \mathrm{d}^{-1}$ ) entspricht. Nach dem ersten Inkubationsintervall fällt die $\mathrm{CO}_{2}$ Produktion bei allen Proben stark ab. Sie ist dann immer sehr viel kleiner als die heterotrophe Denitrifikation. Beispielsweise beträgt in Schladen die heterotrophe Denitrifikation (Schicht 2, 180-240 cm) zwischen 0,40 und 0,86 mg kg $\mathrm{m}^{-1}$ und in Dinklage (Schicht 2, 240-400 cm) zwischen $0,03 \mathrm{mg} \mathrm{kg}^{-1} \mathrm{~d}^{-1}$ und $0,35 \mathrm{mg} \mathrm{kg}^{-1} \mathrm{~d}^{-1}$, während die $\mathrm{CO}_{2}$-Produktion nur zwischen 0,05 (Schladen) und 0,01 (Dinklage) mg N-Äquivalent $\mathrm{kg}^{-1} \mathrm{~d}^{-1}$ liegt.

Es ist $\mathrm{zu}$ folgern, dass die freigesetzte $\mathrm{CO}_{2}$-Menge keine geeignete Messgröße für die Ermittlung der heterotrophen Denitrifikation ist.

Es lässt sich folgendes zusammenfassen:

- Böden mit hohen Reduktionsmittelgehalten im Untergrund weisen eine hohe Abbaurate zu Beginn auf, die sich dann im Laufe der Zeit verringert.

- Die Nitratreduktionsrate stabilisiert sich bis zum Versuchsende auf einem verminderten Niveau.

- In vielen Fällen erfolgt eine deutliche Zunahme der Abbaurate mit zunehmender Tiefe.

- Die Denitrifikationsrate in Böden mit geringen Reduktionsmittelgehalten nimmt mit der Dauer der Inkubationszeit drastisch ab und kommt zu einem mehr oder weniger vollständigen Erliegen. 
- Die indirekte Messung der Denitrifikation stimmt von der Größenordnung mit der direkten Messung der gasförmigen Denitifikationsprodukte überein.

- Die $\mathrm{CO}_{2}$-Freisetzung im Untergrund ist keine geeignete Größe für die Abschätzung der heterotrophen Denitrifikation in den untersuchten Böden.

- Die Nitrat-Reduktion und die Sulfat-Produktion nehmen wegen der begrenzten Verfügbarkeit der Reduktionsmittel mit zunehmender Dauer der Inkubation ab. Dies verdeutlicht eine beginnende Erschöpfung des Denitrifikationspotentials.

\subsection{Messergebnisse der aeroben Inkubationsversuche}

Zur Bestimmung der biologischen Verfügbarkeit der reduzierten Schwefelverbindungen in den untersuchten Böden wurden Langzeitinkubationsversuche unter anaeroben Bedingungen durchgeführt.

In Abb. 6 sind die zeitlichen Verläufe der aeroben Sulfatproduktion über ein bis zwei Jahre dargestellt. Zum Vergleich wird zusätzlich die Kinetik der anaeroben Sulfatproduktion gezeigt. Die ersten Produktionsraten unter aerobem Milieu liegen im Vergleich zur anaeroben Inkubation bei Böden mit hohen Sulfidgehalten deutlich höher. Die zweite Rate verhält sich auch bei den meisten Böden mit hohen Sulfidgehalten wie die erste Rate. Die folgenden Produktionsraten nehmen sprunghaft ab und ähneln den anaeroben Produktionsraten. Eine Ausnahme stellt die Schicht 3 des Profils NeuhäuserFelde (Tiefe 360 - $400 \mathrm{~cm}$ ) dar, bei der die dritte und vierte aerobe Sulfatproduktionsrate noch deutlich höher liegt als die anaerobe Sulfatproduktionsrate. In dieser Schicht ist die Anfangsrate von $6,30 \mathrm{mg} \mathrm{kg}^{-1} \mathrm{~d}^{-1}$ insgesamt auch am höchsten.

Die zeitlichen Verläufe der Sulfatproduktion verhalten sich zwischen 100 und 600 Tagen mehr oder weniger konstant auf geringem Niveau. Nach 600 bis 700 Tagen ist die Sulfatbildung fast bei allen Proben zum Erliegen gekommen.

Beide Produktionskinetiken, sowohl die aerobe als auch die anaerobe, verhalten sich sehr ähnlich, indem sie eine hohe Anfangsumsetzungsrate aufweisen und die darauf folgenden Raten sprunghaft abnehmen. Der Vergleich zwischen den unterschiedlichen Schichten zeigt, dass die Sulfatproduktion in der Regel mit zunehmender Bodentiefe steigt. Dies ist mit der Tiefenverteilung der Sulfidgehalte zu erklären (vgl. Abb.4). 

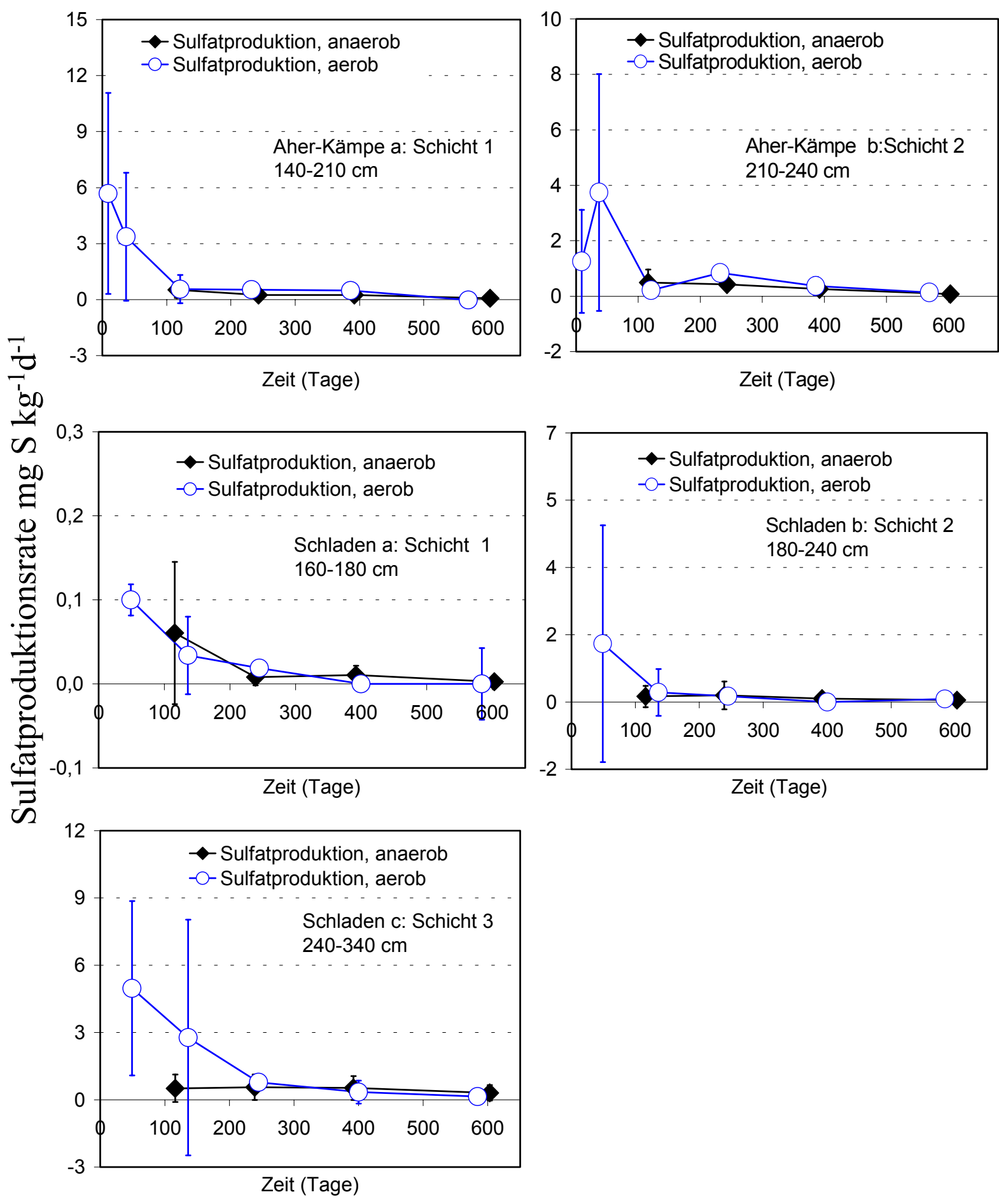

Abb. 6: Zeitverläufe der anaeroben und aeroben Sulfatproduktion. Messpunkte gelten jeweils für das vorangegangene Zeitintervall 

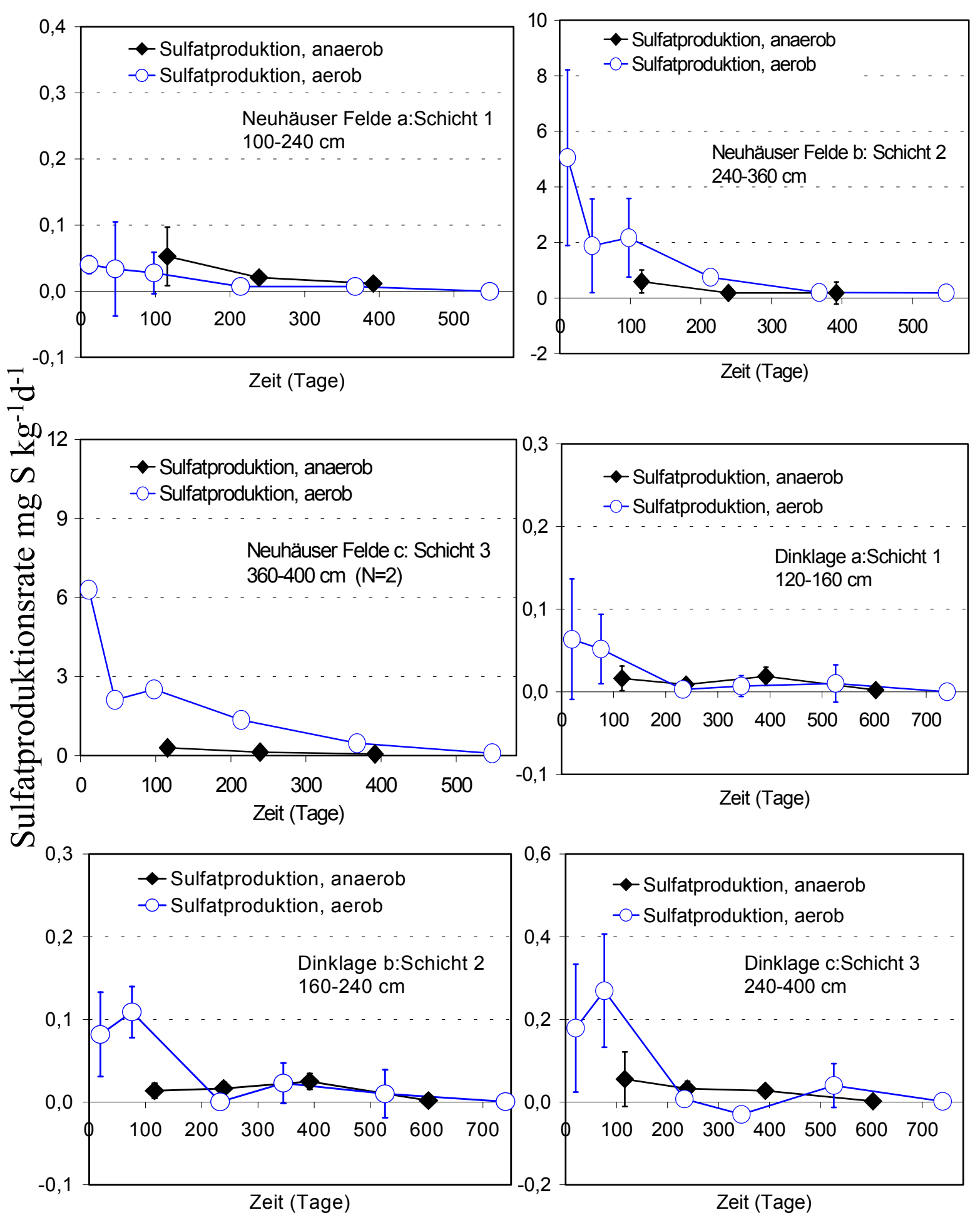

Abb. 6 (Fortsetzung): Zeitverläufe der anaeroben und aeroben Sulfatproduktion. Messpunkte gelten jeweils für das vorangegangene Zeitintervall. 

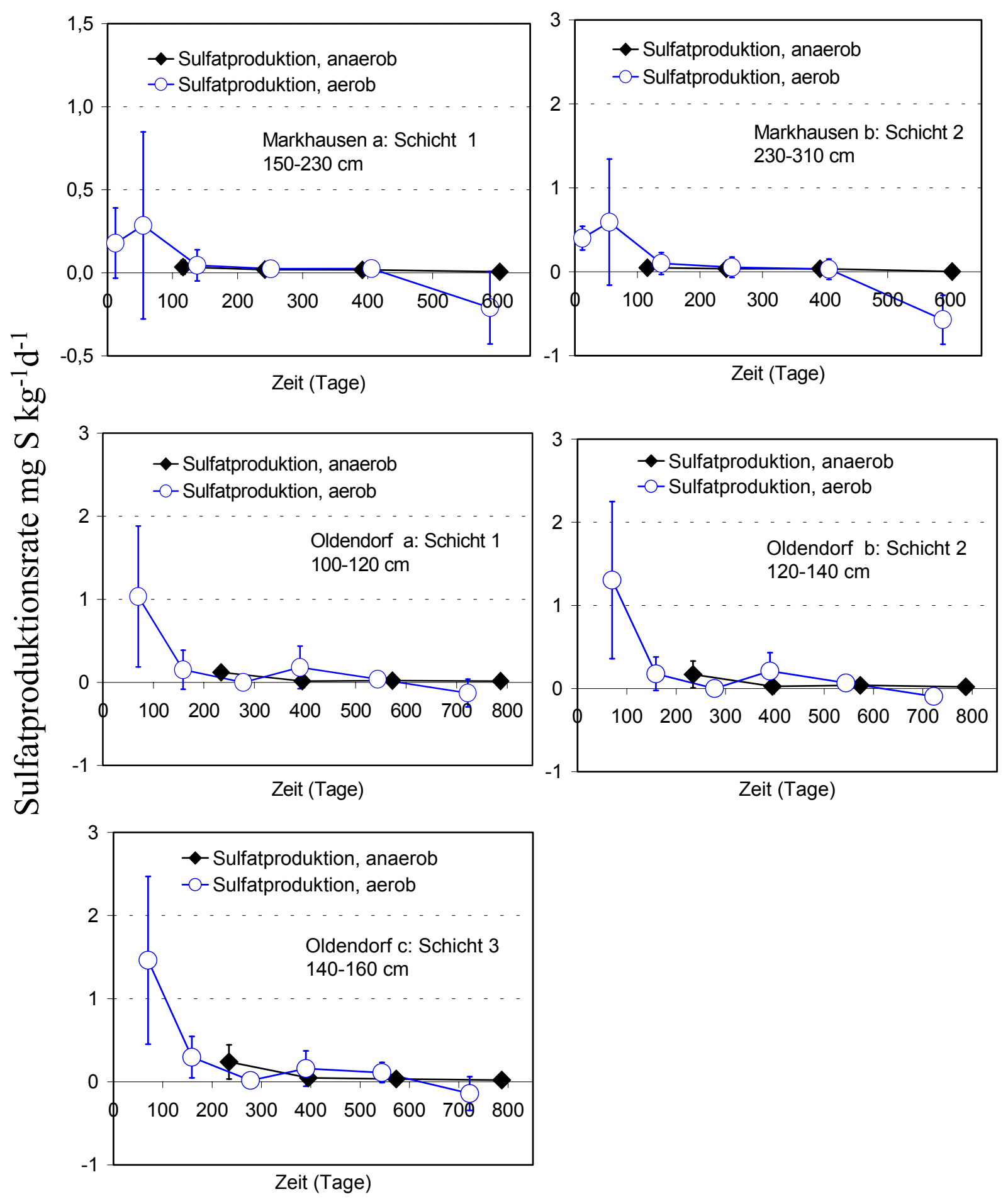

Abb. 6 (Fortsetzung): Zeitverläufe der anaeroben und aeroben Sulfatproduktion. Messpunkte gelten jeweils für das vorangegangene Zeitintervall. 


\subsection{Einfluss der Temperatur auf die Nitratreduktion und Sulfatproduktion}

Die Versuche zur Temperaturabhängigkeit auf die Umsetzungsraten erfolgten an einigen ausgewählten Standorten. Hierzu wurden die Proben bei zwei Temperaturstufen $\left(10^{\circ} \mathrm{C}\right.$ und $\left.25^{\circ} \mathrm{C}\right)$ inkubiert. An allen untersuchten Proben ist ein Temperatureinfluss auf die Nitratreduktion und Sulfatproduktion zu erkennen (Abb. 7). Bei einer Temperatur von $10^{\circ} \mathrm{C}$ wurde gegenüber $25^{\circ} \mathrm{C}$ ein deutlich geringerer Denitrifikationsverlust beobachtet.

In der Regel liegen auch die Sulfatproduktionen bei $25^{\circ} \mathrm{C}$ höher als bei $10^{\circ} \mathrm{C}$. Bei den Schichten mit geringerem Aktivitätsniveau gibt es z. T. Abweichungen von dieser Regel. Dies ist mit begrenzter Messempfindlichkeit sowie mit der Heterogenität des Probenmaterials zu erklären. Bei den Schichten mit den höheren Aktivitäten ist jedoch klar erkennbar, dass die Sulfatproduktionen bei $25^{\circ} \mathrm{C}$ deutlich höher liegen.

Tab. 7 zeigt die Verhältnisse zwischen den Temperaturstufen für die Nitratreduktion und die Sulfatproduktion.

Tab. 7: Temperatureinfluss auf die Nitratreduktion und die Sulfatproduktion: Verhältnis der Temperaturstufen $\left(25^{\circ} \mathrm{C} / 10^{\circ} \mathrm{C}\right)$

\begin{tabular}{lllllll}
\hline Standort & $\begin{array}{l}\mathbf{N O}_{\mathbf{3}} \text { Reduktion } \\
\left(\mathbf{2 5}^{\circ} \mathbf{C} / \mathbf{1 0}^{\circ} \mathbf{C}\right)\end{array}$ & $\mathbf{Q}_{\mathbf{1 0}}$ & $\mathbf{N}$ & $\begin{array}{l}\mathbf{S O}_{4} \text {-Produktion } \\
\left(\mathbf{2 5}^{\circ} \mathbf{C} / \mathbf{1 0}\right.\end{array}$ & $\mathbf{Q}_{\mathbf{1 0}} \mathbf{C}$ & $\mathbf{N}$ \\
\hline Reinshof & $1,97 \pm 0,80$ & 1,64 & 7 & $2,37 \pm 0,99$ & 1,91 & 19 \\
Oldendorf & $1,48 \pm 0,33$ & 1,32 & 3 & $2,43 \pm 0,37$ & 1,95 & 3 \\
Vechtel & $2,44 \pm 0,93$ & 1,96 & 7 & $1,65 \pm 0,69$ & 1,43 & 7 \\
Dinklage & $2,03 \pm 1,02$ & 1,68 & 8 & $1,91 \pm 0,30$ & 1,61 & 12 \\
Markhausen & $3,22 \pm 1,48$ & 2,48 & 5 & $2,35 \pm 0,83$ & 1,90 & 3 \\
Aher Kämpe & $2,39 \pm 0,84$ & 1,93 & 3 & $2,27 \pm 0,60$ & 1,85 & 5 \\
Schladen & $2,04 \pm 0,33$ & 1,69 & 4 & $2,15 \pm 0,77$ & 1,77 & 6 \\
\hline
\end{tabular}

$\mathrm{N}: \quad$ Anzahl der Proben

$\mathrm{Q}_{10}$ : $\quad$ Faktor für die Zunahme der Reduktionsrate bei einem Temperaturanstieg von $10^{\circ} \mathrm{C}$ 

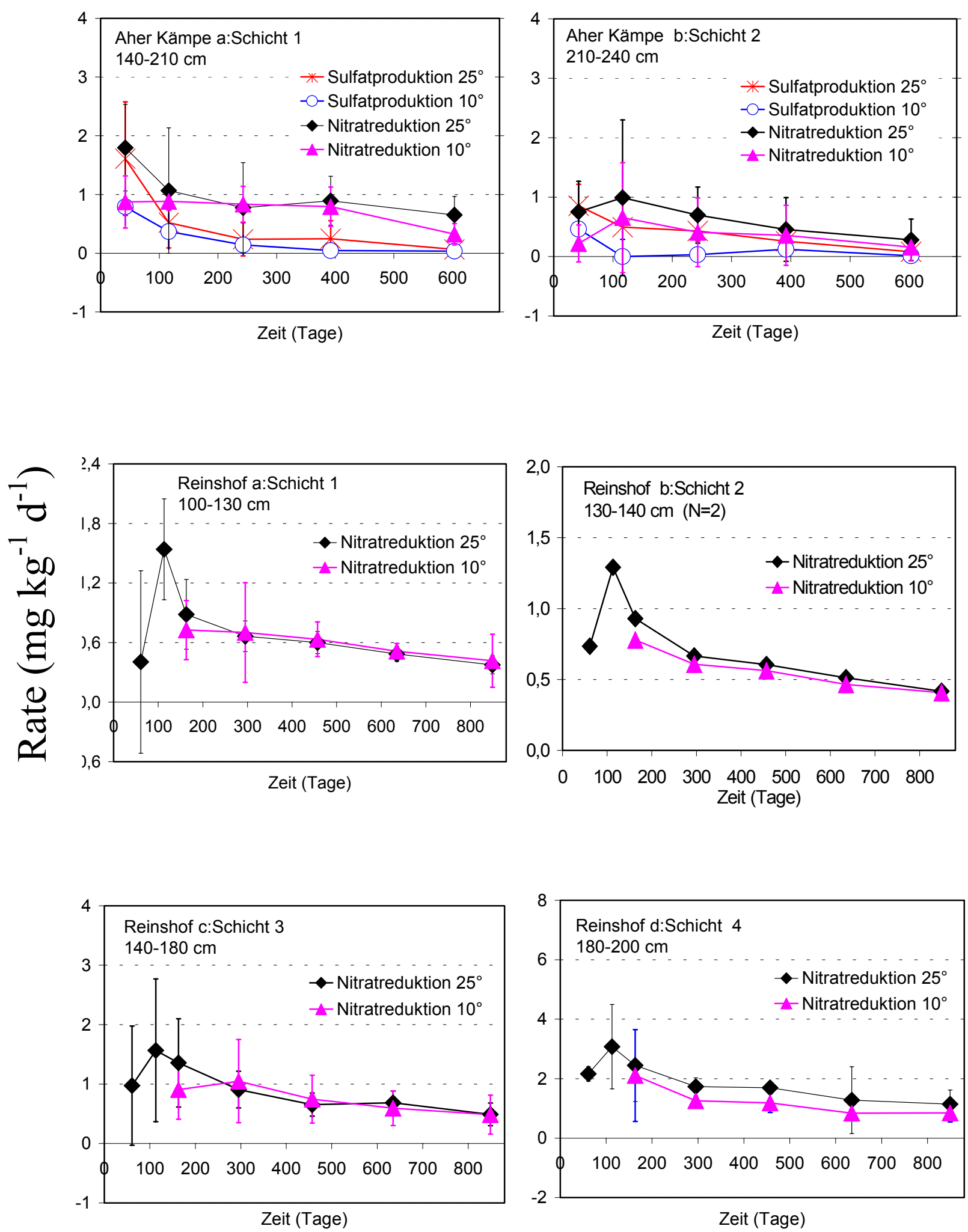

\section{Abb. 7: Zeitverläufe der unterschiedlichen Temperaturen $\left(10^{\circ} \mathrm{C}, 25^{\circ} \mathrm{C}\right)$ für die Ni- tratreduktion und Sulfatproduktion}



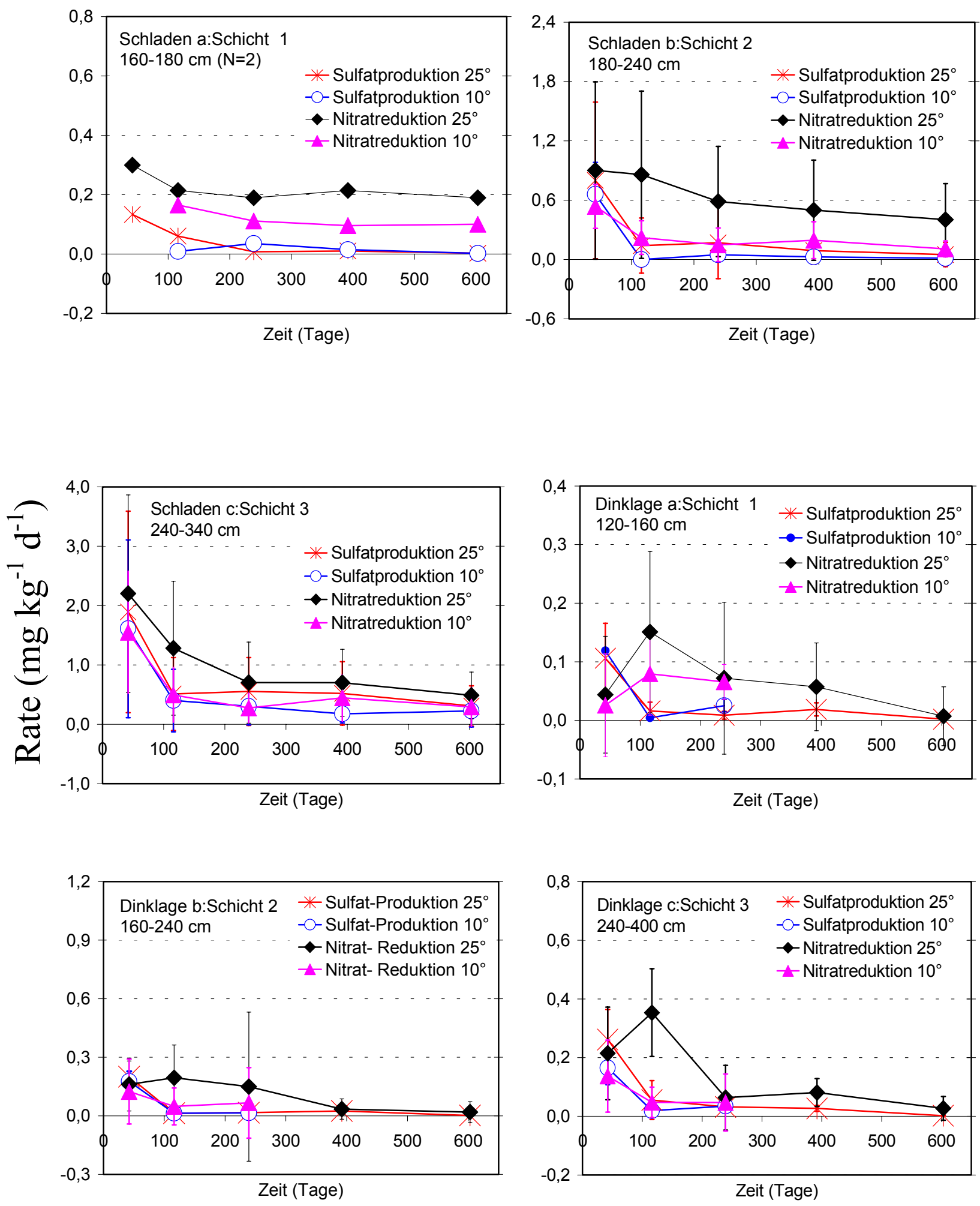

Abb. 7 (Fortsetzung): Zeitverläufe der unterschiedlichen Temperaturen $\left(10^{\circ} \mathrm{C}, 2^{\circ} \mathrm{C}\right)$ für die Nitratreduktion und Sulfatproduktion 

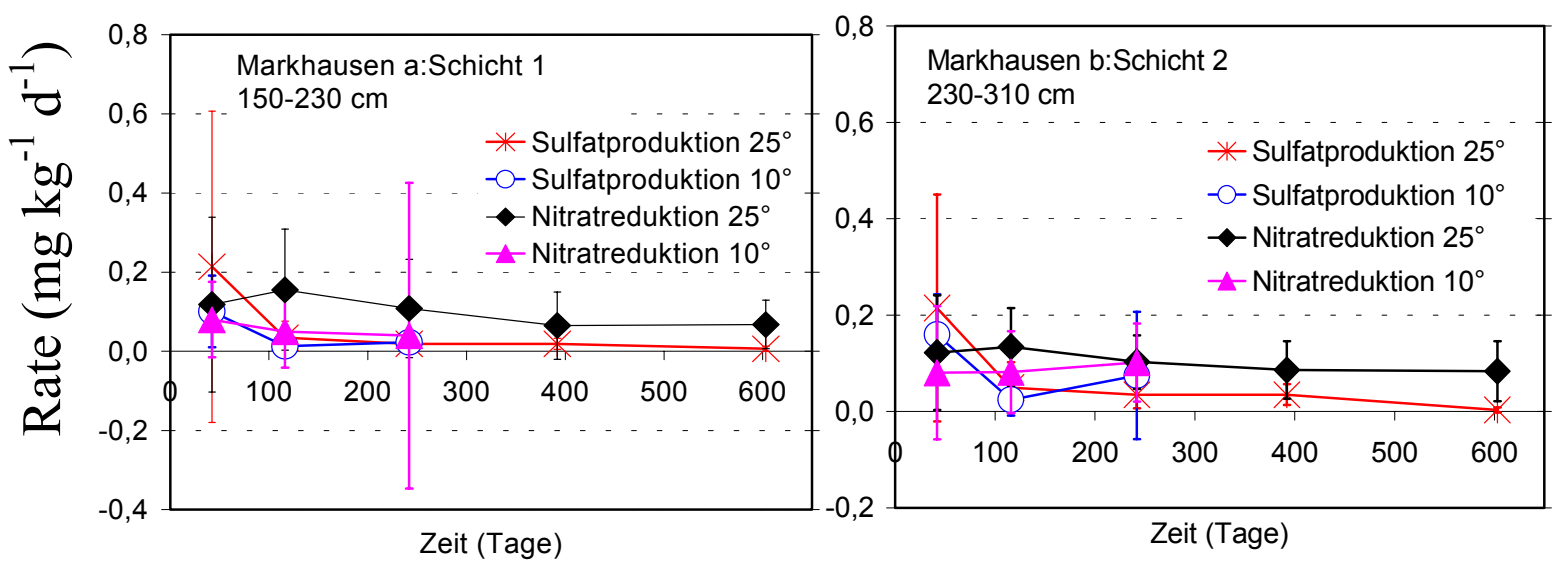

\section{Abb. 7 (Fortsetzung): Zeitverläufe der unterschiedlichen Temperaturen $\left(10^{\circ} \mathrm{C}\right.$, $25^{\circ} \mathrm{C}$ ) für die Nitratreduktion und Sulfatproduktion}

Aus Tab. 7 ist ersichtlich, dass die Denitrifikationsraten bei einem Temperaturanstieg um $15^{\circ} \mathrm{C}$ etwa um den Faktor 2 zu nehmen. Im Mittel ergibt sich über den betrachteten Temperaturbereich für die Nitratreduktion ein $\mathrm{Q}_{10}$-Wert (Faktor für die Zunahme der Reaktionsrate bei einem Temperaturanstieg von $10^{\circ} \mathrm{C}$ ) zwischen 1,32 und 2,48 und für die Sulfatproduktion ein $\mathrm{Q}_{10}$-Wert zwischen 1,43 und 1,95. Damit liegen die gemessenen $\mathrm{Q}_{10}$-Werte in dem Bereich, der in der Regel für mikrobielle Umsetzungen angegeben wird (z.B. STANFORD et al., 1975; LIND, 1980).

\subsection{Flächenmittelwerte der verschiedenen Aktivitäts-Kenngrößen}

Tab. 8 gibt einen Überblick über die Ergebnisse zur Reduktion von Nitrat und Produktion von Sulfat aus den anaeroben und aeroben Inkubationsversuchen. Dargestellt sind die Flächenmittelwerte der Umsatzraten zu Versuchsbeginn $\left(\mathrm{R}_{\mathrm{NO} 3}, \mathrm{DR}^{15}\left(\mathrm{~N}_{2}+\mathrm{N}_{2} \mathrm{O}\right)\right.$, $\left.\mathrm{R}_{\mathrm{SO} 4}\right)$, kumulierte Umsetzmengen $\left(\mathrm{M}_{\mathrm{NO} 3}, \mathrm{M}_{\mathrm{SO} 4}\right)$, die berechnete kumulierte Menge der heterotrophen Denitrifikation $\left(\mathrm{M}_{\mathrm{NO} 3} \mathrm{H}\right)$, das Verhältnis der heterotrophen Denitrifikation zur Gesamtumsatzrate $\left(\mathrm{M}_{\mathrm{NO} 3} \mathrm{H}: \mathrm{M}_{\mathrm{NO} 3 \text { (ges.) }}\right)$ sowie Indizes für die Nachhaltigkeit der Denitrifikation und die Verfügbarkeit der Reduktionsmittelvorräte. Die Schichtmittelwerte dieser Kenngroßen finden sich im Anhang (Tab. 4)

$\mathrm{R}_{\mathrm{NO} 3}$ ist die mittlere Nitratreduktionsrate des ersten Inkubationsintervalls. $\mathrm{DR}^{15}\left(\mathrm{~N}_{2}+\mathrm{N}_{2} \mathrm{O}\right)$ ist die ${ }^{15} \mathrm{~N}-\mathrm{Nitratreduktionsrate}$ am Ende des 1. Inkubationsintervalls. In 
den meisten Fällen stimmen beide Werte recht gut überein. Unterschiede sind mit der Messungenauigkeit bei der Nitratreduktion in den Böden geringer Aktivität sowie durch mögliche Änderungen der Umsatzrate während des ersten Inkubationsintervalls zu erklären.

Die höchsten $\mathrm{R}_{\mathrm{NO} 3}$ betragen zwischen 1 und 1,56 $\mathrm{mg} \mathrm{kg}^{-1} \mathrm{~d}^{-1}$ und sind bei den Böden mit hohen Reduktionsmittelvorräten zu beobachten (Aher Kämpe, Reinshof, Schladen, Neuhäuser-Felde). Bei den Böden mit niedrigeren Reduktionsmittelgehalten (Bühren, Dinklage, Fuhrberg, Freistatt, Markhausen, Oldendorf und Vechtel) liegen die $\mathrm{R}_{\mathrm{NO} 3}$ nur zwischen 0,04 und $0,2 \mathrm{mg} \mathrm{kg}^{-1} \mathrm{~d}^{-1}$.

Die kumulierte Nitratreduktion von $\mathrm{T}_{0}$ bis 500 Tage nach Versuchsbeginn $\left(\mathrm{M}_{\mathrm{NO}} 500 \mathrm{~d}\right)$ wurde durch Summierung der reduzierten Mengen der unterschiedlichen Zeitintervalle berechnet (vgl. Abb. 5). $\mathrm{M}_{\mathrm{NO} 3} 500 \mathrm{~d}$ beträgt bei Böden mit hohem Reduktionsmittelvorrat 127 bis $479 \mathrm{mg} \mathrm{kg}^{-1}$ und bei Böden mit geringem Reduktionsmittelvorrat 10 bis $70 \mathrm{mg}$ $\mathrm{kg}^{-1}$.

Die kumulierte Nitratreduktion bezogen auf unendliche Inkubationszeit $\left(\mathrm{M}_{\mathrm{NO} 3}(\infty)\right)$ wurde durch Extrapolation ermittelt, indem die Reduktionsraten nach Ende der Messungen aus dem Trend der Reduktionsraten während des letzten und vorletzten Intervalls abgeleitet wurden.

Der Vergleich zwischen $\mathrm{M}_{\mathrm{NO} 3} 500 \mathrm{~d}$ und $\mathrm{M}_{\mathrm{NO} 3}(\infty)$ zeigt folgendes: In den Auen-Böden mit hohen Vorräten an Reduktionsmitteln (Aher-Kämpe, Reinshof, Schladen) ist $\mathrm{M}_{\mathrm{NO} 3}(\infty)$ viel höher als $\mathrm{M}_{\mathrm{NO} 3} 500 \mathrm{~d}$, d. h. nach 500 Tagen ist hier noch mit weiterer Nitratreduktion in erheblichen Umfang zu rechnen. Dagegen findet in den übrigen Böden nach 500 Tagen nur noch wenig Nitratreduktion statt, weil die Reduktionsmittelvorräte nahezu erschöpft sind.

Zur Beschreibung der Erschöpfbarkeit des Denitrifikationspotentials wurde ein Nachhaltigkeitsindex $\left(\mathrm{NI}_{\mathrm{NO} 3}\right)$ nach folgender Formel errechnet:

Nachhaltigkeitsindex $=\frac{\text { Nitratreduktionsrate nach } 500 \text { Tagen }}{\text { Nitratreduktionsrate zu Inkubationsbeginn }}$

$\mathrm{NI}_{\mathrm{NO} 3} 500 \mathrm{~d}$ variiert in den meisten Böden zwischen 0,31 und 0,56; d. h.; dass nach 500 Tagen noch 31 - 56 \% der Anfangsaktivität vorhanden war. Nur 4 Böden (Bühren, Dinklage, Neuhäuser Felde und Fuhrberg) zeigen mit $5-21 \%$ geringere Werte. $\mathrm{NI}_{\mathrm{NO} 3} 500 \mathrm{~d}$ scheint somit relativ unabhängig von Substrattyp und vom Reduktionsmittelvorrat zu sein. 
Tab. 8: Zusammenfassung der Ergebnisse zur Reduktion von Nitrat und Produktion von Sulfat aus den anaeroben und aeroben Inkubations-

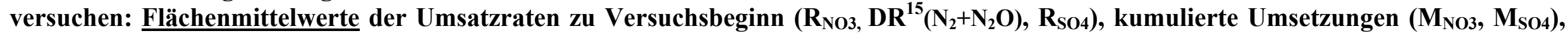
Anteil der heterotrophen Denitrifikation am Gesamtumsatz $\left(\mathrm{M}_{\mathrm{NO} 3} \mathrm{H}: \mathrm{M}_{\mathrm{NO} \text { (ges) }}\right)$, Nach haltigkeits-und Verfügbarkeits-Indizies (NI, VI)

\begin{tabular}{|c|c|c|c|c|c|c|c|c|c|c|c|}
\hline Messparameter & \multirow{2}{*}{$\begin{array}{l}\text { Aher- } \\
\text { Kämpe } \\
13\end{array}$} & \multirow{2}{*}{$\begin{array}{l}\text { Bühren } \\
18\end{array}$} & \multirow{2}{*}{$\begin{array}{l}\text { Reinshof } \\
20\end{array}$} & \multirow{2}{*}{$\begin{array}{l}\text { Schladen } \\
22\end{array}$} & \multicolumn{2}{|c|}{$\begin{array}{l}\text { Neuhäuser- Dinklage } \\
\text { Felde }\end{array}$} & Freistatt & Fuhrberg & $\begin{array}{l}\text { Mark- } \\
\text { hausen }\end{array}$ & Oldendorf & Vechtel \\
\hline $\mathrm{N}$ & & & & & 37 & 31 & 21 & 14 & 23 & 20 & 14 \\
\hline $\mathrm{R}_{\mathrm{NO} 3}$ & $1,56 \pm 0,81$ & $0,11 \pm 0,27$ & $1,12 \pm 0,84$ & $1,5 \pm 1,48$ & $1,01 \pm 1,35$ & $0,16 \pm 0,14$ & $0,03 \pm 0,04$ & $0,04 \pm 0,06$ & $0,14 \pm 0,14$ & $0,17 \pm 0,12$ & $0,12 \pm 0,11$ \\
\hline $\mathrm{DR}^{15}\left(\mathrm{~N}_{2}+\mathrm{N}_{2} \mathrm{O}\right)$ & $1,34 \pm 1,13$ & $0,17 \pm 0,29$ & n. b. & $1,05 \pm 0,74$ & $1,06 \pm 1,78$ & $0,19 \pm 0,28$ & $0,15 \pm 0,15$ & $0,11 \pm 0,07$ & $0,13 \pm 0,11$ & $0,06 \pm 0,11$ & $0 \pm 0$ \\
\hline $\mathrm{M}_{\mathrm{NO} 3} 500 \mathrm{~d}$ & $414 \pm 172$ & $9,7 \pm 44,4$ & $479 \pm 241$ & $343 \pm 291$ & $128 \pm 147$ & $46 \pm 42$ & $18 \pm 21$ & $11 \pm 13$ & $49 \pm 40$ & $71 \pm 40$ & $38 \pm 29$ \\
\hline $\mathrm{M}_{\mathrm{NO} 3}(\infty)$ & $1102 \pm 521$ & $13,3 \pm 18,2$ & $1027 \pm 452$ & $562 \pm 504$ & $134 \pm 145$ & $53 \pm 46$ & $30 \pm 111$ & $15 \pm 16$ & $72 \pm 56$ & $81 \pm 51$ & $39 \pm 28$ \\
\hline $\mathrm{NI}_{\mathrm{NO} 3} 500 \mathrm{~d}$ & $0,4 \pm 0,27$ & $0,05 \pm 0,21$ & $0,4 \pm 0,16$ & $0,36 \pm 0,21$ & $0,08 \pm 0,3$ & $0,17 \pm 0,31$ & $0,57 \pm 0,66$ & $0,13 \pm 0,3$ & $0,35 \pm 0,25$ & $0,56 \pm 0,39$ & $0,31 \pm 0,39$ \\
\hline $\mathrm{VI}_{\mathrm{NO} 3} 500 \mathrm{~d}$ & $0,03 \pm 0,02$ & $0,01 \pm 0,02$ & $0,05 \pm 0,04$ & $0,02 \pm 0,01$ & $0,03 \pm 0,03$ & $0,04 \pm 0,06$ & $0,02 \pm 0,01$ & $0,02 \pm 0,02$ & $0,03 \pm 0,04$ & $0,05 \pm 0,04$ & $0,04 \pm 0,03$ \\
\hline $\mathrm{VI}_{\mathrm{NO} 3}(\infty)$ & $0,07 \pm 0,06$ & $0,01 \pm 0,01$ & $0,11 \pm 0,08$ & $0,02 \pm 0,01$ & $0,03 \pm 0,02$ & $0,05 \pm 0,06$ & $0,02 \pm 0,01$ & $0,02 \pm 0,03$ & $0,04 \pm 0,06$ & $0,06 \pm 0,04$ & $0,04 \pm 0,03$ \\
\hline $\mathrm{M}_{\mathrm{NO} 3} 500 \mathrm{~d} \mathrm{H}$ & $354 \pm 175$ & $13,3 \pm 18,2$ & $369 \pm 132$ & $221 \pm 194$ & $91 \pm 88$ & $45 \pm 42$ & $18 \pm 21$ & $10,8 \pm 13,2$ & $44 \pm 37$ & $51 \pm 43$ & $38 \pm 29$ \\
\hline $\mathrm{M}_{\mathrm{NO} 3} \mathrm{H}: \mathrm{M}_{\mathrm{NO} 3 \mathrm{ges}} .500 \mathrm{~d}$ & $0,77 \pm 0,28$ & 1,00 & $0,82 \pm 0,17$ & $0,72 \pm 0,33$ & $0,68 \pm 0,35$ & $0,9 \pm 0,3$ & 1,00 & 1,00 & $0,85 \pm 0,28$ & $0,7 \pm 0,42$ & 1,00 \\
\hline $\mathrm{VI}_{\mathrm{NO} 3} 500 \mathrm{~d} \mathrm{H}$ & $0,02 \pm 0,01$ & $0,01 \pm 0,02$ & $0,04 \pm 0,04$ & $0,01 \pm 0,01$ & $0,02 \pm 0,02$ & $0,04 \pm 0,06$ & $0,02 \pm 0,01$ & $0,02 \pm 0,02$ & $0,03 \pm 0,04$ & $0,03 \pm 0,03$ & $0,04 \pm 0,03$ \\
\hline $\mathrm{R}_{\mathrm{SO} 4}$ & $0,84 \pm 0,7$ & $0 \pm 0$ & $1,14 \pm 1,20$ & $0,56 \pm 0,8$ & $0,44 \pm 0,6$ & $0,05 \pm 0,1$ & $0 \pm 0$ & $0 \pm 0$ & $0,07 \pm 0,10$ & $0,28 \pm 0,30$ & $0 \pm 0$ \\
\hline $\mathrm{M}_{\mathrm{SO} 4} 500 \mathrm{~d}$ & $88 \pm 78$ & $0 \pm 0$ & $164 \pm 197$ & $145 \pm 204$ & $59 \pm 101$ & $1,42 \pm 4,50$ & $0 \pm 0$ & $0 \pm 0$ & $5,2 \pm 8,8$ & $18 \pm 20$ & $0 \pm 0$ \\
\hline $\mathrm{M}_{\mathrm{SO} 4}(\infty)$ & $105 \pm 86$ & $0 \pm 0$ & $225 \pm 295$ & $214 \pm 305$ & $59 \pm 103$ & $2,18 \pm 5,0$ & $0 \pm 0$ & $0 \pm 0$ & $6,3 \pm 8,6$ & $21 \pm 21$ & $0 \pm 0$ \\
\hline $\mathrm{NI}_{\mathrm{SO} 4} 500 \mathrm{~d}$ & $0,36 \pm 0,27$ & $0 \pm 0$ & $0,1 \pm 0,13$ & $0,43 \pm 0,44$ & $0,16 \pm 0,18$ & $0,02 \pm 0,1$ & $0 \pm 0$ & $0 \pm 0$ & $0,27 \pm 0,28$ & $0,16 \pm 0,25$ & $0 \pm 0$ \\
\hline $\mathrm{VI}_{\mathrm{SO} 4} 500 \mathrm{~d}$ & $0,21 \pm 0,27$ & $0 \pm 0$ & $0,21 \pm 0,3$ & $0,33 \pm 0,23$ & $0,06 \pm 0,11$ & $0,03 \pm 0,09$ & $0 \pm 0$ & $0 \pm 0$ & $0,06 \pm 0,08$ & $0,12 \pm 0,12$ & $0 \pm 0$ \\
\hline $\mathrm{VI}_{\mathrm{SO} 4}(\infty)$ & $0,29 \pm 0,3$ & $0 \pm 0$ & $0,28 \pm 0,32$ & $0,51 \pm 0,32$ & $0,12 \pm 0,15$ & $0,09 \pm 0,24$ & $0 \pm 0$ & $0 \pm 0$ & $0,06 \pm 0,08$ & $0,15 \pm 0,13$ & $0 \pm 0$ \\
\hline $\mathrm{M}_{\mathrm{SO} 4} 500 \mathrm{~d} \mathrm{~A}$ & $281 \pm 235$ & $0 \pm 0$ & n. b. & $222 \pm 297$ & $162 \pm 216$ & $7,54 \pm 14,3$ & $0 \pm 0$ & $0 \pm 0$ & $54 \pm 49$ & $65 \pm 55$ & $0 \pm 0$ \\
\hline $\mathrm{M}_{\mathrm{SO} 4}(\infty) \mathrm{A}$ & $293 \pm 223$ & $0 \pm 0$ & n. b. & $274 \pm 287$ & $163 \pm 219$ & $7,84 \pm 12,8$ & $0 \pm 0$ & $0 \pm 0$ & $67 \pm 56$ & $70 \pm 57$ & $0 \pm 0$ \\
\hline $\mathrm{NI}_{\mathrm{SO} 4} 500 \mathrm{~d} \mathrm{~A}$ & $0,09 \pm 0,08$ & $0 \pm 0$ & n. b. & $0,02 \pm 0,04$ & $0,07 \pm 0,11$ & $0,26 \pm 0,16$ & $0 \pm 0$ & $0 \pm 0$ & $0,03 \pm 0,02$ & $0,15 \pm 0,15$ & $0 \pm 0$ \\
\hline $\mathrm{VI}_{\mathrm{SO} 4} 500 \mathrm{~d} \mathrm{~A}$ & $0,51 \pm 0,31$ & $0 \pm 0$ & n. b. & $0,43 \pm 0,51$ & $0,16 \pm 0,19$ & $0,19 \pm 0,39$ & $0 \pm 0$ & $0 \pm 0$ & $0,9 \pm 0,9$ & $0,57 \pm 0,4$ & $0 \pm 0$ \\
\hline $\begin{array}{ll}\mathrm{N} & =\text { Anze } \\
\mathrm{R} & =\text { Deni } \\
\mathrm{DR}^{15}(\mathrm{~N} 2+\mathrm{N} 2 \mathrm{O}) & ={ }^{15}\left(\mathrm{~N}_{2}\right. \\
\mathrm{M} & =\text { Kum } \\
\mathrm{NO}_{3} & =\text { Nitra } \\
\mathrm{SO}_{4} & =\text { Sulfa } \\
500 \mathrm{~d} & =\text { Bezc } \\
\mathrm{M}_{\mathrm{NO}} \mathrm{H}_{\mathrm{H}} \mathrm{M}_{\mathrm{NO} \text { gges. }} & =\text { Verh }\end{array}$ & $\begin{array}{l}\text { רl der Prober } \\
\text { ifikationsrate } \\
-\mathrm{N}_{2} \mathrm{O} \text { )-Denitr } \\
\text { lierte Menge } \\
\text { reduktion } \\
\text { produktion } \\
\text { gen auf die I } \\
\text { ltnis heterot }\end{array}$ & $\begin{array}{l}\text { zu Versuch } \\
\text { ikationsrate } \\
\text { in } \mathrm{mg} \mathrm{N} \mathrm{kg}\end{array}$ & $\begin{array}{l}\text { beginn in } \mathrm{m} \\
\text { in } \mathrm{mg} \mathrm{N} \mathrm{kg}^{-1} \\
\text { bzw.mg S k }\end{array}$ & $g^{-1} d^{-1}$ & ation & & $\begin{array}{l}=A \\
=S \\
=D\end{array}$ & $\begin{array}{l}\text { zogen auf u } \\
\text { chhaltigkeit } \\
\text { rfügbarkeits } \\
\text { andardabe Sulfatp } \\
\text { fferenz } \\
\text { ht bestimmt }\end{array}$ & $\begin{array}{l}\text { nendliche I } \\
\text { sindex } \\
\text { index } \\
\text { roduktion } \\
\text { ichung }\end{array}$ & kubationszeit & \\
\hline
\end{tabular}


Um die Verfügbarkeit des Reduktionsmittelvorrats für die Denitrifikation zu klassifizieren, wurde ein Verfügbarkeitsindex der Nitratreduktion $\left(\mathrm{VI}_{\mathrm{NO} 3}\right)$ nach folgender Formel ermittelt:

$\mathrm{VI}_{\mathrm{NO} 3} 500 \mathrm{~d}=\frac{\text { kumulierte Nitratreduktion nach } 500 \text { Tagen }}{\text { Reduktionsmittelvorrat }\left(\mathrm{C}_{\text {org }}+\text { Sulfid-S }\right)}$

$\mathrm{VI}_{\mathrm{NO} 3} 500 d$ liegt im Flächenmittel zwischen 0,01 und 0,05; d. h. 1 bis $5 \%$ des Reduktionsmittelvorrats war für die Denitrifikation während der ersten 500 Tagen verfügbar. $\mathrm{VI}_{\mathrm{NO} 3}(\infty)$ beträgt zwischen 0,01 und 0,11 (bei den meisten Böden 0,02 bis 0,07 ). Wie bei $\mathrm{NI}_{\mathrm{NO} 3}$ lässt sich bei $\mathrm{VI}_{\mathrm{NO} 3}$ kein eindeutiger Bezug zu Substrattyp oder Reduktionsmittelvorrat ablesen. Es ist bemerkenswert, dass die Spanne von $\mathrm{VI}_{\mathrm{NO}_{3}} 500 \mathrm{~d}(0,01-0,05)$ im Verhältnis zur Spanne von $\mathrm{R}_{\mathrm{NO} 3}(0,03-1,56)$ und $\mathrm{M}_{\mathrm{NO} 3}(10$ - 479) vergleichsweise klein ist. Das deutet darauf hin, dass allein aus dem Gesamt-Reduktionsmittelvorrat schon die Größenordnung der langfristigen Nitratreduktion abgeschätzt werden kann. In dem Vergleich zwischen $\mathrm{VI}_{\mathrm{NO}} 500 \mathrm{~d}$ und $\mathrm{VI}_{\mathrm{NO} 3}(\infty)$ zeigt sich wieder, dass bei Böden mit geringen Reduktionsmittelvorräten der Prozess nach 500 Tagen fast beendet ist, während bei Böden mit hohen Reduktionsmittelvorräten noch deutliche Nitratreduktion $\mathrm{zu}$ verzeichnen ist. Letzteres ist daran zu erkennen, dass $\mathrm{VI}_{\mathrm{NO} 3}(\infty)$ jeweils höher ist als $\mathrm{VI}_{\mathrm{NO} 3} 500 \mathrm{~d}$ (z.B. Aher Kämpe, Reinshof).

Der Anteil der heterotrophen Denitrifikation an der gesamten Nitratreduktion $\left(\mathrm{M}_{\mathrm{NO}} \mathrm{H}\right.$ : $\mathrm{M}_{\mathrm{NO3ges}}$.) wurde aus der Differenz zwischen Gesamtdenitrifikation und autotropher Denitrifikation ermittelt. Dabei wurde die autotrophe Denitrifikation aus der Sulfatproduktion abgeleitet. $\mathrm{M}_{\mathrm{NO} 3} \mathrm{H}: \mathrm{M}_{\mathrm{NO} \text { ges. }}$ beträgt für die sulfidhaltigen Böden zwischen 0,68 und 0,85. Somit ist der größte Teil der Nitratreduktion der heterotrophen Denitrifikation zuzuschreiben. In den sulfidfreien Böden findet ausschließlich heterotrophe Denitrifikation statt $\left(\mathrm{M}_{\mathrm{NO} 3} \mathrm{H}: \mathrm{M}_{\mathrm{NO} 3 \text { ges. }}=1\right)$. Unter den sulfidhaltigen Standorten wiesen Dinklage, Markhausen und Reinshof den höchsten Anteil an heterotropher Denitrifikation auf $(88 \%, 85 \%, 82 \%)$, während Neuhäuser-Felde den geringsten Anteil (68 \%) aufweist.

$\mathrm{R}_{\mathrm{SO} 4}$ ist die Sulfatproduktionsrate des ersten Inkubationsintervalls. Diese liegt bei den sulfidhaltigen Böden zwischen $0,05 \mathrm{mg} \mathrm{kg}^{-1} \mathrm{~d}^{-1}$ und $1,14 \mathrm{mg} \mathrm{kg}^{-1} \mathrm{~d}^{-1}$. R $\mathrm{SO} 4$ zeigt bei allen Flächen mit Ausnahme von Reinshof im Vergleich zu $\mathrm{R}_{\mathrm{NO} 3}$ geringere Werte. 
Die kumulierte Sulfatproduktion von $\mathrm{T}_{0}$ bis $\mathrm{zu} 500$ Tagen nach Versuchsbeginn ( $\left.\mathrm{M}_{\mathrm{SO} 4} 500 \mathrm{~d}\right)$ war mit Werten von 164, 145 und $59 \mathrm{mg} \mathrm{kg}^{-1}$ bei den sulfidreichen Standorten Reinshof und Schladen sowie Neuhäuser Felde am höchsten. Die kumulierte Sulfatproduktion bezogen auf unendliche Inkubationszeit $\left(\mathrm{M}_{\mathrm{SO} 4}(\infty)\right)$ ist nur unwesentlich höher als $\mathrm{M}_{\mathrm{SO} 4} 500 \mathrm{~d}$. Dies zeigt, dass bis 500 Tage nach Versuchsbeginn die Sulfidvorräte nahezu erschöpft sind. Dieser Befund lässt sich auch aus den Zeitkurven der Sulfatproduktion (vgl. Abb. 5) ablesen.

Die Nachhaltigkeits- und Verfügbarkeitsindizes für die Sulfatproduktion wurden im Prinzip wie für die Nitratreduktion errechnet (s.o.). Der Nachhaltigkeitsindex der Sulfatproduktion $\left(\mathrm{NI}_{\mathrm{SO} 4} 500 \mathrm{~d}\right)$ zeigt, dass nach 500 Tagen in den meisten Böden nur noch 2 - $43 \%$ der Anfangsaktivität vorhanden war. Der Standort Dinklage zeigt mit einem Wert von $3 \%$, dass der Sulfidvorrat praktisch erschöpft war.

Die Verfügbarkeit der Sulfatproduktion während der ersten 500 Tage (VI $\left.{ }_{\text {SO4 }} 500 d\right)$ beträgt für die einzelnen Flächenmittelwerte zwischen 3 und $33 \%$ des Sulfidvorrats. Für die Verfügbarkeit der kumulierten Sulfatproduktion bezogen auf unendliche Inkubationszeit $\left(\mathrm{VI}_{\mathrm{SO} 4}(\infty)\right)$ ergab sich Werte von 6 bis $51 \%$.

Ein Vergleich zwischen $\mathrm{VI}_{\mathrm{SO}_{4}} 500 \mathrm{~d}$ und $\mathrm{VI}_{\mathrm{SO} 4}(\infty)$ verdeutlicht nochmals, dass der größte Teil des verfügbaren Sulfidvorrats bis 500 Tage nach Versuchsbeginn aufoxidiert wur-

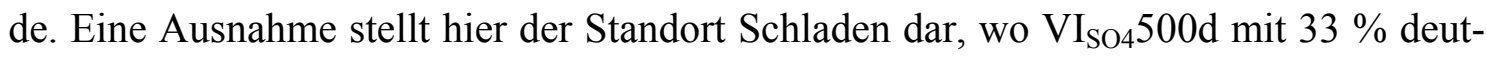
lich geringer ist als $\mathrm{VI}_{\mathrm{SO} 4}(\infty)$ mit $51 \%$. Im Gegensatz zu $\mathrm{VI}_{\mathrm{NO} 3}$ zeigt $\mathrm{VI}_{\mathrm{SO} 4}$ einen eindeutigen Bezug zu dem Reduktionsmittelvorrat und Substrattyp. $\mathrm{VI}_{\mathrm{SO} 4}$ ist bei den sulfidreichen Auen-Gleyen mit Werten zwischen 0,21 und 0,33 am höchsten.

$\mathrm{M}_{\mathrm{SO} 4} 500 \mathrm{dA}$ ist die kumulierte aerobe Sulfatproduktion, die durch Bebrütung in PEBeuteln bestimmt wurde. $\mathrm{M}_{\mathrm{SO} 4} 500 \mathrm{dA}$ liegt in allen sulfidhaltigen Böden mehrfach höher als die kumulierte anaerobe Sulfatproduktion $\left(\mathrm{M}_{\mathrm{SO} 4} 500 \mathrm{~d}\right)$. Folglich ist der Verfügbarkeitsindex für aerobe Bedingungen $\left(\mathrm{VI}_{\mathrm{SO}_{4}} 500 \mathrm{dA}\right)$ höher als für anaerobe Bedingungen $\left(\mathrm{VI}_{\mathrm{SO} 4} 500 \mathrm{~d}\right)$.

Der Nachhaltigkeitsindex der aeroben Sulfatproduktion ( $\left.\mathrm{NI}_{\mathrm{SO} 4} 500 \mathrm{dA}\right)$ beträgt zwischen 0,02 und 0,15 und ist somit geringer als der Nachhaltigkeitsindex der anaeroben Sulfatproduktion $\left(\mathrm{NI}_{\mathrm{SO} 4} 500 \mathrm{~d} ; 0,19-0,51\right)$.

Die Ergebnisse lassen sich wie folgt zusammenfassen:

- Die Böden mit hohen Reduktionsmittelvorräten weisen hohe initiale Abbauraten auf. 
- Bei den Böden mit geringen Reduktionsmittelvorräten kam der Nitratabbau bis 500 Tage nach Versuchsbeginn fast vollständig zum Erliegen.

- Die Verfügbarkeit der Reduktionsmittel für die Nitratreduktion bis 500 Tage nach Versuchsbeginn betrug $1-5 \%$ des Gesamtvorrats an $\mathrm{C}_{\text {org }}$ und Sulfid.

- 65 bis $100 \%$ der Gesamtdenitrifikation war auf heterotrophe Denitrifikation zurückzuführen.

- Die mittlere Rate der Sulfatporduktion liegt in fast allen Fällen niedriger als die mittlere Rate der Nitratreduktion.

- In den sulfidhaltigen Böden wurden nennenswerte Sulfatmengen produziert, jedoch kam die Sulfatproduktion bei den meisten Proben bis 500 Tage nach $\mathrm{T}_{0}$ vollständig zum Erliegen.

- 3 bis $33 \%$ des Sulfidvorrats waren bis 500 Tage nach Versuchsbeginn für die Denitrifikation verfügbar.

- Die kumulierte aerobe Sulfatproduktion von $\mathrm{T}_{0}$ bis 500 Tage nach Versuchsbeginn liegt bei allen Proben im Vergleich zu der anaeroben Sulfatproduktion höher.

\section{Statistische Auswertung}

\subsection{Test auf Normalverteilung}

Sämtliche Messgrößen wurden unter Verwendung des KOLMOGOROV-SMIRNOV-Tests auf Normalverteilung geprüft. Dabei zeigte sich, dass nur bei wenigen Messgrößen (pHWert und $\mathrm{C}_{\mathrm{hwl}}$ ) eine Normalverteilung abgesichert werden konnte. Zusätzlich wurden alle Daten log- transformiert, und dann nochmals auf Normalverteilung geprüft. Bei den nachfolgend aufgezählten Variablen kamen die nicht-transformierten Daten einer Normalverteilung näher als die log-transformierten Daten.

Abhängige Variablen: kumulierte Nitratreduktion bis 500 Tage nach $\mathrm{T}_{0}\left(\mathrm{M}_{\mathrm{NO} 3} 500 \mathrm{~d}\right)$, kumulierte heterotrophe Denitrifikation bis 500 Tage nach $\mathrm{T}_{0}\left(\mathrm{M}_{\mathrm{NO} 3} 500 \mathrm{dH}\right)$, kumulierte Sulfatproduktion bis 500 Tage nach $\mathrm{T}_{0}\left(\mathrm{M}_{\mathrm{SO} 4} 500 \mathrm{~d}\right)$, kumulierte Sulfatproduktion bezogen auf unendliche Inkubationszeit $\left(\mathrm{M}_{\mathrm{SO} 4}(\infty)\right)$, Verfügbarkeitsindex der Sulfatproduktion bis zu 500 Tagen nach $\mathrm{T}_{0}\left(\mathrm{VI}_{\mathrm{SO}_{4}} 500 \mathrm{~d}\right)$, Verfügbarkeitsindex der Sulfatproduktion bezogen auf unendliche Inkubationszeit $\left(\mathrm{VI}_{\mathrm{SO} 4}(\infty)\right)$, Nachhaltigkeitsindex der aeroben 
Sulfatproduktion bis 500 Tage nach $\mathrm{T}_{0}\left(\mathrm{NI}_{\mathrm{SO} 4} 500 \mathrm{dA}\right)$, Verfügbarkeitsindex der aeroben Sulfatproduktion bis 500 Tage nach $\mathrm{T}_{0}\left(\mathrm{VI}_{\mathrm{SO} 4} 500 \mathrm{dA}\right)$.

Unabhängigen Variablen: $\mathrm{C} / \mathrm{N}, \mathrm{CaCO}_{3}, \mathrm{pH}, \mathrm{C}_{\mathrm{lös}}, \mathrm{C}_{\mathrm{hwl}}$, Sandgehalt.

Bei allen anderen Variablen zeigten die log-transformierten Daten eine bessere, wenngleich nicht signifikante Anpassung an die Normalverteilung. Bei den aufgezählten Variablen wurden in der nachfolgenden multiplen Regression immer die nicht transformierten Daten, bei den übrigen Variablen die log-transformierten Daten verwendet. Wegen der mangelhaften Übereinstimmung der meisten Variablen mit der Normalverteilung wurde für die Korrelationsanalyse der SPEARMAN-Rangtest verwendet.

\subsection{Korrelationsanalyse}

Folgende Variablen wurden mit Hilfe der SPEARMAN-Rangtests miteinander verglichen. Abhängige Variablen für die Nitratreduktion: Nitratreduktionsrate zu Versuchsbeginn $\left(\mathrm{R}_{\mathrm{NO} 3}\right)$, kumulierte Nitratreduktion bis zu 500 Tagen nach $\mathrm{T}_{0}\left(\mathrm{M}_{\mathrm{NO} 3} 500 \mathrm{~d}\right)$, kumulierte Nitratreduktion bezogen auf unendliche Inkubationszeit $\left(\mathrm{M}_{\mathrm{NO} 3}(\infty)\right)$, Nachhaltigkeitsindex der Nitratreduktion bis 500 Tagenach $\mathrm{T}_{0} \quad\left(\mathrm{NI}_{\mathrm{NO} 3} 500 \mathrm{~d}\right)$, Nachhaltigkeitsindex bezogen auf unendliche Inkubationszeit $\left(\mathrm{NI}_{\mathrm{NO} 3}(\infty)\right)$, Verfügbarkeitsindex der Nitratreduktion bis 500 Tage nach $\mathrm{T}_{0}\left(\mathrm{VI}_{\mathrm{NO} 3} 500 \mathrm{~d}\right)$, Verfügbarkeitsindex der Nitratreduktion bezogen auf die unendliche Inkubationszeit $\left(\mathrm{VI}_{\mathrm{NO} 3}(\infty)\right)$, die kumulierte heterotrophe Denitrifikation bis 500 Tage nach $\mathrm{T}_{0}$, $\left(\mathrm{M}_{\mathrm{NO} 3} 500 \mathrm{dH}\right)$ und der Verfügbarkeitsindex der heterotrophen Denitrifikation bis zu 500 Tagen nach $\mathrm{T}_{0}\left(\mathrm{VI}_{\mathrm{NO} 3} 500 \mathrm{dH}\right)$.

Abhängige Variablen für die anaerobe Sulfatproduktion: kumulierte Sulfatproduktion bis 500 Tage nach $\mathrm{T}_{0}\left(\mathrm{M}_{\mathrm{SO} 4} 500 \mathrm{~d}\right)$, kumulierte Sulfatproduktion bezogen auf die unendliche Inkubationszeit $\left(\mathrm{M}_{\mathrm{SO} 4}(\infty)\right)$, Nachhaltigkeitsindex der Sulfatproduktion bis zu 500 Tagen nach $\mathrm{T}_{0}\left(\mathrm{NI}_{\mathrm{SO} 4} 500 \mathrm{~d}\right)$, Verfügbarkeitsindex der Sulfatproduktion bis zu 500 Tagen nach $\mathrm{T}_{0}\left(\mathrm{VI}_{\mathrm{SO} 4} 500 \mathrm{~d}\right)$, Verfügbarkeitsindex der Sulfatproduktion bezogen auf die unendliche Inkubationszeit $\left(\mathrm{VI}_{\mathrm{SO} 4}(\infty)\right)$.

Aerobe Sulfatproduktion: kumulierte Sulfatproduktion bis 500 Tage nach $\mathrm{T}_{0}$ $\left(\mathrm{M}_{\mathrm{SO} 4} 500 \mathrm{dA}\right)$, Nachhaltigkeitsindex der Sulfatproduktion bis 500 Tage nach $\mathrm{T}_{0}$ $\left(\mathrm{NI}_{\mathrm{SO} 4} 500 \mathrm{dA}\right)$, Verfügbarkeitsindex der Sulfatproduktion bis 500 Tage nach $\mathrm{T}_{0}$ $\left(\mathrm{VI}_{\mathrm{SO} 4} 500 \mathrm{dA}\right)$. 
Unabhängige Bodenparameter: $\mathrm{C}_{\mathrm{org}}, \mathrm{C}_{\mathrm{hwl}}, \mathrm{C}_{\mathrm{lös}}, \mathrm{C} / \mathrm{N}, \mathrm{CaCO}_{3}$, Sulfid, pH, $\mathrm{Fe}_{\mathrm{lös}}$ Sand-, Schluff- und Tongehalt.

Die Korrelationskoeffizienten sind in der Tab. 9 a, b zusammengefasst. Tab. 9a zeigt die Korrelationen innerhalb der Gruppe der unabhängigen Variablen.

Anhand der Korrelationen der Texturvariablen mit den übrigen unabhängigen Parametern lassen sich zwei Gruppen unterscheiden: 1. Carbonatfreie Sandböden mit hohen Werten an $\mathrm{C} / \mathrm{N}, \mathrm{Fe}_{\text {lös }}$ und geringen Werte an $\mathrm{pH}, \mathrm{C}_{\text {org }}, \mathrm{C}_{\mathrm{hwl}}, \mathrm{C}_{\text {lös }}$ und Sulfid. Der Sandgehalt zeigt signifikante negative Korrelationen mit $C_{o r g}(r=-0,45)$, Sulfid $(r=-0,42), C_{h w l}$ $(r=-0,20)$, und $\mathrm{CaCO}_{3}(r=-0,48)$ sowie mit dem $\mathrm{pH}-$ Wert $(r=-0,64)$. Dagegen besteht eine positive Beziehung zwischen dem Sandgehalt und $\mathrm{Fe}_{\text {lös }}(\mathrm{r}=0,52)$ sowie dem $\mathrm{C} / \mathrm{N}$ Verhältnis $(\mathrm{r}=0,74)$.

Zur zweiten Gruppe gehören die Lehmböden, die im Gegensatz zu den Sandböden durch hohe Werte an $\mathrm{C}_{\mathrm{org}}, \mathrm{C}_{\mathrm{hwl}}$, Sulfid, $\mathrm{CaCO}_{3}, \mathrm{pH}, \mathrm{C} / \mathrm{N}-$ Verhältnis und $\mathrm{Fe}_{\text {lös }}$ gekennzeichnet sind. Dabei sind die Parameter $\mathrm{C}_{\mathrm{org}}, \mathrm{C}_{\mathrm{hwl}}$, Sulfid, $\mathrm{CaCO}_{3}$ und $\mathrm{pH}$ positiv mit Schluff- und Ton-Gehalt korreliert.

Innerhalb der unabhängigen Variablen ist die Korrelation zwischen $\mathrm{C}_{\text {org }}$ und Sulfid am stärksten ausgeprägt. Es bestehen aber auch positive, hoch signifikante Korrelationen zwischen $\mathrm{C}_{\text {org }}, \mathrm{C}_{\mathrm{hwl}}$, Sulfid, $\mathrm{CaCO}_{3}$ und $\mathrm{pH}$. Dagegen wurden zwischen $\mathrm{C}_{\text {lös }}$ und den anderen unabhängigen Variablen nur relativ gering ausgeprägte Beziehungen beobachtet. Zwischen Sulfid und $\mathrm{C}_{\text {lös }}$ besteht keine Korrelation.

$\mathrm{C}_{\text {lös }}$-Gehalte und das $\mathrm{C} / \mathrm{N}-$ Verhältnis zeigen vergleichsweise geringe Beziehungen zu $\mathrm{C}_{\text {org }}, \mathrm{C}_{\mathrm{hwl}}$ und Sulfid.

Tab. 9 b zeigt die Korrelationen zwischen den abhängigen und den unabhängigen Variablen. Daraus ergeben sich höchst signifikante Beziehungen $(\mathrm{P}<0,001)$ zwischen $\mathrm{C}_{\text {org }}$ und folgenden Parametern: $\mathrm{R}_{\mathrm{NO} 3}(\mathrm{r}=0,60), \mathrm{M}_{\mathrm{NO} 3} 500 \mathrm{~d}(\mathrm{r}=0,73), \mathrm{M}_{\mathrm{NO} 3}(\infty)(\mathrm{r}=0,70)$, $\mathrm{M}_{\mathrm{SO} 4} 500 \mathrm{~d}(\mathrm{r}=0,58), \mathrm{M}_{\mathrm{SO} 4}(\infty)(\mathrm{r}=0,68), \mathrm{NI}_{\mathrm{SO} 4} 500 \mathrm{~d}(\mathrm{r}=0,58), \mathrm{VI}_{\mathrm{SO} 4} 500 \mathrm{~d}(\mathrm{r}=0,45)$, $\mathrm{VI}_{\mathrm{SO} 4}(\infty)(\mathrm{r}=0,59)$. Der Sulfidgehalt zeigt positive Beziehungen zu den gleichen Parametern, wobei die Korrelationskoeffizienten noch höher liegen.

Höchst signifikante Beziehungen existieren auch zwischen $\mathrm{C}_{\mathrm{hwl}}$ und einigen abhängigen Parametern $\left(\mathrm{R}_{\mathrm{NO} 3}, \mathrm{M}_{\mathrm{NO} 3} 500 \mathrm{~d}, \mathrm{M}_{\mathrm{NO} 3}(\infty), \mathrm{M}_{\mathrm{NO} 3} 500 \mathrm{dH}, \mathrm{M}_{\mathrm{SO} 4} 500 \mathrm{~d}, \mathrm{M}_{\mathrm{SO} 4}(\infty), \mathrm{NI}_{\mathrm{SO} 4} 500 \mathrm{~d}\right.$, $\left.\mathrm{VI}_{\mathrm{SO} 4} 500 \mathrm{~d}, \mathrm{VI}_{\mathrm{SO} 4}(\infty)\right)$. 
Tab. 9a: SPEARMAN-Rang Korrelationskoeffizienten der unabhängigen Bodenparameter $(\mathrm{N}=\mathbf{2 3 3})$

\begin{tabular}{|c|c|c|c|c|c|c|c|c|c|c|c|}
\hline & $\mathrm{C}_{\text {org }}$ & DOC & $\mathbf{C}_{\mathrm{hwl}}$ & $\mathrm{C} / \mathrm{N}$ & Sulfid & $\mathrm{CaCO}_{3}$ & $\mathrm{pH}$ & $\mathbf{F e}_{\text {lös }}$ & Sand & Schluff & Ton \\
\hline$\overline{C_{\text {org }}}$ & 1 & & & & & & & & & & \\
\hline DOC & $0,31^{* * *}$ & 1 & & & & & & & & & \\
\hline$C_{\text {hwl }}$ & $0,67 * * *$ & $0,17^{*}$ & 1 & & & & & & & & \\
\hline$C / N_{t}$ & $-0,09$ ns & $0,17^{* *}$ & $-0,10 \mathrm{~ns}$ & 1 & & & & & & & \\
\hline Sulfid & $0,72^{* * *}$ & $0,10 \mathrm{~ns}$ & $0,4^{* * *}$ & $-0,18^{* *}$ & 1 & & & & & & \\
\hline $\mathrm{CaCO}_{3}$ & $0,43^{* * *}$ & $0,23^{* * *}$ & $0,53^{* * *}$ & $-0,38^{* * *}$ & $0,54 * * *$ & 1 & & & & & \\
\hline pH & $0,37^{* * *}$ & $0,13^{*}$ & $0,31^{* * *}$ & $-0,57^{* * *}$ & $0,43^{* * *}$ & $0,68^{* * *}$ & 1 & & & & \\
\hline $\mathrm{Fe}_{\text {lös }}$ & $-0,36 * * *$ & $-0,18^{* *}$ & $-0,32^{* * *}$ & $0,48 * * *$ & $-0,23^{* * *}$ & $-0,42 * * *$ & $-0,60 * * *$ & 1 & & & \\
\hline Sand & $-0,45^{* * *}$ & $-0,03$ ns & $-0,20 * *$ & $0,74^{* * *}$ & $-0,42^{* * *}$ & $-0,48^{* * *}$ & $-0,64^{* * *}$ & $0,52^{* * *}$ & 1 & & \\
\hline Schluff & $0,43^{* * *}$ & $0,03 \mathrm{~ns}$ & $0,22^{* *}$ & $-0,72^{* * *}$ & $0,41 * * *$ & $0,52^{* * *}$ & $0,65^{* * *}$ & $-0,51 * * *$ & $-0,96 * * *$ & 1 & \\
\hline Ton & $0,50 * * *$ & $0,13^{*}$ & $0,30 * * *$ & $-0,68^{* * *}$ & $0,42 * * *$ & $0,39 * * *$ & $0,54 * * *$ & $-0,51^{* * *}$ & $-0,87^{* * *}$ & $0,78 * * *$ & 1 \\
\hline
\end{tabular}

n.s. $\quad=$ nicht signifikant

$*, * *, * * \quad=$ Signifikanzniveau $(\mathrm{P}<0,05 ; 0,01 ; 0,001)$ 
Tab. 9b: SPEARMAN-Rang Korrelationskoeffizienten der abhängigen Bodenparameter $(\mathrm{N}=\mathbf{2 3 3})$

\begin{tabular}{|c|c|c|c|c|c|c|c|c|c|c|c|}
\hline & $C_{\text {org }}$ & DOC & $\mathbf{C}_{\text {hwl }}$ & $\mathbf{C} / \mathbf{N}$ & Sulfid & $\mathrm{CaCO}_{3}$ & pH & $\mathrm{Fe}_{\text {Iös }}$ & Sand & Schluff & Ton \\
\hline $\mathbf{R}_{\mathrm{NO} 3}$ & $0,60^{* * *}$ & $0,14^{*}$ & $0,29 * * *$ & $-0,06 \mathrm{~ns}$ & $0,66^{* * *}$ & $0,46 * * *$ & $0,35^{* * *}$ & $-0,22 * * *$ & $-0,32 * * *$ & $0,32^{* * *}$ & $0,27^{* * *}$ \\
\hline $\mathrm{DR}^{15}(\mathrm{~N} 2+\mathrm{N} 2 \mathrm{O})$ & $0,54^{* * *}$ & $0,19 * *$ & $0,34 * * *$ & $-0,10 \mathrm{~ns}$ & $0,47^{* * *}$ & $0,38 * * *$ & $0,08 \mathrm{~ns}$ & $-0,10 \mathrm{~ns}$ & $-0,29 * * *$ & $0,28^{* * *}$ & $0,37^{* * *}$ \\
\hline $\mathbf{M}_{\mathrm{NO} 3} 500 \mathrm{~d}$ & $0,73^{* * *}$ & $0,13^{*}$ & $0,39 * * *$ & $-0,13^{*}$ & $0,73^{* * *}$ & $0,50 * * *$ & $0,42 * * *$ & $-0,24^{* * *}$ & $-0,38 * * *$ & $0,36^{* * *}$ & $0,35^{* * *}$ \\
\hline $\mathrm{M}_{\mathrm{NO} 3}(\infty)$ & $0,70 * * *$ & $0,16^{* *}$ & $0,38 * * *$ & $-0,05 \mathrm{~ns}$ & $0,70 * * *$ & $0,46 * * *$ & $0,35^{* * *}$ & $-0,21^{* * *}$ & $-0,29 * * *$ & $0,26^{* * *}$ & $0,30 * * *$ \\
\hline $\mathrm{NI}_{\mathrm{NO} 3} 500 \mathrm{~d}$ & $0,17^{* *}$ & $0,14^{*}$ & $0,17^{*}$ & $0,04 \mathrm{~ns}$ & $0,07 \mathrm{~ns}$ & $0,01 \mathrm{~ns}$ & $0,04 \mathrm{~ns}$ & $-0,06 \mathrm{~ns}$ & $0,02 \mathrm{~ns}$ & $-0,05 \mathrm{~ns}$ & $0,06 \mathrm{~ns}$ \\
\hline $\mathrm{V}_{\mathrm{NO} 3} 500 \mathrm{~d}$ & $-0,04 \mathrm{~ns}$ & $-0,12^{*}$ & $-0,06 \mathrm{~ns}$ & $-0,06 n s$ & $0,19 * *$ & $0,22 * * *$ & $0,17^{* *}$ & $0,06 \mathrm{~ns}$ & $-0,01 \mathrm{~ns}$ & $0,00 \mathrm{~ns}$ & $-0,09 \mathrm{~ns}$ \\
\hline $\mathbf{V} \mathbf{I}_{\mathrm{NO} 3}(\infty)$ & $0,06 \mathrm{~ns}$ & $-0,05 \mathrm{~ns}$ & $-0,04 \mathrm{~ns}$ & $-0,05 n s$ & $0,27^{* * *}$ & $0,28 * * *$ & $0,21 * * *$ & $0,02 \mathrm{~ns}$ & $-0,05 \mathrm{~ns}$ & $0,02 \mathrm{~ns}$ & $-0,01 \mathrm{~ns}$ \\
\hline $\mathrm{M}_{\mathrm{NO} 3} 500 \mathrm{dH}$ & $0,65^{* * *}$ & $0,14^{*}$ & $0,39 * * *$ & $-0,16^{* *}$ & $0,50 * * *$ & $0,44 * * *$ & $0,39 * * *$ & $-0,26 * * *$ & $-0,4^{* * *}$ & $0,39 * * *$ & $0,36^{* * *}$ \\
\hline $\mathrm{V}_{\mathrm{NO} 3} 500 \mathrm{dH}$ & $-0,12^{*}$ & $-0,12^{*}$ & $-0,07 \mathrm{~ns}$ & $-0,10 \mathrm{~ns}$ & $-0,13^{*}$ & $0,06 \mathrm{~ns}$ & $0,06 \mathrm{~ns}$ & $0,03 \mathrm{~ns}$ & $-0,03 \mathrm{~ns}$ & $0,02 \mathrm{~ns}$ & $-0,06 \mathrm{~ns}$ \\
\hline$M_{s 04} 500 d$ & $0,58^{* * *}$ & $0,00 \mathrm{~ns}$ & $0,33^{* * *}$ & $-0,21 * * *$ & $0,83^{* * *}$ & $0,52 * * *$ & $0,46^{* * *}$ & $-0,24^{* * *}$ & $-0,42 * * *$ & $0,42^{* * *}$ & $0,41^{* * *}$ \\
\hline $\mathrm{M}_{\mathrm{SO} 4}(\infty)$ & $0,68^{* * *}$ & $0,13^{*}$ & $0,43^{* * *}$ & $-0,12^{*}$ & $0,86^{* * *}$ & $0,53^{* * *}$ & $0,40 * * *$ & $-0,24^{* * *}$ & $-0,32 * * *$ & $0,33^{* * *}$ & $0,33^{* * *}$ \\
\hline $\mathrm{NI}_{\mathrm{SO} 4} 500 \mathrm{~d}$ & $0,58^{* * *}$ & $0,29 * * *$ & $0,31 * * *$ & $-0,19 * *$ & $0,58^{* * *}$ & $0,36 * * *$ & $0,37^{* * *}$ & $-0,26^{* * *}$ & $-0,26^{* * *}$ & $0,25^{* * *}$ & $0,38^{* * *}$ \\
\hline $\mathrm{V}_{\mathrm{SO} 4} 500 \mathrm{~d}$ & $0,45^{* * *}$ & $-0,05 \mathrm{~ns}$ & $0,20 * *$ & $-0,17^{* *}$ & $0,72^{* * *}$ & $0,30 * * *$ & $0,35^{* * *}$ & $-0,10 \mathrm{~ns}$ & $-0,34^{* * *}$ & $0,32^{* * *}$ & $0,34^{* * *}$ \\
\hline $\mathbf{V I}_{\mathrm{SO} 4}(\infty)$ & $0,59 * * *$ & $0,11^{*}$ & $0,33^{* * *}$ & $-0,14^{*}$ & $0,74^{* * *}$ & $0,37^{* * *}$ & $0,34^{* * *}$ & $-0,18^{*} *$ & $-0,30 * * *$ & $0,31 * * *$ & $0,31^{* * *}$ \\
\hline$M_{s 04} 500 d A$ & $0,41^{* * *}$ & $0,07 \mathrm{~ns}$ & $0,13 \mathrm{~ns}$ & $0,15^{*}$ & $0,67^{* * *}$ & $0,38 * * *$ & $0,08 \mathrm{~ns}$ & $0,03 \mathrm{~ns}$ & $-0,04 \mathrm{~ns}$ & $0,03 \mathrm{~ns}$ & $-0,02 \mathrm{~ns}$ \\
\hline$M_{S O 4}(\infty) A$ & $0,48^{* * *}$ & $0,11 \mathrm{~ns}$ & $0,19 *$ & $0,17^{* *}$ & $0,68^{* * *}$ & $0,40 * * *$ & $0,17^{* *}$ & $0,04 \mathrm{~ns}$ & $0,0 \mathrm{~ns}$ & $0,00 \mathrm{~ns}$ & $-0,03 \mathrm{~ns}$ \\
\hline $\mathrm{NI}_{\mathrm{sO} 4} 500 \mathrm{dA}$ & $0,12^{*}$ & $0,04 \mathrm{~ns}$ & $0,14^{*}$ & $0,41 * * *$ & $0,21^{* * *}$ & $0,05 \mathrm{~ns}$ & $-0,21^{* *}$ & $0,31 * * *$ & $0,27^{* * *}$ & $-0,26 * * *$ & $-0,29 * * *$ \\
\hline $\mathrm{VI}_{\mathrm{so} 4} 500 \mathrm{dA}$ & $0,12^{*}$ & $0,13^{*}$ & $-0,10 \mathrm{~ns}$ & $0,14^{*}$ & $0,40^{* * *}$ & $0,08 \mathrm{~ns}$ & $-0,07$ ns & $0,06 \mathrm{~ns}$ & $0,06 \mathrm{~ns}$ & $-0,09$ ns & $-0,06$ ns \\
\hline $\begin{array}{l}\mathrm{R} \\
\mathrm{DR}^{15}(\mathrm{~N} 2+\mathrm{N} 2 \mathrm{O}) \\
\mathrm{M} \\
500 \mathrm{~d} \\
(\infty) \\
\mathrm{NO}_{3} \\
\text { n.s. }\end{array}$ & \multicolumn{6}{|c|}{$\begin{array}{l}=\text { Denitrifikationsrat } \\
={ }^{15}\left(\mathrm{~N}_{2}+\mathrm{N}_{2} \mathrm{O}\right) \text { Deniti } \\
=\text { Kumulierte Menge } \\
=\text { Bezogen auf Inkul } \\
=\text { Bezogen auf une } \\
=\text { Nitratreduktion } \\
=\text { nicht signifikant }\end{array}$} & $\begin{array}{l}\mathrm{SO}_{4} \\
\mathrm{H} \\
\mathrm{NI} \\
\mathrm{VI} \\
\mathrm{A} \\
*{ }^{*}{ }^{*},\end{array}$ & \multicolumn{4}{|c|}{$\begin{array}{l}=\text { Sulfatproduktion } \\
=\text { Heterotrophe Denitrifikation } \\
=\text { Nachhaltigkeitsindex } \\
=\text { Verfügbarkeitsindex } \\
=\text { Aerobe Sulfatproduktion } \\
=\text { Signifikanzniveau }(P<0,05 ; 0,01 ; 0,001)\end{array}$} \\
\hline
\end{tabular}


Der Nachhaltigkeitsindex für die Nitratreduktion $\left(\mathrm{NI}_{\mathrm{NO} 3}\right)$ ist mit $\mathrm{C}_{\text {org }}, \mathrm{C}_{\text {lös }}$ und $\mathrm{C}_{\mathrm{hwl}}$ mit geringeren Koeffizienten korreliert. Hingegen wurde keine Beziehung zwischen $\mathrm{NI}_{\mathrm{NO} 3}$ und den übrigen unabhängigen Parametern festgestellt.

Hohe Korrelationen bestehen zwischen den Verfügbarkeitsindizes für die Nitratreduktion $\left(\mathrm{VI}_{\mathrm{NO} 3} 500 \mathrm{~d}\right),\left(\mathrm{VI}_{\mathrm{NO} 3}(\infty)\right)$ und Sulfid, $\mathrm{CaCO}_{3}$ und $\mathrm{pH}$. Mit $\mathrm{C}_{\text {org }}, \mathrm{C}_{\mathrm{hwl}}$ und $\mathrm{C}_{\text {lös }}$ sind diese Variablen dagegen nicht korreliert. Geringe negative Korrelation besteht zwischen dem Verfügbarkeitsindex für die heterotrophe Denitrifikation $\left(\mathrm{VI}_{\mathrm{NO} 3} 500 \mathrm{dH}\right)$ und $\mathrm{C}_{\mathrm{org}}$, $\mathrm{C}_{\mathrm{hwl}}, \mathrm{C}_{\text {lös. }} \mathrm{Zu}$ den übrigen unabhängigen Parametern besteht keine signifikanteBeziehung.

Eine positiv hoch signifikante Korrelation ergab sich zwischen dem Nachhaltigkeitsindex der Sulfatproduktion $\left(\mathrm{NI}_{\mathrm{SO} 4} 500 \mathrm{~d}\right)$ und allen unabhängigen Variablen mit Ausnahme von $\mathrm{C} / \mathrm{N}, \mathrm{Fe}_{\text {lös }}$ und Sandgehalt.

Die Verfügbarkeitsindizes für die Sulfatproduktion $\left(\mathrm{VI}_{\mathrm{SO} 4} 500 \mathrm{~d}, \mathrm{VI}_{\mathrm{SO} 4}(\infty)\right)$ wiesen die stärksten Korrelationen mit $\mathrm{C}_{\text {org }}, \mathrm{C}_{\mathrm{hwl}}$, Sulfid, $\mathrm{CaCO}_{3}$ und $\mathrm{pH}$ auf.

$\mathrm{NI}_{\mathrm{SO} 4} 500 \mathrm{dA}$ ist mit den Parametern $\mathrm{C} / \mathrm{N}-$ Verhältnis, Sulfid, $\mathrm{pH}, \mathrm{Fe}_{\text {lös, }}$ Textur signifikant und mit Sulfid hoch signifikant korreliert. Eine geringe bzw. keine Beziehung besteht zwischen $\mathrm{VI}_{\mathrm{SO} 4} 500 \mathrm{~d}$ A und allen übrigen Parametern.

Die hohen Korrelationskoeffizienten zwischen den Gehalten an Reduktionsmitteln $\left(\mathrm{C}_{\text {org }}\right.$ und Sulfid) und den Variablen der aktuellen und langfristigen Denitrifikation $\left(\mathrm{R}_{\mathrm{NO} 3}\right.$, $\mathrm{M}_{\mathrm{NO} 3}$ ) deuten an, dass für eine Prognose der Denitrifikation den Gehalten an $\mathrm{C}_{\text {org }}$ und Sulfid eine Schlüsselrolle zukommt. Die mittels ${ }^{15} \mathrm{~N}$-Tracer-Technik ermittelten Denitrifikationsraten $\left({ }^{15}\left(\mathrm{~N}_{2}+\mathrm{N}_{2} \mathrm{O}\right)\right.$-Bildung) zeigen erwartungsgemäß genau wie $\mathrm{R}_{\mathrm{NO}}$ positive Beziehungen zu den Reduktionsmittelvorräten $\left(\mathrm{C}_{\mathrm{org}}: \mathrm{r}=0,54 ; \mathrm{C}_{\mathrm{hwl}}: \mathrm{r}=0,34\right.$; Sulfid: $\mathrm{r}=$ 0,47), wobei die Koeffizienten jedoch etwas geringer sind.

Die kumulierte Sulfatproduktion weist ebenfalls eine enge Korrelation zu den beiden Reduktionsmittelgehalten (Sulfid und $\mathrm{C}_{\text {org }}$ ) auf.

Die Nachhaltigkeits- und Verfügbarkeitsindizes für die Sulfatproduktion zeigen im Vergleich zu den Nachhaltigkeits- und den Verfügbarkeitsindizes für die Nitratreduktion relativ stärkere Beziehungen zu den unabhängigen Variablen.

Im Gegensatz zur Nitratreduktion $\left(\mathrm{R}_{\mathrm{NO} 3}, \mathrm{M}_{\mathrm{NO} 3} 500 \mathrm{~d}\right)$ zeigt die Verfügbarkeit des Reduktionsmittelvorrats $\left(\mathrm{VI}_{\mathrm{NO} 3} 500 \mathrm{~d}, \mathrm{VI}_{\mathrm{NO} 3}(\infty)\right)$ keine signifikante Beziehung zu $\mathrm{C}_{\text {org. }}$. Zwischen Felös und den meisten abhängigen Parametern besteht mit Ausnahme von $\mathrm{NI}_{\mathrm{SO} 4} 500 \mathrm{dA}$ eine negative bzw. keine Korrelation. Zusammenfassend ist folgendes festzuhalten: 
- Die Beziehung zwischen Texturparametern und Reduktionsmittelvorräten macht deutlich, dass die Sandböden im Gegensatz zu den Lehmböden durch vergleichsweise geringe $\mathrm{C}_{\text {org }}$ und Sulfid-Gehalte gekennzeichnet sind.

- Die Nitratreduktionsrate zu Versuchsbeginn und die kumulierte Nitratreduktion haben übereinstimmend eine signifikante Beziehung zu den meisten unabhängigen Parametern.

- Beide Messgrößen sind besonders gut mit $\mathrm{C}_{\text {org }}$ und Sulfid korreliert. Sie werden offenbar durch die gleichen Parameter gesteuert. Es ist zu erwarten, dass diese Variablen sich gut eignen, um die Nitratreduktion abzuschätzen.

- Außer den wesentlichen Einflussfaktoren $\left(\mathrm{C}_{\text {org }}\right.$ und Sulfid $)$ haben die übrigen unabhängigen Bodenparameter für die Denitrifikation eine untergeordnete Bedeutung.

- Die Korrelation zwischen den Nachhaltigkeits- und Verfügbarkeitsindizes der Nitratreduktion und den meisten unabhängigen Variablen ist nur schwach ausgeprägt. Es ist daher nicht zu erwarten, dass sich diese Größen anhand der unabhängigen Variablen vorhersagen lassen.

- Alle Variablen der Sulfatproduktion zeigen eine enge Beziehung zum Reduktionsmittelvorrat.

\subsection{Regressionsanalyse}

Durch die multiple Regressionsanalyse soll untersucht werden, inwieweit sich die abhängigen Parameter durch die unabhängigen Variablen mit einem empirischen Modell vorhersagen lassen.

Diese Auswertung erfolgte am gesamten Datensatz und zusätzlich an verschiedenen Teildatensätzen. Die Aufteilung des Datensatzes hat folgende Gründe:

1. Einige Parameter (z.B. $\mathrm{C}_{\mathrm{hwl}}, \mathrm{C}_{\text {lös }}$ und $\mathrm{Fe}_{\text {lös }}$ ) wurden nur an einem Teil der Proben gemessen.

2. Durch eine Aufteilung der Böden in Untergruppen, welche im Vergleich zur Gsamtgruppe in sich homogener sind, sollte die Anpassung der Regressionsmodelle an die Messdaten verbessert werden. Daher wurde der Datensatz in zwei Gruppen, nämlich in "Lehmböden" und in "Sandböden" aufgeteilt. Durch die Aufteilung ergeben sich die folgenden Datensätze: 1. Alle Proben; 2. Proben, für die Messwerte von $\mathrm{C}_{\mathrm{hwl}}, \mathrm{C}_{\text {lös }}$ und $\mathrm{Fe}_{\text {lös }}$ vorliegen; 3. Sandböden; 4. Lehmböden; 5. Sulfidhaltige Proben (in Tab. 10 mit „*“ gekennzeichnet). 
In Tab. A 5 (im Anhang) sind die errechneten Ergebnisse der Regressionsanalyse wiedergegeben. Dargestellt sind die Koeffizienten für die allgemeine lineare Gleichung $(\mathrm{y}=$ $\mathrm{a}+\mathrm{bx}_{1}+\mathrm{cx}_{2}+\ldots+\mathrm{zx}_{\mathrm{n}}$.). Die Regressionsanalyse erfolgte für folgende Auswahl der abhängigen Parameter (Bedeutung der verwendeten zusätzlichen Indizes: (Alle)= Datensatz ,alle Proben“, (L)= Datensatz „Lehmböden“, (S) = Datensatz „Sandböden“; * =

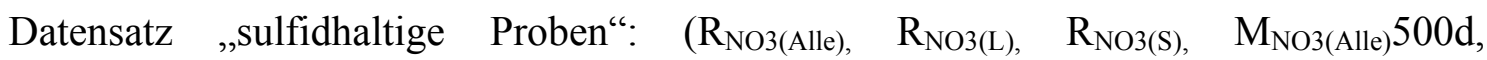
$\mathrm{M}_{\mathrm{NO} 3(\mathrm{All})}(\infty), \mathrm{M}_{\mathrm{NO} 3(\mathrm{~L})} 500 \mathrm{~d}, \mathrm{M}_{\mathrm{NO} 3(\mathrm{~L})}(\infty),\left(\mathrm{M}_{\mathrm{NO} 3(\mathrm{~S})} 500 \mathrm{~d}, \mathrm{M}_{\mathrm{NO} 3(\mathrm{~S})}(\infty), \mathrm{M}_{\mathrm{NO} 3(\mathrm{Alle})} \mathrm{H} 500 \mathrm{~d}\right.$, $\mathrm{M}_{\mathrm{NO} 3(\mathrm{All})} \mathrm{H}(\infty), \quad \mathrm{M}_{\mathrm{NO} 3(\mathrm{~L})} \mathrm{H} 500 \mathrm{~d}, \quad \mathrm{M}_{\mathrm{NO} 3(\mathrm{~L})} \mathrm{H}(\infty), \quad \mathrm{M}_{\mathrm{NO} 3(\mathrm{~S})} \mathrm{H} 500 \mathrm{~d}, \quad \mathrm{M}_{\mathrm{NO} 3(\mathrm{~S})} \mathrm{H}(\infty)$, $\mathrm{NI}_{\mathrm{NO} 3(\text { Alle) }} 500 \mathrm{~d}, \mathrm{VI}_{\mathrm{NO} 3(\text { Alle) }} 500 \mathrm{~d}, \mathrm{VI}_{\mathrm{NO} 3(\text { Alle) }}(\infty), \mathrm{NI}_{\mathrm{NO} 3(\mathrm{~L})} 500 \mathrm{~d}, \mathrm{VI}_{\mathrm{NO} 3(\mathrm{~L})} 500 \mathrm{~d}, \mathrm{VI}_{\mathrm{NO} 3(\mathrm{~L})}(\infty)$,

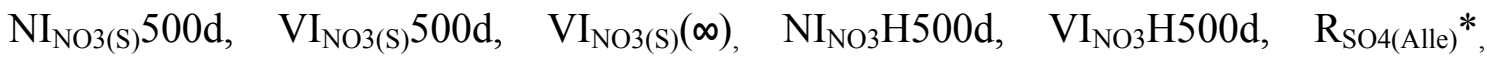
$\mathrm{M}_{\mathrm{SO} 4(\mathrm{Alle})} * 500 \mathrm{~d}, \quad \mathrm{M}_{\mathrm{SO} 4(\mathrm{Alll})} *(\infty), \quad \mathrm{M}_{\mathrm{SO} 4(\mathrm{Alle})} * \mathrm{~A} 500 \mathrm{~d}, \mathrm{M}_{\mathrm{SO} 4(\mathrm{~L})} \mathrm{A} 500 \mathrm{~d}, \mathrm{M}_{\mathrm{SO} 4(\mathrm{~S})} \mathrm{A} 500 \mathrm{~d}$,

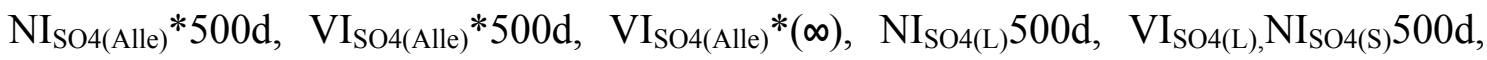
$\left.\mathrm{VI}_{\mathrm{SO} 4(\mathrm{~S})} 500 \mathrm{~d}, \mathrm{NI}_{\mathrm{SO} 4(\mathrm{~L})} \mathrm{A} 500 \mathrm{~d}, \mathrm{VI}_{\mathrm{SO} 4(\mathrm{~L})} \mathrm{A} 500 \mathrm{~d}, \mathrm{NI}_{\mathrm{SO} 4(\mathrm{~S})} \mathrm{A} 500 \mathrm{~d}, \mathrm{VI}_{\mathrm{SO} 4(\mathrm{~S})} \mathrm{A} 500 \mathrm{~d}\right)$ und allen unabhängigen Parametern $\left(\mathrm{C}_{\mathrm{org}}, \mathrm{C}_{\mathrm{hwl}}, \mathrm{C}_{\text {lös }}, \mathrm{C} / \mathrm{N}, \mathrm{CaCO}_{3}\right.$, Sulfid, pH, $\mathrm{Fe}_{\text {lös }}$, Sand-, Tonund Schluffgehalt). Für jeden der aufgeführten abhängigen Parameter wurde eine Regressionsgerade berechnet.

Zwei Regressionsverfahren wurden verwendet: „schrittweise“ und „direkt“. Bei dem „direkten“ Verfahren werden grundsätzlich alle unabhängigen Variablen verwendet. Beim „schrittweisen“ Verfahren wird die Auswahl der Parameter variiert, um nicht signifikante Größen zu eliminieren.

Tab. A 5 (im Anhang) gibt einen Überblick über die Ergebnisse der Regressionsgeraden zur Beschreibung der Beziehung zwischen den abhängigen und den unabhängigen Variablen. Dort sind die Ergebnisse der beiden verwendeten Verfahren (Schrittweise- und Direktverfahren) dargestellt.

In Tab. 10 sind die Ergebnisse der Regressionsanalyse für ausgewählte abhängige Variablen nach dem „schrittweisen“ Verfahren dargestellt. Dieses Verfahren wurde für die Modellbildung ausgewählt, weil durch die Elimination der unbedeutenden Variablen zum einen eine Prognose der abhängigen Variablen erleichtert wird und zum anderen die statistische Absicherung besser ist. Folgende Ergebnisse sind hervorzuheben:

Bei der Nitratreduktionsrate zu Versuchsbeginn $\left(\mathrm{R}_{\mathrm{NO} 3}\right)$ ergeben sich für die Regressionsgerade Bestimmtheitsmaße $\left(\mathrm{R}^{2}\right)$ von 0,24 (Auswahl alle Proben), 0,09 (Auswahl Sandböden) und 0,28 (Auswahl Lehmböden). D.h. hier lassen sich zwischen 9 und $28 \%$ der Varianzen durch die Regressionsgerade erklären. Die in der Regressionsgerade berücksichtigten Koeffizienten sind nur die Variablen $\mathrm{C}_{\text {org }}$ und Sulfid. 
Tab. 10a: Ergebnisse der linearen multiplen Regressionsanlyse zwischen unabhängigen und abhängigen Variablen, Koeffizienten der Regressionsgeraden mit statistischen Kenngrößen. Regressionsverfahren = schrittweise. Auswahl: Alle Porben

KOEFFIZIENTEN

STATISTIK

\begin{tabular}{|c|c|c|c|c|c|c|c|c|c|c|c|c|c|c|c|}
\hline Parameter & Konstante & $\begin{array}{c}\log C_{\text {org }} \\
\%\end{array}$ & DOC & $C_{\mathrm{hwl}}$ & $\begin{array}{l}\log N_{t} \\
\mathrm{mg} / \mathrm{kg}\end{array}$ & $\begin{array}{l}\text { Log } \\
\text { Sulfid }\end{array}$ & $\begin{array}{l}\log \\
F_{e_{\text {lös }}} \\
\end{array}$ & $\begin{array}{l}\text { pH } \\
\left(\mathrm{CaCl}_{2}\right)\end{array}$ & $\mathrm{CaCO}_{3}$ & Sand & $\begin{array}{r}\text { Log } \\
\text { Ton } \\
\%\end{array}$ & $\begin{array}{l}\text { Log } \\
\text { Schluff }\end{array}$ & $\mathbf{R}^{2}$ & $\mathbf{P}$ & $\mathbf{N}$ \\
\hline $\log \left[\mathrm{R}_{\mathrm{NO} \text { (Alle) }}\right]$ & $-1,01$ & 0,84 & & & & 0,20 & & & & & & & 0,24 & $1,0 \mathrm{E}-14$ & 233 \\
\hline $\mathrm{M}_{\mathrm{NO} \text { (Alle) }} 500 \mathrm{~d}$ & 120 & 243 & & & & 14,2 & & 27 & & & & & 0,63 & $9,9 E-50$ & 233 \\
\hline $\log \left[\mathrm{M}_{\mathrm{NO} \text { (Alle) }}(\infty)\right]$ & 0,40 & & & & 1,65 & 0,27 & & 0,35 & & 0,03 & & & 0,36 & $5,9 \mathrm{E}-22$ & 233 \\
\hline $\log \left[\mathrm{NI}_{\mathrm{NO} 3(\mathrm{Alle})} 500 \mathrm{~d}\right]$ & $-0,26$ & 1,20 & & & & 0,14 & & & & & $-0,93$ & & 0,21 & $2,4 \mathrm{E}-12$ & 233 \\
\hline $\mathrm{VI}_{\mathrm{NO}(\mathrm{Alle})} 500 \mathrm{~d}$ & $-0,02$ & $-0,01$ & & & & & & 0,01 & & & & & 0,10 & 1,5E-05 & 233 \\
\hline $\log \left[\mathrm{VI}_{\text {NO3(Alle) }}(\infty)\right]$ & $-1,84$ & & & & & 0,12 & & & & & & & 0,07 & 3,2E-05 & 233 \\
\hline $\log \left[\mathrm{R}_{\mathrm{SO}(\text { Alle })^{*}}\right]$ & $-1,44$ & & & & & 0,25 & & 0,09 & & & & & 0,29 & 7,0E-09 & 108 \\
\hline $\mathrm{M}_{\mathrm{SO} 4 \text { (Alle) }}{ }^{*} 500 \mathrm{~d}$ & 22,5 & 106 & $-1,90$ & & & 68,0 & & & & & & & 0,44 & $1,0 \mathrm{E}-13$ & 108 \\
\hline $\mathrm{M}_{\mathrm{SO} 4(\mathrm{Alll}))^{*}(\infty)}$ & 56,4 & 133 & $-2,46$ & & & 44,3 & & & & & & & 0,44 & $1,1 \mathrm{E}-13$ & 108 \\
\hline $\mathrm{NI}_{\mathrm{SO} 4 \text { (Alle) }}{ }^{*} 500 \mathrm{~d}$ & 0,20 & 0,11 & & & & & & & $-0,01$ & & & & 0,12 & 4,4E-04 & 108 \\
\hline $\mathrm{VI}_{\text {SO4(Alle) }}{ }^{*} 500 \mathrm{~d}$ & 0,36 & & & & & 0,0 & & & $-0,03$ & & & & 0,09 & 1,2E-03 & 108 \\
\hline $\mathrm{VI}_{\text {SO4(Alle) }}{ }^{*}(\infty)$ & 0,25 & & & & & 0,0 & & $-0,05$ & $-0,02$ & & & 0,31 & 0,26 & 1,7E-07 & 108 \\
\hline $\log \left[\mathrm{R}_{\mathrm{SO}(\text { Alle) }}\right]^{*}$ & $-2,36$ & & & & & 0,41 & & 0,14 & & & & & 0,29 & $7,0 \mathrm{E}-09$ & 108 \\
\hline $\mathrm{MSO}_{\text {(Alle) }}{ }^{*} 500 \mathrm{~d}$ & 37 & 175 & $-3,12$ & & & 112 & & & & & & & 0,44 & $1,0 \mathrm{E}-13$ & 108 \\
\hline $\mathrm{M}_{\mathrm{SO} 4(\mathrm{Alll}))^{*}(\infty)}$ & 92 & 219 & $-4,03$ & & & 73 & & & & & & & 0,44 & $1,1 \mathrm{E}-13$ & 108 \\
\hline $\mathrm{NI}_{\text {SO4(Alle) }}{ }^{*} 500 \mathrm{~d}$ & 0,32 & 0,18 & & & & & & & $-0,02$ & & & & 0,12 & 4,4E-04 & 108 \\
\hline $\mathrm{VI}_{\mathrm{SO} 4 \text { (Alle) }} * 500 \mathrm{~d}$ & 0,60 & & & & & & & & $-0,04$ & & & & 0,09 & 1,2E-03 & 108 \\
\hline $\mathrm{VI}_{\mathrm{SO} 4(\text { Alle })^{*}(\infty)}$ & 0,42 & & & & & & & $-0,08$ & $-0,03$ & & & 0,31 & 0,26 & 1,7E-07 & 108 \\
\hline $\log \left[R_{\mathrm{NO} 3(\mathrm{~S})}\right]$ & $-1,45$ & & & & & 0,19 & & & & & $-1,03$ & & 0,09 & 1,0E-03 & 123 \\
\hline $\mathrm{M}_{\mathrm{NO} 3(\mathrm{~S})} 500 \mathrm{~d}$ & 95 & 48 & $-0,88$ & & & 4,15 & & & 205 & & & & 0,25 & 8,1E-08 & 123 \\
\hline $\log \left[\mathrm{M}_{\mathrm{NO}(\mathrm{S})}(\infty)\right]$ & $-1,90$ & & & & & 0,34 & & 0,60 & & & & & 0,16 & $9,9 \mathrm{E}-06$ & 123 \\
\hline $\log \left[\mathrm{NI}_{\mathrm{NO}(\mathrm{S})} 500 \mathrm{~d}\right]$ & $-1,51$ & & & & & 0,23 & & & & & & & 0,06 & 4,0E-03 & 123 \\
\hline $\log \left[\mathrm{VI} \mathrm{N}_{\mathrm{NO} 3(\mathrm{~S})} 500 \mathrm{~d}\right]$ & $-3,99$ & & & & & 0,12 & & 0,44 & & & & & 0,11 & 2,7E-04 & 123 \\
\hline
\end{tabular}


Tab. 10b (Fortsetzung): Ergebnisse der linearen multiplen Regressionsanalyse zwischen unabhängigen und abhängigen Variablen, Koeffizienten der Regressionsgeraden mit statistischen Kenngrößen. Regressionsverfahren = schrittweise. Auswahl: Lehmböden

KOEFFIZIENTEN STATISTIK -

\begin{tabular}{|c|c|c|c|c|c|c|c|c|c|c|c|c|c|c|c|}
\hline Parameter & Konstante & $\begin{array}{c}\log C_{\text {org }} \\
\%\end{array}$ & DOC & $\mathbf{C}_{\text {hwl }}$ & $\frac{\log N_{t}}{\mathrm{mg} / \mathrm{kq}}$ & $\begin{array}{l}\text { Log } \\
\text { Sulfid }\end{array}$ & $\begin{array}{l}\text { Log } \\
F_{\text {lös }}\end{array}$ & $\begin{array}{l}\mathrm{pH} \\
\left(\mathrm{CaCl}_{2}\right)\end{array}$ & $\mathrm{CaCO}_{3}$ & $\begin{array}{c}\text { Sand } \\
\%\end{array}$ & $\begin{array}{l}\text { Log } \\
\text { Ton }\end{array}$ & $\begin{array}{l}\text { Log } \\
\text { Schluff }\end{array}$ & $\mathbf{R}^{2}$ & $\mathbf{P}$ & $\mathbf{N}$ \\
\hline $\log \left[\mathrm{VI} \mathrm{NO}_{\mathrm{NO}(\mathrm{S})}(\infty)\right]$ & $-3,63$ & & & & & 0,13 & & 0,38 & & & & & 0,12 & $1,5 \mathrm{E}-04$ & 123 \\
\hline $\log \left[\mathrm{R}_{\mathrm{NO}(\mathrm{L})}\right]$ & $-1,26$ & 0,81 & & & & 0,32 & & & & & & & 0,28 & 9,9E-09 & 110 \\
\hline $\mathrm{M}_{\mathrm{NO} 3(\mathrm{~L})} 500 \mathrm{~d}$ & 245 & 270 & & & & 41 & & & & & & & 0,62 & 1,3E-23 & 110 \\
\hline $\log \left[\mathrm{M}_{\mathrm{NO} 3(\mathrm{~L})}(\infty)\right]$ & 9,22 & & & & 2,33 & & & 0,56 & & & & $-4,69$ & 0,51 & $4,6 \mathrm{E}-17$ & 110 \\
\hline $\log \left[\mathrm{NI}_{\mathrm{NO} 3(\mathrm{~L})} 500 \mathrm{~d}\right]$ & $-3,26$ & 1,76 & & & & & & 0,34 & & & & & 0,42 & $7,4 \mathrm{E}-14$ & 110 \\
\hline $\log \left[\mathrm{VI}_{\mathrm{NO}(\mathrm{L})} 500 \mathrm{~d}\right]$ & $-1,07$ & & & & 0,63 & & & & & & & & 0,24 & 3,8E-08 & 110 \\
\hline $\log \left[V I_{\mathrm{NO}(\mathrm{L})}(\infty)\right]$ & $-1,92$ & & & & 0,51 & & & 0,14 & & & & & 0,20 & $2,4 \mathrm{E}-06$ & 110 \\
\hline $\log \left[\mathrm{R}_{\mathrm{SO}(\mathrm{L})}\right]$ & $-1,23$ & & & & & 0,39 & & & & & & & 0,36 & $1,2 \mathrm{E}-08$ & 73 \\
\hline $\mathrm{M}_{\mathrm{SO} 4(\mathrm{~L})} 500 \mathrm{~d}$ & -466 & 261 & & & -227 & 166 & & & $-16,9$ & & & & 0,60 & $1,2 \mathrm{E}-13$ & 73 \\
\hline $\mathrm{M}_{\mathrm{SO} 4(\mathrm{~L})}(\infty)$ & -437 & 282 & & & -224 & 144 & & & $-19,8$ & & & & 0,58 & $5,6 \mathrm{E}-13$ & 73 \\
\hline $\mathrm{NI}_{\mathrm{SO} 4(\mathrm{~L})} 500 \mathrm{~d}$ & 1,46 & & & & & & & & & & & $-1,24$ & 0,28 & $7,4 \mathrm{E}-07$ & 73 \\
\hline $\mathrm{VI}_{\mathrm{SO}(\mathrm{L})} 500 \mathrm{~d}$ & 0,38 & & & & & & & & $-0,03$ & & & & 0,12 & 1,3E-03 & 73 \\
\hline $\mathrm{VI}_{\mathrm{SO} 4(\mathrm{~L})}(\infty)$ & 0,99 & & & & & $-0,10$ & & $-0,08$ & & & & & 0,36 & $5,2 \mathrm{E}-08$ & 73 \\
\hline $\log \left[\mathrm{R}_{\mathrm{SO}(\mathrm{S})}\right]$ & $-0,44$ & & $-0,01$ & & & & & & & & & & 0,05 & 1,0E-01 & 35 \\
\hline $\mathrm{M}_{\mathrm{SO} 4(\mathrm{~S})} 500 \mathrm{~d}$ & 95,6 & & $-1,14$ & & & 11,2 & & & 175 & & -127 & $-75,5$ & 0,81 & $1,1 \mathrm{E}-10$ & 35 \\
\hline $\mathrm{M}_{\mathrm{SO} 4(\mathrm{~S})}(\infty)$ & $-35,8$ & & & & & 3,50 & & 8,37 & & & & & 0,30 & $1,2 \mathrm{E}-03$ & 35 \\
\hline $\mathrm{NI}_{\mathrm{SO}(\mathrm{S})} 500 \mathrm{~d}$ & 0,33 & & & & & $-0,07$ & & & & & & $-0,21$ & 0,25 & 3,9E-03 & 35 \\
\hline $\mathrm{VI}_{\mathrm{SO} 4(\mathrm{~S})} 500 \mathrm{~d}$ & $-1,31$ & & $-0,01$ & & & 0,09 & & 0,33 & & & & & 0,66 & 5,8E-08 & 35 \\
\hline $\mathrm{VI}_{\mathrm{SO} 4(\mathrm{~S})}(\infty)$ & 0,86 & & & & & & & & & $-0,01$ & & & 0,28 & $7,2 \mathrm{E}-04$ & 35 \\
\hline $\log \left[\mathrm{M}_{\text {SO4(Alle) }} \mathrm{A} 500 \mathrm{~d}\right]$ & 0,12 & & & & & 0,50 & 0,14 & & & & & & 0,23 & $4,5 \mathrm{E}-06$ & 88 \\
\hline $\mathrm{VI}_{\mathrm{SO} 4(\mathrm{Alle})} \mathrm{A} 500 \mathrm{~d}$ & 0,37 & & & & & 0,07 & & & & & & $-0,23$ & 0,10 & 3,4E-03 & 88 \\
\hline $\log \left[\mathrm{M}_{\mathrm{SO} 4(\mathrm{~L})} \mathrm{A} 500 \mathrm{~d}\right]$ & $-4,48$ & 5,45 & & & $-4,29$ & & & & 0,28 & & & & 0,69 & $5,6 \mathrm{E}-13$ & 35 \\
\hline
\end{tabular}


Tab. 10C (Fortsetzung): Ergebnisse der linearen multiplen Regressionsanalyse zwischen unabhängigen und abhängigen Variablen, Koeffizienten der Regressionsgeraden mit statistischen Kenngrößen. Regressionsverfahren = schrittweise. Auswahl: Sandböden

KOEFFIZIENTEN STATISTIK

\begin{tabular}{|c|c|c|c|c|c|c|c|c|c|c|c|c|c|c|c|}
\hline Parameter & Konstante & $\begin{array}{c}\log C_{\text {org }} \\
\%\end{array}$ & DOC & $C_{h w l}$ & $\begin{array}{l}\log N_{t} \\
\mathrm{mg} / \mathrm{kg}\end{array}$ & $\begin{array}{l}\text { Log } \\
\text { Sulfid }\end{array}$ & $\begin{array}{l}\text { Log } \\
F_{\text {lös }} \\
\end{array}$ & $\begin{array}{l}\mathrm{pH} \\
\left(\mathrm{CaCl}_{2}\right)\end{array}$ & $\mathrm{CaCO}_{3}$ & Sand & $\begin{array}{l}\text { Log } \\
\text { Ton }\end{array}$ & $\begin{array}{l}\text { Log } \\
\text { Schluff }\end{array}$ & $\mathbf{R}^{2}$ & $\mathbf{P}$ & $\mathbf{N}$ \\
\hline $\mathrm{VI}_{\mathrm{SO} 4(\mathrm{~L})} \mathrm{A} 500 \mathrm{~d}$ & 2,39 & 0,18 & & & & 0,00 & & & & $-0,01$ & & $-1,86$ & 0,32 & 5,7E-05 & 35 \\
\hline $\log \left[\mathrm{M}_{\mathrm{SO} 4(\mathrm{~S})} \mathrm{A} 500 \mathrm{~d}\right]$ & 0,58 & 0,00 & $-0,01$ & & 0,13 & 0,11 & & 0,20 & & & & $-0,26$ & 0,84 & $1,5 \mathrm{E}-11$ & 35 \\
\hline $\mathrm{VI}_{\mathrm{SO} 4(\mathrm{~S})} \mathrm{A} 500 \mathrm{~d}$ & $-0,48$ & & $-0,01$ & & & 0,09 & & 0,17 & & & & & 0,38 & 4,2E-04 & 35 \\
\hline $\log \left[\mathrm{R}_{\mathrm{SO} 4(\mathrm{~L})}\right]$ & $-2,01$ & & & & & 0,64 & & & & & & & 0,36 & $1,2 \mathrm{E}-08$ & 73 \\
\hline $\mathrm{M}_{\mathrm{SO} 4(\llcorner) 500 \mathrm{~d}}$ & -764 & 428 & & & -373 & 272 & & & -26 & & & & 0,60 & $1,2 \mathrm{E}-13$ & 73 \\
\hline $\mathrm{MSO} 4(\mathrm{~L})_{(\infty)}$ & -717 & 462 & & & -367 & 236 & & & -33 & & & & 0,58 & $5,6 \mathrm{E}-13$ & 73 \\
\hline $\mathrm{NI}_{\mathrm{SO} 4(L)} 500 \mathrm{~d}$ & 2,40 & & & & & & & & & & & $-1,24$ & 0,28 & $7,4 \mathrm{E}-07$ & 73 \\
\hline $\mathrm{VI}_{\mathrm{SO} 4(\mathrm{~L})} 500 \mathrm{~d}$ & 0,62 & & & & & & & & $-0,05$ & & & & 0,12 & 1,3E-03 & 73 \\
\hline $\mathrm{VI}_{\mathrm{SO} 4(\mathrm{~L})}(\infty)$ & 1,63 & & & & & $-0,17$ & & $-0,14$ & & & & & 0,36 & $5,2 \mathrm{E}-08$ & 73 \\
\hline $\log \left[\mathrm{R}_{\mathrm{SO} 4(\mathrm{~S})}\right]$ & $-0,72$ & & $-0,02$ & & & & & & & & & & 0,05 & 1,0E-01 & 35 \\
\hline $\mathrm{M}_{\mathrm{SO} 4(\mathrm{~S})} 500 \mathrm{~d}$ & 157 & & $-1,87$ & & & 18 & & & 287 & & -210 & -76 & 0,81 & $1,1 \mathrm{E}-10$ & 35 \\
\hline $\mathrm{M}_{\mathrm{SO} 4(\mathrm{~S})}(\infty)$ & -59 & & & & & 5,74 & & 14 & & & & & 0,30 & $1,2 \mathrm{E}-03$ & 35 \\
\hline $\mathrm{NI}_{\mathrm{SO} 4(\mathrm{~S})} 500 \mathrm{~d}$ & 0,55 & & & & & $-0,11$ & & & & & & $-0,21$ & 0,25 & 3,9E-03 & 35 \\
\hline $\mathrm{VI}_{\mathrm{SO} 4(\mathrm{~S})} 500 \mathrm{~d}$ & $-2,14$ & & $-0,01$ & & & 0,14 & & 0,54 & & & & & 0,66 & $5,8 \mathrm{E}-08$ & 35 \\
\hline $\mathrm{VI}_{\mathrm{SO} 4(\mathrm{~S})}(\infty)$ & 1,41 & & & & & & & & & $-0,01$ & & & 0,28 & 7,2E-04 & 35 \\
\hline $\begin{array}{l}\log \\
{\left[M_{\text {SO4(Alle) }} \text { A500d] }\right.}\end{array}$ & 0,20 & & & & & 0,82 & 0,23 & & & & & & 0,23 & 4,5E-06 & 88 \\
\hline $\mathrm{VI}_{\mathrm{SO} 4(\mathrm{Alle})} \mathrm{A} 500 \mathrm{~d}$ & 0,61 & & & & & 0,11 & & & & & & $-0,23$ & 0,10 & $3,4 \mathrm{E}-03$ & 88 \\
\hline $\log \left[\mathrm{M}_{\mathrm{SO} 4(\mathrm{~L})} \mathrm{A} 500 \mathrm{~d}\right]$ & $-7,34$ & 8,93 & & & $-7,04$ & & & & 0,45 & & & & 0,69 & $5,6 \mathrm{E}-13$ & 53 \\
\hline $\mathrm{VI}_{\mathrm{SO} 4(\mathrm{~L})} \mathrm{A} 500 \mathrm{~d}$ & 3,93 & 0,30 & & & & & & & & $-0,01$ & & $-1,86$ & 0,32 & 5,7E-05 & 53 \\
\hline $\log \left[\mathrm{M}_{\mathrm{SO} 4(\mathrm{~S})} \mathrm{A} 500 \mathrm{~d}\right]$ & 0,95 & & $-0,02$ & & 0,21 & 0,18 & & 0,32 & & & & $-0,26$ & 0,84 & $1,5 \mathrm{E}-11$ & 35 \\
\hline $\mathrm{VI}_{\mathrm{SO} 4(\mathrm{~S})} \mathrm{A} 500 \mathrm{~d}$ & $-0,79$ & & $-0,01$ & & & 0,15 & & 0,27 & & & & & 0,38 & 4,2E-04 & 35 \\
\hline
\end{tabular}


Tab. 10 (Fortsetzung): Ergebnisse der linearen multiplen Regressionsanalyse zwischen unabhängigen und abhängigen Variablen, Koeffizienten der Regressionsgeraden mit statistischen Kenngrößen. Regressionsverfahren = schrittweise

KOEFFIZIENTEN STATISTIK

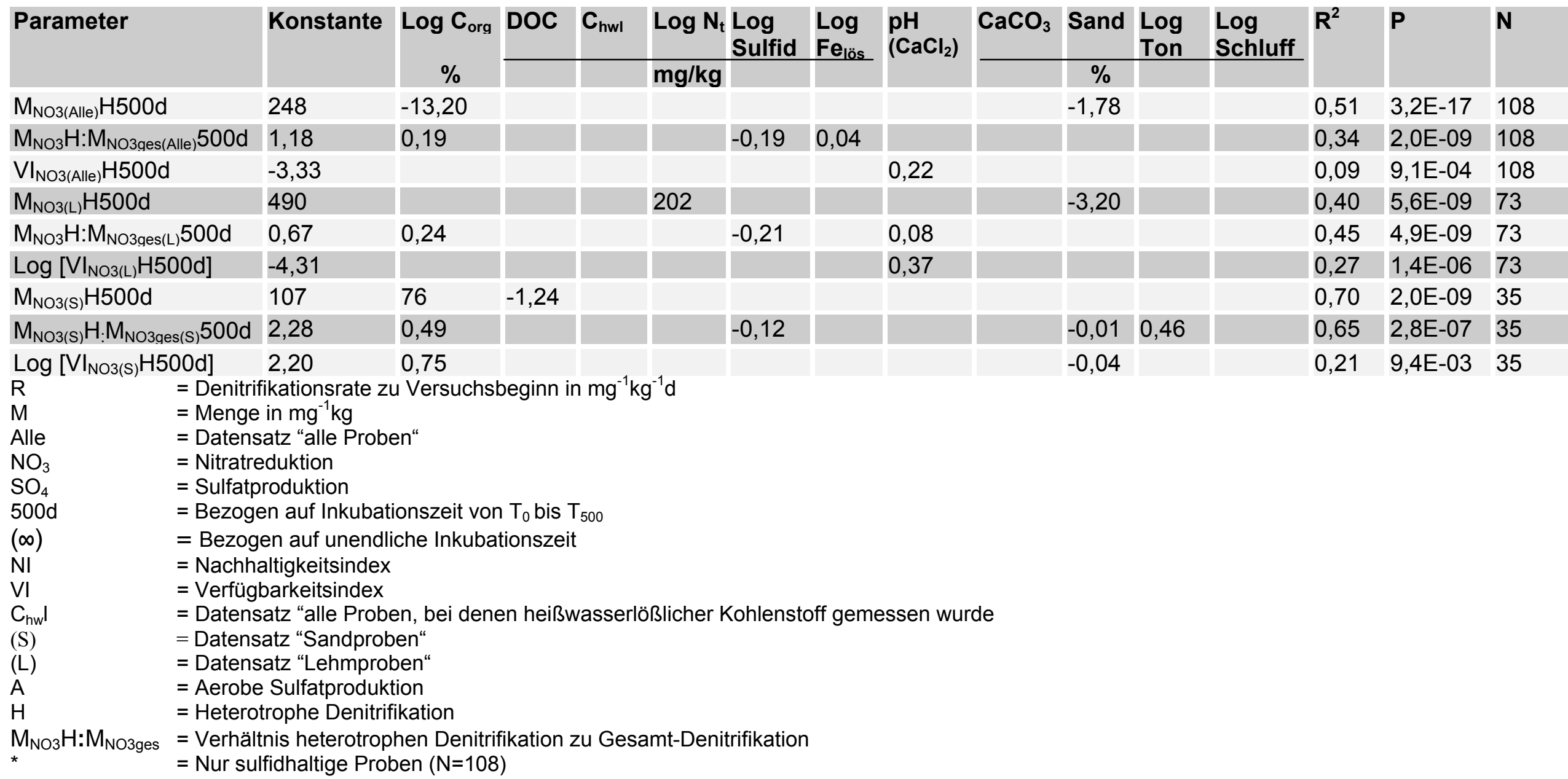


Für die Messgröße $\mathrm{M}_{\mathrm{NO} 3}$ 500d betrug $\mathrm{R}^{2}$ für die Auswahl “Alle“ 0,63, für die Auswahl "Sand" 0,25 und für die Auswahl "Lehm" 0,60. Die kumulierte Nitratreduktion bezogen auf die unendliche Inkubationszeit $\left(\mathrm{M}_{\mathrm{NO} 3}(\infty)\right)$ ergab für jede Datenauswahl ein geringeres Bestimmtheitsmaß $\left(\mathrm{R}^{2}=0,36\right.$ für die Auswahl "Alle“, $\mathrm{R}^{2}=0,16$ für die Auswahl "Sand", $\mathrm{R}^{2}=0,58$ für die Auswahl "Lehm").

Für die kumulierte Nitratreduktion durch heterotrophe Denitrifikation $\left(\mathrm{M}_{\mathrm{NO}} 500 \mathrm{dH}\right)$ ergeben sich im Vergleich zu $\mathrm{M}_{\mathrm{NO} 3} 500 \mathrm{~d}$ noch höhere Werte für $\mathrm{R}^{2}(0,51$ für die Auswahl "Alle", 0,70 für die Auswahl "Sand", 0,40 für die Auswahl "Lehm").

Bei allen Variablen der kumulierten Nitratreduktion $\left(\mathrm{M}_{\mathrm{NO} 3}\right)$ gehen die Koeffizienten der Parameter des Reduktionsmittelvorrats in die Regressionsgerade ein. Dabei ist Sulfid in jedem Fall vertreten; der organische Kohlenstoff wird entweder als $\mathrm{C}_{\text {org }}$ oder als $\mathrm{N}_{\mathrm{t}}$ berücksichtigt. Die Koeffizienten für $\mathrm{pH}, \mathrm{CaCO}_{3}$ und Textur sind nur teilweise vertreten.

Die Nachhaltigkeitsindizes für die Nitratreduktion bis 500 Tage nach $\mathrm{T}_{0}\left(\mathrm{NI}_{\mathrm{NO} 3} 500 \mathrm{~d}\right)$ lassen für die Auswahl "Alle" mit $\mathrm{R}^{2}=0,21$ befriedigende, für die Auswahl "Sand" mit $\mathrm{R}^{2}=0,06$ sehr geringe und für die Auswahl "Lehm" mit $\mathrm{R}^{2}=0,42$ relativ gute Vorhersagen treffen. Folgende Parameter gehen hier als Koeffizienten in die Regressionsgerade ein: für die Auswahl "Alle" $\mathrm{C}_{\text {org }}$, Sulfid und Ton, für die Auswahl "Sand" Sulfid und für die Auswahl "Lehm" $\mathrm{C}_{\text {org }}$ und $\mathrm{pH}-$ Wert.

Für die Verfügbarkeitsindizes der Nitratreduktion $\left(\mathrm{VI}_{\mathrm{NO} 3} 500 \mathrm{~d}, \mathrm{VI}_{\mathrm{NO} 3}(\infty)\right)$ ergaben sich nur geringe Bestimmtheitsmaße $\left(\mathrm{R}^{2}=0,07\right.$ bis 0,24$)$. Die bei $\mathrm{VI}_{\mathrm{NO}_{3}} 500 \mathrm{~d}$ berücksichtigten Koeffizienten sind $\mathrm{C}_{\text {org }}$ und $\mathrm{pH}$ bei der Auswahl "Alle“, Sulfid und $\mathrm{pH}$ bei der Auswahl "Sand" und bei der Auswahl "Lehm" ist es der $\mathrm{N}_{\mathrm{t}}-$ Gehalt. Bei $\mathrm{VI}_{\mathrm{NO} 3}(\infty)$ gehen folgende Koeffizienten ein: Sulfid bei der Auswahl “Alle“, ebenfalls Sulfid und zusätzlich $\mathrm{pH}$ bei der Auswahl "Sand" sowie $\mathrm{N}_{\mathrm{t}}$ und $\mathrm{pH}$ bei der Auswahl "Lehm".

Bei der anaeroben Sulfatproduktionsrate $\left(\mathrm{R}_{\mathrm{SO} 4}\right)$ beträgt $\mathrm{R}^{2}$ 0,29 für die Auswahl "Alle“, 0,09 für die Auswahl "Sand" und 0,36 für die Auswahl "Lehm" und ist somit nur mäßig vorhersagbar. Als Koeffizient für die Regressionsgerade ist hier in jedem Fall Sulfid vertreten. Bei der Auswahl "Alle“ kommt der pH hinzu, bei den Datensätzen "Sand" und "Lehm" kommen noch Texturparameter hinzu.

Die kumulierte anaerobe Sulfatproduktion bis 500 Tage nach $\mathrm{T}_{0}\left(\mathrm{M}_{\mathrm{SO} 4} 500 \mathrm{~d}\right)$ lässt sich vergleichsweise gut modellieren: das Bestimmtheitsmaß beträgt für die jeweiligen ausgewählten Datensätze 0,44 (,Alle“), 0,81 (,Sand“) und 0,60 („Lehm“). Sulfid geht in jedem Fall als Koeffizient in die Regressionsgerade ein. Darüber hinaus werden folgende Variablen berücksichtigt: für die Auswahl "Alle" $\mathrm{C}_{\text {org }}$ und $\mathrm{C}_{\text {lös }}$ (mit negativem Vor- 
zeichen), für die Auswahl "Sand" $\mathrm{CaCO}_{3}$ und die Parameter $\mathrm{C}_{\text {lös, }}$ Ton und Schluff mit negativem Vorzeichen, für die Auswahl "Lehm" $\mathrm{C}_{\mathrm{org}}, \mathrm{N}_{\mathrm{t}}$ und $\mathrm{CaCO}_{3}$ (mit negativem Vorzeichen). Für die kumulierte Sulfatproduktion bezogen auf die unendliche Inkubationszeit $\left(\mathrm{M}_{\mathrm{SO} 4}(\infty)\right)$ ist die Prognose nicht ganz so gut $\left(\mathrm{R}^{2}=0,16\right.$ bis 0,58$)$.

Der Nachhaltigkeitsindex der Sulfatproduktion bis 500 Tage nach $\mathrm{T}_{0}\left(\mathrm{NI}_{\mathrm{SO}} 500 \mathrm{~d}\right)$ ist nicht gut vorhersagbar ( $\mathrm{R}^{2}=0,12$ für "Alle“, 0,25 für "Sand" und 0,28 für "Lehm“).

Der Verfügbarkeitsindex für die kumulierte Sulfatproduktion bis 500 Tage nach $\mathrm{T}_{0}$ (VI ${ }_{\mathrm{SO} 4} 500 \mathrm{~d}$ ) ergibt für die Datenauswahl "Alle“ $\mathrm{R}^{2}=0,09$, für "Sand" $\mathrm{R}^{2}=0,66$ und für “Lehm" $\mathrm{R}^{2}=0,12$. Die Güte der Prognose weist damit einen breiten Schwankungsbereich auf. Bei dem Verfügbarkeitsindex für die kumulierte Sulfatproduktion bezogen auf die unendliche Inkubationszeit $\left(\mathrm{VI}_{\mathrm{SO} 4}(\infty)\right)$ ist $\mathrm{R}^{2}$ für die verschiedenen Datensätze eher einheitlich (“Alle“ mit $\mathrm{R}^{2}=0,26$, "Sand" mit $\mathrm{R}^{2}=0,28$, "Lehm" mit $\mathrm{R}^{2}=0,36$ ).

Für die kumulierte aerobe Sulfatproduktion $\left(\mathrm{M}_{\mathrm{SO} 4} \mathrm{~A}\right)$ lässt sich folgendes festhalten. $\mathrm{M}_{\mathrm{SO} 4} \mathrm{~A} 500 \mathrm{~d}$ ergibt gute Werte für die Teildatensätze „Sand“ und „Lehm“ $\left(\mathrm{R}^{2}=0,84\right.$ und 0,69). Wie bei der anaeroben Sulfatproduktion ist Sulfid immer als Variablenkoeffizient vertreten. Darüber hinaus werden teilweise die Koeffizienten für $\mathrm{Fe}_{\text {lös, }} \mathrm{C}_{\text {lös, }}$, $\mathrm{C}_{\text {org }}, \mathrm{N}_{\mathrm{t}}$, und $\mathrm{CaCO}_{3}$ berücksichtigt.

Bei dem Verfügbarkeitsindex der aeroben Sulfatproduktion ( $\left.\mathrm{VI}_{\mathrm{SO} 4} \mathrm{~A}\right)$ bewegt sich das Bestimmtheitsmaß zwischen 0,10 und 0,38 und ist somit in der gleichen Größenordnung wie beim Verfügbarkeitsindex der anaeroben Sulfatproduktion.

Zusammenzufassend lässt sich folgendes feststellen:

- Die Vorhersagbarkeit der abhängigen Messgrößen ist insgesamt recht unterschiedlich.

- Generell ließen sich die auf die Versuchsperiode (500 Tage nach $\mathrm{T}_{0}$ ) bezogenen Messgrößen besser abbilden als die auf unendliche Inkubationszeit $(\infty)$ extrapolierten Umsatzmengen.

- Die kumulierten Nitratreduktionen bis zu 500 Tagen nach $T_{0}$ lässt sich gut modellieren. Die kumulierte Sulfatproduktionen (aerob und anaerob) lassen sich am besten modellieren.

- Die Nachhaltigkeit der Denitrifikation und die Verfügbarkeit der Reduktionsmittelvorräte lassen sich nur schlecht vorhersagen.

- Teildatensätze liefern in vielen Fällen, aber nicht immer bessere Prognosen als der gesamte Datensatz. 
- $\mathrm{C}_{\text {org }}$ und Sulfid sind die wichtigsten Parameter für alle abhängigen Variablen. Sie gehen als Koeffizienten in fast alle der ermittelten Regressionsmodelle ein. Die Koeffizienten für $\mathrm{pH}, \mathrm{CaCO}_{3}$ und Textur werden nur teilweise berücksichtigt.

\subsubsection{Vergleich zwischen den gemessenen und den berechneten Werten}

Mit den in Tab. 10 aufgeführten Regressionsergebnissen wurden für die verschiedenen Messgrößen Werte berechnet. Tab. 11 zeigt den Vergleich zwischen den gemessenen und den mit den Regressionsmodellen berechneten Werten. Die Flächenmittelwerte für die berechneten Werte sind in \% der Messwerte dargestellt. Zusätzlich sind die absoluten Differenzen zu den Messwerten aufgeführt. Nach der Berechnung der Einzelwerte wurden für jede Messgröße Flächenmittelwerte gebildet. Aus diesen wurden dann Relativwerte sowie Differenzen abgeleitet.

Die berechneten Werte basieren auf den verschiedenen Datensätzen, die bei den Regressionsrechnungen verwendet wurden (vgl. Tab. 10). Zunächst wurden die Koeffizienten der Datenauswahl "Alle“ verwendet. Zusätzlich erfolgte eine differenzierte Berechnung (in Tab. 11 mit "Diff." gekennzeichnet), bei der die Koeffizienten der Datenauswahl "Sand" für die Sandproben und die der Datenauswahl "Lehm" für die Lehmproben herangezogen wurden.

Als Maß für die Übereinstimmung berechneter und gemessener Werte wurde die Korrelation zwischen den Mittelwerten berechneter und gemessener Werte als Bestimmtheitsmaß $\left(\mathrm{R}^{2}\right)$ aufgeführt.

Für einige Messgrößen sind in Abb. 8 exemplarisch die berechneten Werte in Relation zum Messwert dargestellt und zwar sowohl für die Einzelwerte als auch für die Flächenmittelwerte. Aus den Grafiken (Abb. 8) geht hervor, dass die Streuung für die Einzelwerte sehr groß ist. Es ist ersichtlich, dass sich Einzelwerte grundsätzlich nur unbefriedigend vorhersagen lassen. Bei den Flächenmittelwerten ist in vielen Fällen jedoch eine recht gute Übereinstimmung erzielt worden.

Tab. 11 liefert den Überblick für den Vergleich zwischen berechneten und gemessenen Flächenmittelwerten für alle Messgrößen. Eine insgesamt gute Übereinstimmung $\left(\mathrm{R}^{2}>\right.$ 0,90 ) ergibt sich für $\mathrm{M}_{\mathrm{NO} 3} 500 \mathrm{~d}, \mathrm{M}_{\mathrm{SO} 4} 500 \mathrm{~d}$ und $\mathrm{VI}_{\mathrm{SO} 4} 500 \mathrm{~d}$. Dabei ergeben die Variablen, bei denen nach Sand- und Lehmböden differenziert wurde, jeweils die beste Anpassung. 
Tab. 11: Flächenmittelwerte der berechneten Messgrößen in \% der Messwerte; absolute Differenzen zwischen gemessenen und berechnetenwerten in Klammern; $\mathbf{R}^{\mathbf{2}}=$ Bestimmtheitsmaß für die Korrelation zwischen gemessenen und berechneten Flächenmittelwerten

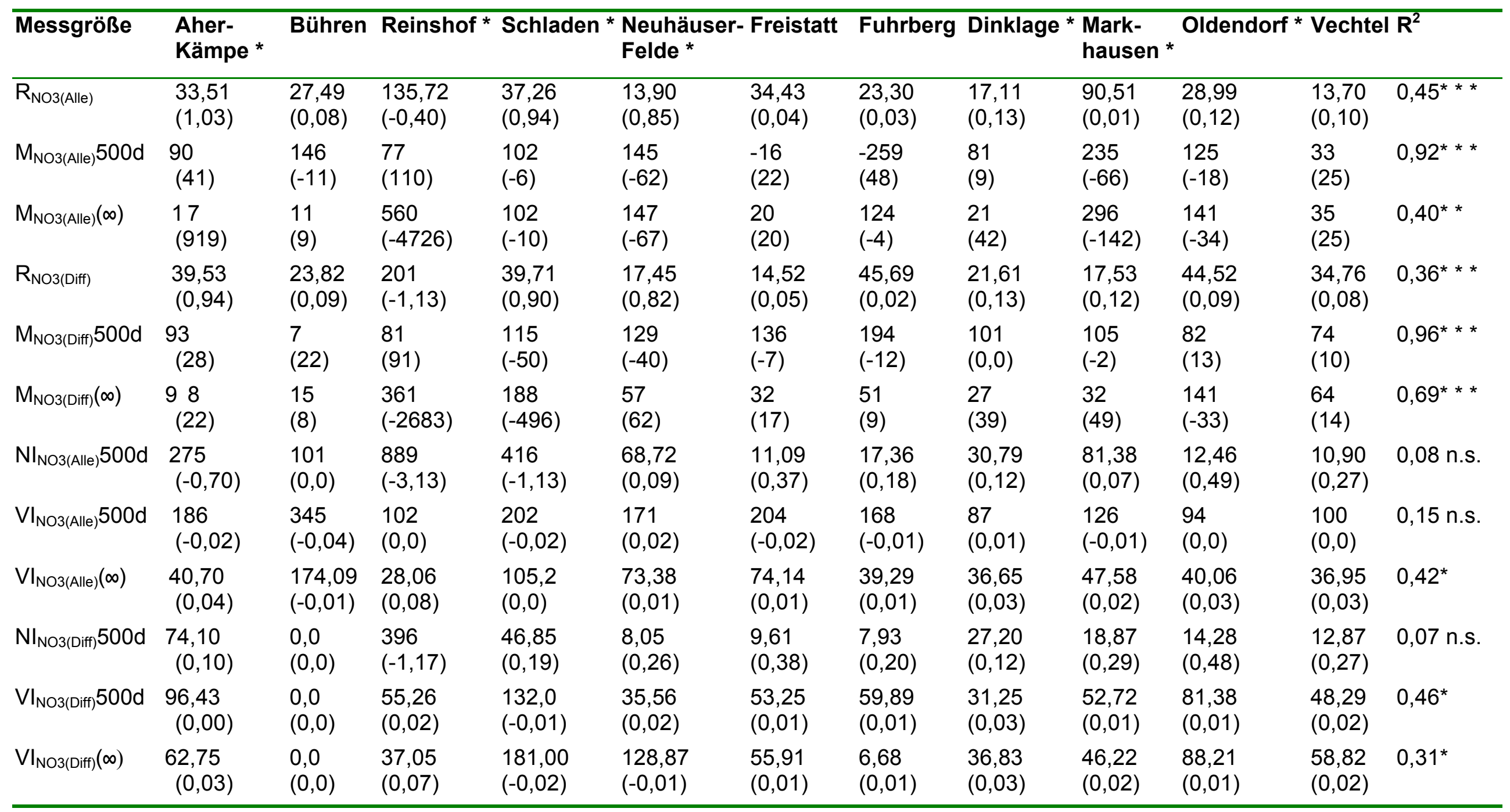


Tab. 11 (Fortsetzung ): Flächenmittelwerte der berechneten Messgrößen in \% der Messwerte; absolute Differenzen zwischen gemessenen und berechneten Werte in Klammern; $\mathbf{R}^{2}=$ Bestimmtheitsmaß für die Korrelation zwischen gemessenen und berechneten Flächenmittelwerten

\begin{tabular}{|c|c|c|c|c|c|c|c|c|c|c|c|c|}
\hline Messgröße & $\begin{array}{l}\text { Aher } \\
\text { Kämpe * }\end{array}$ & Bühren & Reinshof * & Schladen * & $\begin{array}{l}\text { Neuhäuser } \\
\text { Felde * }\end{array}$ & Freistatt & Fuhrberg & Dinklage * & $\begin{array}{l}\text { Mark- } \\
\text { Hausen * }\end{array}$ & Oldendorf * & Vechtel & $\mathbf{R}^{2}$ \\
\hline $\mathrm{M}_{\mathrm{SO} 4 \text { (Alle) }} 500 \mathrm{~d}$ & $\begin{array}{l}124 \\
(-45)\end{array}$ & $\begin{array}{l}0,0 \\
(0,0)\end{array}$ & $\begin{array}{l}110 \\
(-29)\end{array}$ & $\begin{array}{l}88 \\
(36)\end{array}$ & $\begin{array}{l}90 \\
(21)\end{array}$ & $\begin{array}{l}0,0 \\
(0,0)\end{array}$ & $\begin{array}{l}0,0 \\
(0,0)\end{array}$ & $\begin{array}{l}198 \\
(-12)\end{array}$ & $\begin{array}{l}417 \\
(-91)\end{array}$ & $\begin{array}{l}33 \\
(80)\end{array}$ & $\begin{array}{l}0,0 \\
(0,0)\end{array}$ & $0,87 * * *$ \\
\hline $\mathrm{M}_{\mathrm{SO} 4 \text { (Alle) }}(\infty)$ & $\begin{array}{l}149 \\
(-52)\end{array}$ & $\begin{array}{l}0,0 \\
(0,0)\end{array}$ & $\begin{array}{l}111 \\
(-28)\end{array}$ & $\begin{array}{l}85 \\
(40)\end{array}$ & $\begin{array}{l}92,0 \\
(8,5)\end{array}$ & $\begin{array}{l}0,0 \\
(0,0)\end{array}$ & $\begin{array}{l}0,0 \\
(0,0)\end{array}$ & $\begin{array}{l}-134 \\
(19)\end{array}$ & $\begin{array}{l}497 \\
(-49)\end{array}$ & $\begin{array}{l}-70 \\
(47)\end{array}$ & $\begin{array}{l}0,0 \\
(0,0)\end{array}$ & $0,91 * * *$ \\
\hline $\mathrm{M}_{\mathrm{SO} 4 \text { (Diff) }} 500 \mathrm{~d}$ & $\begin{array}{l}125 \\
(-46)\end{array}$ & $\begin{array}{l}97 \\
(0,0)\end{array}$ & $\begin{array}{l}102 \\
(-5,0)\end{array}$ & $\begin{array}{l}97,0 \\
(8,7)\end{array}$ & $\begin{array}{l}87 \\
(27)\end{array}$ & $\begin{array}{l}0,0 \\
(0,0)\end{array}$ & $\begin{array}{l}0,0 \\
(0,0)\end{array}$ & $\begin{array}{l}134,0 \\
(-4,26)\end{array}$ & $\begin{array}{l}99,0 \\
(0,39)\end{array}$ & $\begin{array}{l}98,0 \\
(1,96)\end{array}$ & $\begin{array}{l}0,0 \\
(0,0)\end{array}$ & $0,98 * * *$ \\
\hline $\mathrm{M}_{\mathrm{SO} 4 \text { (Diff) }}(\infty)$ & $\begin{array}{l}157 \\
(-60)\end{array}$ & $\begin{array}{l}99 \\
(0,0)\end{array}$ & $\begin{array}{l}96 \\
(11)\end{array}$ & $\begin{array}{l}99,0 \\
(2,6)\end{array}$ & $\begin{array}{l}76 \\
(26)\end{array}$ & $\begin{array}{l}0,0 \\
(0,0)\end{array}$ & $\begin{array}{l}0,0 \\
(0,0)\end{array}$ & $\begin{array}{l}302 \\
(-16)\end{array}$ & $\begin{array}{l}186 \\
(-11)\end{array}$ & $\begin{array}{l}90,0 \\
(2,66)\end{array}$ & $\begin{array}{l}0,0 \\
(0,0)\end{array}$ & $0,95^{* * *}$ \\
\hline $\mathrm{NI}_{\text {SO4(Alle) }} 500 \mathrm{~d}$ & $\begin{array}{l}76 \\
(0,09)\end{array}$ & $\begin{array}{l}0,0 \\
(0,0)\end{array}$ & $\begin{array}{l}278 \\
(-0,18)\end{array}$ & $\begin{array}{l}78,0 \\
(0,10)\end{array}$ & $\begin{array}{l}80,0 \\
(0,04)\end{array}$ & $\begin{array}{l}0,0 \\
(0,0)\end{array}$ & $\begin{array}{l}0,0 \\
(0,0)\end{array}$ & $\begin{array}{l}197,0 \\
(-0,09)\end{array}$ & $\begin{array}{l}108,0 \\
(-0,02)\end{array}$ & $\begin{array}{l}79,0 \\
(0,05)\end{array}$ & $\begin{array}{l}0,0 \\
(0,0)\end{array}$ & $0,76^{* * *}$ \\
\hline $\mathrm{VI}_{\mathrm{SO} 4 \text { (Alle) }} 500 \mathrm{~d}$ & $\begin{array}{c}96,1 \\
(0,0)\end{array}$ & $\begin{array}{l}0,0 \\
(0,0)\end{array}$ & $\begin{array}{l}120 \\
(-0,01)\end{array}$ & $\begin{array}{l}73,6 \\
(0,0)\end{array}$ & $\begin{array}{l}141,0 \\
(-0,02)\end{array}$ & $\begin{array}{l}0,0 \\
(0,0)\end{array}$ & $\begin{array}{l}0,0 \\
(0,0)\end{array}$ & $\begin{array}{l}211,0 \\
(-0,05)\end{array}$ & $\begin{array}{l}189,0 \\
(-0,01)\end{array}$ & $\begin{array}{l}64,0 \\
(0,02)\end{array}$ & $\begin{array}{l}0,0 \\
(0,0)\end{array}$ & $0,67 * * *$ \\
\hline $\mathrm{VI}_{\text {SO4(Alle) }}(\infty)$ & $\begin{array}{l}98 \\
(0,01)\end{array}$ & $\begin{array}{l}0,0 \\
(0,0)\end{array}$ & $\begin{array}{l}114 \\
(-0,04)\end{array}$ & $\begin{array}{l}79,0 \\
(0,13)\end{array}$ & $\begin{array}{l}116,0 \\
(-0,03)\end{array}$ & $\begin{array}{l}0,0 \\
(0,0)\end{array}$ & $\begin{array}{l}0,0 \\
(0,0)\end{array}$ & $\begin{array}{l}130,0 \\
(-0,11)\end{array}$ & $\begin{array}{l}133,0 \\
(-0,04)\end{array}$ & $\begin{array}{l}98,21 \\
(0,0)\end{array}$ & $\begin{array}{l}0,0 \\
(0,0)\end{array}$ & $0,91 * * *$ \\
\hline $\mathrm{Nl}_{\mathrm{SO} 4(\mathrm{Diff})} 500 \mathrm{~d}$ & $\begin{array}{l}49 \\
(0,18)\end{array}$ & $\begin{array}{l}0,0 \\
(0,0)\end{array}$ & $\begin{array}{l}229 \\
(-0,13)\end{array}$ & $\begin{array}{l}82,0 \\
(0,08)\end{array}$ & $\begin{array}{l}139,0 \\
(-0,07)\end{array}$ & $\begin{array}{l}0,0 \\
(0,0)\end{array}$ & $\begin{array}{l}0,0 \\
(0,0)\end{array}$ & $\begin{array}{l}39,0 \\
(0,06)\end{array}$ & $\begin{array}{l}80,0 \\
(0,05)\end{array}$ & $\begin{array}{l}21,48 \\
(0,17)\end{array}$ & $\begin{array}{l}0,0 \\
(0,0)\end{array}$ & $0,67^{* *}$ \\
\hline $\mathrm{VI}_{\mathrm{SO} 4(\mathrm{Diff})} 500 \mathrm{~d}$ & $\begin{array}{l}98 \\
(0,0)\end{array}$ & $\begin{array}{l}0,0 \\
(0,0)\end{array}$ & $\begin{array}{l}122 \\
(-0,01)\end{array}$ & $\begin{array}{l}76,0 \\
(0,0)\end{array}$ & $\begin{array}{l}142 \\
(0,02)\end{array}$ & $\begin{array}{l}0,0 \\
(0,0)\end{array}$ & $\begin{array}{l}0,0 \\
(0,0)\end{array}$ & $\begin{array}{l}137,0 \\
(-0,02)\end{array}$ & $\begin{array}{l}92 \\
(0,00)\end{array}$ & $\begin{array}{l}96,0 \\
(0,00)\end{array}$ & $\begin{array}{l}0,0 \\
(0,0))\end{array}$ & $0,94 * * *$ \\
\hline $\mathrm{VI}_{\mathrm{SO} 4 \text { (Diff) }}(\infty)$ & $\begin{array}{l}93 \\
(0,02)\end{array}$ & $\begin{array}{l}0,0 \\
(0,0)\end{array}$ & $\begin{array}{l}101 \\
(0,0)\end{array}$ & $\begin{array}{l}46,0 \\
(0,33)\end{array}$ & $\begin{array}{l}138,0 \\
(-0,08)\end{array}$ & $\begin{array}{l}0,0 \\
(0,0)\end{array}$ & $\begin{array}{l}0,0 \\
(0,0)\end{array}$ & $\begin{array}{l}117,0 \\
(-0,06)\end{array}$ & $\begin{array}{l}110,0 \\
(-0,01)\end{array}$ & $\begin{array}{l}79,0 \\
(0,04)\end{array}$ & $\begin{array}{l}0,0 \\
(0,0)\end{array}$ & $0,70 * * *$ \\
\hline
\end{tabular}

\begin{tabular}{|c|c|c|}
\hline $\begin{array}{l}\mathrm{R} \\
\mathrm{M} \\
\mathrm{NO}_{3} \\
\mathrm{SO}_{4} \\
500 \mathrm{~d} \\
(\infty) \\
\text { Alle }\end{array}$ & $\begin{array}{l}=\text { Denitrifikationsrate zu Versuchsbeginn in } \mathrm{mg} \mathrm{N}^{-1} \mathrm{~kg}^{-1} \mathrm{~d} \\
=\text { Kumulierte Menge in mg N/kg bzw. } \mathrm{mg} \mathrm{S}^{-1} \mathrm{~kg}^{-1} \mathrm{~d} \\
=\text { Nitratreduktion } \\
=\text { Sulfatproduktion } \\
=\text { Bezogen auf Inkubationszeit von } \mathrm{T}_{0} \text { bis } \mathrm{T}_{500} \\
=\text { Bezogen auf unendliche Inkubationszeit } \\
=\text { "Alle Proben" }(\mathrm{N}=233)\end{array}$ & 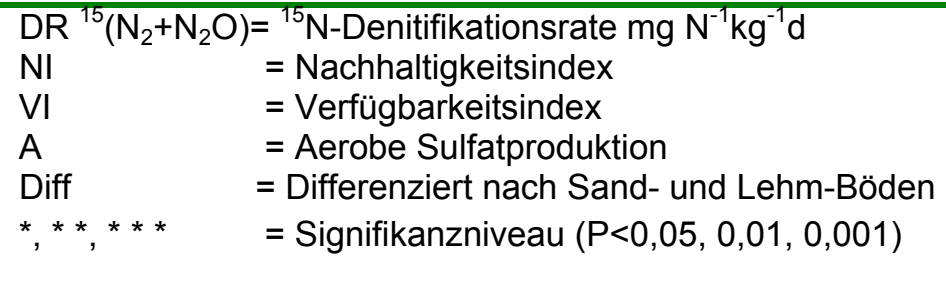 \\
\hline
\end{tabular}



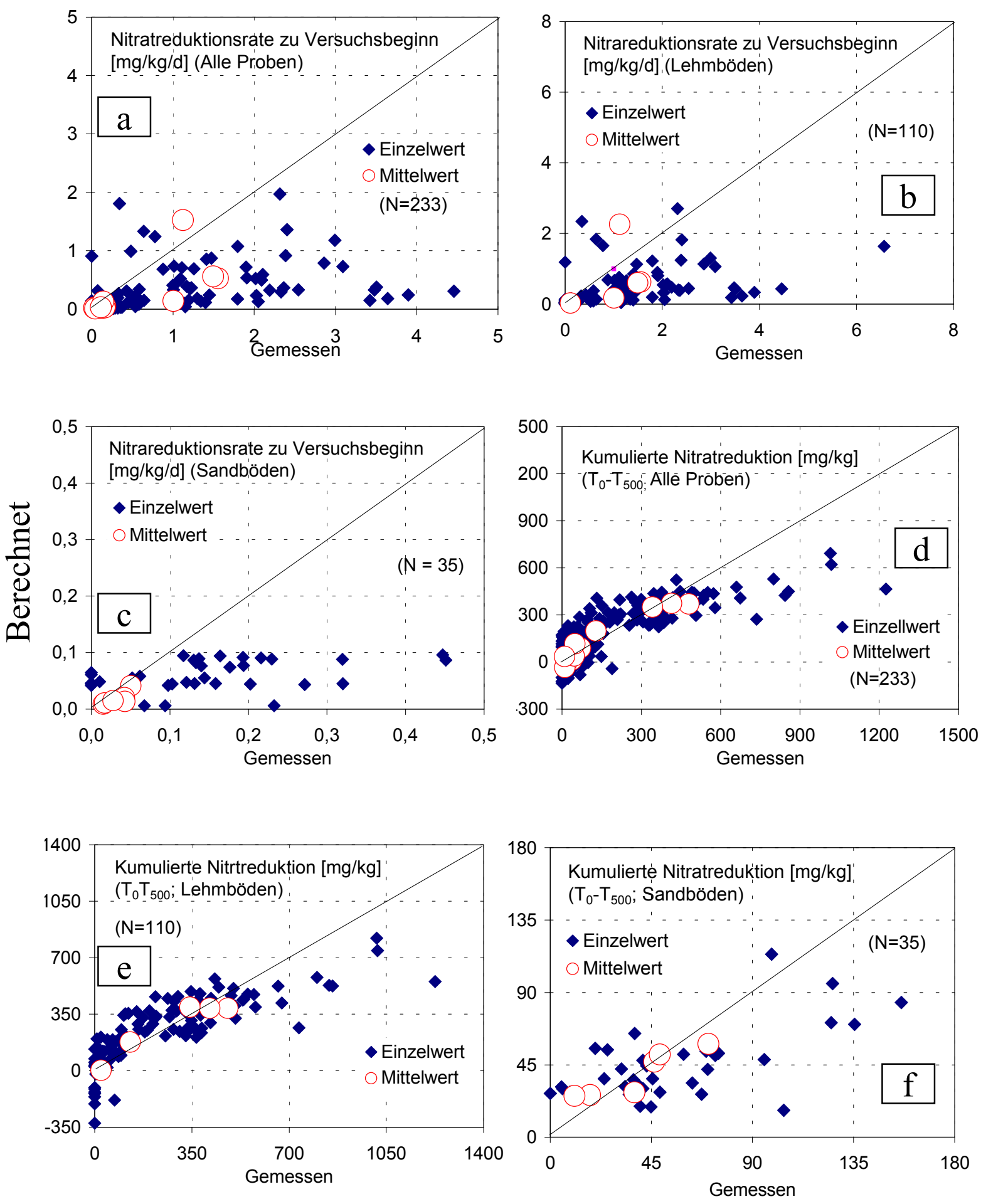

Abb. 8: Vergleich zwischen gemessenen und berechneten Werten für die Variablen Nitratreduktionsrate zu Versuchsbeginn $\left(\mathrm{R}_{\mathrm{NO} 3} ; \mathrm{a}-\mathrm{c}\right)$ und kumulierte Nitratreduktion $\left(\mathrm{M}_{\mathrm{NO} 3} ; \mathbf{d}-\mathrm{f}\right)$ 
Bei $\mathrm{M}_{\mathrm{NO} 3}(500 \mathrm{~d}$, Diff) tritt nur bei einer Fläche (Bühren) eine Abweichung auf, die den Faktor 2 überschreitet (d. h. $>50 \%$ oder $<200 \%$ ). Wegen der geringen Aktivität dieser Fläche ist die absolute Differenz mit $22 \mathrm{mg} \mathrm{N} \mathrm{kg}^{-1}$ jedoch nicht größer als im Mittel der übrigen Flächen ( 7 - $91 \mathrm{mg} \mathrm{N} \mathrm{kg}^{-1}$ ). Die übrigen berechneten Flächenmittelwerte liegen zwischen 74 und $194 \%$ der Messwerte. Bei $\mathrm{M}_{\mathrm{SO} 4}(500 \mathrm{~d}$, Diff) ist die Übereinstimmung noch besser, die berechneten Werte betragen zwischen 87 und $134 \%$ der Messwerte.

Bemerkenswert ist die gute Vorhersage der Sulfidverfügbarkeit $\left(\mathrm{VI}_{\mathrm{SO}} 4 \mathrm{Diff}, \mathrm{R}^{2}=0,94\right)$.

Die Nitratreduktionsrate zu Versuchsbeginn ist nur mäßig vorhersagbar $\left(R^{2}=0,36-\right.$ $0,45)$. Gleiches gilt für den Verfügbarkeitsindex der Nitratreduktion $\left(\mathrm{VI}_{\mathrm{NO}} ; \mathrm{R}^{2}=0,15-\right.$ 0,46). Beim Nachhaltigkeitsindex der Nitratreduktion $\left(\mathrm{NI}_{\mathrm{NO} 3}\right)$ ist die Korrelation zwischen berechneten und gemessenen Werten nicht signifikant, diese Größe ist also nicht vorhersagbar.

Zusammenfassend lässt sich folgendes feststellen:

- Zuverlässige Prognosen für einzelne Proben sind bei keinem Parameter zu erzielen.

- Die Qualität der Prognosen für Flächenmittelwerte ist für die einzelnen Parameter sehr unterschiedlich.

- Gute Übereinstimmungen zwischen gemessenen und berechneten Flächenmittelwerten werden für $\mathrm{M}_{\mathrm{NO} 3(\mathrm{Alle})} 500 \mathrm{~d}$ ); $\mathrm{M}_{\mathrm{NO} 3 \text { (Diff) }} 500 \mathrm{~d}$ ), $\mathrm{M}_{\mathrm{SO} 4(\mathrm{Alle})} 500 \mathrm{~d}, \mathrm{M}_{\mathrm{SO} 4(\mathrm{Alle})}(\infty)$; $\mathrm{M}_{\mathrm{SO} 4(\text { Diff) }} 500 \mathrm{~d}, \mathrm{M}_{\mathrm{SO} 4(\text { Diff) }}(\infty), \mathrm{VI}_{\mathrm{SO} 4(\text { Alle) }}(\infty)$; $\mathrm{VI}_{\mathrm{SO} 4 \text { (Diff) }} 500 \mathrm{~d}$ und $\mathrm{VI}_{\mathrm{SO} 4(\text { Diff) }}(\infty)$ ) erzielt.

- Befriedigende Übereinstimmungen zwischen gemessenen und berechneten Flächenmittelwerten werden für $\mathrm{R}_{\mathrm{NO} 3 \text { (Alle), }} \mathrm{R}_{\mathrm{NO} 3 \text { (Diff); }} \mathrm{M}_{\mathrm{NO} 3 \text { (Alle) }}(\infty) ; \quad \mathrm{M}_{\mathrm{NO} 3 \text { (Diff) }}(\infty)$ $\mathrm{VI}_{\mathrm{NO} 3(\mathrm{Alle})}(\infty), \quad \mathrm{VI}_{\mathrm{NO} 3 \text { (Diff) }} 500 \mathrm{~d}, \quad \mathrm{VI}_{\mathrm{NO} 3 \text { (Diff) }}(\infty) ; \quad \mathrm{VI}_{\mathrm{SO} 4 \text { (Alle) }} 500 \mathrm{~d}, \quad \mathrm{NI}_{\mathrm{SO} 4 \text { (Diff) }} 500 \mathrm{~d}$, $\mathrm{VI}_{\mathrm{SO} 4(\text { Diff) }}(\infty)$ erzielt.

- Die Nachhaltigkeit der Nitratreduktion $\left(\mathrm{NI}_{\mathrm{NO} \text { (Alle) }} 500 \mathrm{~d}, \mathrm{NI}_{\mathrm{NO} \text { (Diff) }} 500 \mathrm{~d}\right.$ ) lässt sich nicht vorhersagen.

- Durch die Differenzierung zwischen Lehm und Sandböden ließen sich die Prognosen erheblich verbessern. 


\section{Diskussion}

\subsection{Variabilität der unabhängigen Parameter und ihre Bedeutung für die Deni- trifikation}

\subsubsection{Gesamt-Vorräte an $\mathbf{C}_{\text {org }}$ und Sulfid}

Im Hinblick auf die Charakterisierung der Standorte nach den Vorräten an $\mathrm{C}_{\text {org }}$ und Sulfid lässt sich anhand der zuvor dargestellten Ergebnisse folgendes festhalten:

Die Gehalte an $\mathrm{C}_{\text {org }}$ und Sulfid schwanken insgesamt in weiten Grenzen. In den Sand-

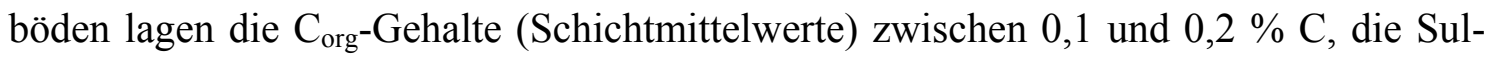
fidgehalte zwischen $0,5-120 \mathrm{mg} \mathrm{S} \mathrm{kg}^{-1}$ (vgl. Tab. 5 und 6). In den Lehmböden waren die Gehalte insgesamt höher $\left(\mathrm{C}_{\text {org }}=0,1-2,8 \%\right.$ und Sulfid $\left.=9,5-7905 \mathrm{mg} \mathrm{S} \mathrm{kg}^{-1}\right)$. Es ist anzunehmen, dass die Unterschiede im Niveau der $\mathrm{C}_{\text {org }}$-Gehalte zwischen Sand- und Lehmböden durch die Entstehungsgeschichte der Sedimente zu erklären ist: Hohe Gehalte treten in den $\mathrm{C}_{\text {org }}$-reichen Auen- und Marschsedimenten des Holozäns auf, geringe

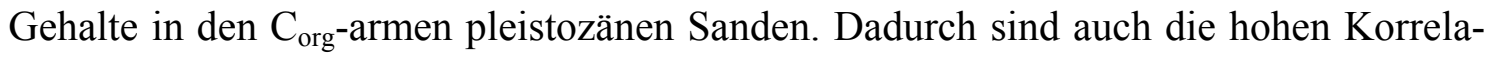
tionen zwischen Texturparametern und $\mathrm{C}_{\text {org }}$ (Tab. 9 a) zu erklären.

Das Vorkommen von partikulärer organischer Substanz und reduzierten SchwefelEisen-Verbindungen im Untergrund und deren Bedeutung als Reduktionsmittel für die Denitrifikation wurde von verschiedenen Autoren berichtet (KöLlE et al., 1985; Rolland, 1995; Lind \& Pedersen, 1976). Die Bedeutung der Denitrifikation im Untergrund wird in erster Linie an den Substraten festgemacht, die für die Denitrifikation verfügbar sind. Zusätzlich stellt sich jedoch die Frage nach der Herkunft der organischen Substanzen im Untergrund. Bei dem organischen Kohlenstoff im Unterboden kann es sich grundsätzlich sowohl um rezente Einträge (Wurzeln, Eintrag gelöster organischer Substanz mit dem Sickerwasser) als auch um fossiles Material (Braunkohle, Torf, Oberbodenmaterial) handeln, welches mit dem Sediment abgelagert wurde (HoffMAnN, 1991). Dabei ist von Bedeutung, dass ein Abbau von fossilem Material irreversibel ist. Bei den untersuchten Böden sind verschiedene Herkünfte anzunehmen: Es ist davon auszugehen dass es sich im Untergrund der Auenböden um fossilen Kohlenstoff von überdeckten oder erodierten Oberböden handelt und dass die Marschböden

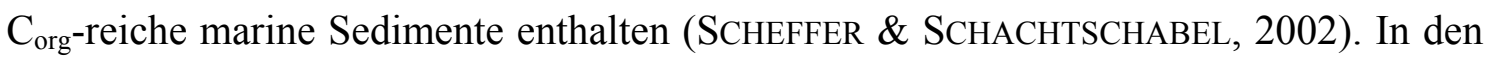
Sandböden können folgende C-Herkünfte eine Rolle spielen: fossile Beimengen 
(Braunkohle, Torf) aus der Ablagerung der Sande und Eintrag von Kohlenstoff aus dem Oberboden der im Zuge der Podsolierung gelöst wurde (HoFFMANN, 1991). Einige Böden enthielten auch eindeutig unregelmäßig verteilt braunkohleartige Partikel oder fossile Wurzeln, was im Probenmaterial visuell erkennbar war.

GERIES (1989) stellt durch eine Hochrechnung plausibel fest, dass fossile organische Kohlenstoff-Vorräte in der Dränzone von Lössböden keine umfangreiche Quelle für die mikrobielle Verwertung darstellen. Aus Untersuchungen der Konzentration des Bodenwassers an $\mathrm{C}_{\text {lös }}$ und der Gesamtmenge an $\mathrm{C}_{\text {org }}$ im Untergrund von Löss-Böden auf der Donau-Hochterrasse ergab sich auch kein Hinweis auf eine erhöhte rezente C-Nachlieferung aus dem Vorrat des Oberbodens (FUNK, 1993). Dies stimmt mit den Befunden aus der ungesättigten Zone der untersuchten Böden überein, die im Vergleich zur gesättigten Zone auf allen Flächen durchweg geringe Gehalte an $\mathrm{C}_{\text {org }}$ aufwies.

Die Sulfidgehalte variieren analog wie die $\mathrm{C}_{\text {org }}$-Gehalte in einem großen Schwankungsbereich. Dabei zeigte sich eine kontinuierliche Zunahme mit zunehmender Tiefe. Da nach FISCHER (1989) in vielen Böden der größte Teil des Gesamt-Schwefels gekoppelt an die organischen Substanz vorkommt, liegt die Vermutung nahe, dass der zunehmende Sulfidgehalt mit dem zunehmenden organischen Kohlenstoffgehalt zusammenhängt. Dies ist aus der engen Korrelation zwischen $\mathrm{C}_{\text {org }}$ und Sulfid eindeutig ersichtlich (Tab. 9 a).

Nach FISCHER (1989) beträgt der Gesamt-S-Gehalt in Böden maximal nur einige Prozent. Der höchste Messwert in einem der untersuchten Lehmböden betrug $0,96 \%$ und lag damit in der Größenordnung der in Böden maximal zu erwartenden Gehalte.

\subsubsection{Vorräte an löslichem Kohlenstoff}

Die Vorräte an heißwasserlöslichem Kohlenstoff $\left(\mathrm{C}_{\mathrm{hwl}}\right)$ liegen zwischen 305 und $2900 \mathrm{mg} \mathrm{C} \mathrm{kg}^{-1}$ (Lehmböden) bzw. zwischen 109 und $484 \mathrm{mg} \mathrm{C} \mathrm{kg}^{-1}$ (Sandböden) (Tab. 5 und 6). Die Streubreite der Gehalte an gelöstem organischem Kohlenstoff $\left(\mathrm{C}_{\text {lös }}\right)$ ist für Sand- und Lehmböden etwa gleich und beträgt $8-40 \mathrm{mg} \mathrm{C}$ $\mathrm{kg}^{-1}$. Diese beiden C-Fraktionen werden in der Literatur hinsichtlich ihrer mikrobiellen Verfügbarkeit unterschiedlich bewertet. Nach SoucI (1938) werden 
durch die Extraktion mit heißem Wasser nicht nur leicht verfügbare Kohlenstoffe, sondern auch Huminstoffe erfasst. Dass $\mathrm{C}_{\mathrm{hwl}}$ nicht vollständig mikrobiell verfügbar ist, wird durch die vorliegenden Untersuchungen bestätigt: Bei den Sand-Böden liegen die $\mathrm{C}_{\mathrm{hwl}}$-Vorräte $\left(109-484 \mathrm{mg} \mathrm{C} \mathrm{kg}^{-1}\right)$ deutlich höher als der berechnete C-Abbau durch heterotrophe Denitrifikation in Höhe von 10 - 47 mg $\mathrm{C} \mathrm{kg}^{-1}$. Dieser Wert berechnet sich aus der Nitratreduktion durch heterotrophe Denitrifikation $\left(\mathrm{M}_{\mathrm{NO} 3} 500 \mathrm{dH}\right)$ in Höhe von $11-51 \mathrm{mg} \mathrm{N} \mathrm{kg}^{-1}$ unter Verwendung des Verhältnisses von $\mathrm{CO}_{2}-\mathrm{C}: \mathrm{NO}_{3}-\mathrm{N}$ bei der heterotrophen Denitrifikation (vgl. G1., Kap. 2.1; $\left.\mathrm{CO}_{2}-\mathrm{C}: \mathrm{NO}_{3}-\mathrm{N}=0,93: 1\right)$. BEAUCHAMP et al. (1980) stellen in Ober- und Unterböden die engsten Zusammenhänge zwischen dem Gesamtgehalt an organischem Kohlenstoff und der Denitrifikationsrate fest, während BURFORD \& BREMNER (1975) zu der Schlussfolgerung kommen, dass der wasserlösliche Kohlenstoff im Oberboden die verfügbare Fraktion besser beschreibt. Die extrahierbare Kohlenstoffmenge ist nach HofFMANN (1991) im Oberboden größer als im Unterboden. Diese Aussage passt mit den geringen $\mathrm{C}_{\text {lös- }}$ Messwerten der untersuchten Böden zusammen.

Nach MCCARTY \& BREMNER (1992) können umfangreichere Verlagerungsprozesse von $\mathrm{C}_{\text {lös }}$ in der Feinstruktur des Bodens kaum möglich sein, da lösliche organische Verbindungen einem raschen Abbau im Oberboden unterliegen und zusätzlich eine Adsorption an Ton- und Schluffteilchen des Bodens stattfinden kann. Dagegen kann wasserlösliche organische Substanz durchaus über Bypassflüsse entlang von Makroporen in tiefere Bodenschichten gelangen (FUNK, 1993).

VON BEÖCZY \& SCHÄFER (1989) fanden in der ungesättigten Zone unterhalb der Wurzelzone zwischen den teilweise stark streuenden Gehalten an löslichem Kohlenstoff und der Nitratkonzentration keinen Zusammenhang. Sie nahmen an, dass die durch chemische Extraktionen gewonnene C-Fraktion kein geeignetes Maß für die C-Verfügbarkeit für die Denitrifikation darstellt. Auch BEAUCHAMP et al. (1980) haben gezeigt, dass die Bestimmung von löslichem Kohlenstoff nicht geeignet ist, um den verfügbaren Kohlenstoff im Unterboden abzuschätzen. SiEMENS et al. (2003) konnten in Inkubationsversuchen mit Bodensickerwässern direkt nachweisen, dass $C_{\text {lös }}$ im Sicker- und Grundwasser keinen bedeutsamen Beitrag zur Denitrifikation liefert. Die Ergebnisse der untersuchten Böden weisen in die gleiche Richtung: die Korrelationen zwischen $\mathrm{C}_{\text {lös }}$ und den 
Kenngrößen der Denitrifikation $\left(\mathrm{R}_{\mathrm{NO} 3}, \mathrm{M}_{\mathrm{NO} 3}\right)$ waren durchweg vergleichsweise gering (vgl. Tab. 9 a und b)

\subsubsection{C-Verfügbarkeit}

Nach SchUlTe-Kelinghaus (1988) ist eine Ermittlung der Beziehung zwischen der Qualität der organischen Substanz und der Verfügbarkeit für Denitrifikanten unter den jeweiligen Standortbedingungen eine sinnvolle Abschätzung einer Denitrifikationskapazität. Über die Qualität und die Verfügbarkeit des organischen Kohlenstoffs im oberflächennahen Grundwasser kann aufgrund mangelnder Publikationen jedoch keine konkrete Aussage gemacht werden. Für Oberböden gibt es hingegen zahlreiche Publikation zur C-Verfügbarkeit. Die CVerfügbarkeit landwirtschaftlich genutzter Mineralböden weist eine ausgeprägte Beziehung zur Profiltiefe auf (HoffmanN, 1991). Im Ap-Horizont ist sie meist signifikant höher als im Unterboden. Für den Ap-Horizont $(0-20 \mathrm{~cm})$ eines Tonbodens wiesen COLBOURN et al. (1984) eine 6-fach höhere potentielle Denitrifikationsrate als für 20-40 cm bzw. eine 15fach höhere als für 40-60 cm Bodentiefe auf. Nach HofFMANN (1991) ist im Untergrund vorhandene organische Substanz für die Denitrifikanten schwerer bzw. nicht abbaubar gegenüber der organischen Substanz im Oberboden.

Es stellt sich die Frage, inwieweit der adsorbierte bzw. ausgefällte organische Kohlenstoff an den Umsatzprozessen im oberflächennahen Grundwasser beteiligt ist. Aus den Ergebnissen der untersuchten Böden ist ersichtlich, dass die langfristige Verfügbarkeit des $\mathrm{C}_{\mathrm{org}}$-Vorrats für die Denitrifikation $\left(\mathrm{VI}_{\mathrm{NO}_{3}} 500 \mathrm{~d}\right)$ mit 1 - $5 \%$ bzw. 1 - $11 \%\left(\mathrm{VI}_{\mathrm{NO} 3}(\infty)\right)$ (vgl. Tab. 8, Zusammenfassung Flächenmittelwerte) insgesamt recht gering ist. Bemerkenswert ist auch, dass innerhalb des untersuchten Datensatzes keine Beziehung zu irgendeinem der untersuchten Parameter abgesichert werden konnte (vgl. Tab. 9a). Folglich ist zunächst anzunehmen, dass es sich bei der $\mathrm{C}_{\text {org }}$-Verfügbarkeit um eine unspezifische Größe handelt. Die Brutversuche von RoLland (1995) haben ebenso gezeigt, dass nur ein geringer Anteil des C-Gehaltes im Untergrund für die Denitrifikation verfügbar ist. Bei den von ihm durchgeführten Simulationen von Säulenversuchen ergab sich ein verfügbarer Anteil von $5 \%$ des organischen C-Gehaltes. Die vor- 
gestellten Untersuchungsergebnisse stimmen somit gut mit den Angaben in der Literatur überein.

Durch das C/N-Verhältnis ließ sich die C-Verfügbarkeit nicht beschreiben: Zwar waren Unterschiede im $\mathrm{C} / \mathrm{N}-$ Verhältnis zwischen den nach der Textur unterschiedenen Bodengruppen deutlich. $\mathrm{C} / \mathrm{N}$ lag in Lehmböden zwischen 7,5 und 19,8, in den Sandböden zwischen 19 und 42 (vgl. Tab. 5, 6). Die Korrelationen zwischen $\mathrm{C} / \mathrm{N}$ und den Kenngrößen der Denitrifikation $\left(\mathrm{R}_{\mathrm{NO} 3}, \mathrm{M}_{\mathrm{NO} 3}\right)$ waren jedoch nicht signifikant (vgl. Tab. 9b).

\subsubsection{Gehalte an $\mathrm{Fe}^{2+}$}

Unter Berücksichtigung der Gehalte an $\mathrm{Fe}_{\text {lös, }}$, die mit Ausnahme des Standorts Dinklage $\left(0,9-38,6 \mathrm{mg} \mathrm{kg}^{-1}\right)$ durchweg gering waren $\left(0,1-7,2 \mathrm{mg} \mathrm{kg}^{-1}\right)$ wird angenommen, dass diese in den untersuchten Böden für den Abbau von Nitrat keine bedeutende Rolle spielen. Da die Eisenreduktion gemäß der Abfolge von Redoxreaktionen thermodynamisch gesehen erst nach der Nitratreduktion stattfindet, kommen in der ungesättigten und gesättigten Zone Nitrat und $\mathrm{Fe}^{2+}$ theoretisch nicht gleichzeitig vor (MUNCH \& OTTOW, 1983; KöLLE, 1987). In folgenden Situationen ist aber durchaus eine Abweichung von dieser Regel möglich: Erstens kann Nitrat durch Einwaschung in hydromorphen Böden in stark anaerobe, $\mathrm{Fe}^{2+}$-haltige Kompartimente hinein diffundieren und dann reduziert werden (RICHTER \& RICHTER, 1991). Zweitens kann $\mathrm{Fe}^{2+}$ aus der Oxidation von Pyrit- bzw. Markasit-Schwefel freigesetzt werden, wobei sowohl Nitrat als auch Sauerstoff als Oxidationsmittel verantwortlich sein kann. Drittens kann eine Nachlieferung von $\mathrm{Fe}^{2+}$ vom Austauscher (Tonminerale) in einem reduzierenden, nitratreichen Grundwasser erfolgen (KöLlE, 1988). Ob diese Prozesse in Dinklage eine bedeutende Rolle spielen, kann anhand der Versuchsergebnisse nicht geklärt werden, da keine Informationen zum Beitrag von $\mathrm{Fe}^{2+}$ zum Nitratabbau vorliegen. 


\subsection{5 pH-Wert}

Die untersuchten Böden weisen ein weites Spektrum von $\mathrm{pH}-$ Werten auf $(\mathrm{pH} 3,2$ - 7,3, vgl. Tab. 5 und 6). Im allgemeinen wird nach BuRTH \& OтTOW (1982) keine Abhängigkeit zwischen der Denitrifikation und dem pH-Wert festgestellt. Es werden jedoch unterschiedliche Angaben über die Nitratverluste unter sauren Bedingungen gemacht. So beobachteten COPER \& SMITH (1963) bei Denitrifikationsversuchen mit Böden verschiedener pH-Werte zwischen den Böden innerhalb der einzelnen $\mathrm{pH}$-Gruppen größere Schwankungsbereiche der Aktivität als zwischen den sauren und neutralen Böden. Aus Feld- und Laborversuchen mit aufgekalkten Moor- und Mineralböden (A-Horizont) stellten WARING \& GILLIAM (1983) heraus, dass der denitrifikationsfördernde Effekt einer pH-WertErhöhung in der C-Mineralisierung liegt.

In den untersuchten Böden war die Denitrifikation mit dem $\mathrm{pH}-$ Wert zwar positiv korreliert (vgl. Tab. 9). Diese Beziehung ist jedoch in erster Linie dadurch zu erklären, dass die relativ sauren Sandböden auch geringere $\mathrm{C}_{\text {org-Gehalte aufwie- }}$ sen. Falls ein pH-Einfluss auf die Denitrifikation vorlag, wurde dieser jedenfalls durch den $\mathrm{C}_{\text {org }}$-Effekt völlig überlagert.

\subsection{Denitrifikationsraten, langfristiges Denitrifikationspotential}

Bedeutende Denitrifikationsraten wurden an fast allen Lehmböden (vgl. Tab. 8) festgestellt. $\mathrm{R}_{\mathrm{NO} 3}$ lag zwischen 0,11 und $1,56 \mathrm{mg} \mathrm{kg}^{-1} \mathrm{~d}^{-1}$. Im Gegensatz dazu schwankten die Denitrifikationsraten in den Sandböden zwischen 0 und 0,35 mg $\mathrm{kg}^{-1} \mathrm{~d}^{-1}$ und waren somit vergleichsweise gering. Die Korrelationen der Denitrifikationsrate mit den unabhängigen Parametern (Tab. 9 a, b) sowie die Regressionsanalyse (Tab. 10) belegen eindeutig, dass die Vorräte an reduzierten Verbindungen $\left(\mathrm{C}_{\mathrm{org}}\right.$, Sulfid) die wesentliche Steuergröße für die Intensität der Denitrifikation darstellen. Dieser Zusammenhang ist in der Literatur durch zahlreiche Studien belegt (vgl. die Literaturübersichten bei RoLlAND, 1995; HOFFMANN, 1991; FUNK, 1993 und MCCARTY \& BREMNER, 1993).

TRUDELL et al. (1986) wiesen für einen reduzierenden oberflächennahen Sandaquifer mittels Injektionsversuch bei einem $\mathrm{C}_{\text {org }}$-Gehalt von $0,08-0,13 \% \mathrm{im}$ 
Aquifer eine Denitrifikationsrate von ca. $3 \mathrm{mg} \mathrm{NO}-\mathrm{N}^{-1} \mathrm{~d}^{-1}$ nach. WeLL et al. (2001) stellten bedeutende Denitrifikationsraten im oberflächennahen Grundwasser von hydromorphen Böden fest. Nach einer Differenzierung nach den Bodenarten Sand und Lehm lagen für Lehmböden die Maximal-Werte bei 6,5 mg $\mathrm{NO}_{3}-\mathrm{N} \mathrm{kg}^{-1} \mathrm{~d}^{-1}$ und für Sandböden bei $0,26 \mathrm{mg} \mathrm{NO}-\mathrm{N} \mathrm{kg}^{-1} \mathrm{~d}^{-1}$. In einem humusarmen Unterboden $\left(\mathrm{C}_{\text {org }}\right.$-Gehalt $\left.=0,2 \%\right)$ eines Podsols (aus Sand) stellte RoLLAND (1995) mittels Säulenversuchen mit wassergesättigten Bedingungen eine Abbaurate von $5 \mathrm{mg} \mathrm{NO}-\mathrm{N}^{-1} \mathrm{~d}^{-1}$ fest. Im Unterboden eines Kolluviums

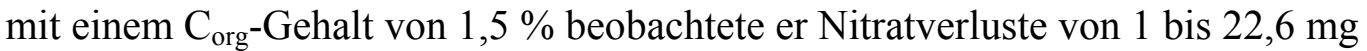
$\mathrm{NO}_{3}-\mathrm{N}^{-1} \mathrm{~d}^{-1}$. Die Abbauraten der untersuchten Böden liegen somit in der gleichen Größenordnung wie die zitierten Literaturangaben.

Die langfristige kumulierte Nitratreduktion $\left(\mathrm{M}_{\mathrm{NO} 3}(\infty)\right)$ betrug in den Lehmböden zwischen 13,3 und $1102 \mathrm{mg} \mathrm{N} \mathrm{kg}^{-1}$, in den Sandböden zwischen 15 und $81 \mathrm{mg} \mathrm{N}$ $\mathrm{kg}^{-1}$ (vgl. Tab. 8). Diese Messgröße wurde ebenfalls durch die Vorräte an Sulfid und $\mathrm{C}_{\text {org }}$ bestimmt (vgl. Tab. 9 a, b und Tab. 10).

ROLLAND (1995) wies mittels Inkubationsversuchen in verschiedenen Oberböden eine Denitrifikation zwischen 140 und $280 \mathrm{mg} \mathrm{N} \mathrm{kg}^{-1}$ und in den Unterböden eines Podsols und eines Gleys zwischen 80 und $100 \mathrm{mg} \mathrm{N} \mathrm{kg}^{-1}$ nach. BÖTTCHER et al. (1985) ermittelten im sulfidhaltigen Sand-Aqifer des Fuhrberger Feldes eine langfristige Nitratreduktion von $318 \mathrm{mg} \mathrm{NO}_{3}-\mathrm{N}^{-1}$. FUNK (1993) registrierte in verschiedenen Zeiträumen in der Dränzone eines Lössstandortes in 2,7-5 m Tiefe eine langfristige Nitratreduktion von insgesamt $655 \mathrm{~kg} \mathrm{NO}_{3}-\mathrm{N}$ $\mathrm{ha}^{-1}$. Diese Werte kommen den eigenen Untersuchungsergebnissen sehr nahe.

\subsection{Bedeutung der autotrophen Denitrifikation, Verfügbarkeit von $C_{\text {org }}$ und Sulfid für die Denitrifikation}

\subsubsection{Autotrophe Denitrifikation}

Unter anaeroben Bedingungen gilt die heterotrophe Denitrifikation als Hauptweg der dissimilatorischen Nitratreduktion. Im Untergrund kann generell neben der heterotrophen Denitrifikation auch die autotrophe Denitrifikation eine bedeutende Rolle spielen, bei der Sulfide oxidiert werden und somit Sulfat als Reaktionsprodukt freigesetzt wird. Dabei dient Kohlendioxid als C-Quelle. Au- 
totrophe Denitrifikation wurde bisher in verschiedenen Grundwassereinzugsgebieten von KöLle et al. (1983), VAN BEEK et al. (1988) und POSTMA et al. (1991) beobachtet. Dieser Prozess kann nach RoLLAND (1995) nicht nur in der gesättigten, sondern auch in der ungesättigten Zone einen Beitrag liefern.

Die parallel zur Nitratreduktion festgestellte Sulfatproduktion in den Tiefenprofilen der sulfidhaltigen Standorte unter anaerober Inkubation liefern sichere Hinweise auf autotrophe Denitrifikationsprozesse im oberflächennahen Grundwasser der untersuchten Böden. Der Vergleich zwischen Sand- und Lehmböden hinsichtlich der Nitratreduktion und Sulfatproduktion in den Inkubationsversuchen lässt folgende Schlüsse zu: Das langfristige Potential der Sulfatbildung $\left(\mathrm{M}_{\mathrm{SO} 4}\right)$ ist ein Maß für den langfristigen Beitrag der autorophen Denitrifikation zum Nitratabbau. In den sulfidhaltigen Böden betrug dieser Wert zwischen 2 und $21 \mathrm{mg} \mathrm{S} \mathrm{kg}^{-1}$ (Sandböden) bzw. zwischen 59 und $225 \mathrm{mg} \mathrm{S} \mathrm{kg}^{-1}$ (Lehmböden) (vgl. Tab. 8). Nach Rolland (1995) beträgt die aus den Pyritgehalten errechenbare maximale Sulfatbildung im Untergrund des Gleys zwischen 936 und $3602 \mathrm{mg} \mathrm{S} \mathrm{kg}{ }^{-1}$. BÖTTCHER (1992) beschreibt Sulfatbildungskapazitäten im Fuhrberger Feld von 900 - $5000 \mathrm{mg} \mathrm{S} \mathrm{kg}^{-1}$.

STREBEL et al. (1985b) ermittelten aus einer Anzahl von Inkubationsversuchen mit unterschiedlichen Aquifermaterialien aus Niedersachsen ein mittleres Sulfatbildungsvermögen der Sedimente zwischen 0,3 und $1,2 \mathrm{~kg} \mathrm{SO}{ }_{4}{ }^{2-} / \mathrm{m}^{-3}$ Sediment. Somit stimmen die vorgestellten Untersuchungsergebnisse in der Größenordnung mit den Angaben aus der Literaturübersicht überein.

\subsubsection{Vergleich von C- und S-Verfügbarkeit}

Aus den Zeitkurven der Nitratreduktion und Sulfatproduktion (vgl. Abb. 5) ist erkennbar, dass eine Nitratreduktion bei den meisten Proben über den gesamten Inkubationszeitraum erfolgte, während die Sulfatproduktion in den meisten Sulfidhaltigen Proben schon vor dem Versuchsende (400 - 700 Tagen nach $\mathrm{T}_{0}$ ) vollständig zum Erliegen kam. ROLLAND (1995) wies bei Inkubationsversuchen mit Proben aus dem Kapillarsaum des Grundwassers nach, dass der Nitratabbau in maximal 50 Tagen abgeschlossen war. Bei Proben aus Oberböden kam die Nitratreduktion innerhalb von 160 Tagen nicht zum Erliegen. Die im Vergleich 
zur Nitratreduktion rasche Abnahme der Sulfatproduktion verdeutlicht, dass der Anteil der autotrophen Denitrifikation bei allen sulfidhaltigen Böden im Verlauf der Inkubation abnahm. Die Verfügbarkeit (500 Tage) der Sulfidvorräte war mit Werten zwischen 5 und $51 \%$ wesentlich höher als die $\mathrm{C}_{\text {org-Verfügbarkeit von } 2}$ bis $5 \%$ (vgl. Tab.8).

Verschiedene Autoren ermittelten aus der Sulfatbildungskapazität von Sulfidhaltigen Böden Werte für die Sulfidverfügbarkeit: RichtER \& RICHTER (1991) beobachteten in einem Wassereinzugsgebiet bei Hannover Nitratabbau und gleichzeitige Sulfatproduktion durch die Aktivität des Thiobacilus denitrificans in der ungesättigten Zone. Sie ermittelten ein Sulfatbildungsvermögen von ca. 7 - $17 \%$ des Gesamtschwefels. RolLAND (1995) konnte im Brutversuch mit Lössböden 5 - $25 \%$ des Gesamtschwefels als Sulfatproduktion feststellen. BÖTTCHER (1992) fand in einem Überstauversuch in Proben aus dem Aquifer des Fuhrberger Feldes dagegen sogar eine langfristige Sulfatbildung von $50 \%$ des Gesamtschwefels. Größenordnung und Schwankungsbereich dieser Literaturwerte für die Sulfidverfügbarkeiten $(5-50 \%)$ stimmt mit den Werten der Sulfid-haltigen Böden ( 5 - $51 \%$ überein. Dabei zeigten die Sandböden geringere (5 - $15 \%)$ und die Lehmböden höhere Sulfidverfügbarkeiten (19-51\%).

\subsubsection{Aerobe Sulfatproduktion}

Bemerkungswert ist, dass die aerobe Sulfidverfügbarkeit $\left(\mathrm{M}_{\mathrm{SO}_{4}} \mathrm{~A}=19-90 \%\right.$, Tab. 8) bei allen Böden höher war als die anaerobe Sulfatproduktion, was besonders für die Sandböden gilt. Dies bestätigt die Ergebnisse von ROLLAND (1995). Er beobachtete sowohl in Brut- als auch in Säulenversuchen unter aeroben und anaeroben Bedingungen, dass die Oxidation reduzierter SVerbindungen unter aeroben Bedingungen sehr viel schneller ablief. Er begründet dies damit, dass die Oxidation des sehr labilen Schwefels durch Sauerstoff gegenüber der Oxidation mit Nitrat viel schneller abläuft. Daraus folgert er, dass die chemolithoautotrophe Denitrifikation nur dann in der ungesättigten Zone eine Rolle spielen kann, wenn diese dauerhaft $\mathrm{O}_{2}$-frei ist. Er erklärt damit die Unterschiede zwischen einem Gleyboden und einer Parabraunerde: Vermutlich 
wegen der höheren Sauerstoff-Diffusion in der Parabraunerde lagen die Schwefel-Gehalte um den Faktor 10-20 niedriger als in dem Gleyboden.

\subsection{Bedeutung der langfristigen Denitrifikationsdynamik für die Grund- wasserqualität}

Es stellt sich die Frage, in welcher Weise sich der Nitrataustrag aus der ungesättigten Zone langfristig auf die Qualität des Grundwassers nach Passage der untersuchten Schichten auswirkt. Die Verminderung der Nitratfracht ist i.d.R. die zentrale Fragestellung bei der Erforschung der Denitrifikation im Grundwasser (vgl. Kap. 1). Die Endlichkeit dieses Prozesses durch den begrenzten Vorrat an Reduktionsmitteln und die damit verbundene Gefahr eines langfristigen Anstiegs der Nitratgehalte ist dabei nur von relativ wenigen Autoren thematisiert worden (vgl. BötTCher et al., 1985; Rolland, 1995). Quantitative Prognosen für diesen Vorgang liegen bisher nur vereinzelt vor. BÖTTCHER et al. (1985) ermittelten auf der Basis umfangreicher mehrjähriger Geländeuntersuchungen im Wassereinzugsgebiet eines Brunnens des Wasserwerks Fuhrberger Feld nördlich von Hannover eine Nitratabnahme von $318 \mathrm{mg} \mathrm{NO}_{3} 1^{-1}$ auf die Hälfte innerhalb von 1 bis 2 Jahren. Die genannten Autoren haben in einer Fallstudien-Rechnung mit Hilfe deterministischer Simulationsmodelle prognostiziert, dass der Vorrat an reduzierter Substanzen in diesem Aquifer bei anhaltendem Nitrateintrag nach ca. 400 Jahren aufgebraucht sein wird (BÖTTCHER et al., 1989).

Im folgenden wird versucht, aus den vorliegenden Versuchsergebnissen eine Prognose für die langfristige Entwicklung der Nitratgehalte im Grundwasser der untersuchten Böden zu treffen. Unter der Voraussetzung, dass die im Labor bestimmte Prozesskinetik auf Freilandbedingung übertragbar ist, lässt sich aus den Labordaten die langfristige Zeitdynamik des Nitratabbaus der Versuchsflächen abschätzen.

Für die Qualität des Grundwassers nach Passage der untersuchten reaktiven Schichten ergeben sich folgende Regelmechanismen: Wenn von großflächiger Verbreitung dieser Schichten in einem Wassereinzugsgebiet ausgegangen wird, dann kann die Prozesskinetik in erster Näherung als eindimensionaler, vertikaler reaktiver Transport durch die reaktiven Schichten betrachtet werden (Abb. 9). 
Zwar überwiegt i.d.R. die horizontale Komponente bei der Fließrichtung des Grundwassers, wie durch den Verlauf der Stromlinien in Abb. 9 angedeutet wird. Für die Abbaukinetik ist jedoch in erster Linie die Verweilzeit des Grundwassers in der reaktiven Schicht entscheidend. Bei einer flächenhaften Verbreitung und konstanter Mächtigkeit der reaktiven Schicht kann die Verweilzeit, die

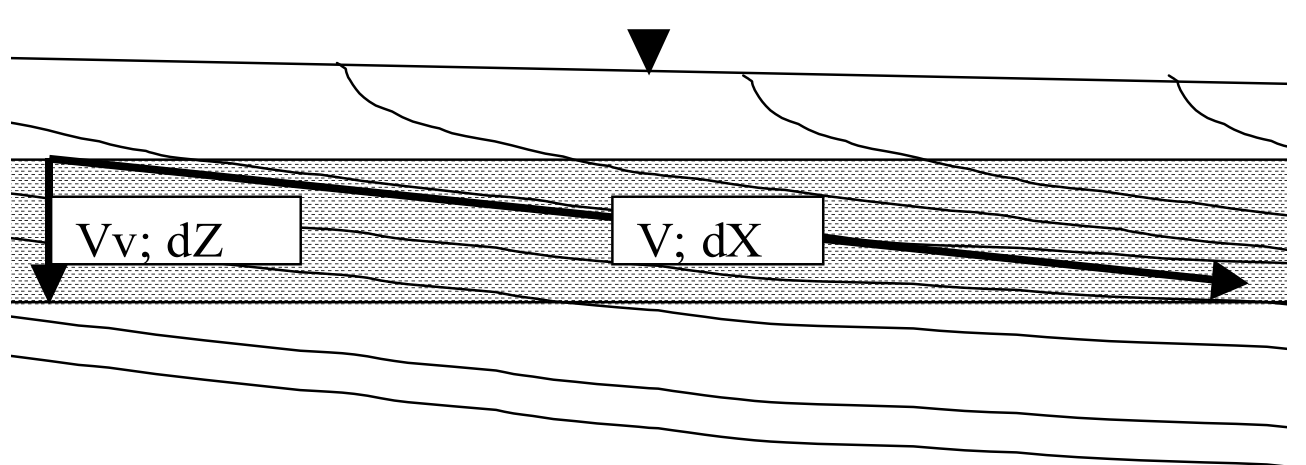

Abb. 9: Vergleich eines realen Fließfeldes im oberflächennahen Grundwasser $(\mathrm{V}=$ Fließgeschwindigkeit, $\mathrm{dX}=$ Fließstrecke) mit der vereinfachten Annahme einer ausschließlich vertikalen Fließrichtung (Vv, $\mathrm{dZ}=$ Vertikalkomponenten der Fließgeschwindigkeit und der Fließstrecke) in einer reaktiven Schicht (gestrichelte Fläche). Die Länge der Pfeile repräsentiert die Beträge der Fließgeschwindigkeiten und der Fließstrecken. Die Verweilzeit in der reaktiven Schicht ergibt sich aus dem Verhältnis von Fließstrecke zur Fließgeschwindigkeit und ist somit für beide Betrachtungsweisen gleich

sich aus der wirklichen, überwiegend horizontalen Fließstrecke und der wirklichen Fließgeschwindigkeit ergibt, auch durch die Sickerwasserrate (S) und die Vertikalkomponente (dZ) der Fließstrecke (dX) ermittelt werden. Somit kann für die untersuchten Standorte auch ohne Kenntnis der wirklichen Grundwasserfließverhältnisse die Verweilzeit abgeschätzt werden.

Die Zeitfunktion der Nitratkonzentration nach Passage der reaktiven Zone im oberflächennahen Grundwasser ist abhängig von der spezifischen Nitratabbaurate der Schicht $\left(\mathrm{R}_{\mathrm{NO} 3}\right)$, von der Schichtmächtigkeit (D), der Porosität (E), der Verweilzeit $\left(\mathrm{T}_{\mathrm{V}}\right)$, der jährlichen Nitratfracht $\left(\mathrm{F}_{\mathrm{NO} 3}\right)$ sowie vom langfristigen Denitrifikationspotential $\left(\mathrm{M}_{\mathrm{NO} 3}\right)$. Für eine exakte Modellierung der Nitratkonzentration im Zeitverlauf wäre es erforderlich, einen konvektiv-dispersiven Transportterm zu verwenden. Für den Nitratabbau müsste eine Kinetik verwendet 
werden, die den Einfluss der Substratkonzentration (Nitrat, Konzentration an verfügbarem $\mathrm{C}_{\text {org }}$ und Sulfid) auf die Denitrifikationsrate berücksichtigt (z.B. Reaktion 1. Ordnung, Michelis-Menten-Kinetik). Eine derartige Modellierung wurde von BÖTTCHER et al. (1989, s.o.) durchgeführt, um die langfristige Nitratkonzentration im Aquifer des Fuhrberger Feldes bei fortschreitender Oxidation des Vorrats an reduzierten Schwefel-Verbindungen vorherzusagen. An dieser Stelle soll nur eine stark vereinfachte Modellierung durchgeführt werden, um die Größenordnung für die zeitliche Dynamik der Nitratkonzentration bzw. der Erschöpfung des Reduktionsmittelvorrats unter variierten Bedingungen aufzuzeigen. Für die Denitrifikation wird eine Reaktion 0-ter Ordnung angenommen, d.h. die Rate des Nitratabbaus wird als unabhängig von den Substratkonzentrationen (Nitratkonzentration im Bodenwasser, Konzentration an verfügbaren Reduktionsmitteln) betrachtet. Es wird also ein konstanter Wert für eine potentielle Denitrifikationsrate $\left(\mathrm{R}_{\mathrm{NO} \text { pot }}\right)$ angenommen, welche so lange gilt, wie der Nitratgehalt größer 0 ist. Dieser Wert wird für die verschiedenen Standorte aus der temperaturkorrigierten mittleren Nitratabbaurate während der Laborinkubation abgleitet:

$\mathrm{R}_{\mathrm{NO} 3 \text { pot, } \mathrm{M}}=\mathrm{M}_{\mathrm{NO} 3}(\infty) /\left(\mathrm{T}_{\text {Abbau, Labor }} \times \mathrm{Q}_{(25 / 10)}\right)$

$\left(\mathrm{M}_{\mathrm{NO} 3}(\infty)=\right.$ kumulierte Nitratreduktion im Laborversuch bei unendlicher Inkubationszeit; $\mathrm{T}_{\text {Abbau, Labor }}=$ Zeit bis zur vollständigen Erschöpfung des Reduktionsmittelvorrats; vgl. Kap. 5.1; $\mathrm{Q}_{(25 / 10)}=$ mittlerer Temperaturquotient für die Nitratreduktion bei 10 und $25^{\circ} \mathrm{C}$, vgl. Kap. 5.4).

Der Nitratabbau erfolgt in der Modellierung in dieser Rate solange der Substratvorrat (Nitrat, Reduktionsmittel) nicht erschöpft ist. Die Restnitratkonzentration des Grundwassers nach Passage der reaktiven Schicht $\left(\mathrm{C}_{\mathrm{NO} 3 \mathrm{r}}\right)$ sowie die Zeit bis zum vollständigen Abbau des Reduktionsmittelvorrats unter Freilandbedingungen $\left(\mathrm{T}_{\text {Abbau }}\right)$ wurde wie folgt ermittelt:

Berechnung der Verweilzeit in der reaktiven Schicht $\left(\mathrm{T}_{\mathrm{V}}\right)$ :

$\mathrm{T}_{\mathrm{V}}=\mathrm{D} \times \mathrm{E} / \mathrm{S}$

Berechnung der potentiellen Nitratabbaumenge $\left(\mathrm{M}_{\mathrm{NO} \text { pot }}\right)$

$\mathrm{M}_{\mathrm{NO} \text { pot }}=\mathrm{R}_{\mathrm{NO} \text { pot, } \mathrm{M}} \times \mathrm{T}_{\mathrm{V}}$

Berechnung der effektiven Nitratabbaumenge $\left(\mathrm{M}_{\mathrm{NO} \text { eff }}\right)$ :

$\mathrm{M}_{\mathrm{NO} \text { eff }}=\mathrm{M}_{\mathrm{NO} 3 \text { pot }} \quad$ wenn $\mathrm{M}_{\mathrm{NO} 3 \text { pot }}<\mathrm{F}_{\mathrm{NO} 3}$ 
$\mathrm{M}_{\mathrm{NO} \text { eff }}=\mathrm{F}_{\mathrm{NO} 3} \quad$ wenn $\mathrm{M}_{\mathrm{NO} \text { pot }}>\mathrm{F}_{\mathrm{NO} 3}$

$\left(\mathrm{F}_{\mathrm{NO} 3}=\right.$ Nitratfracht $)$

Effektive mittlere Nitratabbaurate $\left(\mathrm{R}_{\mathrm{NO} \text { eff }}\right)$

$\mathrm{R}_{\mathrm{NO} \text { eff }}=\mathrm{M}_{\mathrm{NO} \text { eff }} / \mathrm{T}_{\mathrm{V}}$

Zeit bis zum vollständigen Abbau des Reduktionsmittelvorrats $\left(\mathrm{T}_{\mathrm{Abbau}}\right)$

$\mathrm{T}_{\mathrm{Abbau}}=\mathrm{M}_{\mathrm{NO} 3}(\infty) / \mathrm{R}_{\mathrm{NO} 3 \mathrm{eff}}$

Folgende Modellvarianten wurden durchgerechnet: Schichtmächtigkeit (D) $=1$ / $0,5 / 0,1 \mathrm{~m}$; Sickerwasserrate $(\mathrm{S})=120 / 60 / 240 \mathrm{~mm} \mathrm{a}^{-1}$; Nitratfracht $\left(\mathrm{F}_{\mathrm{NO} 3}\right)=$ $100 / 50 / 200 \mathrm{~kg} \mathrm{~N} \mathrm{ha}^{-1} \mathrm{a}^{-1}$.

Abb. 10 zeigt für ein Beispiel (Freistatt, Szenario 1) einen simulierten Zeitgang des Reduktionmittelvorrats. Der Abbau verläuft linear (Reaktion nullter Ordnung) und die Restnitratkonzentration steigt zum Zeitpunkt der Erschöpfung $\left(\mathrm{T}_{\text {Abbau }}\right)$ sprunghaft auf das Niveau der Konzentration im Sickerwasser.

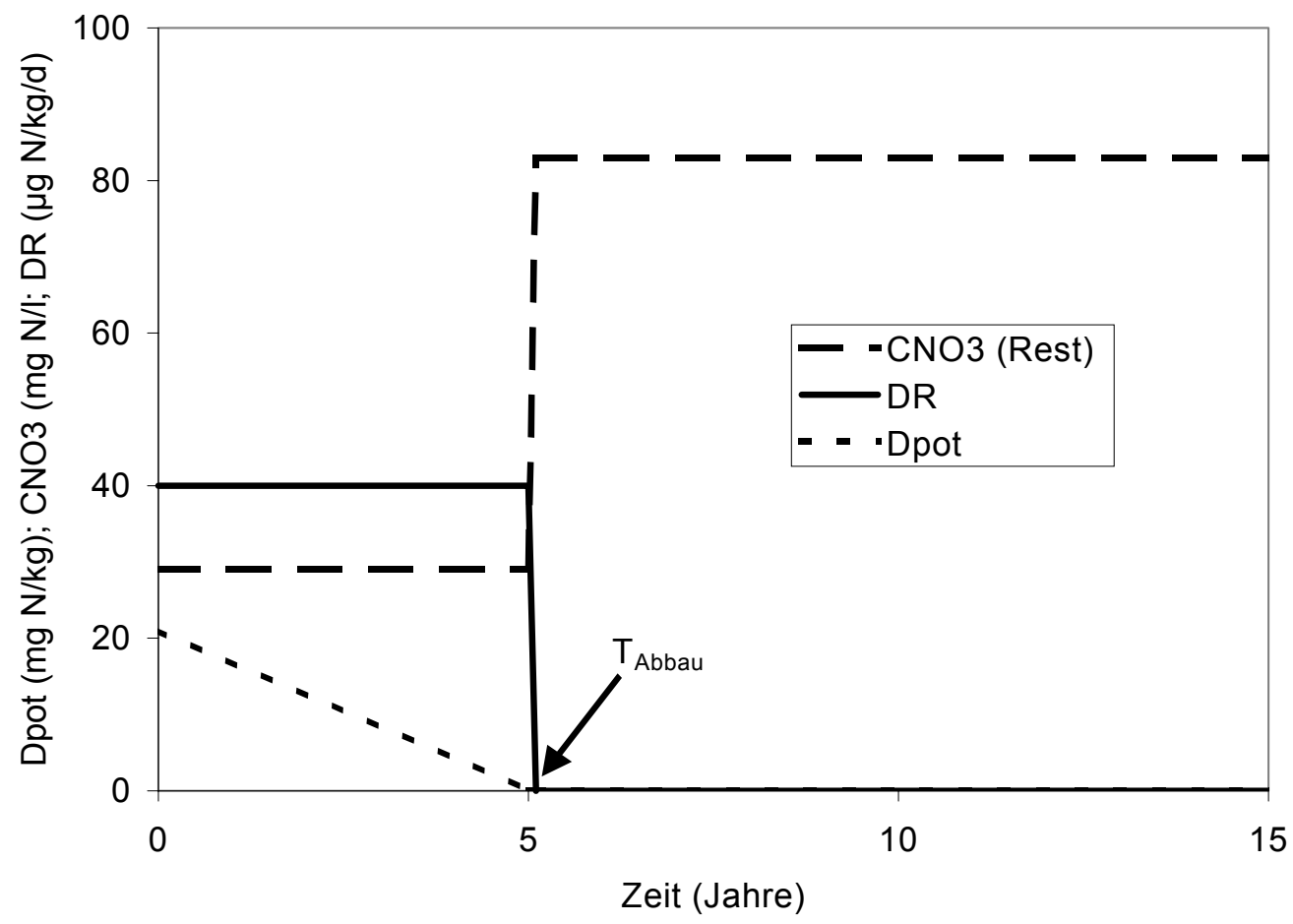

Abb. 10: Simulation der Erschöpfung des langfristigen Denitrifikationspotentials $\left(D_{\text {pot }}\right)$ durch fortschreitenden Abbau des Reduktionsmittelvorrats (Beispiel Freistatt, Szenario 1, vgl. Tab. 12). $\mathrm{C}_{\mathrm{NO} \text {, Rest }}=$ Nitratkonzentration im Grundwasser nach Passage der reaktiven Schicht; $\mathbf{D R}=$ Denitrifikationsrate, $\mathbf{T}_{\text {Abbau }}=$ Zeit bis zum vollständigen Abbau des Reduktionsmittelvorrats 
Wegen der geringen Nitratabbaurate ist die Denitrifkation in der reaktiven Schicht hier unvollständig (Restnitrat $>0$ ). In Tab. 12 sind die Werte für $\mathrm{C}_{\mathrm{NO} 3 \mathrm{r}}$ sowie $\mathrm{T}_{\mathrm{Abbau}}$ für 8 Szenarien aufgetragen und zwar für alle Flächen. $\mathrm{R}_{\mathrm{NO}}$ spielt nur eine Rolle, sofern der Wert so gering ist, dass kein vollständiger Nitratabbau stattfindet $\left(\mathrm{C}_{\mathrm{NO} 3 \mathrm{r}}>0\right.$, z.B. Fuhrberg, Freistatt, Bühren). Bei diesen Standorten steuert die Sickerwasserrate die Höhe von $\mathrm{C}_{\mathrm{NO} \text { rr }}$. Die Sickerwasserrate ist ohne Einfluss auf die Erschöpfungszeit. N-Saldo $\left(\mathrm{F}_{\mathrm{NO} 3}\right)$ und Schichtmächtigkeit (D) steuern die Erschöpfungszeit $\left(\mathrm{T}_{\mathrm{Abbau}}\right)$. Bei Standorten mit unvollständigem Nitratabbau ist der Saldo irrelevant.

Es stellt sich die Frage, inwieweit diese stark vereinfachte Modellierung eine realistische Schätzung für den langfristigen Nitratabbau liefert. Um dies zu prüfen, wurden die Randbedingungen der Modellierung von BöTTCHER et al. (1989, s.o.) verwendet. Die Schichtmächtigkeit des Fuhrberger Aquifers beträgt 20 $30 \mathrm{~m}$. In der Modellierung wurde ein Vorrat an reduzierten Schwefelverbindungen von $200 \mathrm{mg} \mathrm{S} \mathrm{kg}^{-1}$ Sediment angenommen. Bei vollständiger Abbaubarkeit entspricht dies einer potentiellen Nitratreduktion $\left(\mathrm{M}_{\mathrm{NO} 3}\right)$ von $122 \mathrm{mg} \mathrm{N} \mathrm{kg}^{-1}(\mathrm{vgl}$. Stöchiometrie der autotrophen Denitrifikation, Kap. 2.1). Die Nitratkonzentration der Grundwasserneubildung betrug $130 \mathrm{mg} \mathrm{NO}_{3} \mathrm{l}^{-1}$. Aus dieser Konzentration lässt sich mit Hilfe der mittleren Grundwasserneubildung des Fuhrberger Feldes (200 mm a ${ }^{-1}$, DuIJNISVELD et al., 1993) eine Nitratfracht von $64 \mathrm{~kg} \mathrm{~N} \mathrm{ha}^{-1} \mathrm{a}^{-1}$ ableiten. Setzt man diese Randbedingungen in die vereinfachte Simulationsrechnung ein, so ergeben sich Abbauzeiten zwischen 570 Jahren $(20 \mathrm{~m}$ Schichtmächtigkeit) und 850 Jahren (30 m Schichtmächtigkeit). Es ist ersichtlich, dass diese Ergebnisse größenordnungsmäßig mit der komplexen Simulation von BÖTTCHER et al. (1989) übereinstimmen. Die vorliegende Simulation liefert somit zwar nicht exakte Ergebnisse, kann aber zumindest die Größenordnung von $\mathrm{T}_{\text {Abbau }}$ richtig abbilden.

Bei den Simulationsergebnissen (Tab. 12) ist folgendes festzustellen: $\mathrm{T}_{\text {Abbau }}$ ist für Sandböden recht gering (i.d.R. < 10 Jahre). Es stellt sich die Frage, ob sich dieser Befund mit der Annahme, die Reduktionsmittelvorräte seien nicht regenerierbar, vereinbaren lässt. Die Flächen werden seit mehreren Dekaden intensiv bewirtschaftet wobei die N-Salden in der Größenordnung der Modellparameter $\left(50-100 \mathrm{~kg} \mathrm{~N} \mathrm{ha}^{-1} \mathrm{a}^{-1}\right)$ liegen dürften. Wenn ein Nitratabbau in der gemessenen Größenordnung unter Freilandbedingungen stattgefunden hätte, müsste das De 
Tab. 12: Ergebnisse der Modellierung des langfristigen Nitratabbaus in den untersuchten Böden

\begin{tabular}{|c|c|c|c|c|c|c|c|c|c|}
\hline & & & \multicolumn{7}{|c|}{ Modellszenarien (1 - 7) } \\
\hline & & & 1 & 2 & 3 & 4 & 5 & 6 & 7 \\
\hline $\mathrm{S}\left(\mathrm{mm} \mathrm{a}^{-1}\right)$ & & & 120 & 60 & 240 & 120 & 120 & 120 & 120 \\
\hline N-Saldo $\left(\mathrm{kg} \mathrm{ha}^{-1} \mathrm{a}^{-1}\right)$ & & & 100 & 100 & 100 & 50 & 200 & 100 & 100 \\
\hline Schichtdicke (m) & & & 1 & 1 & 1 & 1 & 1 & 0,1 & 0,5 \\
\hline Fläche & $\mathbf{R}_{\mathrm{NO} 3}$ & $\mathbf{M}_{\mathrm{NO} 3}$ & \multicolumn{7}{|c|}{ Abbauzeiten in Jahren (Restnitrat in $\mathbf{m g ~ N} \mathrm{I}^{-1}$ ) } \\
\hline Reinshof & 0,52 & 1027 & $154(0)$ & $154(0)$ & $154(0)$ & $308(0)$ & $77(0)$ & $15(0)$ & $77(0)$ \\
\hline Oldendorf & 0,09 & 79,98 & $12(0)$ & $12(0)$ & $12(0)$ & $24(0)$ & $6(0)$ & $5(64)$ & $6(0)$ \\
\hline Vechtel & 0,07 & 38,64 & $6(0)$ & $6(0)$ & $6(0)$ & $12(0)$ & $4(33)$ & $4(70)$ & $4(16)$ \\
\hline Freistatt & 0,04 & 21,20 & $4(10)$ & $4(21)$ & $4(5)$ & $6(0)$ & $4(94)$ & $4(76)$ & $4(47)$ \\
\hline Fuhrberg & 0,01 & 11,31 & $5(53)$ & $5(107)$ & $5(27)$ & $5(12)$ & $5(137)$ & $5(80)$ & $5(68)$ \\
\hline Dinklage & 0,08 & 56,21 & $8(0)$ & $8(0)$ & $8(0)$ & $17(0)$ & $4(10)$ & $4(68)$ & $4(5)$ \\
\hline Markhausen & 0,06 & 65,93 & $10(0)$ & $10(0)$ & $10(0)$ & $20(0)$ & $7(42)$ & $7(71)$ & $7(21)$ \\
\hline Aher Kämpe & 0,47 & 1101 & $165(0)$ & $165(0)$ & $165(0)$ & $331(0)$ & $83(0)$ & $17(0)$ & $83(0)$ \\
\hline Bühren & 0,03 & 10.10 & $2(18)$ & $2(37)$ & $2(9)$ & $3(0)$ & $2(102)$ & $2(77)$ & $2(51)$ \\
\hline Schladen & 0,42 & 618,2 & $93(0)$ & $93(0)$ & $93(0)$ & $185(0)$ & $46(0)$ & $9(0)$ & $46(0)$ \\
\hline Neuhäuser- Felde & 0,23 & 132,34 & $20(0)$ & $20(0)$ & $20(0)$ & $40(0)$ & $10(0)$ & $3(36)$ & $10(0)$ \\
\hline
\end{tabular}


nitrifikationspotential ohne eine Regeneration des Reduktionsmittelvorrats längst erschöpft sein. Folgende Erklärungsmöglichkeiten für diese Diskrepanz bieten sich an:

1. Entgegen der bisherigen Einschätzungen (vgl. Kap. 2.1) findet doch eine Regeneration des Denitrifikationspotentials durch Eintrag von gelöstem Kohlenstoff aus der Wurzelzone statt. SIEMENS et al. (2003) hatten für den $\mathrm{C}_{\text {lös }}$-Eintrag im Inkubationsversuch zwar eine geringe Bedeutung abgleitet. Durch anaerobe Inkubation von Sickerwässern ergab sich ein mittlerer Nitratabbau von ca. $1 \mathrm{mg} \mathrm{N}^{-1}$ Sickerwasser innerhalb von 70 Tagen. Aus diesem Wert lässt sich für eine Lagerungsdichte von $1,5 \mathrm{~g} / \mathrm{cm}^{3}$ eine Denitrifikationsrate von 0,004 $\mathrm{mg} \mathrm{N} \mathrm{kg}^{-1}$ Boden $\mathrm{d}^{-1}$ ableiten. Diese Rate reicht nicht aus, um eine vollständige Regeneration des Denitrifikationspotentials über $\mathrm{C}_{\text {lös }}$-Eintrag zu erklären, da sie deutlich unter den geringsten Raten der Versuchsflächen liegt. Um zu verlässlichen Aussagen zu kommen, wären weiterführende Untersuchungen zur Bedeutung von $\mathrm{C}_{\text {lös }}$ als Reduktionsmittel wünschenswert.

2. Es stellt sich die Frage, ob unter Freilandbedingugnen auf allen Standorten in den untersuchten Schichten überhaupt Denitrifikation stattfindet. Ob dies der Fall ist, hängt im wesentlichen davon ab, ob die betrachteten Schichten im Freiland anaerob sind. Letzteres wäre nur dann der Fall, wenn die mikrobielle Aktivität im oberflächennahen Grundwasser ausreicht, um den Sauerstoff-Eintrag aus der Atmosphäre abzubauen, was bei den $\mathrm{C}_{\text {org }}$-armen Standorten nicht unbedingt der Fall sein muss. Ein direkter Nachweis für Denitrifikation in den untersuchten Schichten liegt für einen Teil der Flächen (Markhausen, Vechtel, Oldendorf) durch in situ-Messungen vor (WELL et al., 2003). Die gemessenen in situ-Raten betrugen $0,014-0,119 \mathrm{mg} \mathrm{N} \mathrm{kg}^{-1} \mathrm{~d}^{-1}$ in Markhausen, 0,014-0,148 $\mathrm{mg} \mathrm{N} \mathrm{kg}^{-1} \mathrm{~d}^{-1}$ in Oldendorf und 0,001-0,022 mg $\mathrm{N} \mathrm{kg}^{-1} \mathrm{~d}^{-1}$ in Vechtel. Während die Größenordnung der in situ-Werte für Oldendorf und Markhausen mit den Laborwerten übereinstimmt, sind die Laborwerte für Vechtel $\left(0,11 \mathrm{mg} \mathrm{N} \mathrm{kg}{ }^{-1} \mathrm{~d}^{-1}\right)$ sehr viel höher und stellen vermutlich eine starke Überschätzung der Denitrifikation unter Freilandbedingungen dar. Da der Vorrat an Reduktionsmitteln in Vechtel deutlich geringer war als in Oldendorf und Markhausen, ist es denkbar, dass in Vechtel die mikrobielle Aktivität nicht ausreichte, um unter Freilandbedingungen ein 
anaerobes Milieu zu schaffen. Dies trifft möglicherweise für alle Flächen mit geringen Reduktionsmittelvorräten (Vechtel, Fuhrberg, Freistatt, Bühren) zu.

3. Die gemessenen Nitratabbauraten sind als Restaktivitäten zu betrachten, da wie zuvor diskutiert - in den Untersuchungsflächen seit langem Nitratauswaschung stattfindet und das ursprüngliche, fossile Denitrifikationspotential bei der Beprobung schon zum Teil erschöpft war. Insofern wäre theoretisch denkbar, dass das im Labor gemessene Rest-Denitrifikationspotential auch unter Freilandbedingungen in den nächsten Jahren vollständig erschöpft wird. Dieser Deutung widerspricht die Einheitlichkeit des Denitrifikationspotentials der Böden mit geringem Reduktionsmittelvorrat, bei denen die Simulation übereinstimmend Abbauzeiten $<10$ Jahre ergeben hat. Es ist sehr unwahrscheinlich, dass diese Flächen sich gleichzeitig in der Endphase der Erschöpfung befinden. Angesichts der Streubreite der gemessenen Gesamtvorräte an $\mathrm{C}_{\text {org }}$ und Sulfid (vgl. Tab. 6) wäre es viel plausibler, wenn auch das Restdenitrifikationspotential weiter streuen würde. Diese Theorie ist als alleinige Erklärung für die kurzen Abbauzeiten deshalb nicht ausreichend.

Zusammenfassend lässt sich feststellen, dass jede der 3 Erklärungstheorien nur als Teilerklärung in Frage kommt. Ob sie zusammengenommen die kurzen Abbauzeiten der Böden mit geringem Reduktionsmittelvorrat erklären können oder ob noch weitere Faktoren eine Rolle spielen, kann anhand der Versuchsergebnisse nicht geklärt werden. Für eine zuverlässigere Bewertung des Denitrifikationpotentials der Böden mit geringem Reduktionsmittelvorrat sind auf jeden Fall weitere Untersuchungen erforderlich.

Die Abauzeiten der Böden mit hohem Reduktionsmittelvorrat (Auen-Gleye) zeigen, dass hier bei $1 \mathrm{~m}$ Schichtmächtigkeit über längere Zeit (ca. 50 - 330 Jahre) mit einem Schutz der Grundwasserressourcen durch vollständigen Nitratabbau in den untersuchten Schichten zu rechnen ist. Da die Denitrifikation in der reaktiven Schicht vollständig ist (kein Restnitrat) hängt die Abbauzeit ausschließlich von der Nitratfracht ab. Der Vergleich zwischen Labor- und in situMessungen der Denitrifkation im oberflächennahen Grundwasser hat gezeigt, dass die Werte für derartige Böden recht gut übereinstimmen (WELL et al., 2003). Bei diesen Böden ist also davon auszugehen, dass die Größenordnung der gemessenen Nitratabbauraten auch für Freilandbedingungen gültig ist. Für die 
wirkliche Größenordnung von $\mathrm{M}_{\mathrm{NO} 3}(\infty)$ ist jedoch die in Kap. 5.5 dikutierte Einschränkung zu berücksichtigen. Da die Werte für $\mathrm{M}_{\mathrm{NO} 3}(\infty)$ Minimalschätzungen für die Zeit nach Ende der Laborinkubationsversuche beinhalten, kann man die in Tab. 12 gezeigten Abbauzeiten auch als Minimalwerte betrachten.

Bei den simulierten Restnitratgehalten werden nur bei den Böden mit geringen Aktivitäten Werte $>0$ vorhergesagt (Tab. 12), während sich für die Böden mit hohen Aktivitäten bei allen Szenarien mit $1 \mathrm{~m}$ Schichtmächtigkeit ein vollständiger Nitratabbau ergibt. Um zu prüfen, ob dieses Muster auch unter Freilandbedingungen zutrifft, wurden die bei der Beprobung der Böden ausgenommenen Nitrat-Tiefenprofile mit der mittleren potentiellen Nitratabbaurate $\left(\mathrm{R}_{\mathrm{NO} 3 p o t, \mathrm{M}}\right)$ der einzelnen Proben verglichen. In Abb. 11 ist dieser Vergleich für alle Einzelproben der Böden unter Ackernutzung gezeigt. Die negative Korrelation zwischen $\mathrm{R}_{\mathrm{NO} \text {, }}$ und Nitratkonzentration ist deutlich erkennbar. Diese Daten zeigen, dass die in der Simulation ermittelte Beziehung zwischen $\mathrm{R}_{\mathrm{NO} 3 \text { pot, }} \mathrm{M}$ und Restnitrat sich auch im Freiland wiederfindet und könnte somit als qualitative Validierung der Simulation betrachtet werden.

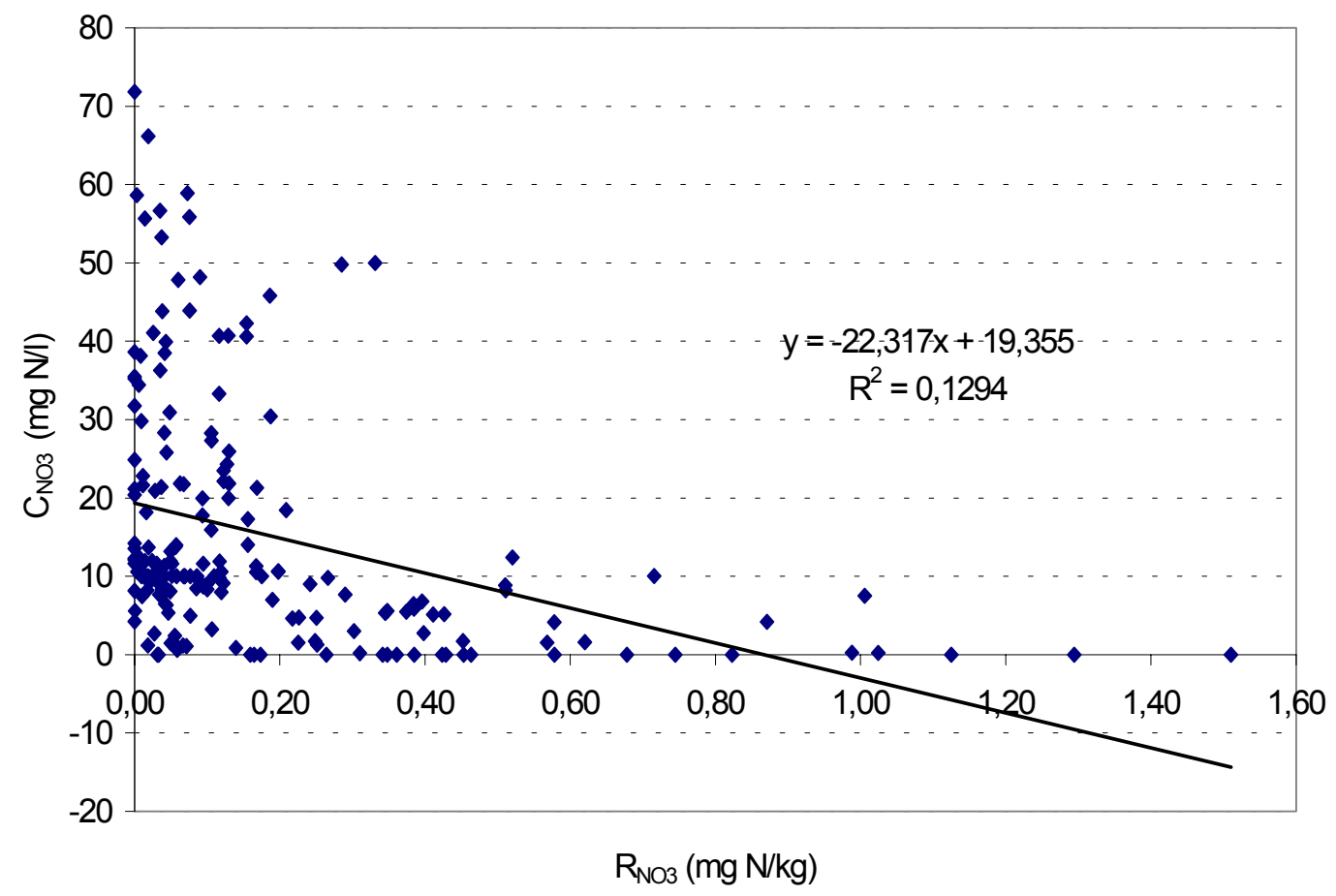

Abb. 11: Nitratgehalte des Bodenwassers $\left(\mathrm{C}_{\mathrm{NO}}\right)$ bei der Probenahme im Feld im Vergleich zur mittleren Nitratabbaurate $\left(\mathrm{R}_{\text {NO3pot, }}\right.$ ) für die Einzelproben der Böden unter Ackernutzung 


\section{Schlussfolgerung und Ausblick}

Durch die abnehmenden Raten der Nitratreduktion bei allen untersuchten Böden im Verlauf der Langzeitinkubation wurde demonstriert, dass unter kontinuierlichem Nitrateintrag mit einer fortschreitenden Erschöpfung des Denitrifikationspotentials zu rechnen ist. Dieser Vorgang wurde in der vorliegenden Arbeit für das oberflächennahe Grundwasser hydromorpher Böden erstmals in größerem Umfang nachgewiesen. Die Ergebnisse liefern somit einen wichtigen Beitrag, um die Vorstellungen vom NHaushalt der Agrarökosysteme in Niederungsgebieten $\mathrm{zu}$ vervollständigen. Für die langfristige Grundwasserqualität ergibt sich die Konsequenz, dass bei gleich bleibender Nitratauswaschung aus der ungesättigten Zone in Zukunft mit steigenden Nitratgehalten in den untersuchten Schichten zu rechnen ist.

Die Streubreite der Gehalte an Reduktionsmitteln für die Denitrifikation $\left(\mathrm{C}_{\text {org }}\right.$, Sulfid $)$ in den untersuchten Böden zeigt, dass das Vorkommen bedeutender Vorräte im oberflächennahen Grundwasser hydromorpher Böden eher der Regel- als der Ausnahmefall ist. Das bedeutet, dass in Niederungsgebieten generell anzunehmen ist, dass ein gewisser Teil des Nitrateintrags im oberflächennahen Grundwasser abgebaut wird. Da der Vorrat an reduzierten Verbindungen endlich ist, muss damit gerechnet werden, dass in diesen Gebieten die Nitratgehalte in Vorflutern bzw. im Förderwasser der Trinkwassergewinnung aufgrund fortschreitender Erschöpfung langfristig ansteigen. Dieser Effekt ist in vielen Flussauen wegen der häufig vergleichsweise hohen Reduktionsmittelvorräte u.U. erst in einigen Jahrzehnten zu erwarten. Für die Gley-Posdole der niederen Geest ist hingegen anzunehmen, dass während der letzten Dekaden in vielen Fällen bereits eine Verminderung des Nitratabbaus stattgefunden hat und dass diese Entwicklung sich z.T. noch weiter fortsetzt, sofern die Erschöpfung des Denitrifikationspotentials bis heute nicht schon vollständig ist.

Anhand einer einfachen Modellrechnung konnte gezeigt werden, dass das gegenwärtige Denitrifikationspotential in den verschiedenen Böden unter kontinuierlicher Nitratauswaschung innerhalb von Zeitspannen von $<10$ Jahren bis max. ca. 200 Jahren verbraucht würde. Unbekannt ist, welche Höhe das langfristige Denitrifikationspotential der untersuchten Flächen ursprünglich hatte, bevor die Nitratauswaschung im Zuge der Intensivierung der Landwirtschaft an Bedeutung gewann. Prinzipiell könnte die Größenordnung des ursprünglichen Denitrifikationspotentials in einer Modellrechnung auf der Basis der Bewirtschaftungsgeschichte der Untersuchungsflächen abgeschätzt wer- 
den. Um einen direkten Beweis für die vergangene Erschöpfung des Denitrifikationspotentials $\mathrm{zu}$ erbringen, wäre es wünschenswert, vergleichende Untersuchungen zwischen Flächen unterschiedlicher Nutzungsgeschichte durchzuführen. Dabei wäre zu prüfen, ob die Unterschiede im Denitrifikationspotential zwischen hoch belasteten Flächen und relativ unbelasteten Referenzflächen nachweisbar sind.

Die Zeitkurven für die Sulfatbildung haben gezeigt, dass der autotrophen Denitrifikation in sulfidhaltigen Böden eine gewisse Bedeutung zukommt. Bei hohen Sulfidgehalten war dieser Reaktionsweg zu Versuchsbeginn dominierend. Für das gesamte langfristige Denitrifikationspotential war jedoch die heterotrophe Denitrifikation in allen Fällen der wichtigere Prozess.

Anhand der Literaturauswertung wurde einer möglichen Regeneration des Denitrifikationspotentials durch Eintrag von gelöstem organischen Kohlenstoff $\left(\mathrm{C}_{\text {lös }}\right)$ eine geringe Bedeutung zugeschrieben. Bei den Böden mit geringstem Denitirifikationspotential ist jedoch nicht auszuschließen, dass dem $\mathrm{C}_{\text {lös}}$-Austrag eine gewisse Rolle zur Aufrecherhaltung eines Restpotentials auf geringem Niveau zukommt. Um dieses verlässlich zu bestimmen, sind gezielte weiterführende Untersuchungen zur Verfügbarkeit von $\mathrm{C}_{\text {lös }}$ wünschenswert. Eine ebenfalls offene Frage ist, ob es zu einer Regeneration der autotrophen Denitrifikation durch Desulfurikation kommen kann. Sulfidbildung durch Desulfurikation nach vollständigem Nitratabbau ist für tieferes Grundwasser belegt (BöTTCHER et al., 1989). Denkbar wäre, dass es in hydromorphen Böden mit vollständigem Nitratabbau saisonal zum Wechsel zwischen Denitrifikation und Desulfurikation kommt. Ob eine solche Zeitdynamik tatsächlich auftritt und ob sie gegebenenfalls für den Nitratabbau von Bedeutung wäre, ist in weiterführenden Studien zu prüfen.

Für die Verfügbarkeit der Vorräte an organischem Kohlenstoff und Sulfid für die Denitrifikation können anhand der Ergebnisse der Dauerinkubationsversuche recht genaue Angaben gemacht werden. Da die Variationsbreite der verfügbaren Anteile bei den meisten Böden nicht sehr groß war (ca. 2 - $7 \%$ des $\mathrm{C}_{\text {org }}$-Vorrats, ca. 10 - $50 \%$ des Sulfidvorrats), kann anhand der Gesamtvorräte schon die Größenordnung des langfristigen Denitrifikationspotentials abgeschätzt werden. Für den organischen Kohlenstoff ist ungeklärt, wie die verfügbare Fraktion chemisch charakterisiert ist. Da der Anteil des gelösten und des heißwasserlöslichen Kohlenstoffs nur sehr bedingt mit der verfügbaren Fraktion korreliert war, wäre es sinnvoll, in weiterführenden Untersuchungen nach Messgrößen zu suchen, die die verfügbare $\mathrm{C}_{\text {org }}$-Fraktion besser repräsentieren. 
Die vorliegenden Ergebnisse liefern verschiedene Optionen für eine Prognose des langfristigen Denitrifikationspotentials, die als Hilfsmittel für die Planung und Analyse in den Bereichen Wasserversorgung und Umweltsteuerung Verwendung finden können. Für eine grobe Einstufung kann die durchschnittliche Verfügbarkeit des organischen Kohlenstoffs und des Sulfids verwendet werden. Unter Hinzunahme von Bodentyp, Textur und pH-Wert können die in dieser Arbeit ermittelten Regressionsmodelle für eine präzisere Prognose verwendet werden. Sinn und Zweck der Durchführung derartiger Studien bestehen darin, das langfristige Gefährdungspotential der Grundwasserqualität zu kartieren, um dadurch die Effizienz von Schutzmaßnahmen zu maximieren. Beispielsweise können sensible Areale ausgewiesen werden, für die besondere Schutzmaßnahmen zu erwägen sind. Auf keinen Fall sollte das zur Zeit noch vorhandene Denitrifikationspotential jedoch als Senke im Hinblick auf eine problemlose Entsorgung von Nitratausträgen aus der landwirtschaftlichen Bodennutzung betrachtet werden. Der Vorrat an reduzierten Stoffen im Grundwasser stellt eine endliche Ressource dar, die für die langfristige Sicherung der Grundwasserqualität auch für künftige Generationen zu erhalten ist. Das umweltpolitische Ziel, Nitratausträge aus dem durchwurzelten Boden in tiefere Bodenschichten und ins Grundwasser zu minimieren, ist deshalb auch für hydromorphe Mineralböden uneingeschränkt gültig. 


\section{Zusammenfassung}

Im oberflächennahen Grundwasser hydromorpher Böden spielt die Denitrifikation eine bedeutende Rolle für den Abbau von Nitrat und die Bildung von Lachgas. Je nach Vorkommen von fossilen oder rezenten Reduktionsmitteln können die potentiellen Denitrifikationsraten in sehr weiten Grenzen schwanken. Es besteht die Befürchtung, dass permanente Nitrateinträge langfristig zu einer Erschöpfung der Vorräte an fossilen Reduktionsmitteln für die Denitrifikation (organischer Kohlenstoff, Sulfid-Schwefel) und somit zu abnehmenden Raten des denitrifikativen Nitratabbaus führen. Dies würde letztlich einen Anstieg der Nitratgehalte im Grundwasser bewirken. Die Höhe der Gesamtvorräte an Sulfid-Schwefel und organischem Kohlenstoff im oberflächennahen Grundwasser hydromorpher Böden sowie deren Verfügbarkeit für die Denitrifikation sind bisher noch unzureichend erforscht.

Ziel der vorliegenden Arbeit war es, die langfristige Dynamik der Denitrifikation in der gesättigten Zone hydromorpher Mineralböden unter landwirtschaftlicher Nutzung mit kontinuierlicher Nitratauswaschung zu beschreiben. Dies beinhaltet zunächst die Quantifizierung der Gesamtvorräte an reduzierten Verbindungen (organischer Kohlenstoff, reduzierte Schwelfelverbindungen), die als Elektronendonatoren für die Denitrifikation eine wesentliche Rolle spielen sowie die Bestimmung ihrer langfristigen Verfügbarkeit für die Denitrifikation. Darüber hinaus sollte die Zeitdynamik des Nitratabbaus durch Denitrifikation während einer fortschreitenden Erschöpfung der verfügbaren Reduktionsmittel der Böden bestimmt werden. Schließlich sollte eine Grundlage geschaffen werden, um das langfristige Denitrifikationspotential im oberflächennahen Grundwasser hydromorpher Böden auf der Basis einfach zu bestimmender Bodenparameter vorherzusagen.

Das Versuchsprogramm umfasste insgesamt 11 in Niedersachsen gelegene Böden (Gley-Podsole, Auen-Gleye, Auen-Pseudogley, Tiefumbruch-Boden und Marsch), die im Hinblick auf den Bodentyp, den Vorrat an Reduktionsmitteln, die Textur und den Grundwasserflurabstand das Spektrum der landwirtschaftlich genutzten hydromorphen Mineralböden der Region zu einem großen Teil abdecken.

Aus dem Untergrund der Untersuchungsflächen wurden im oberflächennahen Grundwasser sowie im Kapillarsaum in Intervallen von $10-20 \mathrm{~cm}$ Bodenproben entnommen, wobei sich der beprobte Bereich zwischen mindestens $1 \mathrm{~m}$ und höchstens $4 \mathrm{~m}$ unter der 
Geländeoberkante befand. Neben der Bestimmung bodenkundlicher Basiskenngrößen (Korngrößenspektrum, pH, Kalkgehalt) wurden die Gehalte an organischem Kohlenstoff (Gesamtmenge, gelöste und heißwasserlösliche Fraktion) und Schwefel (Gesamt-, Sulfid- und Sulfat-Schwefel) gemessen.

Für die Bestimmung des langfristigen Denitrifikationspotentials erfolgte eine Langzeitinkubation der Bodenproben unter denitrifizierenden Bedingungen. Dazu wurden Bodensuspensionen mit $\mathrm{K}^{15} \mathrm{NO}_{3}$ versetzt und bis zu 700 Tage lang in gasdichten Flaschen bei $25^{\circ} \mathrm{C}$ anaerob inkubiert. Zur Bestimmung des Temperatureinflusses wurden ein Teil der Proben zusätzlich bei $10^{\circ} \mathrm{C}$ inkubiert. Die Flaschen wurden während der Inkubation mehrfach auf Nitrat, Sulfat in der Bodenlösung und ${ }^{15}\left(\mathrm{~N}_{2}+\mathrm{N}_{2} \mathrm{O}\right)$ in der Gasphase analysiert, um den Zeitgang der Denitrifikation und der Sulfatproduktion zu ermitteln. Zusätzlich erfolgte eine aerobe Langzeitinkubation von Parallelproben zur Bestimmung des oxidierbaren Sulfids.

Die Vorräte an organischem Kohlenstoff $\left(\mathrm{C}_{\text {org }}\right)$ und Sulfid schwankten sowohl zwischen den verschiedenen Böden als auch innerhalb der einzelnen Tiefenprofile in weiten Grenzen. Die vertikale Verteilung war in einigen Fällen durch nach unten hin zunehmende Vorräte gekennzeichnet, während in anderen Fällen Maxima unmittelbar unterhalb der Grundwasseroberfläche vorhanden waren. Einige der Gley-Podsole sowie der Auen-Pseudogley waren sulfidfrei und enthielten nur geringe $\mathrm{C}_{\mathrm{org}}$-Vorräte $(<0,2 \%)$. Andere Gley-Podsole wiesen $\mathrm{C}_{\text {org }}$-Gehalte bis ca. $1 \%$ auf und enthielten in den unteren Tiefen geringe Sulfidmengen. Der Marschboden war durch mäßige $\mathrm{C}_{\text {org }}$-Gehalte, jedoch hohe Sulfidvorräte (bis $1 \mathrm{mg} \mathrm{kg}^{-1}$ ) gekennzeichnet. Die Auen-Gleye wiesen übereinstimmend hohe Gehalte sowohl an $\mathrm{C}_{\text {org }}$ (bis ca. $10 \%$ ) als auch an Sulfid (bis ca. $8 \mathrm{mg}$ $\mathrm{kg}^{-1}$ ) auf.

Während der anaeroben Inkubation zeigten alle Proben eine im Zeitverlauf abnehmende Rate der Nitratreduktion $\left(\mathrm{R}_{\mathrm{NO} 3}\right)$. Bei den $\mathrm{C}_{\text {org }}$-armen Gley-Podsolen waren die Raten insgesamt gering $\left(<0,2 \mathrm{mg} \mathrm{N} \mathrm{kg}^{-1} \mathrm{~d}^{-1}\right)$ und kamen bis zum Ende der Inkubation zum Erliegen. Bei den Böden mit hohen Reduktionsmittelvorräten betrugen die Nitratabbauraten bis zu 2,3mg $\mathrm{N} \mathrm{kg}^{-1} \mathrm{~d}^{-1}$ und fielen bis zum Versuchsende bis auf ca. ein Drittel der Anfangsaktivität ab. In allen sulfidhaltigen Böden war autotrophe Denitrifikation durch Produktion von Sulfat während der Inkubation nachweisbar. Dabei nahm die Rate der Sulfatproduktion schneller ab als die Rate der Nitratreduktion. Bei hohen Sulfidgehalten dominierte zu Versuchsbeginn die autotrophe über die heterotrophe Denitrifikation. 
Bezogen auf die gesamte Inkubationszeit war jedoch die heterotrophe Denitrifikation in allen Fällen der wichtigere Prozess.

Durch Integration der Zeitkurven der Nitratreduktion und Sulfatproduktion wurde das langfristige Potential der Gesamtdenitrifikation $\left(\mathrm{M}_{\mathrm{NO} 3}\right)$ sowie der autotrophen Denitrifikation $\left(\mathrm{M}_{\mathrm{SO} 4}\right)$ bestimmt. Die Flächenmittelwerte für $\mathrm{M}_{\mathrm{NO} 3}$ betrugen zwischen 10 und 38

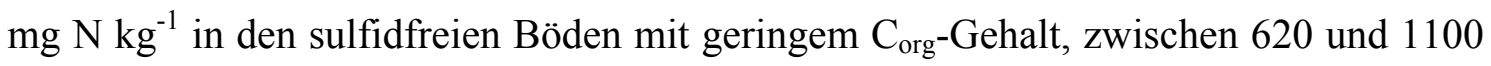
$\mathrm{mg} \mathrm{N} \mathrm{kg}{ }^{-1}$ in den Auen-Gleyen und zwischen 56 und $132 \mathrm{mg} \mathrm{N} \mathrm{kg}^{-1}$ in den übrigen Böden. Der Anteil der autotrophen Denitrifikation am langfristigen Denitrifikationspotential in den sulfidhaltigen Böden lag zwischen 12 und $32 \%$.

Die Verfügbarkeit des Reduktionsmittelvorrats für die Denitrifikation wurde aus dem Verhältnis von $\mathrm{M}_{\mathrm{NO} 3}$ zum Gesamtvorrat an (Sulfid $+\mathrm{C}_{\text {org }}$ ) bzw. von $\mathrm{M}_{\mathrm{SO} 4}$ zum Gesamtvorrat an Sulfid abgleitet. In den meisten Böden war der Sulfidvorrat zu einem großen Teil (ca. $10-50 \%$ ), der (Sulfid $+\mathrm{C}_{\text {org }}$ )-Vorrat nur zu einem geringen Teil (ca. 2 - 7 \%) verfügbar. Die Sulfidverfügbarkeit war unter Aerobie deutlich höher als unter Anaerobie.

Die Umsatzgrößen $\mathrm{M}_{\mathrm{SO} 4}, \mathrm{M}_{\mathrm{NO} 3}, \mathrm{R}_{\mathrm{NO} 3}$ waren mit den meisten chemisch-physikalischen Bodenparametern, insbesondere jedoch mit den Gehalten an $\mathrm{C}_{\text {org }}$ und Sulfid, signifikant korreliert. Gleiches gilt für die Verfügbarkeit des Sulfids, nicht aber für die Verfügbarkeit von (Sulfid $+\mathrm{C}_{\text {org }}$ ).

Durch multiple Regressionsanalyse wurden empirische Prognosemodelle für die verschiedenen Umsatzgrößen abgeleitet. Beim Vergleich gemessener und mittels Regressionsgleichungen berechneter Werte zeigte sich, dass die Prognose von Einzelwerten für alle Messgrößen unbefriedigend ist. Die berechneten Flächenmittelwerte für $\mathrm{M}_{\mathrm{SO}}$, $\mathrm{M}_{\mathrm{NO} 3}, \mathrm{R}_{\mathrm{NO} 3}$ sowie für die Verfügbarkeit des Sulfidvorrats zeigten hingegen recht gute Übereinstimmungen mit den Messwerten.

Zur Prognose der langfristigen Grundwasserqualität in den untersuchten Böden wurde ein stark vereinfachendes Transport- und Umsatzmodell verwendet. Als Eingabedaten wurden die Messwerte des langfristigen Denitrifikationspotentials und der mittleren Nitratabbaurate sowie verschiedene Annahmen für die Grundwasserneubildung, die jährliche Nitratfracht und die Mächtigkeit der reaktiven Schicht verwendet. Die Simulationsergebnisse zeigten, dass mit einer vollständigen Erschöpfung des Denitrifikationspotentials innerhalb eines Zeitraums von $<10$ Jahren (Böden mit geringen Reduktionsmittelvorräten) bis $>200$ Jahren (Auen-Gleye mit hohen Reduktionsmittelvorräten) zu rechnen ist. 
In der vorliegenden Arbeit wurde die Erschöpfung des Denitrifikationspotentials durch kontinuierlichen Nitrateintrag im oberflächennahen Grundwasser hydromorpher Mineralböden erstmals in größerem Umfang nachgewiesen und quantifiziert. Für die langfristige Grundwasserqualität ergibt sich die Konsequenz, dass bei gleich bleibender Nitratauswaschung aus der ungesättigten Zone in der Zukunft mit steigenden Nitratgehalten in den untersuchten Schichten zu rechnen ist. Dies wird in vielen Flussauen wegen der häufig vergleichsweise hohen Reduktionsmittelvorräte u.U. erst in einigen Jahrzehnten der Fall sein. Für die Gley-Podsole der niederen Geest ist hingegen anzunehmen, dass während der letzten Dekaden bereits eine Verminderung der Nitratabbaurate stattgefunden hat und dass diese Entwicklung sich z.T. noch weiter fortsetzt. In einigen Fällen ist davon auszugehen, dass das Denitrifikationspotential schon gegenwärtig nahezu vollständig erschöpft ist.

Die heute noch verbliebenen endlichen Ressourcen an Reduktionsmitteln in hydromorphen Mineralböden sind für die langfristige Sicherung der Grundwasserqualität bedeutend und ist deshalb möglichst vor weiterem Abbau durch Nitrateinträge zu schützen.

Die vorliegenden Ergebnisse liefern verschiedene Optionen für eine Prognose des langfristigen Denitrifikationspotentials. Sie können somit als Hilfsmittel bei der Planung und Analyse in den Bereichen Wasserversorgung und Umweltsteuerung Verwendung finden, um die langfristige Gefährdung der Grundwasserqualität in Niederungsgebieten zu kartieren. 


\section{Literatur}

Abou-SEADA, M N. I. (1986): Quantitative und qualitative Zusammensetzung der Stickstoffentgasung (Denitrifikation) verschiedener denitrifizierender Organismen in Abhängigkeit von der Nitratkonzentration, dem $\mathrm{pH}-$ Wert, dem Sauerstoffpartialdruck und unterschiedlichen Böden. Diss. Univ. Hohenheim.

Abou-Seada M.N.I. \& J.C.G. OtTow, (1988): Einfluß chemischer Bodeneigenschaften auf Ausmaß und Zusammensetzung der Denitrifikationsverluste drei verschiedener Bakterien. Z. Pflanzenern. Bodenkunde 151, S. 109-115.

Adriano, D.C., P.F. Pratt \& F.H. TAKATORi (1972): Nitrate in unsaturated zone of an alluvial soil in relation to fertilizer nitrogen rate and irrigated level. J. Environ. Qual. 1 S. 418-422.

ArAH, J.R. M. \& K.A. SMith (1990): Factors influencing the fraction of gaseous products of soil denitrification evolved to the atmosphere as nitrous oxide. In: BOUWMAN, A.F. (Hg.): Soils and the greenhouse effect. New York: John Wiley. S. 475 - 480 .

Artiola, J. F.(1997): Denitrification activity in the vadose zone beneath a sludgeamended semi-arid soil: Communications in Soil Science and Plant Analysis, V. 28 , p. $797-812$.

AulaKH, M.S., J.W. Doran \& A.R. Mossier (1992): Soil denitrification - significance, measurement, and effect of management. Adv. Soil Sci. 18, p. 1-57.

BAiLEy, L. D. \& E. G. BeAuchamp (1973): Effects of temperature on $\mathrm{NO}_{3}{ }^{-}$and $\mathrm{NO}_{2}{ }^{-}$ reduction, nitrogenous gas production, and redox potential in a saturated soil. Can. J. Soil Sci. 53, p. 213-218.

Beauchamp, E.G., C. Gale \& J.C. Yeomans (1980): Organic matter availability for denitrification in soils of different textures and drainage classes. Comm. Soil Sci. Plant Anal. 11, p. 1221 - 1233.

BECKER, K.-W., von E. BEÖCZY, \& B. SCHÄFER, (1988a): Denitrifikation beim Nitrattransport in mächtigen Lößdecken: Steuerung durch organische Bodeneluate und die Höhe des Grundwasserspiegels. Mitt. Dt. Bodenkdl. Ges. 57, S. 147-152. 
Becker, K.-W., H. HöPer \& B. Meyer (1992): Kritik der Acetylen-InhibitionsMethode in ihrer Anwendbarkeit auf Messungen der Denitrifikation im Felde Vergleich mit der ${ }^{15} \mathrm{~N}$-Bilanz - und der ${ }^{15} \mathrm{~N}$-Gas-Freisetzungs-Methode. Göttinger Bodenkundl. Ber. 96, S. 116-343.

BEHM, R. (1988): Untersuchungen zur Bestimmung der leicht umsetzbaren N- und CAnteile im Heißwasserextrakt des Bodens - Kurzmitteilung. Arch. Acker- Pflanzenbau Bodenkd., Berlin 32 (1988) 5, S. 333-335.

BENCKISER, G. (1996): In situ Bestimmung von Denitrifikationsverlusten auf unterschiedlichen landwirtschaftlichen Nutzflächen mit Acetylen- Inhibierungstechnik. VDLUFA- Schriftr. 41 Habilitationsschrift, Darmstadt, $121 \mathrm{~S}$.

BHOGAL, A., \& M. SHEPHERD (1997): Effect of poultry manure on the leaching of carbon from a sandy soil as a potential substrate for denitrification in the subsoil: Journal of the Science of Food and Agriculture, V. 74, p. 313-322.

Blackmer, A.M. \& Bremner, J.M. (1978): Inhibitory effect of nitrate on reduction of $\mathrm{N}_{2} \mathrm{O}$ to $\mathrm{N}_{2}$ by soil microorganisms. Soil Biol. Biochem. 10, S. 187 - 191.

BÖTTCHER, J. (1992): Stoffanlieferung in das Grundwasser bei Sandböden und Stoffumsetzungen in einem Lockergesteins-Aquifer. Habilitationsschrift, Hannover, 118 S..

Böttcher, J., O. Strebel \& M. Duysniveld (1985): Vertikale Stoffkonzentrationsprofile im Grundwasser eines Lockergesteins-Aquifers und deren Interpretation (Beispiel Fuhrberger Feld). Z. dt. geol. Ges. 136, S. 543-552.

Böttcher, J., O. Strebel \& M. Duysniveld (1989): Kinetik und Modellierung gekoppelter Stoffumsetzungen im Grundwasser eines Lockergesteins-Aquifers. Geol. Jahrbuch Reihe C, H. 51, S. 1-40.

BowmAn, R.A. \& D.D. FochT (1974): The influence of glucose and nitrate concentrations upon denitrification rates in sand soils. Soil Biol. Biochem. 6, S. 297-301.

BOUWMAN, A. F (1990): Exchange of greenhouse gases between terrestrial ecosystems and the atmosphere in A. F. BouWMANN (ed): Soils and the greenhouse effect. John Wiley and sons Ltd., Chichester, p. 61-127.

Bremner, J. M. \& K. SHAw, (1958): Denitrification in Soils. I. Methods of investigation. II. Factors affecting denitriffication. J. Agric. Sci. 51, p. 85-88.

BRUMSACK, H.-J.(1981): A Simple Method for the Determination of Sulfide- and Sulfat-Sulfur in Geological Materials by using different Temperatures of Decomposition. Fersenius Z. Anal. Chem., 307, p. 206-207. 
Buresh, R. J., M.I. SAMSON \& S.K. DedatTa (1993): Quantification of denitrification in flooded soils as affected by rice establishment method. Soil Biol. Biochem. 25, 7, p. 843-848.

BURFORD J.R. \& J.M. BREMNER, (1975): Relationships between denitrification capacities of soils and total water soluble and readily decomposable soil organic matter. Soil Biol. Biochem. 7, p. 389-394.

Burth J. \& J.C. G OtтоW (1982): Stickstoffentgasung bei verschiedenen denitrifizierenden Bakterien und Fusarium solani in Abhängigkeit von der Wasserstoffionenkonzentration (pH). Landwirt. Forsch. Sonderh. 38, S. 655-666.

Christensen S. \& J.M. Tiedje (1988): Denitrification in the field, analysis of spatial and temporal variability. In: D.S. Jenkinson \& K.A. Smith (eds.), Nitrogen Efficiency in Agricultural Soils, p. 295 - 301, Elsevier, London.

Cho C.M. \& L. SAKDinAN (1978): Mass spectrometric investigation on denitrification. Can. J. Soil Sci. 58, p. 443-457.

Chalamet A. (1985): Effects of enviromental factors on denitrification. In: Denitrification in the nitrogen cycle. (Ed.) H.L. Goltemann, Nato Conference Series I, 9, p. 7-29.

CORDT, T. \& H. KußMAUl (1990): Niedermolekulare Carbonsäuren im Boden, in der ungesättigen Zone und im Grundwasser. Vom Wasser 74, S. 287-298.

Colbourm P. \& R.J. Dowdell (1984): Denitifikation in field soils. Plant and Soil 76, p. $213-225$.

COPPER G.S. \& R.L. SMITH (1963): Sequence of products formed during denitfication in some diverse western soils. Soil Sci. Soc. Am. Proc. 27, p. 659-662.

CURTIN D., \& J. K.SYERS (1990): Extractability and adsorption of sulphate in soils. J. Siol Sci. 41, p. 305-312.

DAVIDSON, E.A. (1991): Fluxes of nitrous oxide and nitric oxide from terrestrial ecosystems. In: Rogers, J.E. (Hg.), Microbial-production-and-consumption-ofgreenhouse-gases:-methane,-nitrogen-oxides,-and-halomethanes; American Society for Microbiology; Washington, p. 219-235.

Desmarais, D. J. \& J. M. HAYES, (1976): Tube crackter for opening glass-sealed ampoules under vacuum. Analytical Chemistry 48, p.1651-1652. 
DRECHSLER, H. (1992): Reduktion des Stickstoffs aus dem Überschuß-N-Eintrag agrarischer Ökotope beim Transport durch die Böden ins Grundwasser. Diss. Univ. Göttingen.

Duynisveld W.H.M., O. STRebel \& J. BÖtTCheR (1993): Prognose der Grundwasserqualität in einem Wassereinzugsgebiet mit Stofftransportmodellen. Texte 5/93, Umweltbundesamt, Berlin.

Eriksen, A.B. \& Holtan-Hartwig, L. (1993): Emission spectrometry for direct measurements of nitrous oxide and dinitrogen from soil. Siol Sci. Soc. Am. J. 57, p. $738-742$.

El-Demerdash M.E. \& J. C. G. OtTOW (1983): Einfluß einer hohen Nitratdüngung auf Kinetik und Gaszusammensetzung der Denitrifikation. Z. Pflanzenernähr. Bodenkd., 146 (2), S. 138-150.

FILLERY I.R.P. (1983): Biological denitrification. In: Freney, J.R., Simpson, J.R., Gaseous loss of nitrigen from soil-plant systems, Nijhoff/Junk, The Hague, p. 33-64.

FISCHER M. (1989): Schwefelvorräte und Bindungsformen süddeutscher Waldböden in Abhängigkeit von Gestein und atmogener Schwefeldeposition. Schriftenreihe der forstwiss. Fak. der Univ. München und der bayer. forstlichen Versuchs- und Forschungsanstalt, Band 100.

Firestone M.K., M.S., SMith \& J.M., TiedJE (1979): The influence of nitrate, nitrite and oxygen on the composition of the gaseous products of denitrification in soil. Soil Sci Soc. Am. J. $\underline{43}$, p. 1140 - 1144.

Firestone M.K., R. B. Firestone \& J.M., Tiedje (1980): Nitrous oxide from soil denitrification: Factors controlling its biological production. Sci. 208. p. 749-751.

FLESSA H. \& F. BEESE (1995): Effects of sugarbeet residues on soil redox potential and nitrous oxide emission. Soil Sci. Soc. Am. J., 59, S. 1044-1051.

FOCHT, D.D. (1974): The effect of temperature, $\mathrm{pH}$ and aeration on the production of nitrous oxide and gaseous nitrogen - a zero-order kinetic model. Soil Sci. 118 (3), 173-179.

FocHT, D.D. (1978): Methods for analysis of denitrification in soils. In: Nitrogen in the environment, Vol. 2: Soil-Plant Nitrogen Relationships (D.R. NIELSON \& J.G. MacDonald, Eds.). Academic Press, New York, p. 433 - 490. 
FochT, D.D. (1981): Soil denitrification. - In: Genetic engineering of symbiotic nitrogen fixation and conservation of fixed nitrogen. J.M, LyONS, R.C. VALENTINE, D.A. Phillips, D.W. Rains and R.C. Huffaker (Eds.), Plenum Publishing Corporation, p. 499-516.

Frede, H. G., H. Gebhardt \& B. Meyer (1975): Größe, Ursachen und Bedingungen von Boden- und Dünger-N-Verlusten durch Denitrifikation aus dem Ap-Horizont einer Accker-Parabraunerde aus Löss. - Gött. Bodenkdl. Ber. 34, S. 69-213.

Frind E.O., W.H.M., DuYnisveld, O., Strebel \& J. BötTcher (1990): Modeling of multicomponent transport with microbial transformation in groundwater; the Fuhrberg case. Water Resources Research 26, p. 1707 - 1719.

FunK R. (1993): Verlagerung und Abbau von Nitrat in tieferen Bodenschichten süddeutscher Ackerstandorte. Diss. TU. München, 165 S.

Gäth S., , F. Antony, K.W. Becker, H. Geries, H. HöPer, C. Kersebaum, \& R. NiEDER, (1997): Bewertung des standörtlichen Denitrifikations- und Mineralisations/ Immobilisations-Potentials von Böden. Mitt. Dt. Bodenkdl. Ges. 85 III, S. 1373-1376.

GERMON J.C. (1985): Microbiology of denitrification and other processes involving the reduction of oxygenated nitrous compounds. In: H.L. GOLTERMANN (ed): Denitrification in the nitrogen cycle. Nato Conference Series 9, p. 31-46.

Chalk P.M. \& C.J. Smith (1983): Chemodenitrification. In:Gaseous loss of nitrigen from plantsoil systems. (Eds.) J. R. Freney \& J. R. Simpson, The Hague, p. 6589.

GRANLI T. \& O.C. BOCKMAN (1994): Nitrous oxide from agriculture. Norwegian Journal of Agricultural Sciences A, Norsk Hydro Research Centre, Porsgunn Norway.

Heinemeyer O., K. HAider \& A.R. Mosier (1988): Einfluss kontrollierter Wasserzufuhr zu mit Mais bepflanztem Boden auf $\mathrm{N}_{2} \mathrm{O}$ - und $\mathrm{N}_{2}$-Freisetzung aus ${ }^{15} \mathrm{~N}$-Nitrat; Kontinuierliche Redoxpotentialmessung in verschiedenen Bodentiefen. Mitt. Dt. Bodenkdl. Ges. 56, S. 283-288.

HofFMANn M. (2000): Mikrobielle Biomasse und Aktivität in landwirtschaftlich genutzten Böden Niedersachsens, Eine statistische Auswertung von Daten der Bodendauerbeobachtung. Diss. Univ. Göttingen, $125 \mathrm{~S}$.

HofFMANN A. (1991): Veränderung des Nitratabbauvermögens tieferer Bodenschichten durch Stickstoffüberversorgung. - Texte 1, Umweltbundesamt, $160 \mathrm{~S}$. 
KAHLIL M.A.K. \& R.A., RASMUSSEN (1988): Nitrous oxide: Trends and global mass balance over the last 3000 years, Ann. Glaciology, 10, p. 73-79.

Klemedtsson L., B.H. Svensson, T. LindberG, \& T. Rosswall (1977): The use of acetylene inhibition of nitrous oxide reductase in quantifying denitrification in soils. Swed. J. Agric. Res. 7, 179-185.

KNowels R. (1982): Denitrification. Microbiol. Rev. 46, p. 43 - 70.

Kölle W., P. Werner, O.Strebel, \& J. Böttcher (1983): Denitrifikation in einem reduzierenden Grundwasserleiter. Vom Wasser, 61, S. 125-147.

KÖLle W., O. STREBel, \& J. BÖtTCHER (1985): Formation of sulfate by microbial denitrification in a reducing aquifer. Water supply 3, p. $35-40$.

KÖLLE W. (1987): Auswirkungen der Nitratbelastung in einem reduzierenden Grundwasserleiter. DVGW-Schriftenreihe Wasser 31, S. 109-126.

Kölle W., O. Strebel, J. BötTCher (1987): Reduced sulfur compunds in sandy aquifers and their interaction with groundwater. Proc. of the Dresden Symposium, March 1987, „Groundwater Monitoring and Management“, IHAS Publication 173 , p. $23-30$.

KÖLlE W. (1987/1988): Natürliche Denitrifikation durch Schwefel-EisenVerbindungen im aquifer. In: Handbuch Wasserversorgungs- und Abwassertechnik. Vulkan-Verlag, Essen, S. 183-187.

KÖLlE W., O. STREBel \& J. BÖTTSChER (1990): Reduced sulfur compounds in sandy aquifers and their interactions with groundwater. Proc. of the Dresden Symposium: Groundwater Monitoring and Management. IAHS Publ. 173, 23-30.

KöLLE W. (1999): Einfluß des Grundwasserleiters auf die Grundwasserbeschaffenheit aus der Sicht des Wasserchemikers, das Stoffdepot-Konzept, Dresden, S. 69-82.

Kuntze H., G. Roeschmann \& G. SchwerdtFeger (1994): Bodenkunde, 5. Auflage, Ulmer, UTB, Stuttgart.

LEFFLAAR P.A. (1986): Dynamics of partial anaerobiosis, denitrification, and water in soil aggregate: Experimental. Soil Sci. 142, p. 352-362.

LENSI R. \& A. CHAlAmet, (1982): Denitrification in waterlogged soils: In situ temperature-dependent variations. Soil. Biol. Biochem. 14, p. 51-55. 
LEuCHS, W. (1988): Vorkommen, Abfolge und Auswirkungen anoxischer Redoxreaktionen in einem pleistozänen Porengrundwasserleiter. Bes. Mitteilg. Dtsch. Gewässerkundl. Jahrbuch 52, $106 \mathrm{~S}$.

LiEDTKE H. (1984): Namen und Abgrenzungen von Landschaften in der BRD gemäß der amtlichen Übersichtskarte 1: 500 000. Zentralausschuss für deutsche Landeskunde, Selbstverlag.

Lind A.M. \& M.B. Pedersen (1976): Nitrate reduction in the subsoil. Tidsskr. Planteavl. 80 , p. $82-106$.

LiND A.M. (1980): Denitrification in the root zone. Tidsskr. Planteavl. 84, p. 101-110.

MALINOWSKi P. \& J.C.G. OTTOW (1985): Ökologische Bedingungen der Denitrifikation bei Pilzen. Landwirtsch. Forsch. 38, S. 30-34.

MAIDL F.X. \& G. FISCHBECK (1987): Nitratgehalte tiefer Bodenschichten bei unterschiedlichen Fruchtfolgen auf intensiv genutzten Ackerbaustandorten. Z. Pflanzenernähr. Bodenkd. 150, S. 213-219.

MCCARTY G.W. \& J.M. BREMNER (1993): Factors affecting the availability of organic carbon for denitrification of nitrate in subsoils. - Biol. Fertil. Soils 15, p. 132-136.

MCCARTy G.W. \& J.M. BREMNER (1992): Availability of organic carbon for denitrification of nitrate in subsoils. Biol. Fertil. Soils 14, p. 219-222.

MELin J.A. \& H. NOMMiK (1983): Denitrification measurements in intact soil cores. Acta Agriculturae Scandinavica 33, p. 145-151.

MÜCKENHAUSEN E. (1993): Die Bodenkunde und ihre geologischen geomorphologischen mineralogischen und petrologischen Grundlagen. DLG-Verlag, Frankfurt am Main.

Müller M. M., V. Sundman \& J. Skujins (1980): Denitrification in low pH Spodosols and peats determined with the acetylene inhibition method. Appl. Environm. Microbiol. 40, p. 235-239.

Mülller-Thomsen U, U. Pfisterer \& H.P. Blume (1995): Gas-, Wasser- und Stoffdynamik im Deichvorland Nordfrieslands am Beispiel einer typischen Salzmarsch und eines marinen Übergangswatts. Mitt. Dt. Bodenkdl. Ges. 76, S. 525 - 528. 
MunCh J.C. \& J.C.G. OtTOW (1983): Reductive transformation mechanism of ferric oxides in hydromorphic soils. In: Environmental Biogeochemistry. (Ed.) R. Halbery. Ecol. Bull. (Stockholm) 35, S. 383-394.

Munch J.C. \& J.C.G. OTTOW (1987): Einfluss von Bodenfeuchte und Sauerstoff auf die Lachgasbildung denitrifizierender Bakterien. Mitt. Dt. Bodenkdl. Ges. 55, II, S. 505-510.

Nieder R., G. SchOlLmeyer, \& J. Richter (1989): Denitrification in the rooting zone of cropped soil with regard to methodology and climate, a review. Biology and Fertility of Soils 8, p. 219-226.

NiLSEN T.H., R. Well \& D.D. MyROLD (1997): Combination probe for ${ }^{15} \mathrm{~N}$ labelling and measurement of ${ }^{15} \mathrm{~N}$ atom fraction, water potential, and denitrification activity in sub-soil. Soil Science Soc. Am. J. 61, p. 802 - 811.

NÖMMIK H. (1956): Investigations on denitrification in soil. Acta Agric. Scand. 6, p. $195-228$

NÖMmIK H., D. J. PluTh \& J. Melin (1984): Dissimilatory reduction of ${ }^{15} \mathrm{~N}$-labeled nitrate in the presence of nonlabeled $\mathrm{NO}$ or $\mathrm{N}_{2} \mathrm{O}$. Can. J. Soil Sci. 64, p. 21-29.

OBERMANN P. (1990): Significance of anoxic reaction zones in an aquifer in the lower Rhine region. Mitt. Dt. Bodenkdl. Ges. 60, p. 249 - 258.

OBERMANN P. (1988): Der $\mathrm{NO}_{3}$-Eintrag in das Grundwasser und der $\mathrm{NO}_{3}$-Abbau im Grundwasserleiter. UBA Texte 6/88: Grundwasserschutz und N-Düngung, Berlin, S. 35-63.

OBERMANN P. (1981): Hydrochemische / hydromechanische Untersuchungen zum Stoffgehalt von Grundwasser bei landwirtschaftlicher Nutzung. Besondere Mitt. zum Dt. Gewässerkundl. Jahrb., 42, Bonn.

PARKIN T.B., A.J. SESTONE \& J.M. TIEDJE (1985a): Adaptation of denitrifying populations to low soil pH. Appl. Environm. Microbiol. 49, p. 1053-1056.

PAul J.W., V. Etches, and B. J. Zebarth (1997): Increased denitrification below the root zone in the fall following a spring manure application: Canadian Journal of Soil Science, V. 77, p. 249-251.

PAYENE W.J. (1985): Diversity of denitrifiers and their enzymes. In H.L. Goltermann (ed.) Denitrification in the nitrogen cycle, Nato Conference Series I 9, 47-65. 
Pedersen M.B. \& A.M. Lind (1976 a): Nitate reduction in the subsoil I. Introductory studies of the nitrate reduction in the subsoil and its influence on ground water quality. Tidsskr. Planteavl. 80, p. 73-81.

Pedersen M.B. \& A.M. Lind (1976 b): Nitate reduction in the subsoil. IV. Some physical properties of the subsoil, their influence on chemical interchange in the soil, and on ground water quality. Tidsskr. Planteavl. 80, p. 107-118.

Pekdeger A. (1977): Labor- und Felduntersuchungen zur Genese der Sicker- und Grundwasserbeschaffenheit. Diss. Kiel, 228 S.

Postma D. \& B.S., BrockenhuUs-Schack (1987): Diagenesis of iron in proglacial sand deposits of late -and post Weichselian age.- J. Sed. Petrol. 57, p. 1040-1053, Tulsa.

Postma D. (1990): Kinetics of nitrate reduction by detrial Fe(II)-silicates. - Geochim. Cosmochim. Acta 54, p. 903-908.

Postma D., C. Boesen, H. Kristiansen \& F. LARSen (1991): Nitrate reduction in an unconfined aquifer: Water chemistry, reduction processes, and geochemical modelling. Water Res. 27, p. 2027-2045.

REDDY K.R., P.S.C. RAO \& R. E. JESSUP (1982): The effect of Carbon mineralization on denitrification kinetics in mineral and organic soils. Soil Sci. Soc. Am. J. 46, p. $62-68$.

RichteR G.M. \& J. RichTER (1991): Verlagerung und Abbau von Nitrat in der ungesättigten Zone unterhalb des Wurzelrumes. Mitt. Dt. Bodenkdl. Ges. 66 II, S. $1003-1006$.

RoHmann U. \& H. Sontheimer (1985): Nitrat im Grundwasser. Ursachen - Bedeutung - Lösungswege. DVGW-Forschungsstelle am Engler-Bunte- Univ. Karlsruhe.

Rolland W. (1995): Organotrophe und chemolithoautotrophe Denitrifikation in der ungesättigten Zone - Messung und Simulation -. Diss. Techn. Univ. Braunschweig, $124 \mathrm{~S}$.

Rolston D.E., M. Fried \& A. Goldhammer (1976): Denitrification measured directly from nitrogen and nitrous oxide gas fluxes. Soil Sci. Soc. Am. J. 40, p. 259-266.

Rolston D.E., P.S.C., RAO, J.M. DAVIDSON \& R.E. JeSsuP (1984): Simulation of denitrification losses of nitrate fertilizer applied to uncropped, cropped and manureamended field plots. Soil Sci. 137, p. 270-279. 
Rosswall T. (1982): Microbiological regulation of the biogeochemical nitrogen cycle. Plant and Soil 67, p. 15-34.

Ryden J.C., L.J. Lund \& D. D. Focht (1979): Direct measurement of denitrification loss from soil. II. Development and application of field methods. Soil Sci. Soc. Am. J. 43, p. 110-118.

RydEN J. C. (1983): Denitrification loss from a grassland soil in the field receiving different rates of nitrogen as ammonium nitrate. J. Soil Sci. 34, p. 355-365.

SAUERBECK D. \& K. ISERMANN (1993): Herkunft und Strategien zur Verminderung der Emission von klimarelevanten Spurengasen - Bereich Land- und Forstwirtschaft. 7. Mühlheimer wassertechnisches Seminar, IWW-Schriftenreihe, Rhein.-Westfäl. Inst. f. Wasserchemie und Wassertechnol., Mühlheim / Ruhr, S. 1-27.

Scheffer P. \& H. P. Schachtschabel (1992): Lehrbuch der Bodenkunde, 13. Auflage, Enke, Stuttgart.

SCHEFFER P. \& H.P. SCHAChtschaBel (2002): Lehrbuch der Bodenkunde, 15. Auflage, Spektrum Akademischer Verlag Heidelberg. Berlin.

SCHMÄDECKE F. (1998): Lachgas- und Methan-Flüsse eines Gley-Auenbodens unter dem Einfluß einer Raps-Fruchtfolge und in Abhängigkeit von der N-Düngung. Diss. Fachber. Agrarwissenschaften, Univ. Göttingen, 139 S.

SCHMIDER F. \& J.C.G. OTTOW (1984): Die denitrifizierende Mikroflora unterschiedlich belasteter Fließ- und Stillgewässer. Landwirtsch. Forschung 37, S. 181-194.

Scholmayer G., S. Schulte-Kellinghaus \& H. ZePP (1987): Nitrat in der ungesättigten Zone. In: Nitratbelastung des Grundwassers - Beitrag der Landwirtschaft zur Verminderung der Nitratbelastung des Grundwassers. Schriftenreihe des Bundesministers für Landwirt. u. Forsten, Reihe A, 350, S. 127-151.

SChUlte-Kellinghaus S. (1988): Denitrifikation in der ungesättigten Zone mächtiger Lösse und grundwasserferner Standorte. Schriftenreihe d. Bundesmin. f. Ernähr., Landw. u. Forsten. Reihe A: Angew. Wiss. 358, 190 S.

Schlegel H.G. (1985): Allgemeine Mikrobiologie. Thieme Verlag, Stuttgart, New York.

Schlichting E., H.P. Blume. \& K. Stahr (1995): Bodenkundliches Praktikum, Pareys Studientexte 81, Blackwell, Berlin/Wien, 295 S.

SEedorf H.H. \& H.H., Meyer (1992): Landeskunde Niedersachen Band 1, Karl Wachholtz Velag, Neumünster. 
SEILER W. \& R. CONRAD (1981): Mikrobiologische Bildung von $\mathrm{N}_{2} \mathrm{O}$ aus Mineraldünger - ein Umweltproblem? Forum Mikrobiologie 81, S. 223-228.

SiEMENS J. \& M. KAUPENJOHANN (2001): Die Bedeutung der gelösten organischen Substanz (DOM) für die Auswaschung von N und P aus Plaggeneschen. Mitt. Dt. Bodenkdl. Ges. 96 II, S. 455-456

SiEMENS J. (2003): Controls of Carbon, Nitrogen and Phosphours Fluxes in vadose zone and groundwater of protected watersheds in Münster (Germany). Diss. Technische Uni. Berlin, $125 \mathrm{~S}$.

SMITH K.A. (1989): Anaerobic zones and denitrification in soil: modelling and measurement. In: J. Sorensen \& N.P. Revsbech (Eds.), Denitrification in Soil and Sediment. Plenum Press, London.

Sotomayor, D., and C. W. RICE (1996): Denitrification in soil profiles beneath grassland and cultivated soils: Soil Science Society of America Journal, V. 60, p. 18221828.

Souci S.W. (1938): Die Chemie des Moores. F. Enke, Stuttgart, 152 ff.

STAHL B. (1991): Sauersoffzehrung oder „Biochemischer Sauerstoff-Bedarf“ als Maß für die rasch abbaubare organische Substanz von Böden in ihrer Funktion als Substrat der Denitrifikation. Diplomarbeit, Fachb. Agrarwissenschaften, Univ. Göttingen, $76 \mathrm{~S}$.

STANFORD \& SMith (1972): Nitrogen mineralization potentials of soils. Soil Sci. Soc. Am. Proc. 36, p. 465 - 472.

StANFORd G., R. A. VAN DER POL \& S. DzIENIA (1975a): Denitrification rates in relation to total and extractable soil carbon. Soil Sci. Soc. Am. Proc. 39, p. 284-289.

STARR J.L. \& J.Y. PARLANGE (1975): Nonlinear denitrification kinetics with continuous flow in soil columns. Soil Sci. Soc. Am. Proc. 39, p. 875-880.

STREBEL \& RENGER (1978): Vertikale Verlagerung von Nitrat-Stickstoff durch Sickerwasser aus dem wasserungesättigten Boden ins Grundwasser bei Sandböden verschiedener Bodennutzung. Abschlussbericht des DFG-Forschungsvorhabens Str. $163 / 1$ bis 5 .

TEEPE A. (1995): Pleistozäner Schichtaufbau und Relief, Böden, Wasser- und Stickstoffhaushalt der Agrarlandschaft des Vorsfelder Werders bei Wolfsburg. Diplomarbeit, Fachb. Agrarwissenschaften, Univ. Göttingen, 191 S. 
TERRY R.K. \& R.L. TATE (1980): The effect of nitrate on nitrous ocide reduction in organic soils and sediments. Soil Sci. Soc. Am. J. 44, p. 744-746.

TIDJE J.M. (1988): Ecology of denitrification and dissimilatory nitrate reduction to ammonium. In: ZEHNDER, A.J.B. (Hg.): Biology of anaerobic microorganisms. New York. 872 p.

Trudell M.R., R.W. Gillham \& J.A. Cherry (1986): An in-situ study of the occurence and rate of denitrification in a shallow unconfined sand aquifer. J. of Hydrol. 83 , p. 251-268.

Turner F.T. \& W. H. PATriCK jr. (1968): Cemical changes in waterlogged soils as a result of oxygen depletion. Trans. 9. Int. Congr. Soil Sic. Adelaide IV, 53-65.

Van Beek, C. G. E. M; H. Boukes, D. van. Rijsbergen \& R. StraAtman (1988): The threat of the Netherlands waterworks by nitrate in the abstracted groundwater, as demonstrated on the well field Vierlingsbeek. Water Supply 6, 313-318.

VAN Cleemput, O. (1984): Study of nitrogen reduction phenomena in soil. Academie voor Wetenschappen, Letteren en Schone Kunsten van Belgie. Klasse der Wetenschappen 46, p. 55-75.

Vogel, J. C., A. S. Talma \& T. H.E. Heaton (1981): Gaseous nitrogen as evidence for denitrification in groundwater. J. Hydrol. 50, p. 191-200.

VON BEÖCZY E.V. \& B.U. SCHÄFER (1989): Tiefen des Nitratgehaltes in Bodencatenen aus mächtigen Lössdecken mit feuchten Wurzel- und Dränzonen bei hochreichendem Grundwasserkapillarsaum im südlichen Niedersachsen - Prüfung der Hypothese von der Denitrifikationsgunst feuchter Standorte. Diplomarbeit am Institut für Bodenwissenschaften Göttingen, veröffentlicht unter BECKER et al. (1988), Mitt. Dt. Bodenkundl. Ges. 57, S. 147-152.

WARING S.A. \& J.W. GiLliam (1983): The effect of acidity on nitrate reduction and denitrification in rower coastal plain soils. Soil Sci. Soc. Am. J. 47, p. 246 - 251.

Weier K.L., J.W. Gilliam (1993 b): Effect of acidity on denitrification and nitrous oxide evolution from atlantic coastal-plain soils. Soil Sci. Soc. of Am. J. 50, p. 1202-1205.

WeIER K.L., J.W. Doran, J.F Power \& D.T. WALters (1993 a): Denitrification and the dinitrogen/nitrous oxide ratio as affected by soil water, available carbon and nitrate. Soil Biol. Biochem. 25, 8, p. 991-997. 
WeLl R. (1993): Denitrifikation im Wurzelraum unterhalb der Ackerkrume. MessMethodik und Vergleich der ${ }^{15} \mathrm{~N}$-Bilanz mit der ${ }^{15} \mathrm{~N}$-Gasfreisetzungs-Methode. Diss. Univ. Göttingen, $113 \mathrm{~S}$.

Well R. \& D.D. Myrold (1997): Eine neue Methode zur in situ Messung der Denitrifikation in wassergesättigten und ungesättigten Böden. Mitteilungen der Deutschen Bodenkundlichen Gesellschaft 85 II, S. 1063-1066.

Well R., K.W. Becker, R. LANGel \& B. Meyer (1998): Continuous flow equilibration for mass spectrometric analysis of dinitrogen emissions. Soil Sci. Soc. Am. J. 62, p. 906-910.

WELL R. \& D.D. MYROLD (1999): Laboratory evaluation of a new method for in situ measurement of denitrification in watersaturated soils. Soil Biol. Biochem. 31, 81, p. 1109-1119.

Well R., J. Augustin \& K. MeYer (1999): In situ measurement of denitrification and $\mathrm{N}_{2} \mathrm{O}$ production in the saturated zone of three Histosols and of a Gleyic Chernozem. In: G. Broll (Hrsg.): Soil ecological processes in wetlands of Germany.

Well R., H. HöPER \& O. MEhranfar (2000): Field and laboratory studies to quantify actual and long-term denitrification potential in saturated zone of hydromorphic soils of Northwest Germany. Mitt. Dt. Bodenkundl. Ges. 93, S. 108-111.

WeLL R. (2001): Subsoil Denitification. Isotopic tools, in situ methodology, significance for nitrate stability and nitrous oxide turnover in hydromorphic soils under agricultural management. Habilitationsschrift, Göttingen, 139 S.

Well R., J. Augustin \& K. Meyer (2001): In Situ measurement of denitrification and $\mathrm{N}_{2} \mathrm{O}$ production in the saturated zone of three Eutric Histosols and of a Mollic Gleysol. In: G. Broll, W. Merbach \& E.M. Pfeiffer (Eds) Wetlands in central Europe. Soil organisms, soil ecological processes and trace gas emissions, Springer, p. 165-176.

Well R., H. HÖPer \& O. Mehranfar (2001): Empirische Modelle zur Prognose der Denitrifikation im oberflächennahen Grundwasser, Mitt. Dt. Bodenkundl. Ges. 96, S. 667-668.

Well R, Augustin J, Meyer K \& Myrold DD (2003): Comparison of field and laboratory measurement of denitrification and $\mathrm{N}_{2} \mathrm{O}$ production in the saturated zone of hydromorphic soils. Soil Biology and Biochemistry 35, 783 - 799

Wendland F, H. Albert, M. Bach \& R. Schmidt (1993): Atlas zum Nitratstrom in der Bundesrepublik Deutschland. Springer Verlag, Berlin und Heidelberg, 96 S. 
WolfF J., J. Ebeling \& R. NÄVeKe (1985): Abiotische und biotische Stoffumsatzprozesse in pleistozänen Grundwasserkörpern. Z. Dt. geol. Ges. 136, S. 563-574, Hannover. 
11 Anhang 
Anhang 1: Tiefenverteilung der bodenchemischen Parameter sowie der Korngrößenzusammensetzung der untersuchten Auen-Böden. Mittelwert, \pm Standardabweichung, bei Anzahl = 2 Differenzen in Klammern

\begin{tabular}{|c|c|c|c|c|c|c|c|c|c|c|}
\hline \multirow[t]{2}{*}{ Standort } & \multirow[t]{2}{*}{$\begin{array}{l}\text { Tiefe } \\
\mathrm{dm}\end{array}$} & \multirow{2}{*}{$\begin{array}{l}\text { Sulfid-S } \\
\mathrm{mg} / \mathrm{kg}\end{array}$} & \multirow{2}{*}{$\begin{array}{l}\text { C org } \\
\quad \%\end{array}$} & \multirow[t]{2}{*}{$C_{\text {org. }}: \mathrm{N}_{\mathrm{t}}$} & \multirow{2}{*}{$\begin{array}{c}\mathrm{CaCO}_{3} \\
\%\end{array}$} & \multirow[t]{2}{*}{$\begin{array}{l}\text { pH } \\
\left(\mathrm{CaCl}_{2}\right)\end{array}$} & \multirow[t]{2}{*}{ Sand } & \multirow{2}{*}{$\begin{array}{c}\text { Schluff } \\
\%\end{array}$} & \multirow[t]{2}{*}{ Ton } & \multirow[t]{2}{*}{$\mathbf{N}$} \\
\hline & & & & & & & & & & \\
\hline Aher Kämpe & $14-16$ & 39,7 & 2,69 & 10 & 0,00 & 5,7 & 0,3 & 60,5 & 39,2 & 1 \\
\hline Aher Kämpe & $16-18$ & $508 \pm 678$ & $2,7 \pm 0,4$ & $10,1 \pm 0,6$ & $2,4(1,8)$ & 6,6 & $0,42 \pm 0,0$ & $53,2 \pm 0,0$ & $46,4 \pm 0,0$ & 3 \\
\hline Aher Kämpe & $18-20$ & $1140 \pm 1727$ & $1,68 \pm 0,25$ & $10,5 \pm 0,5$ & $6,1(1,7)$ & 7,0 & $0,68 \pm 0,0$ & $60,7 \pm 0,0$ & $38,7 \pm 0,0$ & 3 \\
\hline Aher Kämpe & $20-21$ & $1402 \pm 1425$ & $1,44 \pm 0,75$ & $11,4 \pm 1,0$ & $4,0(2,4)$ & 6,9 & $1,29 \pm 0,0$ & $68,2 \pm 0,0$ & $30,5 \pm 0,0$ & 3 \\
\hline Aher Kämpe & $21-23$ & 709 & 0,87 & 12,2 & 2,9 & 7,2 & 5,8 & 66,8 & 27,4 & 1 \\
\hline Aher Kämpe & $23-25$ & $567(1080)$ & $0,73(1,19)$ & $12,6(0,3)$ & $1,5(0,0)$ & 7,3 & $5,8(0,0)$ & $66,8(0,0)$ & $27,4(0,0)$ & 2 \\
\hline Bühren & $20-22$ & $32,55 \pm 7,73$ & $0,26 \pm 0,11$ & $7,7 \pm 0,8$ & 0,00 & 5,6 & $4,02 \pm 0,4$ & $59,5 \pm 3,0$ & $36,5 \pm 2,9$ & 3 \\
\hline Bühren & $22-24$ & $23,7 \pm 4,6$ & $0,21 \pm 0,03$ & $7,5 \pm 0,6$ & 0,00 & 5,4 & $5,4 \pm 2,78$ & $60,2 \pm 2,6$ & $34,4 \pm 3,6$ & 3 \\
\hline Bühren & $24-26$ & $17,5 \pm 9,6$ & $0,13 \pm 0,02$ & $7,4 \pm 0,4$ & 0,00 & 5,5 & $4,45 \pm 3,9$ & $62,6 \pm 2,6$ & $33,0 \pm 1,3$ & 3 \\
\hline Bühren & $26-28$ & $13,3 \pm 4,1$ & $0,12 \pm 0,04$ & $8,3 \pm 1,9$ & 0,00 & 5,6 & $2,18 \pm 1,6$ & $67,6 \pm 6,3$ & $30,2 \pm 5,9$ & 3 \\
\hline Bühren & $28-30$ & $4,8 \pm 4,6$ & $0,03 \pm 0,01$ & $13,2 \pm 5,9$ & 0,00 & 6,0 & $7,7 \pm 8,4$ & $66,5 \pm 7,2$ & $25,8 \pm 6,4$ & 3 \\
\hline Bühren & $30-32$ & $10,34 \pm 9,04$ & $0,03 \pm 0,01$ & $4,2 \pm 7,3$ & 0,00 & 6,2 & $8,7 \pm 7,4$ & $59 \pm 2,32$ & $32,3 \pm 9,7$ & 3 \\
\hline Reinshof & $10-11$ & $59(11)$ & $0,70(0,56)$ & $8,8(1,4)$ & $0,6(0,6)$ & 7,1 & $11,3(0,0)$ & $46(0,0)$ & $43,1(0,0)$ & 2 \\
\hline Reinshof & $11-12$ & $71(47)$ & $0,53(0,26)$ & $8,7(1,4)$ & $0,6(0,6)$ & 7,1 & $6,3(0,0)$ & $47,0(0,0)$ & $46,7(0,0)$ & 2 \\
\hline Reinshof & $12-13$ & $72(1,4)$ & $0,47(0,17)$ & $8,2(1,8)$ & $1,0(1,0)$ & 6,9 & $7,6(0,0)$ & $56,4(0,0)$ & $36,0(0,0)$ & 2 \\
\hline Reinshof & $13-14$ & 112(31) & $0,48(0,0)$ & $9,2(0,1)$ & $0,4(0,4)$ & 6,9 & $5,2(0,0)$ & $67,7(0,0)$ & $27,0(0,0)$ & 2 \\
\hline Reinshof & $14-15$ & 959(1661) & $2,86(4,66)$ & $14,7(6,6)$ & $6,69(12)$ & 6,9 & $8,5(0,0)$ & $68,4(0,0)$ & $23,1(0,0)$ & 2 \\
\hline Reinshof & $15-16$ & 781(1405) & $1,44(1,93)$ & $14,1(6,3)$ & $3,7(7,4)$ & 6,9 & $23,9(0,0)$ & $60,0(0,0)$ & $16,1(0,0)$ & 2 \\
\hline Reinshof & $16-17$ & $1915(1254)$ & $2,09(2,48)$ & $18,8(8,5)$ & $6(11)$ & 6,7 & $41,5(0,0)$ & $49,9(0,0)$ & $8,6(0,0)$ & 2 \\
\hline Reinshof & $17-18$ & 1908(82) & $2,79(1,02)$ & $17,8(3,4)$ & $5,1(9,8)$ & 6,6 & $32,7(0,0)$ & $55(0,0)$ & $12,3(0,0)$ & 2 \\
\hline Reinshof & $18-19$ & $6230(6880)$ & $11,5(12,6)$ & $22,6(8,5)$ & $4,6(9,1)$ & 6,2 & $32,7(0,0)$ & $55(0,0)$ & $12,3(0,0)$ & 2 \\
\hline Reinshof & $19-20$ & $9580(18615)$ & $15,7(30,4)$ & $14,2(8,6)$ & $0,4(0,1)$ & 7,1 & $32,7(0,0)$ & $55(0,0)$ & $12,3(0,0)$ & 2 \\
\hline Schladen & $16-18$ & $20,37(4,02)$ & $0,46(0,09)$ & $8,6(0,5)$ & 0,6 & 7,0 & $6,68(0,0)$ & $76,9(0,0)$ & $16,5(0,0)$ & 2 \\
\hline Schladen & $18-20$ & $21,95 \pm 9,94$ & $0,92 \pm 0,64$ & $10,6 \pm 1,4$ & $0,2 \pm 0,4$ & 6,3 & $13,0 \pm 0,0$ & $74,2 \pm 0,0$ & $12,8 \pm 0,0$ & 3 \\
\hline Schladen & $20-22$ & $35,1 \pm 18,1$ & $1,77 \pm 0,73$ & $14,0 \pm 2,7$ & $0,1 \pm 0,2$ & 5,9 & $20,4 \pm 0,0$ & $66,5 \pm 0,0$ & $13,1 \pm 0,0$ & 3 \\
\hline Schladen & $22-24$ & $199 \pm 207$ & $3,32 \pm 1,29$ & $16,6 \pm 4,2$ & 0,00 & 5,4 & $26,9 \pm 0,0$ & $54,8 \pm 0,0$ & $18,4 \pm 0,0$ & 3 \\
\hline Schladen & $24-26$ & $292 \pm 207$ & $3,98 \pm 1,26$ & $20,6 \pm 3,2$ & 0,00 & 5,2 & $46,9 \pm 0,0$ & $41,0 \pm 0,0$ & $12,2 \pm 0,0$ & 3 \\
\hline Schladen & $26-28$ & $450 \pm 140$ & $2,76 \pm 0,96$ & $19,7 \pm 0,5$ & 0,00 & 5,3 & $52,5 \pm 0,0$ & $24,7 \pm 0,0$ & $22,8 \pm 0,0$ & 3 \\
\hline Schladen & $28-30$ & $840 \pm 1029$ & $2,01 \pm 1,38$ & $19,1 \pm 2,6$ & 0,00 & 3,2 & $53,3 \pm 0,0$ & $25,4 \pm 0,0$ & $21,3 \pm 0,0$ & 3 \\
\hline Schladen & $30-32$ & 1448(183) & $2,94(1,08)$ & $20,8(2,3)$ & 0,00 & 4,8 & $53(28)$ & $25(14)$ & $21(14)$ & 2 \\
\hline Schladen & $32-34$ & $320(92)$ & $1,23(0,77)$ & $18,2(0,5)$ & 0,00 & 5,1 & $53(28)$ & $25(14)$ & $21(14)$ & 2 \\
\hline
\end{tabular}


Anhang 2: Tiefenverteilung der chemische Eigenschaften und Korngrößenzusammensetzung der untersuchten Sand-Böden. Mittelwert, \pm Standardabweichung, bei Anzahl = 2 Differenzen in Klammern

\begin{tabular}{|c|c|c|c|c|c|c|c|c|c|c|}
\hline \multirow[t]{2}{*}{ Standort } & \multirow[t]{2}{*}{$\begin{array}{l}\text { Tiefe } \\
(\mathrm{dm})\end{array}$} & \multirow[t]{2}{*}{$\begin{array}{l}\text { Sulfid-S } \\
\mathrm{mg} / \mathrm{kg}\end{array}$} & \multirow{2}{*}{$\begin{array}{l}\text { C }_{\text {org. }} \\
\quad \%\end{array}$} & \multirow[t]{2}{*}{$C_{\text {org: }}: N_{t}$} & \multirow[t]{2}{*}{$\begin{array}{l}\mathrm{CaCO}_{3} \\
\mathrm{mg} / \mathrm{Kg}\end{array}$} & \multirow[t]{2}{*}{$\begin{array}{l}\mathrm{pH} \\
\left(\mathrm{CaCl}_{2}\right)\end{array}$} & \multirow[t]{2}{*}{ Sand } & \multirow{2}{*}{$\frac{\text { Schluff }}{\%}$} & \multirow[t]{2}{*}{ Ton } & \multirow[t]{2}{*}{$\mathbf{N}$} \\
\hline & & & & & & & & & & \\
\hline Dinklage & $12-14$ & $2,52(3,23)$ & $0,15(0,02)$ & $27,3(15,9)$ & 0,00 & 4,3 & $91,6(0,0)$ & $7,6(0,0)$ & $0,79(0,0)$ & 2 \\
\hline Dinklage & $14-16$ & $2,1 \pm 1,2$ & $0,13 \pm 0,06$ & $27 \pm 12$ & 0,00 & 4,4 & $71 \pm 18$ & $25,8 \pm 15,8$ & $3,36 \pm 2,23$ & 3 \\
\hline Dinklage & $16-18$ & $4,42 \pm 1,64$ & $0,17 \pm 0,04$ & $18,9 \pm 2,2$ & 0,00 & 4,5 & $75,7 \pm 13,3$ & $21,5 \pm 11,6$ & $2,74 \pm 1,65$ & 3 \\
\hline Dinklage & $18-20$ & $3,8 \pm 1,3$ & $0,1 \pm 0,03$ & $26,6 \pm 4,54$ & 0,00 & 4,6 & $88,3 \pm 4,3$ & $10,5 \pm 3,8$ & $1,23 \pm 0,49$ & 3 \\
\hline Dinklage & $20-22$ & $2,85 \pm 0,97$ & $0,1 \pm 0,04$ & $23,2 \pm 3,6$ & 0,00 & 4,6 & $74,8 \pm 13,8$ & $23,2 \pm 12,9$ & $1,99 \pm 0,90$ & 3 \\
\hline Dinklage & $22-24$ & $8,7 \pm 3,5$ & $0,14 \pm 0,1$ & $19,0 \pm 3,2$ & 0,00 & 4,6 & $73 \pm 7$ & $24,9 \pm 6,5$ & $1,75 \pm 0,65$ & 3 \\
\hline Dinklage & $24-26$ & $2,11(2,17)$ & $0,14(0,1)$ & $19,9(20,9)$ & 0,00 & 5,7 & $76,6(3,4)$ & $22,0(2,5)$ & $1,38(0,85)$ & 2 \\
\hline Dinklage & $26-28$ & $6,03 \pm 4,23$ & $0,13 \pm 0,1$ & $32,6 \pm 10,4$ & 0,00 & 4,9 & $79,6 \pm 1,9$ & $18,9 \pm 1,3$ & $1,57 \pm 0,62$ & 3 \\
\hline Dinklage & $28-30$ & $28,5 \pm 10,4$ & $0,17 \pm 0,06$ & $26,6 \pm 10,5$ & 0,00 & 4,8 & $79,0 \pm 2,6$ & $19,4 \pm 1,4$ & $1,66 \pm 0,85$ & 3 \\
\hline Dinklage & $30-32$ & 35,4 & 0,19 & 19,9 & 0,00 & 4,5 & 75,7 & 23,0 & 1,3 & 1 \\
\hline Dinklage & $32-34$ & 28,1 & 0,18 & 17,3 & 0,00 & 4,5 & 69,4 & 29,3 & 1,3 & 1 \\
\hline Dinklage & $34-36$ & 12,1 & 0,14 & 15,7 & 0,00 & 4,3 & 67,5 & 30,8 & 1,7 & 1 \\
\hline Dinklage & $36-38$ & 18,3 & 0,17 & 15,2 & 0,00 & 4,3 & 67,0 & 31,2 & 1,8 & 1 \\
\hline Dinklage & $38-40$ & 27,2 & 0,18 & 14,1 & 0,00 & 4,4 & 70,2 & 28,4 & 1,4 & 1 \\
\hline Freistatt & $14-16$ & 0,54 & 0,25 & 39,2 & 0,00 & 4,5 & n. b. & n. b. & n. b. & 1 \\
\hline Freistatt & $16-18$ & 0,00 & 0,18 & 45,1 & 0,00 & 4,5 & n. b. & n. b. & n. b. & 1 \\
\hline Freistatt & $20-22$ & 4,76 & 0,16 & 46,0 & 0,00 & 4,5 & n. b. & n. b. & n. b. & 1 \\
\hline Freistatt & $22-25$ & 6,66 & 0,17 & 49,1 & 0,00 & 4,3 & n. b. & n. b. & n. b. & 1 \\
\hline Freistatt & $25-27$ & 8,72 & 0,15 & 43,7 & 0,00 & 4,3 & n. b. & n. b. & n. b. & 1 \\
\hline Freistatt & $27-29$ & 0,00 & 0,14 & 31,6 & 0,00 & 4,3 & n. b. & n. b. & n. b. & 1 \\
\hline Freistatt & $29-30$ & 6,58 & 0,17 & 41,5 & 0,00 & 4,4 & n. b. & n. b. & n. b. & 1 \\
\hline Freistatt & $16-18$ & 4,07 & 0,30 & 42,7 & 0,00 & 4,3 & n. b. & n. b. & n. b. & 1 \\
\hline Freistatt & $18-20$ & 7,11 & 0,20 & 44,9 & 0,00 & 4,3 & n. b. & n. b. & n. b. & 1 \\
\hline Freistatt & $20-22$ & 4,98 & 0,17 & 31,2 & 0,00 & 4,2 & n. b. & n. b. & n. b. & 1 \\
\hline Freistatt & $22-24$ & 4,76 & 0,09 & 20,9 & 0,00 & 4,5 & n. b. & n. b. & n. b. & 1 \\
\hline Freistatt & $24-26$ & 2,44 & 0,09 & 27,0 & 0,00 & 4,5 & n. b. & n. b. & n. b. & 1 \\
\hline Freistatt & $26-28$ & 4,88 & 0,03 & 19,7 & 0,00 & 4,5 & n. b. & n. b. & n. b. & 1 \\
\hline Freistatt & $28-30$ & 2,77 & 0,05 & 21,5 & 0,00 & 4,6 & n. b. & n. b. & n. b. & 1 \\
\hline Freistatt & $16-18$ & 4,78 & 0,07 & 8,56 & 0,00 & 4,1 & n. b. & n. b. & n. b. & 1 \\
\hline Freistatt & $18-20$ & 7,22 & 0,04 & 6,85 & 0,00 & 4,0 & n. b. & n. b. & n. b. & 1 \\
\hline Freistatt & $20-22$ & 7,58 & 0,06 & 9,31 & 0,00 & 4,2 & n. b. & n. b. & n. b. & 1 \\
\hline Freistatt & $22-24$ & 6,40 & 0,09 & 11,1 & 0,00 & 4,3 & n. b. & n. b. & n. b. & 1 \\
\hline Freistatt & $24-26$ & 10,8 & 0,07 & 9,86 & 0,00 & 4,2 & n. b. & n. b. & n. b. & 1 \\
\hline Freistatt & $26-28$ & 1,98 & 0,03 & 8,38 & 0,00 & 4,2 & n. b. & n. b. & n. b. & 1 \\
\hline Freistatt & $28-30$ & 4,08 & 0,06 & 12,3 & 0,00 & 4,2 & n. b. & n. b. & n. b. & 1 \\
\hline Fuhrberg & $10-12$ & 5,38 & 0,34 & 27,5 & 0,00 & 5,5 & 0,00 & 0,00 & 0,00 & 1 \\
\hline Fuhrberg & $12-14$ & 0,00 & 0,12 & 26,4 & 0,00 & 4,9 & 0,00 & 0,00 & 0,00 & 1 \\
\hline Fuhrberg & $14-16$ & 1,24 & 0,05 & 14,0 & 0,00 & 5,1 & 0,00 & 0,00 & 0,00 & 1 \\
\hline Fuhrberg & $16-18$ & 0,00 & 0,06 & 13,0 & 0,00 & 5,1 & 97,0 & 0,1 & 2,9 & 1 \\
\hline Fuhrberg & $18-20$ & 0,00 & 0,06 & 13,9 & 0,00 & 5,2 & 98,2 & 0,1 & 1,7 & 1 \\
\hline Fuhrberg & $20-22$ & 1,14 & 0,06 & 21,2 & 0,00 & 5,1 & 99,2 & 0,2 & 0,6 & 1 \\
\hline Fuhrberg & $22-24$ & 0,77 & 0,06 & 12,8 & 0,00 & 5,3 & 98,7 & 0,2 & 1,2 & 1 \\
\hline Fuhrberg & $24-26$ & 0,00 & 0,10 & 20,7 & 0,00 & 4,8 & 98,5 & 0,1 & 1,4 & 1 \\
\hline
\end{tabular}


Anhang 2 (Fortsetzung): Tiefenverteilung der bodenchemischen Parameter sowie der Korngrößenzusammensetzung der untersuchten Sand-Böden. Mittelwert, \pm Standardabweichung,

bei Anzahl=2 Differenzen in Klammern

\begin{tabular}{|c|c|c|c|c|c|c|c|c|c|c|}
\hline \multirow[t]{2}{*}{ Standort } & \multirow[t]{2}{*}{$\begin{array}{l}\text { Tiefe } \\
(\mathrm{cm})\end{array}$} & \multirow[t]{2}{*}{$\begin{array}{l}\text { Sulfid-S } \\
\mathrm{mg} / \mathrm{kg}\end{array}$} & \multirow{2}{*}{$\begin{array}{l}\text { C }_{\text {org }} \\
\quad \%\end{array}$} & \multirow[t]{2}{*}{$C_{\text {org }}: \mathbf{N}_{t}$} & \multirow[t]{2}{*}{$\begin{array}{l}\mathrm{CaCO}_{3} \\
\mathrm{mg} / \mathrm{Kg}\end{array}$} & \multirow[t]{2}{*}{$\begin{array}{l}\mathrm{pH} \\
\left(\mathrm{CaCl}_{2}\right)\end{array}$} & \multirow[t]{2}{*}{ Sand } & \multirow{2}{*}{$\begin{array}{c}\text { Schluff } \\
\%\end{array}$} & \multirow[t]{2}{*}{ Ton } & \multirow[t]{2}{*}{$\mathbf{N}$} \\
\hline & & & & & & & & & & \\
\hline Fuhrberg & $26-28$ & 0,00 & 0,13 & 23,8 & 0,00 & 5,0 & 98,2 & 0,2 & 1,6 & 1 \\
\hline Fuhrberg & $28-30$ & 0,00 & 0,08 & 23,3 & 0,00 & 4,7 & 99,1 & 0,2 & 0,7 & 1 \\
\hline Fuhrberg & $30-32$ & 1,58 & 0,12 & 30,3 & 0,00 & 4,8 & 98,3 & 0,1 & 1,6 & 1 \\
\hline Fuhrberg & $32-34$ & 1,86 & 0,11 & 30,1 & 0,00 & 4,9 & 98,9 & 0,1 & 1,0 & 1 \\
\hline Fuhrberg & $34-36$ & 0,00 & 0,08 & 26,5 & 0,00 & 5,1 & 99,1 & 0,1 & 0,8 & 1 \\
\hline Fuhrberg & $36-38$ & 0,00 & 0,06 & 14,5 & 0,00 & 5,2 & 95,5 & 0,2 & 4,3 & 1 \\
\hline Markhausen & $15-17$ & $45,9 \pm 55$ & $0,63 \pm 0,58$ & $47,6 \pm 0,6$ & $0 \pm 0$ & 4,5 & $95,3 \pm 0,0$ & $1,96 \pm 0,0$ & $2,7 \pm 0,0$ & 3 \\
\hline Markhausen & $17-19$ & $145 \pm 229$ & $1,38 \pm 1,95$ & $42,5 \pm 20,9$ & $0 \pm 0$ & 4,4 & $95,3 \pm 0,0$ & $1,96 \pm 0,0$ & $2,7 \pm 0,0$ & 3 \\
\hline Markhausen & $19-21$ & $77,7 \pm 81,3$ & $0,62 \pm 0,52$ & $40,2 \pm 20,3$ & $0 \pm 0$ & 4,5 & $95,3 \pm 0,0$ & $1,96 \pm 0,0$ & $2,7 \pm 0,0$ & 3 \\
\hline Markhausen & $21-23$ & $53,5 \pm 65,2$ & $0,52 \pm 0,3$ & $71,9 \pm 33,5$ & $0 \pm 0$ & 4,6 & $95,3 \pm 0,0$ & $1,96 \pm 0,0$ & $2,7 \pm 0,0$ & 3 \\
\hline Markhausen & $23-25$ & $49,3 \pm 69,3$ & $0,31 \pm 0,34$ & $44,0 \pm 22,0$ & $0 \pm 0$ & 4,7 & $95,3 \pm 0,0$ & $1,96 \pm 0,0$ & $2,7 \pm 0,0$ & 3 \\
\hline Markhausen & $25-27$ & $29,4 \pm 26,9$ & $0,16 \pm 0,12$ & $36,7 \pm 24,7$ & $0 \pm 0$ & 4,8 & $95,3 \pm 0,0$ & $1,96 \pm 0,0$ & $2,7 \pm 0,0$ & 3 \\
\hline Markhausen & $27-29$ & $37,8 \pm 25,4$ & $0,11 \pm 0,03$ & $25,3 \pm 2,1$ & $0 \pm 0$ & 4,6 & $95,3 \pm 0,0$ & $1,96 \pm 0,0$ & $2,7 \pm 0$ & 3 \\
\hline Markhausen & $29-31$ & $82,8(155)$ & $0,27(0,41)$ & $54,6(65,4)$ & $0(0)$ & 4,6 & $95,3(0,0)$ & $1,96(0,0)$ & $2,7(0,0)$ & 2 \\
\hline Oldendorf & $10-12$ & $63,9 \pm 51,5$ & $0,16 \pm 0,07$ & $38,8 \pm 17,8$ & $0 \pm 0$ & 5,5 & $93,1 \pm 0,0$ & $5,9 \pm 0,0$ & $1,03 \pm 0,0$ & 7 \\
\hline Oldendorf & $12-14$ & $104,5 \pm 67,2$ & $0,19 \pm 0,11$ & $28,2 \pm 7,5$ & $0,03 \pm 0,1$ & 5,4 & $93,1 \pm 0,0$ & $5,9 \pm 0,0$ & $1,03 \pm 0,0$ & 7 \\
\hline Oldendorf & $14-16$ & $119,8 \pm 84,8$ & $0,18 \pm 0,11$ & $31,0 \pm 13,1$ & $0,1 \pm 0,2$ & 5,5 & $93,1 \pm 0,0$ & $5,9 \pm 0,0$ & $1,03 \pm 0,0$ & 6 \\
\hline Vechtel & $11-12$ & $3,83 \pm 1,19$ & $0,10 \pm 0,01$ & $24,2 \pm 3,0$ & $0(0)$ & 5,2 & $93,1 \pm 0,0$ & $5,9 \pm 0,0$ & $1,03 \pm 0,0$ & 3 \\
\hline Vechtel & $12-13$ & $4,08 \pm 1,21$ & $0,08 \pm 0,02$ & $36,3 \pm 13,6$ & $0(0)$ & 5,2 & $93,1 \pm 0,0$ & $5,9 \pm 0,0$ & $1,03 \pm 0,0$ & 3 \\
\hline Vechtel & $13-14$ & $2,93 \pm 2,08$ & $0,15 \pm 0,09$ & $32,5 \pm 13,9$ & $0(0)$ & 4,9 & $88,7 \pm 7,6$ & $8,9 \pm 5,3$ & $2,4 \pm 2,3$ & 3 \\
\hline Vechtel & $8-9$ & $1,99(3,98)$ & $0,12(0,0)$ & $43,8(32,9)$ & $0(0)$ & 5,1 & $93,1(0,0)$ & $5,9(0,0)$ & $1,03(0,0)$ & 2 \\
\hline Vechtel & $9-10$ & $3,52(0,35)$ & $0,11(0,03)$ & $28,2(23,6)$ & $0(0)$ & 5,1 & $93,1(0,0)$ & $5,9(0,0)$ & $1,03(0,0)$ & 2 \\
\hline Vechtel & $10-11$ & $4,12(0,09)$ & $0,09(0,01)$ & $23,7(6,0)$ & $0(0)$ & 5,1 & $93,1(0,0)$ & $5,9(0,0)$ & $1,03(0,0)$ & 2 \\
\hline
\end{tabular}


Anhang 3: Tiefenverteilung der bodenchemischen Parameter sowie der Korngrößenzusammensetzung der untersuchten Seemarsch. Mittelwert, \pm Standardabweichung, bei Anzahl = 2 Differenzen in Klammern

\begin{tabular}{|c|c|c|c|c|c|c|c|c|c|c|}
\hline \multirow[t]{2}{*}{ Standort } & \multirow[t]{2}{*}{$\begin{array}{l}\text { Tiefe } \\
(\mathrm{dm})\end{array}$} & \multirow[t]{2}{*}{$\begin{array}{l}\text { Sulfid-S } \\
\mathrm{mg} / \mathrm{kg}\end{array}$} & \multirow{2}{*}{$\begin{aligned} \text { C }_{\text {org }} & \\
& \%\end{aligned}$} & \multirow[t]{2}{*}{$C_{\text {org }}: \mathrm{N}_{\mathrm{t}}$} & \multirow[t]{2}{*}{$\underset{\%}{\mathrm{CaCO}_{3}}$} & \multirow[t]{2}{*}{$\begin{array}{l}\mathrm{pH} \\
\left(\mathrm{CaCl}_{2}\right)\end{array}$} & \multirow[t]{2}{*}{ Sand } & \multirow{2}{*}{$\begin{array}{c}\text { Schluff } \\
\%\end{array}$} & \multirow[t]{2}{*}{ Ton } & \multirow[t]{2}{*}{$\mathbf{N}$} \\
\hline & & & & & & & & & & \\
\hline $\begin{array}{l}\text { Neuhäuser- } \\
\text { Felde }\end{array}$ & $10-12$ & $11,1 \pm 6,3$ & $0,41 \pm 0,23$ & $13,4 \pm 6,9$ & $0,24 \pm 0,42$ & 6,2 & $49,1 \pm 0,0$ & $37,8 \pm 0,0$ & $13,1 \pm 0,0$ & 3 \\
\hline $\begin{array}{l}\text { Neuhäuser- } \\
\text { Felde }\end{array}$ & $12-14$ & 0,00 & $0,64 \pm 0,28$ & $10,8 \pm 8,3$ & $0,06 \pm 0,1$ & 6,2 & $30,3 \pm 0,0$ & $50,7 \pm 0,0$ & $19,0 \pm 0,0$ & 3 \\
\hline $\begin{array}{l}\text { Neuhäuser- } \\
\text { Felde }\end{array}$ & 14-16 & 0,00 & $0,37 \pm 0,06$ & $9,1 \pm 0,9$ & 0,00 & 6,2 & $7,5 \pm 3,6$ & $59,5 \pm 0,1$ & $33,0 \pm 3,7$ & 3 \\
\hline $\begin{array}{l}\text { Neuhäuser- } \\
\text { Felde }\end{array}$ & $16-18$ & 0,00 & $0,25 \pm 0,01$ & $9,1 \pm 0,8$ & 0,00 & 6,3 & $17,9 \pm 0,0$ & $55,9 \pm 0,0$ & $26,2 \pm 0,0$ & 3 \\
\hline $\begin{array}{l}\text { Neuhäuser- } \\
\text { Felde }\end{array}$ & $18-20$ & 0,00 & $0,19 \pm 0,01$ & $9,3 \pm 0,5$ & 0,00 & 6,3 & $34,3 \pm 0,0$ & $54,9 \pm 0,0$ & $10,9 \pm 0,0$ & 3 \\
\hline $\begin{array}{l}\text { Neuhäuser- } \\
\text { Felde }\end{array}$ & $20-22$ & $6,15 \pm 0,78$ & $0,13 \pm 0,13$ & $6,5 \pm 5,8$ & $1,43 \pm 1,28$ & 6,8 & $27, \pm 0,0$ & $57,2 \pm 0,0$ & $15,3 \pm 0,0$ & 3 \\
\hline $\begin{array}{l}\text { Neuhäuser- } \\
\text { Felde }\end{array}$ & $22-24$ & $4,58 \pm 3,96$ & $0,11 \pm 0,01$ & $8,2 \pm 1,2$ & $4,77 \pm 0,18$ & 7,2 & $33,2 \pm 0,0$ & $62,5 \pm 0$ & $4,3 \pm 0,0$ & 3 \\
\hline $\begin{array}{l}\text { Neuhäuser- } \\
\text { Felde }\end{array}$ & $24-26$ & $35,6 \pm 10,5$ & $0,30 \pm 0,03$ & $14,3 \pm 2,9$ & $5,15 \pm 0,35$ & 7,1 & $22,6 \pm 0,0$ & $57,9 \pm 0,0$ & $19,6 \pm 0,0$ & 3 \\
\hline $\begin{array}{l}\text { Neuhäuser- } \\
\text { Felde }\end{array}$ & $26-28$ & $417 \pm 293$ & $0,41 \pm 0,12$ & $9,41 \pm 0,49$ & $6,43 \pm 0,71$ & 6,9 & $13,1 \pm 0,0$ & $68,9 \pm 0,0$ & $18,0 \pm 0,0$ & 3 \\
\hline $\begin{array}{l}\text { Neuhäuser- } \\
\text { Felde }\end{array}$ & $28-30$ & $756 \pm 562$ & $0,54 \pm 0,14$ & $11,1 \pm 0,3$ & $6,63 \pm 0,32$ & 6,8 & $20,5 \pm 0,0$ & $68,9 \pm 0,0$ & $10,6 \pm 0,0$ & 3 \\
\hline $\begin{array}{l}\text { Neuhäuser- } \\
\text { Felde }\end{array}$ & $30-32$ & $1081 \pm 235$ & $0,63 \pm 0,14$ & $11,8 \pm 3,2$ & $6,42 \pm 1,04$ & 6,6 & $40,1 \pm 0,0$ & $51,9 \pm 0,0$ & $8,03 \pm 0,0$ & 3 \\
\hline $\begin{array}{l}\text { Neuhäuser- } \\
\text { Felde }\end{array}$ & $32-34$ & $1559(760)$ & $0,73(0,08)$ & $11,9(0,1)$ & $6,7(0,8)$ & 6,7 & $47,4(0,0)$ & $42,2(0,0)$ & $10,5(0,0)$ & 2 \\
\hline $\begin{array}{l}\text { Neuhäuser- } \\
\text { Felde }\end{array}$ & $34-36$ & 2213(889) & $0,84(0,43)$ & $11,4(0,7)$ & $7,3(0,8)$ & 6,6 & $31,2(0,0)$ & $51,7(0,0)$ & $17,1(0,0)$ & 2 \\
\hline $\begin{array}{l}\text { Neuhäuser- } \\
\text { Felde }\end{array}$ & $36-38$ & 898 & 0,5 & 15,6 & 7,6 & 6,7 & 48,0 & 45,2 & 6,7 & 1 \\
\hline $\begin{array}{l}\text { Neuhäuser- } \\
\text { Felde }\end{array}$ & $38-40$ & 983 & 0,4 & 11,6 & 7,9 & 6,7 & 57,3 & 38,0 & 4,7 & 1 \\
\hline
\end{tabular}


Anhang 4: Zusammenfassung der Ergebnisse zur Reduktion von Nitrat und Produktion von Sulfat aus den anaeroben und aeroben Inkubationsversuchen: Schichtmittelwerte der Umsatzraten zu Versuchsbeginn $\left(\mathrm{R}_{\mathrm{NO} 3}, \mathrm{DR}^{15}\left(\mathrm{~N}_{2}+\mathbf{N}_{2} \mathrm{O}\right)\right.$, $\left.\mathbf{R}_{\mathrm{SO} 4}\right)$, kumulierte Umsetzungen (MO3, $\left.M_{\text {SO4 }}\right)$, Anteil der heterotrophen Denitrifikation am Gesamtumsatz $\left(M_{\mathrm{NO} 3} \mathrm{H}: \mathrm{M}_{\mathrm{NO} 3(g e s)}\right)$, Nachhaltigkeits- und Verfügbarkeits-Indizies (NI, VI)

\begin{tabular}{|c|c|c|c|c|c|c|c|c|c|c|c|c|c|c|c|c|c|c|c|c|c|c|}
\hline Standort & $\begin{array}{l}\text { Schicht- } \\
\text { folge } \\
\text { [dm] }\end{array}$ & $\mathbf{R}_{\mathrm{NO} 3}$ & $\begin{array}{l}\text { DR } \\
{ }^{15}\left(\mathrm{~N}_{2}+\mathrm{N}_{2} \mathrm{O}\right)\end{array}$ & $\begin{array}{l}M_{\mathrm{NO} 3} \\
500 d\end{array}$ & $\begin{array}{l}\mathrm{M}_{\mathrm{NO} 3} \\
(\infty)\end{array}$ & $\begin{array}{l}\mathrm{NI}_{\mathrm{NO} 3} \\
500 \mathrm{~d}\end{array}$ & $\begin{array}{l}\mathrm{VI}_{\mathrm{NO} 3} \\
500 \mathrm{~d}\end{array}$ & $\begin{array}{l}\mathrm{VI}_{\mathrm{NO} 3} \\
(\infty)\end{array}$ & $\begin{array}{l}\mathbf{M}_{\mathrm{NO3}} \\
\mathbf{5 0 0 d} \\
\mathbf{H}\end{array}$ & $\begin{array}{l}\mathrm{VI}_{\mathrm{NO3}} \\
500 \mathrm{~d} \\
\mathrm{H}\end{array}$ & $\begin{array}{l}\mathrm{M}_{\mathrm{NO} 3} \mathrm{H}: \\
\mathrm{M}_{\mathrm{NO} 3 \mathrm{ges}} \\
500 \mathrm{~d}\end{array}$ & $\mathbf{R}_{\mathrm{SO} 4}$ & $\begin{array}{l}M_{\text {SO4 }} \\
\text { 500d }\end{array}$ & $\begin{array}{l}M_{\text {SO4 }} \\
(\infty)\end{array}$ & $\begin{array}{l}\mathrm{NI}_{\mathrm{SO} 4} \\
\mathbf{5 0 0 \mathrm { d }}\end{array}$ & $\begin{array}{l}\mathrm{VI}_{\mathrm{SO} 4} \\
\mathbf{5 0 0 d}\end{array}$ & $\begin{array}{l}\mathrm{VI}_{\mathrm{SO} 4} \\
(\infty)\end{array}$ & $\begin{array}{l}M_{\text {SO4 }} \\
500 d \\
A\end{array}$ & $\begin{array}{l}M_{\text {SO4 }} \\
(\infty) \\
\text { A }\end{array}$ & $\begin{array}{l}\mathrm{NISO4}_{\mathrm{SO}} \\
500 \mathrm{~d} \\
\mathrm{~A}\end{array}$ & $\begin{array}{l}\mathrm{VI}_{\mathrm{SO} 4} \\
\mathbf{5 0 0 d} \\
\mathrm{A}\end{array}$ & $\mathbf{N}$ \\
\hline $\begin{array}{l}\text { Aher- } \\
\text { kämpe }\end{array}$ & $14-21$ & $\begin{array}{l}1,8 \\
\pm 0,74\end{array}$ & $\begin{array}{l}1,56 \\
\pm 1,15\end{array}$ & $\begin{array}{l}450 \\
\pm 134\end{array}$ & $\begin{array}{l}1327 \\
\pm 311\end{array}$ & $\begin{array}{l}0,45 \\
\pm 0,26\end{array}$ & $\begin{array}{l}0,03 \\
\pm 0,01\end{array}$ & $\begin{array}{l}0,08 \\
\pm 0,06\end{array}$ & $\begin{array}{l}336 \\
\pm 178\end{array}$ & $\begin{array}{l}0,02 \\
\pm 0,01\end{array}$ & $\begin{array}{l}0,71 \\
\pm 0,29\end{array}$ & $\begin{array}{l}0,85 \\
\pm 0,7\end{array}$ & $\begin{array}{l}172 \\
\pm 128\end{array}$ & $\begin{array}{l}98 \\
\pm 85\end{array}$ & $\begin{array}{l}0,33 \\
\pm 0,29\end{array}$ & $\begin{array}{l}0,55 \\
\pm 0,56\end{array}$ & $\begin{array}{l}0,33 \\
\pm 0,36\end{array}$ & $\begin{array}{l}341 \\
\pm 278\end{array}$ & $\begin{array}{l}3141 \\
\pm 2534\end{array}$ & $\begin{array}{l}0,26 \\
\pm 0,14\end{array}$ & $\begin{array}{l}0,76 \\
\pm 0,38\end{array}$ & 10 \\
\hline $\begin{array}{l}\text { Aher- } \\
\text { Kämpe }\end{array}$ & $21-24$ & $\begin{array}{l}0,75 \\
\pm 0,51\end{array}$ & $\begin{array}{l}0,63 \\
\pm 0,8\end{array}$ & $\begin{array}{l}295 \\
\pm 263\end{array}$ & $\begin{array}{l}353 \\
\pm 316\end{array}$ & $\begin{array}{l}0,23 \\
\pm 0,25\end{array}$ & $\begin{array}{l}0,03 \\
\pm 0,02\end{array}$ & $\begin{array}{l}0,03 \\
\pm 0,03\end{array}$ & $\begin{array}{l}158 \\
\pm 145\end{array}$ & $\begin{array}{l}0,02 \\
\pm 0,01\end{array}$ & $\begin{array}{l}0,35 \\
\pm 0,31\end{array}$ & $\begin{array}{l}0,81 \\
\pm 0,77\end{array}$ & $\begin{array}{l}219 \\
\pm 152\end{array}$ & $\begin{array}{l}131 \\
\pm 112\end{array}$ & $\begin{array}{l}0,46 \\
\pm 0,21\end{array}$ & $\begin{array}{l}0,23 \\
\pm 0,21\end{array}$ & $\begin{array}{l}0,20 \\
\pm 0,03\end{array}$ & $\begin{array}{l}557 \\
\pm 150\end{array}$ & $\begin{array}{l}1055 \\
\pm 660\end{array}$ & $\begin{array}{l}0,8 \\
\pm 0,46\end{array}$ & $\begin{array}{l}0,5 \\
\pm 0,5\end{array}$ & 3 \\
\hline Bühren & $20-26$ & $\begin{array}{l}0,16 \\
\pm 0,37\end{array}$ & $\begin{array}{l}0,10 \\
\pm 0,09\end{array}$ & $\begin{array}{l}42 \\
\pm 33\end{array}$ & $\begin{array}{l}20 \\
\pm 24\end{array}$ & $\pm 0,0$ & $\begin{array}{l}0,02 \\
\pm 0,02\end{array}$ & $\begin{array}{l}0,01 \\
\pm 0,02\end{array}$ & $\begin{array}{l}42 \\
\pm 33\end{array}$ & $\begin{array}{l}0,03 \\
\pm 0,02\end{array}$ & $\begin{array}{l}1,0 \\
\pm 0,0\end{array}$ & $\begin{array}{l}02 \\
0,02\end{array}$ & $\begin{array}{l}6,0 \\
\pm 5,0\end{array}$ & $\begin{array}{l}0,0 \\
\pm 0,0\end{array}$ & $\begin{array}{l}0,02 \\
\pm 0,06\end{array}$ & $\begin{array}{l}0,31 \\
\pm 0,25\end{array}$ & $\begin{array}{l}0,0 \\
\pm 0,0\end{array}$ & $\begin{array}{l}1,17 \\
\pm 2,05\end{array}$ & 75 & $\begin{array}{l}0,05 \\
\pm 0,14\end{array}$ & $\begin{array}{l}0,04 \\
\pm 0,06\end{array}$ & 9 \\
\hline Bühren & $26-32$ & $\begin{array}{l}0,06 \\
\pm 0,07\end{array}$ & $\begin{array}{l}0,07 \\
\pm 0,05\end{array}$ & $\begin{array}{l}4,7 \\
\pm 8,4\end{array}$ & $\begin{array}{l}0,0 \\
\pm 0,0\end{array}$ & $\begin{array}{l}0,0 \\
\pm 0,0\end{array}$ & $\begin{array}{l}0,01 \\
\pm 0,01\end{array}$ & $\begin{array}{l}0,01 \\
\pm 0,01\end{array}$ & $\begin{array}{l}4,65 \\
\pm 8,39\end{array}$ & $\begin{array}{l}0,01 \\
\pm 0,01\end{array}$ & $\begin{array}{l}0,33 \\
\pm 0,5\end{array}$ & $\begin{array}{l}0,02 \\
\pm 0,01\end{array}$ & $\begin{array}{l}4,2 \\
\pm 4,2\end{array}$ & $\begin{array}{l}0,0 \\
\pm 0,0\end{array}$ & $\begin{array}{l}0,01 \\
\pm 0,02\end{array}$ & $\begin{array}{l}0,21 \\
\pm 0,15\end{array}$ & $\begin{array}{l}0,0 \\
\pm 0,0\end{array}$ & $\begin{array}{l}1,51 \\
\pm 2,43\end{array}$ & $\begin{array}{l}0,83 \\
\pm 1,27\end{array}$ & $\begin{array}{l}0,0 \\
\pm 0,0\end{array}$ & $\begin{array}{l}0,15 \\
\pm 0,36\end{array}$ & 9 \\
\hline Reinshof & $10-13$ & $\begin{array}{l}0,62 \\
\pm 0,57\end{array}$ & n.b. & $\begin{array}{l}312 \\
\pm 75\end{array}$ & $\begin{array}{l}795 \\
\pm 142\end{array}$ & $\begin{array}{l}0,0 \\
\pm 0,0\end{array}$ & $\begin{array}{l}0,07 \\
0,0\end{array}$ & $\begin{array}{l}0,16 \\
\pm 0,10\end{array}$ & $\begin{array}{l}294 \\
\pm 89\end{array}$ & $\begin{array}{l}0,06 \\
\pm 0,02\end{array}$ & $\begin{array}{l}0,93 \\
\pm 0,10\end{array}$ & $\begin{array}{l}0,18 \\
\pm 0,02\end{array}$ & $\begin{array}{l}31 \\
\pm 22\end{array}$ & $\begin{array}{l}16 \\
\pm 14\end{array}$ & $\begin{array}{l}0,07 \\
\pm 0,03\end{array}$ & $\begin{array}{l}0,48 \\
\pm 0,35\end{array}$ & $\begin{array}{l}0,0 \\
\pm 0,0\end{array}$ & n.b. & $n h$ & n.b. & n.b. & 6 \\
\hline Reinshof & $13-14$ & $\begin{array}{l}0,74 \\
(0,9)\end{array}$ & n.b. & $\begin{array}{l}338 \\
(64)\end{array}$ & $\begin{array}{l}663 \\
(86)\end{array}$ & $\begin{array}{l}0,44 \\
(-0,1)\end{array}$ & $\begin{array}{l}0,07 \\
(0,0)\end{array}$ & $\begin{array}{l}0,15 \\
(0,0)\end{array}$ & $\begin{array}{l}300 \\
(36)\end{array}$ & $(0,0)$ & $(-0$ & $\begin{array}{l}0,18 \\
(0,06)\end{array}$ & $\begin{array}{l}53 \\
(40)\end{array}$ & $\begin{array}{l}24 \\
(29)\end{array}$ & $\begin{array}{l}0,1 \\
(0,04)\end{array}$ & $\begin{array}{l}0,51 \\
(0,49)\end{array}$ & $\begin{array}{l}0,24 \\
(0,3)\end{array}$ & i.b. & . & n.b. & n.b. & 2 \\
\hline Reinshof & $14-18$ & $\begin{array}{l}1,08 \\
\pm 0,82\end{array}$ & n.b. & $\begin{array}{l}434 \\
\pm 121\end{array}$ & $\begin{array}{l}968 \\
\pm 283\end{array}$ & $\begin{array}{l}0,0 \\
\pm 0,0\end{array}$ & $\begin{array}{l}0,03 \\
0,0\end{array}$ & $\begin{array}{l}0,07 \\
\pm 0,1\end{array}$ & $\begin{array}{l}243 \\
\pm 110\end{array}$ & $\begin{array}{l}0,02 \\
\pm 0,03\end{array}$ & $\begin{array}{l}0,57 \\
\pm 0,3\end{array}$ & $\begin{array}{l}1,25 \\
\pm 1,11\end{array}$ & $\begin{array}{l}295 \\
\pm 189\end{array}$ & $\begin{array}{l}226 \\
\pm 166\end{array}$ & $\begin{array}{l}0,06 \\
\pm 0,02\end{array}$ & $\begin{array}{l}0,36 \\
\pm 0,32\end{array}$ & $\begin{array}{l}0,0 \\
\pm 0,0\end{array}$ & n.b. & n.b. & n.b. & n.b. & 8 \\
\hline Reinshof & $18-20$ & $\begin{array}{l}2,16 \\
\pm 0,25\end{array}$ & n.b. & $\begin{array}{l}893 \\
\pm 146\end{array}$ & $\begin{array}{l}1677 \\
\pm 554\end{array}$ & $\begin{array}{l}0,0 \\
\pm 0,0\end{array}$ & $\begin{array}{l}0,04 \\
\pm 0,07\end{array}$ & $\begin{array}{l}0,07 \\
\pm 0,11\end{array}$ & $\begin{array}{l}351 \\
\pm 120\end{array}$ & $\begin{array}{l}0,02 \\
\pm 0,04\end{array}$ & $\begin{array}{l}0,4 \\
\pm 0,14\end{array}$ & $\begin{array}{l}2,86 \\
\pm 0,39\end{array}$ & $\begin{array}{l}801 \\
\pm 248\end{array}$ & $\begin{array}{l}730 \\
\pm 397\end{array}$ & $\begin{array}{l}0,23 \\
\pm 0,27\end{array}$ & $\begin{array}{l}0,35 \\
\pm 0,44\end{array}$ & $\begin{array}{l}0,0 \\
\pm 0,0\end{array}$ & I.b. & n.b. & n.b. & n.b. & 4 \\
\hline Schladen & $16-18$ & $\begin{array}{l}0,30 \\
\pm 0,45\end{array}$ & $\begin{array}{l}0,36 \\
\pm 0,64\end{array}$ & $\begin{array}{l}104 \\
\pm 12\end{array}$ & $\begin{array}{l}0,0 \\
\pm 0,0\end{array}$ & $\begin{array}{l}0,44 \\
\pm 0,14\end{array}$ & $\begin{array}{l}0,02 \\
\pm 0,01\end{array}$ & $\begin{array}{l}0,0 \\
\pm 0,0\end{array}$ & $\begin{array}{l}98 \\
\pm 0,61\end{array}$ & $\begin{array}{l}0,02 \\
\pm 0,0\end{array}$ & $\begin{array}{l}0,94 \\
\pm-0,11\end{array}$ & $\begin{array}{l}0,12 \\
\pm 0,24\end{array}$ & $\begin{array}{l}10 \\
\pm 20\end{array}$ & $\begin{array}{l}7,1 \\
\pm 14,2\end{array}$ & $\begin{array}{l}0,02 \\
\pm 0,04\end{array}$ & $\begin{array}{l}0,45 \\
\pm 0,89\end{array}$ & $\begin{array}{l}0,32 \\
\pm 0,63\end{array}$ & $\begin{array}{l}0 \\
\pm 0\end{array}$ & $\begin{array}{l}0 \\
\pm 0\end{array}$ & $\begin{array}{l}0,01 \\
\pm 0,02\end{array}$ & $\begin{array}{l}0,0 \\
\pm 0,0\end{array}$ & 9 \\
\hline Schladen & $18-24$ & $\begin{array}{l}0,9 \\
\pm 0,9\end{array}$ & $\begin{array}{l}0,82 \\
\pm 0,46\end{array}$ & $\begin{array}{l}291 \\
\pm 249\end{array}$ & $\begin{array}{l}492 \\
\pm 412\end{array}$ & $\begin{array}{l}0,41 \\
\pm 0,24\end{array}$ & $\begin{array}{l}0,02 \\
\pm 0,01\end{array}$ & $\begin{array}{l}0,03 \\
\pm 0,01\end{array}$ & $\begin{array}{l}221 \\
\pm 143\end{array}$ & $\begin{array}{l}0,01 \\
\pm 0,01\end{array}$ & $\begin{array}{l}0,84 \\
\pm 0,14\end{array}$ & $\begin{array}{l}0,24 \\
\pm 0,45\end{array}$ & $\begin{array}{l}104 \\
\pm 190\end{array}$ & $\begin{array}{l}54 \\
\pm 122\end{array}$ & $\begin{array}{l}0,17 \\
\pm 0,19\end{array}$ & $\begin{array}{l}0,71 \\
\pm 0,57\end{array}$ & $\begin{array}{l}0,43 \\
\pm 0,34\end{array}$ & $\begin{array}{l}91,9 \\
\pm 209\end{array}$ & $\begin{array}{l}258 \\
\pm 585\end{array}$ & $\begin{array}{l}0,06 \\
\pm 0,06\end{array}$ & $\begin{array}{l}0,33 \\
\pm 0,36\end{array}$ & 2 \\
\hline Schladen & $24-34$ & $\begin{array}{l}2,20 \\
\pm 1,66\end{array}$ & $\begin{array}{l}1,36 \\
\pm 0,83\end{array}$ & $\begin{array}{l}428 \\
\pm 327\end{array}$ & $\begin{array}{l}721 \\
\pm 549\end{array}$ & $\begin{array}{l}0,3 \\
\pm 0,2\end{array}$ & $\begin{array}{l}0,02 \\
\pm 0,01\end{array}$ & $\begin{array}{l}0,03 \\
\pm 0,01\end{array}$ & $\begin{array}{l}122 \\
\pm 122\end{array}$ & $\begin{array}{l}0,01 \\
\pm 0,01\end{array}$ & $\begin{array}{l}0,3 \\
\pm 0,25\end{array}$ & $\begin{array}{l}0,91 \\
\pm 1,02\end{array}$ & $\begin{array}{l}456 \\
\pm 388\end{array}$ & $\begin{array}{l}440 \\
\pm 401\end{array}$ & $\begin{array}{l}0,72 \\
\pm 0,41\end{array}$ & $\begin{array}{l}0,8 \\
\pm 0,34\end{array}$ & $\begin{array}{l}0,71 \\
\pm 0,28\end{array}$ & $\begin{array}{l}416 \\
\pm 202\end{array}$ & $\begin{array}{l}1736 \\
\pm 732\end{array}$ & $\begin{array}{l}0,31 \\
\pm 0,16\end{array}$ & $\begin{array}{l}0,82 \\
\pm 0,38\end{array}$ & 11 \\
\hline
\end{tabular}


Anhang 4 (Fortsetzung): Zusammenfassung der Ergebnisse zur Reduktion von Nitrat und Produktion von Sulfat aus den anaeroben und aeroben Inkubationsversuchen: Schichtmittelwerte der Umsatzraten zu Versuchsbeginn $\left(\mathrm{R}_{\mathrm{NO}}, \mathrm{DR}\left({ }^{15} \mathrm{~N}_{2}+\mathrm{N}_{2} \mathrm{O}\right)\right.$, $\left.\mathrm{R}_{\mathrm{SO}}\right)$, kumulierte Umsetzungen $\left(\mathrm{M}_{\mathrm{NO}}, \mathrm{M}_{\mathrm{SO} 4}\right)$, Anteil der heterotrophen Denitrifikation am Gesamtumsatz $\left(\mathrm{M}_{\mathrm{NO} 3} \mathrm{H:} \mathbf{M}_{\mathrm{NO} \text { (ges) }}\right.$, Nachhaltigkeits- und VerfügbarkeitsIndizies (NI, VI)

\begin{tabular}{|c|c|c|c|c|c|c|c|c|c|c|c|c|c|c|c|c|c|c|c|c|c|c|}
\hline tandort & $\begin{array}{l}\text { Schicht } \\
\text {-folge } \\
\text { [dm] }\end{array}$ & $\mathbf{R}_{\mathrm{NO} 3}$ & $\begin{array}{l}\text { DR } \\
\left({ }^{15} \mathrm{~N}_{2}+\mathrm{N}_{2} \mathrm{O}\right)\end{array}$ & $\begin{array}{l}\mathbf{M}_{\mathrm{NO} 3} \\
\mathbf{5 0 0 d}\end{array}$ & $\begin{array}{l}\mathbf{M}_{\mathrm{NO} 3} \\
(\infty)\end{array}$ & $\begin{array}{l}\mathrm{NI}_{\mathrm{NO3}} \\
500 \mathrm{~d}\end{array}$ & $\begin{array}{l}\mathrm{VI}_{\mathrm{NO3}} \\
\mathbf{5 0 0 d}\end{array}$ & $\begin{array}{l}\mathrm{VI}_{\mathrm{NO} 3} \\
(\infty)\end{array}$ & $\begin{array}{l}\mathrm{M}_{\mathrm{NO3}} \\
500 \mathrm{~d} \\
\mathrm{H}\end{array}$ & $\begin{array}{l}\mathrm{VI}_{\mathrm{NO3}} \\
500 \mathrm{~d} \\
\mathrm{H}\end{array}$ & $\begin{array}{l}\mathrm{M}_{\mathrm{NO} 3} \mathrm{H}: \\
\mathrm{M}_{\mathrm{NO} 3 \mathrm{ges}} \\
\mathbf{5 0 0 d}\end{array}$ & $\mathbf{R}_{\mathrm{SO} 4}$ & $\begin{array}{l}\text { Mso4 } \\
\text { 500d }\end{array}$ & $\begin{array}{l}\mathrm{M}_{\mathrm{SO} 4} \\
(\infty)\end{array}$ & $\begin{array}{l}\mathrm{Nl}_{\text {so4 }} \\
\text { 500d }\end{array}$ & $\begin{array}{l}\mathrm{VI}_{\mathrm{sO} 4} \\
\mathbf{5 0 0 0 d}\end{array}$ & $\begin{array}{l}V_{\text {SO4 }} \\
(\infty)\end{array}$ & $\begin{array}{l}\text { Mso4 } \\
500 d \\
\text { A }\end{array}$ & $\begin{array}{l}M_{\text {so4 }} \\
(\infty) \\
\text { A }\end{array}$ & $\begin{array}{l}\mathrm{NI}_{\text {SO4 }} \\
\text { 500d } \\
\text { A }\end{array}$ & $\begin{array}{l}\text { VI SO4 } \\
\text { 500d } \\
\text { A }\end{array}$ & $\mathbf{N}$ \\
\hline Fuhrberg & $\begin{array}{l}10-16 \\
36-40\end{array}$ & $\begin{array}{l}0,06 \\
\pm 0,05\end{array}$ & $\begin{array}{l}0,12 \\
\pm 0,06\end{array}$ & $\begin{array}{l}4,5 \\
\pm 5,2\end{array}$ & $\begin{array}{l}5,1 \\
\pm 5,9\end{array}$ & $\begin{array}{l}0,0 \\
\pm 0,0\end{array}$ & $\begin{array}{l}0,01 \\
\pm 0,01\end{array}$ & $\begin{array}{l}0,01 \\
\pm 0,01\end{array}$ & $\begin{array}{l}4,53 \\
\pm 5,26\end{array}$ & $\begin{array}{l}0,01 \\
\pm 0,01\end{array}$ & $\begin{array}{l}0,50 \\
\pm 0,58\end{array}$ & $\begin{array}{l}0,01 \\
\pm 0,01\end{array}$ & $\begin{array}{l}0,0 \\
\pm 0,0\end{array}$ & $\begin{array}{l}0,0 \\
\pm 0,0\end{array}$ & $\begin{array}{l}0,0 \\
\pm 0,0\end{array}$ & $\begin{array}{l}0,0 \\
\pm 0,0\end{array}$ & $\begin{array}{l}0,0 \\
\pm 0,0\end{array}$ & $\begin{array}{l}14,5 \\
\pm 29,0\end{array}$ & $\begin{array}{l}48 \\
\pm 96\end{array}$ & $\begin{array}{l}0,69 \\
\pm 1,38\end{array}$ & $\begin{array}{l}0,0 \\
\pm 0,0\end{array}$ & 4 \\
\hline Fuhrberg & $16-36$ & $\begin{array}{l}0,03 \\
\pm 0,05\end{array}$ & $\begin{array}{l}0,11 \\
\pm 0,08\end{array}$ & $\begin{array}{l}17 \\
\pm 13\end{array}$ & $\begin{array}{l}23 \\
\pm 18\end{array}$ & $\begin{array}{l}0,0 \\
\pm 0,0\end{array}$ & $\begin{array}{l}0,02 \\
\pm 0,02\end{array}$ & $\begin{array}{l}0,03 \\
\pm 0,03\end{array}$ & $\begin{array}{l}17 \\
\pm 13,3\end{array}$ & $\begin{array}{l}0,02 \\
\pm 0,02\end{array}$ & $\begin{array}{l}0,90 \\
\pm 0,32\end{array}$ & $\begin{array}{l}0,01 \\
\pm 0,0\end{array}$ & $\begin{array}{l}0,0 \\
\pm 0,0\end{array}$ & $\begin{array}{l}0,0 \\
\pm 0,0\end{array}$ & $\begin{array}{l}0,0 \\
\pm 0,0\end{array}$ & $\begin{array}{l}0,0 \\
\pm 0,0\end{array}$ & $\begin{array}{l}0,0 \\
\pm 0,0\end{array}$ & $\begin{array}{l}9,41 \\
\pm 17,0\end{array}$ & $\begin{array}{l}27 \\
\pm 31\end{array}$ & $\begin{array}{l}0,24 \\
\pm 0,5\end{array}$ & $\begin{array}{l}0,0 \\
\pm 0,0\end{array}$ & 10 \\
\hline Dinklage & $12-16$ & $\begin{array}{l}0,06 \\
\pm 0,05\end{array}$ & $\begin{array}{l}0,04 \\
\pm 0,03\end{array}$ & $\begin{array}{l}27 \\
\pm 31\end{array}$ & $\begin{array}{l}29 \\
\pm 32\end{array}$ & $\begin{array}{l}0,0 \\
\pm 0,0\end{array}$ & $\begin{array}{l}0,0 \\
\pm 0,0\end{array}$ & $\begin{array}{l}0,03 \\
\pm 0,04\end{array}$ & $\begin{array}{l}27 \\
\pm 31\end{array}$ & $\begin{array}{l}0,03 \\
\pm 0,04\end{array}$ & $\begin{array}{l}0,67 \\
\pm 0,52\end{array}$ & $\begin{array}{l}0,03 \\
\pm 0,02\end{array}$ & $\begin{array}{l}0,0 \\
\pm 0,0\end{array}$ & $\begin{array}{l}0,0 \\
\pm 0,0\end{array}$ & $\begin{array}{l}0,0 \\
\pm 0,0\end{array}$ & $\begin{array}{l}0,0 \\
\pm 0,0\end{array}$ & $\begin{array}{l}0,0 \\
\pm 0,0\end{array}$ & $\begin{array}{l}12 \\
\pm 5,46\end{array}$ & $\begin{array}{l}26 \\
\pm 16\end{array}$ & $\begin{array}{l}0,37 \\
\pm 0,1\end{array}$ & $\begin{array}{l}0,17 \\
\pm 0,41\end{array}$ & 6 \\
\hline Dinklage & $16-24$ & $\begin{array}{l}0,16 \\
\pm 0,13\end{array}$ & $\begin{array}{l}0,14 \\
\pm 0,09\end{array}$ & $\begin{array}{l}47 \\
\pm 56\end{array}$ & $\begin{array}{l}50 \\
\pm 57\end{array}$ & $\begin{array}{l}0,0 \\
\pm 0,0\end{array}$ & $\begin{array}{l}0,05 \\
\pm 0,08\end{array}$ & $\begin{array}{l}0,05 \\
\pm 0,08\end{array}$ & $\begin{array}{l}47 \\
\pm 56\end{array}$ & $\begin{array}{l}0,05 \\
\pm 0,08\end{array}$ & $\begin{array}{l}0,93 \\
\pm 0,26\end{array}$ & $\begin{array}{l}0,02 \\
\pm 0,01\end{array}$ & $\begin{array}{l}0,0 \\
\pm 0,0\end{array}$ & $\begin{array}{l}0,0 \\
\pm 0,0\end{array}$ & $\begin{array}{l}0,0 \\
\pm 0,0\end{array}$ & $\begin{array}{l}0,0 \\
\pm 0,0\end{array}$ & $\begin{array}{l}0,0 \\
\pm 0,0\end{array}$ & $\begin{array}{l}18 \\
\pm 6,83\end{array}$ & $\begin{array}{l}43 \\
\pm 18\end{array}$ & $\begin{array}{l}0,37 \\
\pm 0,11\end{array}$ & $\begin{array}{l}0,27 \\
\pm 0,57\end{array}$ & 15 \\
\hline Dinklage & $24-40$ & $\begin{array}{l}0,21 \\
\pm 0,16\end{array}$ & $\begin{array}{l}0,35 \\
\pm 0,44\end{array}$ & $\begin{array}{l}57 \\
\pm 18\end{array}$ & $\begin{array}{l}72 \\
\pm 22\end{array}$ & $\begin{array}{l}0,16 \\
\pm 0,12\end{array}$ & $\begin{array}{l}0,04 \\
\pm 0,01\end{array}$ & $\begin{array}{l}0,05 \\
\pm 0,02\end{array}$ & $\begin{array}{l}48 \\
\pm 16\end{array}$ & $\begin{array}{l}0,03 \\
\pm 0,02\end{array}$ & $\begin{array}{l}0,84 \\
\pm 0,19\end{array}$ & $\begin{array}{l}0,09 \\
\pm 0,11\end{array}$ & $\begin{array}{l}10 \\
\pm 17\end{array}$ & $\begin{array}{l}6,39 \\
\pm 7,2\end{array}$ & $\begin{array}{l}0,07 \\
\pm 0,18\end{array}$ & $\begin{array}{l}0,23 \\
\pm 0,38\end{array}$ & $\begin{array}{l}0,29 \\
\pm 0,4\end{array}$ & $\begin{array}{l}42 \\
\pm 15\end{array}$ & $\begin{array}{l}110 \\
\pm 44\end{array}$ & $\begin{array}{l}0,37 \\
\pm 0,08\end{array}$ & $\begin{array}{l}0,51 \\
\pm 0,68\end{array}$ & 10 \\
\hline $\begin{array}{l}\text { Mark- } \\
\text { hausen }\end{array}$ & $15-23$ & $\begin{array}{l}0,16 \\
\pm 0,17\end{array}$ & $\begin{array}{l}0,12 \\
\pm 0,1\end{array}$ & $\begin{array}{l}48 \\
\pm 50\end{array}$ & $\begin{array}{l}70 \\
\pm 68\end{array}$ & $\begin{array}{l}0,0 \\
\pm 0,0\end{array}$ & $\begin{array}{l}0,01 \\
\pm 0,01\end{array}$ & $\begin{array}{l}0,01 \\
\pm 0,01\end{array}$ & $\begin{array}{l}39 \\
\pm 38\end{array}$ & $\begin{array}{l}0,01 \\
\pm 0,01\end{array}$ & $\begin{array}{l}0,84 \\
\pm 0,3\end{array}$ & $\begin{array}{l}0,06 \\
\pm 0,07\end{array}$ & $\begin{array}{l}11 \\
\pm 26\end{array}$ & $\begin{array}{l}5,04 \\
\pm 8,4\end{array}$ & $\begin{array}{l}0,3 \\
\pm 0,34\end{array}$ & $\begin{array}{l}0,08 \\
\pm 0,17\end{array}$ & $\begin{array}{l}0,0 \\
\pm 0,0\end{array}$ & $\begin{array}{l}22 \\
\pm 32\end{array}$ & $\begin{array}{l}143 \\
\pm 214\end{array}$ & $\begin{array}{l}0,47 \\
\pm 0,45\end{array}$ & $\begin{array}{l}0,22 \\
\pm 0,21\end{array}$ & 12 \\
\hline $\begin{array}{l}\text { Mark- } \\
\text { hausen }\end{array}$ & 23-31 & $\begin{array}{l}0,13 \\
\pm 0,11\end{array}$ & $\begin{array}{l}0,15 \\
\pm 0,13\end{array}$ & $\begin{array}{l}49,9 \\
\pm 28\end{array}$ & $\begin{array}{l}75 \\
\pm 44\end{array}$ & $\begin{array}{l}0,0 \\
\pm 0,0\end{array}$ & $\begin{array}{l}0,05 \\
\pm 0,05\end{array}$ & $\begin{array}{l}0,08 \\
\pm 0,08\end{array}$ & $\begin{array}{l}37,3 \\
\pm 25\end{array}$ & $\begin{array}{l}0,04 \\
\pm 0,05\end{array}$ & $\begin{array}{l}0,71 \\
\pm 0,35\end{array}$ & $\begin{array}{l}0,08 \\
\pm 0,09\end{array}$ & $\begin{array}{l}19 \\
\pm 24\end{array}$ & $\begin{array}{l}7,87 \\
\pm 9,6\end{array}$ & $\begin{array}{l}0,23 \\
\pm 0,2\end{array}$ & $\begin{array}{l}0,25 \\
\pm 0,28\end{array}$ & $\begin{array}{l}0,0 \\
\pm 0,0\end{array}$ & $\begin{array}{l}41 \\
\pm 42,7\end{array}$ & $\begin{array}{l}230 \\
\pm 246\end{array}$ & $\begin{array}{l}0,54 \\
\pm 0,53\end{array}$ & $\begin{array}{l}0,65 \\
\pm 0,37\end{array}$ & 11 \\
\hline Oldendorf & $10-12$ & $\begin{array}{l}0,15 \\
\pm 0,05\end{array}$ & $\begin{array}{l}0,08 \\
\pm 0,16\end{array}$ & $\begin{array}{l}60 \\
\pm 24\end{array}$ & $\begin{array}{l}64 \\
\pm 32\end{array}$ & $\begin{array}{l}0,54 \\
\pm 0,44\end{array}$ & $\begin{array}{l}0,05 \\
\pm 0,03\end{array}$ & $\begin{array}{l}0,05 \\
\pm 0,03\end{array}$ & $\begin{array}{l}25 \\
\pm 12\end{array}$ & $\begin{array}{l}0,02 \\
\pm 0,01\end{array}$ & $\begin{array}{l}0,5 \\
\pm 0,37\end{array}$ & $\begin{array}{l}0,2 \\
\pm 0,25\end{array}$ & $\begin{array}{l}68 \\
\pm 58\end{array}$ & $\begin{array}{l}13 \\
\pm 18\end{array}$ & $\begin{array}{l}0,26 \\
\pm 0,39\end{array}$ & $\begin{array}{l}0,77 \\
\pm 0,53\end{array}$ & $\begin{array}{l}0,13 \\
\pm 0,13\end{array}$ & $\begin{array}{l}80 \\
\pm 60\end{array}$ & $\begin{array}{l}151,8 \\
\pm 125\end{array}$ & $\begin{array}{l}0,44 \\
\pm 0,22\end{array}$ & $\begin{array}{l}0,63 \\
\pm 0,45\end{array}$ & 7 \\
\hline Oldendorf & $12-14$ & $\begin{array}{l}0,16 \\
\pm 0,15\end{array}$ & $\begin{array}{l}0,04 \\
\pm 0,06\end{array}$ & $\begin{array}{l}75 \\
\pm 48\end{array}$ & $\begin{array}{l}92 \\
\pm 62\end{array}$ & $\begin{array}{l}0,0 \\
\pm 0,0\end{array}$ & $\begin{array}{l}0,04 \\
\pm 0,02\end{array}$ & $\begin{array}{l}0,05 \\
\pm 0,03\end{array}$ & $\begin{array}{l}28 \\
\pm 33\end{array}$ & $\begin{array}{l}0,01 \\
\pm 0,01\end{array}$ & $\begin{array}{l}0,41 \\
\pm 0,45\end{array}$ & $\begin{array}{l}0,28 \\
\pm 0,27\end{array}$ & $\begin{array}{l}92 \\
\pm 72\end{array}$ & $\begin{array}{l}21 \\
\pm 21\end{array}$ & $\begin{array}{l}0,14 \\
\pm 0,18\end{array}$ & $\begin{array}{l}0,66 \\
\pm 0,47\end{array}$ & $\begin{array}{l}0,0 \\
\pm 0,0\end{array}$ & $\begin{array}{l}106 \\
\pm 71\end{array}$ & $\begin{array}{l}192 \\
\pm 138\end{array}$ & $\begin{array}{l}0,46 \\
\pm 0,08\end{array}$ & $\begin{array}{l}0,79 \\
\pm 0,4\end{array}$ & 7 \\
\hline Oldendorf & $14-16$ & $\begin{array}{l}0,2 \\
\pm 0,16\end{array}$ & $\begin{array}{l}0,06 \\
\pm 0,11\end{array}$ & $\begin{array}{l}78 \\
\pm 48\end{array}$ & $\begin{array}{l}89 \\
\pm 59\end{array}$ & $\begin{array}{l}0,0 \\
\pm 0,0\end{array}$ & $\begin{array}{l}0,06 \\
\pm 0,05\end{array}$ & $\begin{array}{l}0,07 \\
\pm 0,06\end{array}$ & $\begin{array}{l}25 \\
\pm 25\end{array}$ & $\begin{array}{l}0,01 \\
\pm 0,01\end{array}$ & $\begin{array}{l}0,45 \\
\pm 0,47\end{array}$ & $\begin{array}{l}0,39 \\
\pm 0,34\end{array}$ & $\begin{array}{r}111 \\
\pm 95\end{array}$ & $\begin{array}{l}30 \\
\pm 25\end{array}$ & $\begin{array}{l}0,07 \\
\pm 0,07\end{array}$ & $\begin{array}{l}0,67 \\
\pm 0,6\end{array}$ & $\begin{array}{l}0,0 \\
\pm 0,0\end{array}$ & $\begin{array}{r}137 \\
\pm 99\end{array}$ & $\begin{array}{l}242 \\
\pm 163\end{array}$ & $\begin{array}{l}0,55 \\
\pm 0,24\end{array}$ & $\begin{array}{l}0,89 \\
\pm 0,48\end{array}$ & 6 \\
\hline Vechtel & $8-12$ & $\begin{array}{l}0,14 \\
\pm 0,13\end{array}$ & $\begin{array}{l}0,01 \\
\pm 0,0\end{array}$ & $\begin{array}{l}37 \\
\pm 33\end{array}$ & $\begin{array}{l}38 \\
\pm 32\end{array}$ & $\begin{array}{l}0,0 \\
\pm 0,0\end{array}$ & $\begin{array}{l}0,04 \\
\pm 0,03\end{array}$ & $\begin{array}{l}0,04 \\
\pm 0,03\end{array}$ & $\begin{array}{l}37 \\
\pm 33\end{array}$ & $\begin{array}{l}0,04 \\
\pm 0,03\end{array}$ & $\begin{array}{l}0,89 \\
\pm 0,33\end{array}$ & $\begin{array}{l}0,02 \\
\pm 0,01\end{array}$ & $\begin{array}{l}0,0 \\
\pm 0,0\end{array}$ & $\begin{array}{l}0,0 \\
\pm 0,0\end{array}$ & $\begin{array}{l}0,0 \\
\pm 0,0\end{array}$ & $\begin{array}{l}0,0 \\
\pm 0,0\end{array}$ & $\begin{array}{l}0,0 \\
\pm 0,0\end{array}$ & $\begin{array}{l}4,03 \\
\pm 2,59\end{array}$ & $\begin{array}{l}12,1 \\
\pm 10,2\end{array}$ & $\begin{array}{l}0,07 \\
\pm 0,11\end{array}$ & $\begin{array}{l}0,74 \\
\pm 0,44\end{array}$ & 9 \\
\hline Vechtel & $12-14$ & $\begin{array}{l}0,1 \\
\pm 0,06\end{array}$ & $\begin{array}{l}0,0 \\
\pm 0,0\end{array}$ & $\begin{array}{l}39 \\
\pm 23\end{array}$ & $\begin{array}{l}40 \\
\pm 23\end{array}$ & $\begin{array}{l}0,0 \\
\pm 0,0\end{array}$ & $\begin{array}{l}0,05 \\
\pm 0,04\end{array}$ & $\begin{array}{l}0,05 \\
\pm 0,04\end{array}$ & $\begin{array}{l}39 \\
\pm 23\end{array}$ & $\begin{array}{l}0,05 \\
\pm 0,04\end{array}$ & $\begin{array}{l}1,0 \\
0,0\end{array}$ & $\begin{array}{l}0,01 \\
\pm 0,0\end{array}$ & $\begin{array}{l}0,0 \\
\pm 0,0\end{array}$ & $\begin{array}{l}0,0 \\
\pm 0,0\end{array}$ & $\begin{array}{l}0,0 \\
\pm 0,0\end{array}$ & $\begin{array}{l}0,0 \\
\pm 0,0\end{array}$ & $\begin{array}{l}0,0 \\
\pm 0,0\end{array}$ & $\begin{array}{l}3,34 \\
\pm 3,07\end{array}$ & $\begin{array}{l}6,0 \\
\pm 4,68\end{array}$ & $\begin{array}{l}0,05 \\
\pm 0,05\end{array}$ & $\begin{array}{l}0,6 \\
\pm 0,55\end{array}$ & 5 \\
\hline
\end{tabular}


Anhang 4 (Fortsetzung): Zusammenfassung der Ergebnisse zur Reduktion von Nitrat und Produktion von Sulfat aus den anaeroben und aeroben Inkubationsversuchen: Schichtmittelwerte der Umsatzraten zu Versuchsbeginn $\left(\mathbf{R}_{\mathrm{NO}}, \mathrm{DR}^{15}\left(\mathbf{N}_{2}+\mathbf{N}_{2} \mathrm{O}\right)\right.$, $\left.\mathbf{R}_{\mathrm{SO}}\right)$, kumulierte Umsetzungen $\left(M_{\mathrm{NO} 3}, M_{\mathrm{SO} 4}\right)$, Anteil der heterotrophen Denitrifikation am Gesamtumsatz $\left(\mathrm{M}_{\mathrm{NO}} \mathrm{H}: \mathrm{M}_{\mathrm{NO} \text { (ges) }}\right)$, Nachhaltigkeits- und VerfügbarkeitsIndizies (NI, VI)

\begin{tabular}{|c|c|c|c|c|c|c|c|c|c|c|c|c|c|c|c|c|c|c|c|c|c|c|}
\hline Standort & $\begin{array}{l}\text { Schicht } \\
\text {-folge } \\
\text { [dm] }\end{array}$ & $\mathbf{R}_{\mathrm{NO} 3}$ & $\begin{array}{l}\text { DR } \\
\left({ }^{15} \mathrm{~N}_{2}+\mathrm{N}_{2} \mathrm{O}\right)\end{array}$ & $\begin{array}{l}M_{\mathrm{NO} 3} \\
500 d\end{array}$ & $\begin{array}{l}M_{\mathrm{NO} 3} \\
(\infty)\end{array}$ & $\begin{array}{l}\mathrm{NI}_{\mathrm{NO3}} \\
500 \mathrm{~d}\end{array}$ & $\begin{array}{l}\mathrm{VI}_{\mathrm{NO} 3} \\
500 \mathrm{~d}\end{array}$ & $\begin{array}{l}\mathrm{VI}_{\mathrm{NO3}} \\
(\infty)\end{array}$ & $\begin{array}{l}M_{\mathrm{NO} 3} \\
500 d \\
\mathrm{H}\end{array}$ & $\begin{array}{l}\mathrm{VI}_{\mathrm{NO} 3} \\
500 \mathrm{~d} \\
\mathrm{H}\end{array}$ & $\begin{array}{l}\mathrm{M}_{\mathrm{NO} 3} \mathrm{H}: \\
\mathrm{M}_{\mathrm{NO} 3 \mathrm{ges}} \\
500 \mathrm{~d}\end{array}$ & $\mathbf{R}_{\mathrm{SO} 4}$ & $\begin{array}{l}\mathrm{M}_{\mathrm{SO} 4} \\
500 \mathrm{~d}\end{array}$ & $\begin{array}{l}\mathrm{M}_{\mathrm{SO} 4} \\
(\infty)\end{array}$ & $\begin{array}{l}\mathrm{NI}_{\mathrm{SO} 4} \\
500 \mathrm{~d}\end{array}$ & $\begin{array}{l}\mathrm{VI}_{\mathrm{SO} 4} \\
500 \mathrm{~d}\end{array}$ & $\begin{array}{l}\mathrm{VI}_{\mathrm{SO} 4} \\
(\infty)\end{array}$ & $\begin{array}{l}M_{\mathrm{SO} 4} \\
500 \mathrm{~d} \\
\mathrm{~A}\end{array}$ & $\begin{array}{l}M_{\mathrm{SO} 4} \\
(\infty) \\
\text { A }\end{array}$ & $\begin{array}{l}\mathrm{NI}_{\mathrm{SO} 4} \\
500 \mathrm{~d} \\
\mathrm{~A}\end{array}$ & $\begin{array}{l}\mathrm{Vl}_{\mathrm{SO} 4} \\
500 \mathrm{~d} \\
\mathrm{~A}\end{array}$ & $\mathbf{N}$ \\
\hline $\begin{array}{l}\text { Neuhäu- } \\
\text { ser Felde }\end{array}$ & $10-24$ & $\begin{array}{l}0,07 \\
\pm 0,12\end{array}$ & $\begin{array}{l}0,04 \\
\pm 0,05\end{array}$ & $\begin{array}{l}41 \\
\pm 37\end{array}$ & $\begin{array}{l}46 \\
\pm 37\end{array}$ & $\begin{array}{l}0,42 \\
\pm 0,63\end{array}$ & $\begin{array}{l}0,02 \\
\pm 0,02\end{array}$ & $\begin{array}{l}0,02 \\
\pm 0,02\end{array}$ & $\begin{array}{l}40 \\
\pm 37\end{array}$ & $\begin{array}{l}0,02 \\
\pm 0,02\end{array}$ & $\begin{array}{l}0,69 \\
\pm 0,45\end{array}$ & $\begin{array}{l}0,09 \\
\pm 0,07\end{array}$ & $\begin{array}{l}0,0 \\
\pm 0,0\end{array}$ & $\begin{array}{l}2,31 \\
\pm 5,0\end{array}$ & $\begin{array}{l}0,28 \\
\pm 0,15\end{array}$ & $\begin{array}{l}0,0 \\
\pm 0,0\end{array}$ & $\begin{array}{l}0,08 \\
\pm 0,17\end{array}$ & $\begin{array}{l}0,39 \\
\pm 0,64\end{array}$ & $\begin{array}{l}0,33 \\
\pm 0,68\end{array}$ & $\begin{array}{l}0,10 \\
\pm 0,23\end{array}$ & $\begin{array}{l}0,07 \\
\pm 0,16\end{array}$ & 21 \\
\hline $\begin{array}{l}\text { Neuhäu- } \\
\text { ser Felde }\end{array}$ & $24-36$ & $\begin{array}{l}2,28 \\
\pm 1,32\end{array}$ & $\begin{array}{l}2,41 \\
\pm 2,2\end{array}$ & $\begin{array}{l}271 \\
\pm 159\end{array}$ & $\begin{array}{l}280 \\
\pm 167\end{array}$ & $\begin{array}{l}0,11 \\
\pm 0,2\end{array}$ & $\begin{array}{l}0,05 \\
\pm 0,02\end{array}$ & $\begin{array}{l}0,05 \\
\pm 0,02\end{array}$ & $\begin{array}{l}97 \\
\pm 57\end{array}$ & $\begin{array}{l}0,02 \\
\pm 0,01\end{array}$ & $\begin{array}{l}0,39 \\
\pm 0,14\end{array}$ & $\begin{array}{l}0,98 \\
\pm 0,68\end{array}$ & $\begin{array}{l}279 \\
\pm 201\end{array}$ & $\begin{array}{l}142 \\
\pm 121\end{array}$ & $\begin{array}{l}0,14 \\
\pm 0,08\end{array}$ & $\begin{array}{l}0,36 \\
\pm 0,31\end{array}$ & $\begin{array}{l}0,16 \\
\pm 0,12\end{array}$ & $\begin{array}{l}454 \\
\pm 213\end{array}$ & $\begin{array}{l}2024 \\
\pm 942\end{array}$ & $\begin{array}{l}0,33 \\
\pm 0,1\end{array}$ & $\begin{array}{l}0,36 \\
\pm 0,17\end{array}$ & 14 \\
\hline $\begin{array}{l}\text { Neuhäu- } \\
\text { ser Felde }\end{array}$ & $36-40$ & $\begin{array}{l}1,63 \\
\pm 0,73\end{array}$ & $\begin{array}{l}2,36 \\
\pm 1,37\end{array}$ & $\begin{array}{l}197 \\
\pm 51\end{array}$ & $\begin{array}{l}198 \\
\pm 51\end{array}$ & $\begin{array}{l}0,08 \\
\pm 0,04\end{array}$ & $\begin{array}{l}0,04 \\
\pm 0,0\end{array}$ & $\begin{array}{l}0,04 \\
\pm 0,0\end{array}$ & $\begin{array}{l}117 \\
\pm-14\end{array}$ & $\begin{array}{l}0,03 \\
\pm 0,01\end{array}$ & $\begin{array}{l}0,61 \\
\pm-0,23\end{array}$ & $\begin{array}{l}0,48 \\
\pm 0,2\end{array}$ & $\begin{array}{l}130 \\
\pm 105\end{array}$ & $\begin{array}{l}59 \\
\pm 44\end{array}$ & $\begin{array}{l}0,22 \\
\pm 0,06\end{array}$ & $\begin{array}{l}0,1 \\
\pm 0,09\end{array}$ & $\begin{array}{l}0,05 \\
\pm 0,04\end{array}$ & $\begin{array}{l}627 \pm 9 \\
7\end{array}$ & $\begin{array}{l}3344 \\
\pm 385\end{array}$ & $\begin{array}{l}0,41 \\
\pm 0,05\end{array}$ & $\begin{array}{l}0,5 \\
\pm 0,1\end{array}$ & 2 \\
\hline Freistatt & $14-30$ & $\begin{array}{l}0,09 \\
\pm 0,08\end{array}$ & $\begin{array}{l}0,20 \\
\pm 0,22\end{array}$ & $\begin{array}{l}40 \\
\pm 29\end{array}$ & $\begin{array}{l}50 \\
\pm 40\end{array}$ & $\begin{array}{l}0,34 \\
\pm 0,4\end{array}$ & $\begin{array}{l}0,02 \\
\pm 0,02\end{array}$ & $\begin{array}{l}0,03 \\
\pm 0,02\end{array}$ & $\begin{array}{l}40 \\
\pm 29\end{array}$ & $\begin{array}{l}0,02 \\
\pm 0,02\end{array}$ & $\begin{array}{l}1,0 \\
\pm 0,0\end{array}$ & $\begin{array}{l}0,01 \\
\pm 0,01\end{array}$ & $\begin{array}{l}0,0 \\
\pm 0,0\end{array}$ & $\begin{array}{l}0,0 \\
\pm 0,0\end{array}$ & $\begin{array}{l}0,0 \\
\pm 0,0\end{array}$ & $\begin{array}{l}0,0 \\
\pm 0,0\end{array}$ & $\begin{array}{l}0,0 \\
\pm 0,0\end{array}$ & $\begin{array}{l}2,41 \\
\pm 0,97\end{array}$ & $\begin{array}{l}0,10 \\
\pm 0,18\end{array}$ & $\begin{array}{l}0,52 \\
\pm 0,55\end{array}$ & $\begin{array}{l}0,19 \\
\pm 0,18\end{array}$ & 7 \\
\hline Freistatt & $16-30$ & $\begin{array}{l}0,06 \\
\pm 0,04\end{array}$ & $\begin{array}{l}0,14 \\
\pm 0,08\end{array}$ & $\begin{array}{l}7,3 \\
\pm 7,9\end{array}$ & $\begin{array}{l}9,54 \\
\pm 7,0\end{array}$ & $\begin{array}{l}0,45 \\
\pm 0,62\end{array}$ & $\begin{array}{l}0,01 \\
\pm 0,01\end{array}$ & $\begin{array}{l}0,01 \\
\pm 0,01\end{array}$ & $\begin{array}{l}7,28 \\
\pm 6,88\end{array}$ & $\begin{array}{l}0,01 \\
\pm 0,01\end{array}$ & $\begin{array}{l}1,0 \\
\pm 0,0\end{array}$ & $\begin{array}{l}0,01 \\
\pm 0,01\end{array}$ & $\begin{array}{l}0,0 \\
\pm 0,0\end{array}$ & $\begin{array}{l}0,0 \\
\pm 0,0\end{array}$ & $\begin{array}{l}0,0 \\
\pm 0,0\end{array}$ & $\begin{array}{l}0,0 \\
\pm 0,0\end{array}$ & $\begin{array}{l}0,0 \\
\pm 0,0\end{array}$ & $\begin{array}{l}3,67 \\
\pm 1,10\end{array}$ & $\begin{array}{l}0,0 \\
\pm 0,0\end{array}$ & $\begin{array}{l}0,49 \\
\pm 0,69\end{array}$ & $\begin{array}{l}0,81 \\
\pm 0,21\end{array}$ & 7 \\
\hline Freistatt & $16-30$ & $\begin{array}{l}0,02 \\
\pm 0,04\end{array}$ & $\begin{array}{l}0,12 \\
\pm 0,13\end{array}$ & $\begin{array}{l}12 \\
\pm 11\end{array}$ & $\begin{array}{l}15 \\
\pm 13\end{array}$ & $\begin{array}{l}0,47 \\
\pm 0,62\end{array}$ & $\begin{array}{l}0,02 \\
\pm 0,01\end{array}$ & $\begin{array}{l}0,02 \\
\pm 0,02\end{array}$ & $\begin{array}{l}11,7 \\
\pm 11\end{array}$ & $\begin{array}{l}0,02 \\
\pm 0,01\end{array}$ & $\begin{array}{l}0,69 \\
\pm 0,48\end{array}$ & $\begin{array}{l}0,02 \\
\pm 0,01\end{array}$ & $\begin{array}{l}0,0 \\
\pm 0,0\end{array}$ & $\begin{array}{l}0,0 \\
\pm 0,0\end{array}$ & $\begin{array}{l}0,0 \\
\pm 0,0\end{array}$ & $\begin{array}{l}0,0 \\
\pm 0,0\end{array}$ & $\begin{array}{l}0,0 \\
\pm 0,0\end{array}$ & $\begin{array}{l}7,44 \\
\pm 1,38\end{array}$ & $\begin{array}{l}0,0 \\
\pm 0,0\end{array}$ & $\begin{array}{l}0,03 \\
\pm 0,05\end{array}$ & $\begin{array}{l}0,96 \\
\pm 0,56\end{array}$ & 7 \\
\hline
\end{tabular}

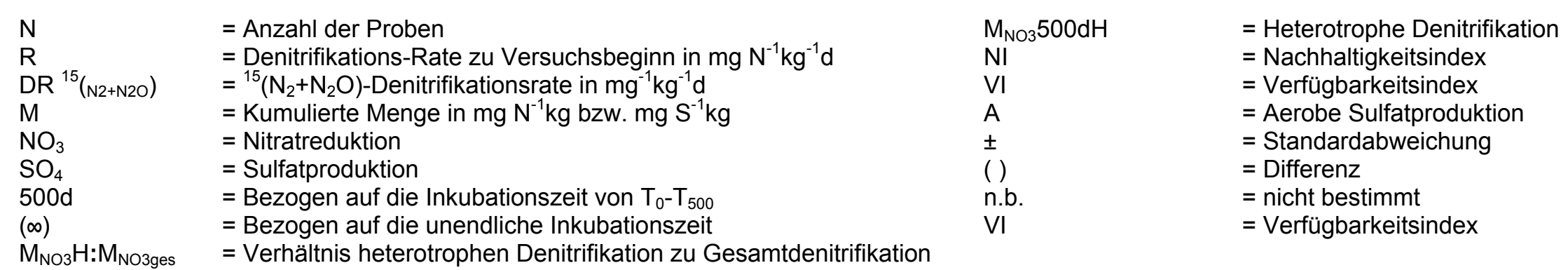


Anhang 5: Ergebnisse der linearen multiplen Regressionsanlyse zwischen unabhängigen und abhängigen Koeffizienten der Regressionsgeraden mit statistischen Kenngrößen

KOEFFIZIENTEN - - STATISTIK

\begin{tabular}{|c|c|c|c|c|c|c|c|c|c|c|c|c|c|c|c|c|}
\hline Parameter & $\begin{array}{l}\text { Ver- } \\
\text { fahren }\end{array}$ & Konstante & $\begin{array}{l}\text { Log } \\
\mathrm{C}_{\text {org }} \\
\%\end{array}$ & $C_{\text {lös }}$ & $\mathbf{C}_{\mathrm{hwl}}$ & $\begin{array}{l}\log N_{t} \\
\mathrm{mg} / \mathbf{k g}\end{array}$ & $\begin{array}{l}\text { Log } \\
\text { Sulfid }\end{array}$ & $\begin{array}{l}\text { Log } \\
F_{e_{\text {lös }}}\end{array}$ & $\begin{array}{l}\mathrm{pH} \\
\left(\mathrm{CaCl}_{2}\right)\end{array}$ & $\mathrm{CaCO}_{3}$ & Sand & $\begin{array}{r}\text { Log } \\
\text { Ton } \\
\%\end{array}$ & $\begin{array}{l}\text { Log } \\
\text { Schluff }\end{array}$ & $\mathbf{R}^{2}$ & $\mathbf{P}$ & $\mathbf{N}$ \\
\hline $\log \left[\mathrm{R}_{\mathrm{NO} \text { (Alle) }}\right]$ & $S$ & $-1,01$ & 0,84 & & & & 0,20 & & & & & & & 0,24 & $1,0 \mathrm{E}-14$ & 233 \\
\hline $\log \left[\mathrm{R}_{\mathrm{NO} \text { (Alle) }}\right]$ & $D$ & $-1,12$ & 1,31 & & & $-0,39$ & 0,20 & & 0,16 & & $-0,01$ & $-0,84$ & & 0,26 & $7,3 \mathrm{E}-14$ & 233 \\
\hline $\mathrm{M}_{\mathrm{NO} \text { (Alle) } 500 \mathrm{~d}}$ & $S$ & 120 & 243 & & & & 14,2 & & 26,5 & & & & & 0,63 & 9,9E-50 & 233 \\
\hline $\mathrm{M}_{\mathrm{NO3(Alle)}} 500 \mathrm{~d}$ & D & 148 & 230 & & & 13,9 & 14 & & 25,9 & & $-0,01$ & $-2,20$ & & 0,62 & $1,1 \mathrm{E}-46$ & 233 \\
\hline $\log \left[\mathrm{M}_{\mathrm{NO} 3(\mathrm{All})}(\infty)\right]$ & $S$ & 0,40 & & & & 1,65 & 0,27 & & 0,35 & & 0,03 & & & 0,36 & $5,9 E-22$ & 233 \\
\hline $\log \left[M_{\text {NO3(Alle) }}(\infty)\right]$ & D & $-0,15$ & 0,68 & & & 1,11 & 0,24 & & 0,37 & & 0,03 & & & 0,36 & $5,9 \mathrm{E}-21$ & 233 \\
\hline $\log \left[\mathrm{NI}_{\mathrm{NO}(\mathrm{Alle})} 500 \mathrm{~d}\right]$ & $S$ & $-0,26$ & 1,20 & & & & 0,14 & & & & & $-0,93$ & & 0,21 & $2,4 \mathrm{E}-12$ & 233 \\
\hline $\log \left[\mathrm{NI}_{\mathrm{NO} 3(\mathrm{All}) \text { ) }} 500 \mathrm{~d}\right]$ & $D$ & $-2,46$ & 1,14 & & & 0,03 & 0,15 & & 0,22 & & 0,01 & $-0,46$ & & 0,21 & $4,7 E-11$ & 233 \\
\hline $\mathrm{VI}_{\mathrm{NO} 3(\mathrm{All}) \text { ) }} 500 \mathrm{~d}$ & $S$ & $-0,02$ & $-0,01$ & & & & & & 0,01 & & & & & 0,10 & $1,5 \mathrm{E}-05$ & 233 \\
\hline $\mathrm{VI}_{\mathrm{NO} \text { (Alle) }} 500 \mathrm{~d}$ & D & 0,01 & $-0,03$ & & & 0,01 & & & 0,01 & & & $-0,02$ & & 0,10 & 3,1E-05 & 233 \\
\hline $\log \left[\mathrm{VI}_{\mathrm{NO}(\mathrm{Alle})}(\infty)\right]$ & $S$ & $-1,84$ & & & & & 0,12 & & & & & & & 0,07 & $3,2 \mathrm{E}-05$ & 233 \\
\hline $\log \left[\mathrm{VI}_{\mathrm{NO} 3(\mathrm{All})}(\infty)\right]$ & D & $-2,26$ & $-0,32$ & & & 0,43 & 0,12 & & 0,14 & & 0,01 & $-0,12$ & & 0,11 & 1,6E-05 & 233 \\
\hline $\log \left[R_{\text {SO4(Alle }}{ }^{*}\right]$ & $S$ & $-1,44$ & & & & & 0,25 & & 0,09 & & & & & 0,29 & $7,0 \mathrm{E}-09$ & 108 \\
\hline $\log \left[\mathrm{R}_{\text {SO4(Alle) }}{ }^{*}\right]$ & $D$ & $-1,17$ & $-0,24$ & & & 0,38 & 0,22 & & 0,11 & & 0,01 & & & 0,27 & $7,2 \mathrm{E}-07$ & 108 \\
\hline $\mathrm{M}_{\mathrm{SO} 4 \text { (Alle) }}{ }^{*} 500 \mathrm{~d}$ & $S$ & 22,5 & 106 & $-1,90$ & & & 68,02 & & & & & & & 0,44 & $1,0 \mathrm{E}-13$ & 108 \\
\hline $\mathrm{M}_{\text {SO4(Alle) }}{ }^{*} 500 \mathrm{~d}$ & D & 16,0 & 121 & $-1,64$ & & 1,40 & 68,29 & & 1,16 & & 0,48 & $-26,6$ & & 0,43 & $2,5 \mathrm{E}-11$ & 108 \\
\hline $\mathrm{M}_{\text {SO4(Alle) }}{ }^{*}(\infty)$ & $S$ & 56,4 & 133 & $-2,46$ & & & 44,34 & & & & & & & 0,44 & $1,1 \mathrm{E}-13$ & 108 \\
\hline $\mathrm{M}_{\text {SO4(Alle) }}{ }^{*}(\infty)$ & D & 53,7 & 123 & $-2,20$ & & 13,85 & 47,36 & & $-10,77$ & & 1,43 & 56 & & 0,44 & $7,5 \mathrm{E}-12$ & 108 \\
\hline $\mathrm{NI}_{\text {SO4(Alle) }}{ }^{*} 500 \mathrm{~d}$ & $S$ & 0,20 & 0,11 & & & & & & & $-0,01$ & & & & 0,12 & 4,4E-04 & 108 \\
\hline $\mathrm{NI}_{\mathrm{SO} 4 \text { (Alle) }}{ }^{*} 500 \mathrm{~d}$ & D & $-0,22$ & 0,02 & & & 0,10 & $-0,04$ & & 0,01 & 0,00 & 0,01 & 0,46 & & 0,21 & 5,4E-05 & 108 \\
\hline $\mathrm{VI}_{\text {SO4(Alle) }}{ }^{*} 500 \mathrm{~d}$ & $S$ & 0,36 & & & & & 0,00 & & & $-0,03$ & & & & 0,09 & 1,2E-03 & 108 \\
\hline $\mathrm{VI}_{\text {SO4(Alle) }}{ }^{*} 500 \mathrm{~d}$ & $D$ & 0,20 & 0,01 & & & $-0,10$ & 0,06 & & 0,01 & $-0,04$ & & $-0,13$ & & 0,08 & 3,7E-02 & 108 \\
\hline $\mathrm{VI}_{\mathrm{SO} 4(\mathrm{Alle})^{*}}(\infty)$ & $S$ & 0,25 & & & & & 0,00 & & $-0,05$ & $-0,02$ & & & 0,31 & 0,26 & 1,7E-07 & 108 \\
\hline $\mathrm{VI}_{\mathrm{SO} 4(\mathrm{All} e)^{*}}(\infty)$ & D & $-0,03$ & $-0,08$ & & & 0,02 & $-0,01$ & & $-0,05$ & $-0,02$ & & 0,32 & 0,34 & 0,27 & 2,8E-06 & 108 \\
\hline
\end{tabular}


Anhang 5 (Fortsetzung): Ergebnisse der linearen multiplen Regressionsanlyse zwischen unabhängigen und abhängigen Koeffizienten der Regressionsgeraden mit statistischen Kenngrößen.

KOEFFIZIENTEN

STATISTIK

\begin{tabular}{|c|c|c|c|c|c|c|c|c|c|c|c|c|c|c|c|c|}
\hline Parameter & $\begin{array}{l}\text { Ver- } \\
\text { fahren }\end{array}$ & Konstante & $\begin{array}{l}\text { Log } \\
\text { C }_{\text {org }} \\
\%\end{array}$ & $\mathbf{C}_{\text {lös }}$ & $\mathrm{C}_{\mathrm{hwl}}$ & $\begin{array}{l}\log N_{t} \\
\mathrm{mg} / \mathrm{kg}\end{array}$ & $\begin{array}{l}\text { Log } \\
\text { Sulfid }\end{array}$ & $\begin{array}{l}\text { Log } \\
F e_{\text {lös }} \\
\end{array}$ & $\begin{array}{l}\mathrm{pH} \\
\left(\mathrm{CaCl}_{2}\right)\end{array}$ & $\mathrm{CaCO}_{3}$ & Sand & $\begin{array}{l}\text { Log } \\
\text { Ton } \\
\%\end{array}$ & $\begin{array}{l}\text { Log } \\
\text { Schluff }\end{array}$ & $\mathbf{R}^{2}$ & $\mathbf{P}$ & $\mathbf{N}$ \\
\hline $\log \left[\mathrm{R}_{\mathrm{NO}(\mathrm{S})}\right]$ & $S$ & $-1,45$ & & & & & 0,19 & & & & & $-1,03$ & & 0,09 & 1,0E-03 & 123 \\
\hline $\log \left[\mathrm{R}_{\mathrm{NO}(\mathrm{S})}\right]$ & $D$ & $-1,84$ & 1,49 & & & $-0,99$ & 0,15 & & 0,49 & & $-0,03$ & $-1,05$ & & 0,15 & 2,6E-04 & 123 \\
\hline $\mathrm{M}_{\mathrm{NO} 3(\mathrm{~S})} 500 \mathrm{~d}$ & $S$ & 95 & 48 & $-0,88$ & & & 4,15 & & & 205 & & & & 0,25 & 8,1E-08 & 123 \\
\hline $\mathrm{M}_{\mathrm{NO} 3(\mathrm{~S})} 500 \mathrm{~d}$ & D & 66 & 58 & $-0,82$ & & -15 & 4,22 & & 3,39 & 196 & $-0,10$ & $-14,7$ & & 0,25 & 1,7E-06 & 123 \\
\hline $\log \left[\mathrm{M}_{\mathrm{NO}(\mathrm{S})}(\infty)\right]$ & $\mathrm{S}$ & $-1,90$ & & & & & 0,34 & & 0,60 & & & & & 0,16 & $9,9 E-06$ & 123 \\
\hline $\log \left[\mathrm{M}_{\mathrm{NO}(\mathrm{S})}(\infty)\right]$ & $D$ & $-1,88$ & 1,19 & & & $-0,69$ & 0,30 & & 0,75 & & $-0,01$ & 0,04 & & 0,17 & 1,3E-04 & 123 \\
\hline $\log \left[\mathrm{NI}_{\mathrm{NO}(\mathrm{S})} 500 \mathrm{~d}\right]$ & $S$ & $-1,51$ & & & & & 0,23 & & & & & & & 0,06 & 4,0E-03 & 123 \\
\hline $\log \left[\mathrm{NI}_{\mathrm{NO}(\mathrm{S})} 500 \mathrm{~d}\right]$ & D & $-4,68$ & 1,07 & & & $-0,42$ & 0,21 & & 0,60 & & & 0,25 & & 0,08 & $1,9 \mathrm{E}-02$ & 123 \\
\hline $\log \left[\mathrm{VI}_{\mathrm{NO} 3(\mathrm{~S})} 500 \mathrm{~d}\right]$ & $S$ & $-3,99$ & & & & & 0,12 & & 0,44 & & & & & 0,11 & 2,7E-04 & 123 \\
\hline $\log \left[\mathrm{VI}_{\mathrm{NO}(\mathrm{S})} 500 \mathrm{~d}\right]$ & D & $-3,51$ & $-0,13$ & & & $-0,43$ & 0,14 & & 0,36 & & $-0,01$ & $-0,34$ & & 0,14 & 7,2E-04 & 123 \\
\hline $\log \left[\mathrm{VI}_{\mathrm{NO}(\mathrm{S})}(\infty)\right]$ & S & $-3,63$ & & & & & 0,13 & & 0,38 & & & & & 0,12 & $1,5 \mathrm{E}-04$ & 123 \\
\hline $\log \left[\mathrm{VI}_{\mathrm{NO}(\mathrm{S})}(\infty)\right]$ & D & $-3,33$ & $-0,16$ & & & $-0,35$ & 0,15 & & 0,30 & & $-0,01$ & $-0,30$ & & 0,14 & 7,7E-04 & 123 \\
\hline $\log \left[\mathrm{R}_{\mathrm{NO}(\mathrm{L})}\right]$ & $S$ & $-1,26$ & 0,81 & & & & 0,32 & & & & & & & 0,28 & 9,9E-09 & 110 \\
\hline $\log \left[R_{\mathrm{NO} 3(\mathrm{~L})}\right]$ & $D$ & $-2,29$ & 1,14 & & & $-0,33$ & 0,31 & & 0,13 & & & $-0,08$ & & 0,26 & $1,4 \mathrm{E}-06$ & 110 \\
\hline $\mathrm{M}_{\mathrm{NO} 3(\mathrm{~L})} 500 \mathrm{~d}$ & $S$ & 245 & 270 & & & & 41,0 & & & & & & & 0,62 & $1,3 \mathrm{E}-23$ & 110 \\
\hline $\mathrm{M}_{\mathrm{NO}(\mathrm{L})} 500 \mathrm{~d}$ & D & 69,0 & 368 & & & -88 & 39,0 & & 21,0 & & $-1,01$ & $-21,1$ & & 0,63 & $3,6 \mathrm{E}-21$ & 110 \\
\hline $\log \left[\mathrm{M}_{\mathrm{NO}(\mathrm{L})}(\infty)\right]$ & S & 9,22 & & & & 2,33 & & & 0,56 & & & & $-4,69$ & 0,51 & $4,6 \mathrm{E}-17$ & 110 \\
\hline $\log \left[\mathrm{M}_{\mathrm{NO}(\mathrm{L})}(\infty)\right]$ & D & $-6,18$ & 0,73 & & & 1,61 & 0,11 & & 0,58 & & 0,05 & 2,31 & 1,21 & 0,51 & $5,0 \mathrm{E}-15$ & 110 \\
\hline $\log \left[\mathrm{NI}_{\mathrm{NO}(\mathrm{L})} 500 \mathrm{~d}\right]$ & $\mathrm{S}$ & $-3,26$ & 1,76 & & & & & & 0,34 & & & & & 0,42 & $7,4 \mathrm{E}-14$ & 110 \\
\hline $\log \left[\mathrm{NI}_{\mathrm{NO} 3(\mathrm{~L})} 500 \mathrm{~d}\right]$ & D & $-2,62$ & 1,49 & & & 0,22 & 0,06 & & 0,30 & & & $-0,14$ & & 0,40 & $4,3 \mathrm{E}-11$ & 110 \\
\hline $\log \left[\mathrm{VI}_{\mathrm{NO} 3(\mathrm{~L})} 500 \mathrm{~d}\right]$ & $S$ & $-1,07$ & & & & 0,63 & & & & & & & & 0,24 & $3,8 \mathrm{E}-08$ & 110 \\
\hline $\log \left[\mathrm{VI}_{\mathrm{NO} 3(\mathrm{~L})} 500 \mathrm{~d}\right]$ & D & $-2,25$ & $-0,23$ & & & 0,71 & 0,13 & & 0,11 & & & 0,25 & & 0,26 & $1,4 \mathrm{E}-06$ & 110 \\
\hline $\log \left[\mathrm{VI}_{\mathrm{NO}(\mathrm{L})}(\infty)\right]$ & S & $-1,92$ & & & & 0,51 & & & 0,14 & & & & & 0,20 & 2,4E-06 & 110 \\
\hline $\log \left[\mathrm{VI}_{\mathrm{NO}(\mathrm{L})}(\infty)\right]$ & D & $-4,09$ & $-0,32$ & & & 0,67 & 0,07 & & 0,23 & & 0,01 & 1,07 & & 0,24 & 4,7E-06 & 110 \\
\hline
\end{tabular}


Anhang 5 (Fortsetzung): Ergebnisse der linearen multiplen Regressionsanlyse zwischen unabhängigen und abhängigen Koeffizienten der Regressionsgeraden mit statistischen Kenngrößen.

KOEFFIZIENTEN

- STATISTIK

\begin{tabular}{|c|c|c|c|c|c|c|c|c|c|c|c|c|c|c|c|c|}
\hline Parameter & $\begin{array}{l}\text { Ver- } \\
\text { fahren }\end{array}$ & Konstante & $\begin{array}{l}\text { Log } \\
\mathrm{C}_{\text {org }} \\
\%\end{array}$ & DOC & $\mathbf{C}_{\text {hwl }}$ & $\begin{array}{l}\log N_{t} \\
\mathrm{mg} / \mathrm{kg}\end{array}$ & $\begin{array}{l}\text { Log } \\
\text { Sulfid }\end{array}$ & $\begin{array}{l}\text { Log } \\
F e_{\text {lös }}\end{array}$ & $\begin{array}{l}\mathrm{pH} \\
\left(\mathrm{CaCl}_{2}\right)\end{array}$ & $\mathrm{CaCO}_{3}$ & Sand & $\begin{array}{l}\text { Log } \\
\text { Ton } \\
\%\end{array}$ & $\begin{array}{l}\text { Log } \\
\text { Schluff }\end{array}$ & $\mathbf{R}^{2}$ & $\mathbf{P}$ & $\mathbf{N}$ \\
\hline $\log \left[R_{\mathrm{SO} 4(\mathrm{~L})}\right]$ & $S$ & $-1,23$ & & & & & 0,39 & & & & & & & 0,36 & $1,2 \mathrm{E}-08$ & 73 \\
\hline $\log \left[\mathrm{R}_{\mathrm{SO} 4(\mathrm{~L})}\right]$ & D & $-2,13$ & 0,01 & & & $-0,08$ & 0,42 & & 0,06 & & & 0,26 & & 0,34 & 7,4E-06 & 73 \\
\hline $\mathrm{M}_{\mathrm{SO} 4(\mathrm{~L})} 500 \mathrm{~d}$ & S & -466 & 261 & & & -227 & 166 & & & $-16,9$ & & & & 0,60 & $1,2 \mathrm{E}-13$ & 73 \\
\hline $\mathrm{M}_{\mathrm{SO} 4(\mathrm{~L})} 500 \mathrm{~d}$ & D & -351 & 254 & & & -218 & 165 & & $-3,19$ & $-16,5$ & $-0,43$ & & & 0,58 & 1,3E-11 & 73 \\
\hline $\mathrm{M}_{\mathrm{SO} 4(\mathrm{~L})}(\infty)$ & $S$ & -437 & 282 & & & -224 & 144 & & & $-19,8$ & & & & 0,58 & $5,6 \mathrm{E}-13$ & 73 \\
\hline $\mathrm{M}_{\mathrm{SO} 4(\mathrm{~L})}(\infty)$ & D & -337 & 237 & & & -172 & 138 & & $-6,33$ & $-18,0$ & 0,50 & $-3,70$ & & 0,56 & $4,4 \mathrm{E}-11$ & 73 \\
\hline $\mathrm{NI}_{\mathrm{SO} 4(\mathrm{~L})} 500 \mathrm{~d}$ & $S$ & 1,46 & & & & & & & & & & & $-1,24$ & 0,28 & 7,4E-07 & 73 \\
\hline $\mathrm{NI}_{\mathrm{SO} 4(\mathrm{~L})} 500 \mathrm{~d}$ & D & 0,81 & 0,20 & & & $-0,13$ & $-0,02$ & & 0,01 & & & 0,16 & $-0,99$ & 0,29 & $1,1 \mathrm{E}-04$ & 73 \\
\hline $\mathrm{VI}_{\mathrm{SO}(\mathrm{L})} 500 \mathrm{~d}$ & $S$ & 0,38 & & & & & & & & $-0,03$ & & & & 0,12 & 1,3E-03 & 73 \\
\hline $\mathrm{VI}_{\mathrm{SO} 4(\mathrm{~L})} 500 \mathrm{~d}$ & D & 0,14 & 0,64 & & & $-0,70$ & $-0,11$ & & $-0,04$ & $-0,01$ & & & & 0,19 & $3,2 \mathrm{E}-03$ & 73 \\
\hline $\mathrm{VI}_{\mathrm{SO} 4(\mathrm{~L})}(\infty)$ & $S$ & 0,99 & & & & & $-0,10$ & & $-0,08$ & & & & & 0,36 & 5,2E-08 & 73 \\
\hline $\mathrm{VI}_{\mathrm{SO} 4(\mathrm{~L})}(\infty)$ & D & 0,34 & 0,23 & & & $-0,26$ & $-0,10$ & & $-0,05$ & $-0,01$ & & 0,11 & & 0,37 & 3,1E-06 & 73 \\
\hline $\log \left[\mathrm{R}_{\mathrm{SO} 4(\mathrm{~S})}\right]$ & $\mathrm{R}$ & $-0,44$ & & $-0,01$ & & & & & & & & & & 0,05 & $1,0 \mathrm{E}-01$ & 35 \\
\hline $\log \left[\mathrm{R}_{\mathrm{SO} 4(\mathrm{~S})}\right]$ & D & $-1,63$ & $-0,50$ & $-0,02$ & & 0,79 & 0,04 & & 0,20 & & 0,02 & 0,44 & & 0,07 & $2,6 \mathrm{E}-01$ & 35 \\
\hline $\mathrm{M}_{\mathrm{SO} 4(\mathrm{~S})} 500 \mathrm{~d}$ & $S$ & 95,6 & & $-1,14$ & & & 11,2 & & & 175 & & $-127,82$ & $-75,57$ & 0,81 & $1,1 \mathrm{E}-10$ & 35 \\
\hline $\mathrm{M}_{\mathrm{SO} 4(\mathrm{~S})} 500 \mathrm{~d}$ & D & 42,4 & 10,0 & $-1,18$ & & $-22,4$ & 12,0 & & $-4,18$ & 194 & 0,26 & $-124,12$ & $-61,45$ & 0,80 & 2,7E-08 & 35 \\
\hline $\mathrm{M}_{\mathrm{SO} 4(\mathrm{~S})}(\infty)$ & $S$ & $-35,8$ & & & & & 3,50 & & 8,37 & & & & & 0,30 & 1,2E-03 & 35 \\
\hline$M_{\mathrm{SO} 4(\mathrm{~S})}(\infty)$ & D & $-18,8$ & $-2,32$ & & & $-1,49$ & 3,79 & & 1,29 & & 0,18 & $-19,01$ & & 0,27 & 1,8E-02 & 35 \\
\hline $\mathrm{NI}_{\mathrm{SO} 4(\mathrm{~S})} 500 \mathrm{~d}$ & $S$ & 0,33 & & & & & $-0,07$ & & & & & & $-0,21$ & 0,25 & 3,9E-03 & 35 \\
\hline $\mathrm{NI}_{\mathrm{SO} 4(\mathrm{~S})} 500 \mathrm{~d}$ & D & 1,32 & $-0,04$ & & & 0,15 & $-0,07$ & & $-0,03$ & & 0,00 & $-0,29$ & $-0,46$ & 0,21 & 5,7E-02 & 35 \\
\hline $\mathrm{VI}_{\mathrm{SO} 4(\mathrm{~S})} 500 \mathrm{~d}$ & $S$ & $-1,31$ & & $-0,01$ & & & 0,09 & & 0,33 & & & & & 0,66 & 5,8E-08 & 35 \\
\hline $\mathrm{VI}_{\mathrm{SO} 4(\mathrm{~S})} 500 \mathrm{~d}$ & D & $-1,16$ & $-0,15$ & $-0,01$ & & 0,01 & 0,09 & & 0,12 & & 0,01 & $-0,37$ & & 0,69 & $8,5 \mathrm{E}-07$ & 35 \\
\hline $\mathrm{VI}_{\mathrm{SO} 4(\mathrm{~S})}(\infty)$ & $S$ & 0,86 & & & & & 0,00 & & & & $-0,01$ & & & 0,28 & $7,2 \mathrm{E}-04$ & 35 \\
\hline $\mathrm{VI}_{\mathrm{SO} 4(\mathrm{~S})}(\infty)$ & D & 0,81 & $-0,09$ & & & 0,04 & 0,02 & & 0,00 & & $-0,01$ & $-0,02$ & & 0,22 & 4,1E-02 & 35 \\
\hline
\end{tabular}


Anhang 5 (Fortsetzung): Ergebnisse der linearen multiplen Regressionsanlyse zwischen unabhängigen und abhängigen Koeffizienten der Regressionsgeraden mit statistischen Kenngrößen.

KOEFFIZIENTEN

\begin{tabular}{|c|c|c|c|c|c|c|c|c|c|c|c|c|c|c|c|c|}
\hline Parameter & $\begin{array}{l}\text { Ver- } \\
\text { fahren }\end{array}$ & Konstante & $\begin{array}{l}\text { Log } \\
C_{\text {org }} \\
\%\end{array}$ & DOC & $\mathbf{C}_{\mathrm{hwl}}$ & $\begin{array}{l}\log N_{t} \\
\mathrm{mg} / \mathrm{kg}\end{array}$ & $\begin{array}{l}\text { Log } \\
\text { Sulfid }\end{array}$ & $\begin{array}{l}\text { Log } \\
F_{\text {lös }}\end{array}$ & $\begin{array}{l}\mathrm{pH} \\
\left(\mathrm{CaCl}_{2}\right)\end{array}$ & $\mathrm{CaCO}_{3}$ & Sand & $\begin{array}{r}\text { Log } \\
\text { Ton } \\
\%\end{array}$ & $\begin{array}{l}\text { Log } \\
\text { Schluff }\end{array}$ & $\mathbf{R}^{2}$ & $\mathbf{P}$ & $\mathbf{N}$ \\
\hline $\log \left[\mathrm{M}_{\mathrm{SO} 4(\mathrm{Alle})} \mathrm{A} 500 \mathrm{~d}\right]$ & $S$ & 0,12 & & & & & 0,50 & 0,14 & & & & & & 0,23 & $4,5 \mathrm{E}-06$ & 88 \\
\hline $\log \left[\mathrm{M}_{\mathrm{SO} 4 \text { (Alle) }} \mathrm{A} 500 \mathrm{~d}\right]$ & D & $-0,95$ & 1,88 & & & $-1,37$ & 0,53 & 0,10 & 0,11 & & $-0,01$ & $-0,86$ & & 0,33 & 1,5E-06 & 88 \\
\hline $\mathrm{VI}_{\mathrm{SO} \text { (Alle) }} \mathrm{A} 500 \mathrm{~d}$ & $S$ & 0,37 & & & & & 0,07 & & & & & & $-0,23$ & 0,10 & $3,4 \mathrm{E}-03$ & 88 \\
\hline $\mathrm{VI}_{\mathrm{SO} 4(\mathrm{Alle})} \mathrm{A} 500 \mathrm{~d}$ & D & 0,23 & 0,04 & & & $-0,03$ & 0,06 & & 0,00 & & & 0,04 & $-0,19$ & 0,05 & $1,2 \mathrm{E}-01$ & 88 \\
\hline $\log \left[\mathrm{M}_{\mathrm{SO} 4(\mathrm{~L})} \mathrm{A} 500 \mathrm{~d}\right]$ & $S$ & $-4,48$ & 5,45 & & & $-4,29$ & 0,00 & & & 0,28 & & & & 0,69 & $5,6 \mathrm{E}-13$ & 53 \\
\hline $\log \left[\mathrm{M}_{\mathrm{SO} 4(\mathrm{~L})} \mathrm{A} 500 \mathrm{~d}\right]$ & D & $-5,55$ & 5,31 & & & $-4,55$ & 0,42 & & & 0,19 & $-0,01$ & 0,20 & & 0,69 & $5,1 \mathrm{E}-11$ & 53 \\
\hline $\mathrm{VI}_{\mathrm{SO} 4(\mathrm{~L})} \mathrm{A} 500 \mathrm{~d}$ & $S$ & 2,39 & 0,18 & & & & 0,00 & & & & $-0,01$ & & $-1,86$ & 0,32 & 5,7E-05 & 53 \\
\hline $\mathrm{VI}_{\mathrm{SO} 4(\mathrm{~L})} \mathrm{A} 500 \mathrm{~d}$ & D & 4,97 & 0,56 & & & $-0,42$ & 0,01 & & 0,04 & & $-0,02$ & $-0,59$ & $-4,01$ & 0,37 & 1,4E-04 & 53 \\
\hline $\log \left[M_{\text {SO4(S) }} A 500 d\right]$ & $S$ & 0,58 & 0,00 & $-0,01$ & & 0,13 & 0,11 & & 0,20 & & & & $-0,26$ & 0,84 & $1,5 \mathrm{E}-11$ & 35 \\
\hline $\log \left[\mathrm{M}_{\mathrm{SO} 4(\mathrm{~S})} \mathrm{A} 500 \mathrm{~d}\right]$ & D & 0,87 & 0,08 & $-0,01$ & & 0,06 & 0,11 & & 0,20 & & 0,00 & $-0,12$ & $-0,35$ & 0,82 & 2,0E-09 & 35 \\
\hline $\mathrm{VI}_{\mathrm{SO} 4(\mathrm{~S})} \mathrm{A} 500 \mathrm{~d}$ & $S$ & $-0,48$ & & $-0,01$ & & & 0,09 & & 0,17 & & & & & 0,38 & $4,2 E-04$ & 35 \\
\hline $\mathrm{VI}_{\mathrm{SO} 4(\mathrm{~S})} \mathrm{A} 500 \mathrm{~d}$ & D & $-1,31$ & $-0,04$ & $-0,01$ & & & 0,08 & & 0,19 & 0,47 & 0,01 & $-0,03$ & & 0,42 & 1,9E-03 & 35 \\
\hline $\mathrm{M}_{\mathrm{NO}(\mathrm{Alle})} \mathrm{H} 50 \mathrm{Od}$ & $S$ & 247 & & & & & & & & & $-1,78$ & & & 0,51 & $3,2 \mathrm{E}-17$ & 108 \\
\hline $\mathrm{M}_{\mathrm{NO}(\text { Alle) }} \mathrm{H} 50 \mathrm{Od}$ & D & 202 & $-13,2$ & & & -58 & -13 & & 19 & & $-2,58$ & $-67,7$ & & 0,52 & $2,2 E-15$ & 108 \\
\hline $\mathrm{M}_{\mathrm{H} / \mathrm{Ges} \text { (Alle) }} 500 \mathrm{~d}$ & $S$ & 1,18 & 0,19 & & & & $-0,19$ & 0,04 & & & & & & 0,34 & 2,0E-09 & 108 \\
\hline $\mathrm{M}_{\mathrm{H} / \mathrm{Ges} \text { (Alle) }} 500 \mathrm{~d}$ & D & 1,03 & 0,47 & & & $-0,29$ & $-0,18$ & 0,04 & 0,02 & & $-0,01$ & $-0,17$ & & 0,35 & 9,9E-09 & 108 \\
\hline $\mathrm{VI}_{\mathrm{NO}(\mathrm{Alle})} \mathrm{H} 500 \mathrm{~d}$ & $S$ & $-3,33$ & & & & & & & 0,22 & & & & & 0,09 & $9,1 \mathrm{E}-04$ & 108 \\
\hline $\mathrm{VI}_{\mathrm{NO} 3(\mathrm{Alle})} \mathrm{H} 50 \mathrm{~d}$ & D & $-1,62$ & 0,54 & & & $-0,55$ & $-0,12$ & & 0,12 & & $-0,02$ & $-0,73$ & & 0,13 & 1,9E-03 & 108 \\
\hline $\mathrm{M}_{\mathrm{NO}(\mathrm{L})} \mathrm{H} 500 \mathrm{~d}$ & $S$ & 490 & & & & 202 & & & & & $-3,20$ & & & 0,40 & 5,6E-09 & 73 \\
\hline $\mathrm{M}_{\mathrm{NO}(\mathrm{L})} \mathrm{H} 500 \mathrm{~d}$ & $\mathrm{D}$ & 36 & $-18,6$ & & & 23,0 & -19 & & 33 & & $-2,33$ & 57 & & 0,42 & $1,5 \mathrm{E}-07$ & 73 \\
\hline $\mathrm{M}_{\mathrm{H} / \mathrm{Ges}(\mathrm{L})} 500 \mathrm{~d}$ & $S$ & 0,67 & 0,24 & & & & $-0,21$ & & 0,08 & & & & & 0,45 & 4,9E-09 & 73 \\
\hline$M_{H / \text { Ges.(L) }} 500 d$ & $\mathrm{D}$ & 0,84 & 0,31 & & & $-0,08$ & $-0,21$ & & 0,07 & & $-0,01$ & $-0,12$ & & 0,43 & $5,8 E-08$ & 73 \\
\hline $\log \left[\mathrm{VI} \mathrm{NO}_{(\mathrm{L})} \mathrm{H} 500 \mathrm{~d}\right]$ & $S$ & $-4,31$ & & & & & & & 0,37 & & & & & 0,27 & $1,4 \mathrm{E}-06$ & 73 \\
\hline $\log \left[\mathrm{VI} \mathrm{NO}_{(\mathrm{L})} \mathrm{H} 500 \mathrm{~d}\right]$ & $\mathrm{D}$ & $-3,18$ & 0,45 & & & $-0,66$ & $-0,01$ & & 0,23 & & $-0,02$ & $-0,46$ & & 0,28 & 9,9E-05 & 73 \\
\hline $\mathrm{M}_{\mathrm{NO} 3(\mathrm{~S})} \mathrm{H} 500 \mathrm{~d}$ & S & 107 & 76,0 & $-1,24$ & & & & & & & & & & 0,70 & 2,0E-09 & 35 \\
\hline
\end{tabular}


Anhang 5 (Fortsetzung): Ergebnisse der linearen multiplen Regressionsanlyse zwischen unabhängigen und abhängigen Koeffizienten der Regressionsgeraden mit statistischen Kenngrößen.

KOEFFIZIENTEN -STATISTIK -

\begin{tabular}{|c|c|c|c|c|c|c|c|c|c|c|c|c|c|c|c|c|}
\hline Parameter & $\begin{array}{l}\text { Ver- } \\
\text { fahren }\end{array}$ & Konstante & $\begin{array}{l}\log \\
\mathrm{C}_{\mathrm{org}} \\
\%\end{array}$ & DOC & $\mathbf{C}_{\mathrm{hwl}}$ & $\begin{array}{l}\log N_{t} \\
\mathrm{mg} / \mathrm{kg}\end{array}$ & $\begin{array}{l}\text { Log } \\
\text { Sulfid }\end{array}$ & $\begin{array}{l}\text { Log } \\
F_{\text {lös }} \\
\end{array}$ & $\begin{array}{l}\mathrm{pH} \\
\left(\mathrm{CaCl}_{2}\right)\end{array}$ & $\mathrm{CaCO}_{3}$ & Sand & $\begin{array}{l}\text { Log } \\
\text { Ton } \\
\%\end{array}$ & $\begin{array}{l}\text { Log } \\
\text { Schluff }\end{array}$ & $\mathbf{R}^{2}$ & $\mathbf{P}$ & $\mathbf{N}$ \\
\hline $\mathrm{M}_{\mathrm{NO} 3(\mathrm{~S})} \mathrm{H} 500 \mathrm{~d}$ & D & 155 & 73,0 & $-1,00$ & & 3,03 & $-0,56$ & & 1,62 & & $-0,63$ & 9,98 & & 0,68 & 1,2E-06 & 35 \\
\hline $\mathrm{M}_{\mathrm{H} / \mathrm{Ges}(\mathrm{S})} 500 \mathrm{~d}$ & $S$ & 2,28 & 0,49 & & & & $-0,12$ & & & & $-0,01$ & 0,46 & & 0,65 & $2,8 \mathrm{E}-07$ & 35 \\
\hline $\mathrm{M}_{\mathrm{H} / \mathrm{Ges} \text { (S) }} 500 \mathrm{~d}$ & D & 2,31 & 0,58 & & & $-0,13$ & $-0,12$ & & $-0,04$ & & $-0,02$ & 0,36 & & 0,63 & $3,9 \mathrm{E}-06$ & 35 \\
\hline $\mathrm{VI}_{\mathrm{NO} 3(\mathrm{~S})} \mathrm{H} 500 \mathrm{~d}$ & $S$ & 2,20 & 0,75 & & & & & & & & $-0,04$ & & & 0,21 & $9,4 \mathrm{E}-03$ & 35 \\
\hline $\mathrm{VI}_{\mathrm{NO} 3(\mathrm{~S})} \mathrm{H} 500 \mathrm{~d}$ & D & 2,82 & 0,28 & & & 0,44 & $-0,16$ & & $-0,20$ & & $-0,03$ & $-0,04$ & & 0,14 & $1,2 \mathrm{E}-01$ & 35 \\
\hline
\end{tabular}

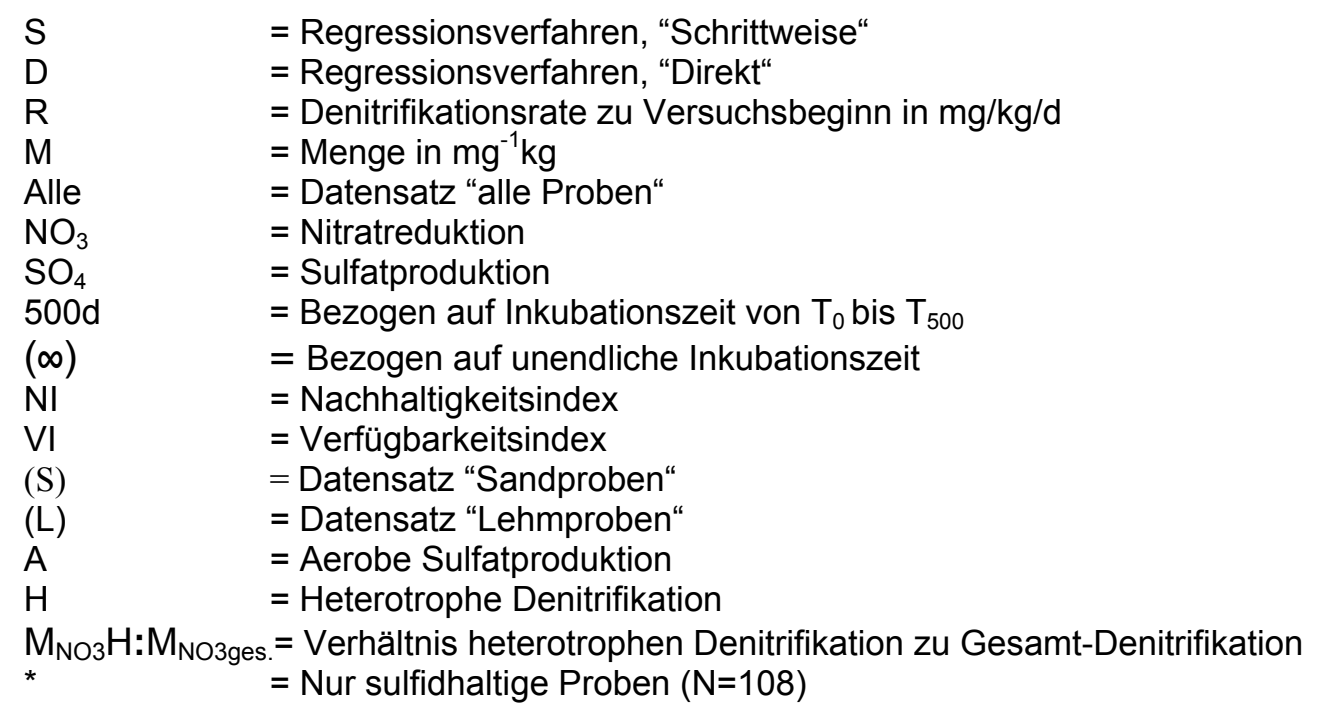




\section{Danksagung}

An dieser Stelle möchte ich allen Menschen, die mir bei der Anfertigung dieser Arbeit mit Rat, Tat und moralischer Unterstützung zur Seite gestanden haben, bedanken.

Ganz besonders möchte ich mich bei Herrn PD Dr. R. Well für die ständige Betreuung, zahlreiche Anregungen, Hilfestellungen und Diskussionen, die viel zum Fortschritt der Arbeit beigetragen haben, bedanken.

Herrn Prof. Dr. B. Meyer danke ich für die Überlassung des Themas und die Gestaltung der Lehre.

Herrn Dr. Chr. Ahl und Dr. K.W. Becker bin ich dankbar für die Unterstützung bei kleinen und großen Problemen.

Herrn Prof. Dr. N. Classen vom Institut für Agrikulturchemie möchte ich für die Übernahme des Koreferats danken.

Allen Mitarbeitern des Institutes für Bodenwissenschaften sei für ihre Hilfsbereitschaft und Mitwirkung an meiner Arbeit gedankt. Besonderes danken möchte ich Frau Susanne Richter, die mir bei den kraftraubenden Tiefbohrungen und bei den Laboranalysen half sowie für das Korrekturlesen. Bester Dank gilt auch für Frau Karin Schmidt, Frau Ingrid Ostermeyer und Frau Ulrike Hill für zahlreiche Laboranalysen.

Frau Angelika Reiz, Herrn Skrandies und Herrn Erwin Schiffczyk, Mitarbeitern des Institutes für Geochemie danke ich sehr für Ihre freundliche Hilfestellung, Arbeitsatmosphäre und Unterstützung bei der Schwefel-Analyse.

Für das Korrekturlesen bedanke ich mich sehr bei Herrn Ralf Bode.

Schließlich möchte ich Herrn Diop herzlich danken. Er hat durch seine entgegenkommendes Interesse, seine Bereitschaft und Geduld zum Gelingen der Arbeit beigetragen.

Für die Finanzielle Unterstützung bedanke ich mich sehr bei der DEUTSCHEN BUNDESSTIFTUNG UMWELT, ohne die die Durchführung der Arbeit nicht möglich gewesen wäre. 


\section{Lebenslauf}

Ozra Mehranfar

Geboren am 11.03.57 in Iran/Teheran

Schulausbildung

1963-1969 Schulbesuch in Teheran

1969-1975 Gymnasium in Bandaranzali/Iran

Hochschulstudium

1986-1987 Studienkolleg an der Universität Kassel/Witzenhausen

1987-1992 Studium der Internationalen Agrarwirtschaft an der Universität Kassel/Witzenhausen

1993-1995 Studium der Ökologische Umweltsicherung an der Universität Kassel/Witzenhausen

1998-1999 Wissenschaftliche Mitarbeiterin am Institut für

Bodenwissenschaften an der Georg-August Universität Göttingen

Promotion seit 01.07.1999 am Institut für Bodenwissenschaften der Universität Georg-August Göttingen 